The Methods of

Microscopical Research 


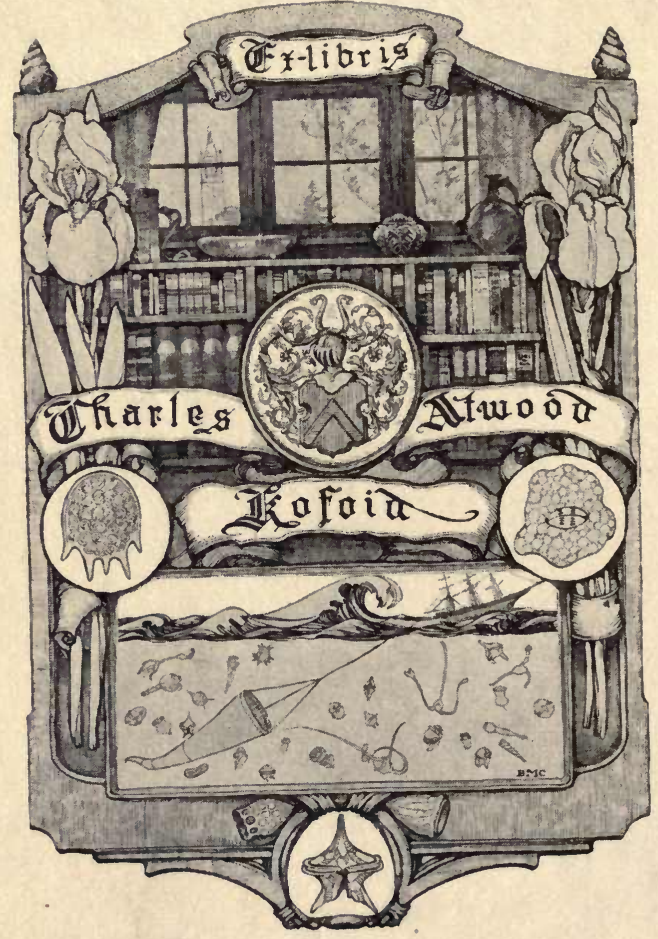




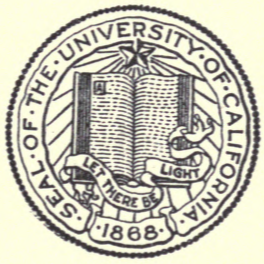

THE LIBRARY

$\mathrm{OF}$

THE UNIVERSITY

OF CALIFORNIA

PRESENTED BY

PROF. CHARLES A. KOFOID AND

MRS. PRUDENCE W. KOFOID 

THE METHODS OF MICROSCOPICAL RESEA RCH. 



\section{THE METHODS}

$\mathrm{OF}$

\section{MICROSCOPICAL RESEARCH}

A PRACTICAL GUIDE TO MICROSCOPICAL

MANIPULATION

SECOND EDITION

MUCH ENLARGED AND IN GREAT PART RE-WRITTEN

BY

ARTHUR C. COLE, F.R.M.S.

ALL RIGHTS RESERVED

Ionoon

BAILLIÈRE, TINDALL \& COX

20 \& 21, KING WILLIAM STREET, STRAND, W.C.

PARIS. 



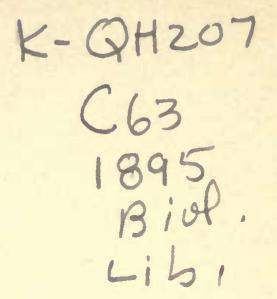

\section{PREFACE TO THE SECOND EDITION.}

"The Methods of Microscopical Research" formed a portion of the "Studies in Microscopical Science," a serial publication in four volumes, issued and edited by the author, and was embodied (as a treatise) with Volume II. of that work. The reception accorded to it induced the author to publish it as a separate work. The first edition was rapidly taken up, and the book soon became out of print and unobtainable. In response to a very general demand it is now re-issued, this (the second) edition being greatly enlarged and the work, indeed, almost entirely re-written. It embodies the full results of the author's thirty years' experience in microscopical work, all the formulæ from his private note book, all his own special processes and methods of working, and all his simple inventions for perfecting the work and ensuring absolute cleanliness and permanence in preparations. Nothing has been concealed, everything has been fully described and explained, and so plainly that the author entertains the hope that the treatise will be found really practical, and so will enable the student and the amateur microscopist to make preparations worth studying and retaining as cabinet specimens. 



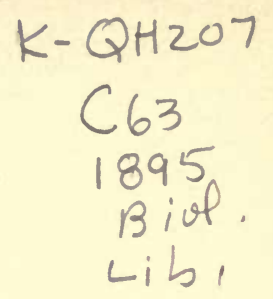

\section{PREFACE TO THE SECOND EDITION.}

"The Methods of Microscopical Research" formed a portion of the "Studies in Microscopical Science," a serial publication in four volumes, issued and edited by the author, and was embodied (as a treatise) with Volume II. of that work. The reception accorded to it induced the author to publish it as a separate work. The first edition was rapidly taken up, and the book soon became out of print and unobtainable. In response to a very general demand it is now re-issued, this (the second) edition being greatly enlarged and the work, indeed, almost entirely re-written. It embodies the full results of the author's thirty years' experience in microscopical work, all the formulæ from his private note book, all his own special processes and methods of working, and all his simple inventions for perfecting the work and ensuring absolute cleanliness and permanence in preparations. Nothing has been concealed, everything has been fully described and explained, and so plainly that the author entertains the hope that the treatise will be found really practical, and so will enable the student and the amateur microscopist to make preparations worth studying and retaining as cabinet specimens. 



\section{CONTENTS.}

INTRODUCTION.

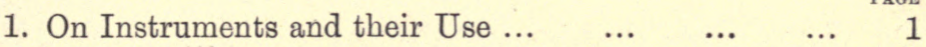

2. On Reagents, their Constitution and Action ... $\quad \ldots \quad 4$

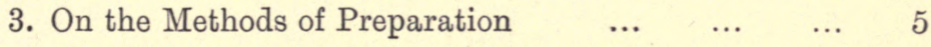

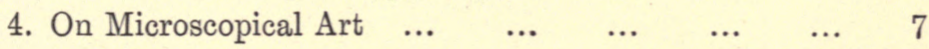

Chapter I.

$\begin{array}{lllllllll}\text { The Microscope } & \ldots & \ldots & \ldots & \ldots & \ldots & \ldots & 10\end{array}$

$\begin{array}{llllllll}\text { The Human Eye } & \ldots & \ldots & \ldots & \ldots & \ldots & \ldots & 13\end{array}$

Chapter II.

$\begin{array}{lllllll}\text { Instruments and their Use... } & \ldots & \ldots & \ldots & \ldots & 27\end{array}$

Chapter III.

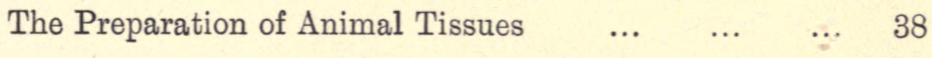

Chapter IV.

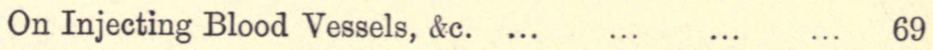

Chapter V.

$\begin{array}{lllllll}\text { On Staining Fluids and Staining } & \ldots & \ldots & \ldots & \ldots & 77\end{array}$

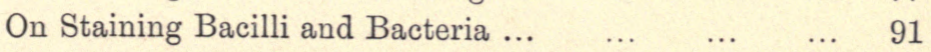


viii.

Contents.

$\begin{array}{llllllll}\text { Clearing Media } & \ldots & \ldots & \ldots & \ldots & \ldots & \ldots & 95\end{array}$

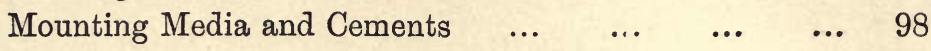

$\begin{array}{llllllll}\text { Embedding and Freezing } & \ldots & \ldots & \ldots & \ldots & \ldots & 109\end{array}$

On Section Cutting and Microtomes $\quad \ldots \quad \ldots \quad \ldots \quad \ldots \quad 119$

Chapter VI.

$\begin{array}{llllllll}\text { On Mounting } & \ldots & \ldots & \ldots & \ldots & \ldots & \ldots & 132\end{array}$

On the Preparation and Mounting of Insects $\quad \ldots \quad \ldots \quad 138$

On the Preparation and Mounting of Vegetable Sections 141

On the Preparation and Mounting of the Diatomaceæ ... 144

Chapter VII.

On Microscopical Drawing and Painting... $\quad \ldots \quad \ldots \quad \ldots \quad 161$

Chapter VIII.

$\begin{array}{llllllll}\text { On Photo-Micrography } & \ldots & \ldots & \ldots & \ldots & \ldots & 180\end{array}$ 


\section{THE METHODS \\ OF \\ MICROSCOPICAL RESEARCH.}

\section{INTRODUCTION.}

\section{I.-On Instruments and their Use.}

The investigation of minute structure, whether organic or inorganic, can be exhaustively and satisfactorily accomplished only by the assistance of adequate optical instruments. The organ of vision in man is so constructed that the distance of most distinct vision alternates between eight to ten inches, and the intermediates between these distances, from the normal eye of an adult. This is due to the alterable curvature of the crystalline lens which allows the practically parallel pencil of rays from a distant object, or the divergent rays from an object close to the eye, to be accurately focussed on the retina, or sensitive portion of that organ; but if the object is brought still closer to the eye than the normal distance of most distinct vision, it gradually 
loses its power of accommodation in exerting the strain required to render the crystalline lens sufficiently convex to focus the image of the object upon the retina; about two inches from the eye this action becomes impossible. Within the limits of accommodation megascopical characters only can be appreciated. If the eye were capable of altering the curvature of its crystalline lens indefinitely, its power of vision would become illimitable, and telescopes and microscopes and other such instruments would be found unnecessary. But the bounds of natural limitation can be conquered by artificial means, and the interposition of a sufficiently convex lens between the near object and the eye alters the direction of the rays of light which proceed from the object so as to bring them within the scope of natural vision, and herein lies the theory on which the microscope has been constructed. The nearer an object approaches the eye, the greater does its visual angle (or angle produced by the intersection of rays, or straight lines, from the extreme points of the object) become, and, consequently, a larger image is focussed upon the retina.

Optical instruments, therefore, are required on the very threshold of our "Studies" to enable us to enter the domains of histology and microscopical investigation, and they may be employed directly in a multitude of instances when the objects are of minute size. Equally numerous, however, are the substances and organisms which cannot be thus directly examined, but which require to be subjected to special processes and manipulation in order to render them suitable for microscopical examination. In the inorganic kingdom some 
minerals, rocks, and chemical substances, elementary and otherwise, are found in Nature in such minute particles as to render them suitable for immediate examination and study under the microscope, but, in the majority of instances, it is necessary either to pulverise, grind them down into the thinnest possible sections or sheets, or to precipitate them by chemical means ere they can be brought within the range of observation, and thus the petrologist must be provided with chisels, hammers, files and stones for grinding and polishing, and a variety of instruments adapted to the collection and subsequent treatment of specimens, whilst the chemist will require blow-pipes, test tubes, re-agents and balances, \&c., \&c. For investigations in the organic world, the vegetable histologist must provide himself with a vasculum, a trowel, dipping bottles, and all necessary collecting apparatus of all kinds, and with all the various instruments necessary to facilitate the examination of the unicellular and more delicate forms of plant life, whilst the more highly organised and complex examples will require dissection or sectionising and, in many cases, chemical treatment before they can be examined to any useful purpose. The animal histologist again will find his work inextricably connected with that of his brother botanist, and, to a large extent, will work hand in hand with him, but, as his observations and inquiries progress, there will arise necessities for a collection of tools including scalpels, scissors, bone forceps and saws, needles, forceps of different sizes and forms, section knives, as well as the all-important and indispensable microtome; whilst both 
students will require all the staining re-agents and mounting media necessary for the proper treatment and permanent preparation (as slides) of both vegetable and animal specimens, tissues and sections.

The study of both organic and inorganic histology is so complicated, that verbal descriptions, however exhaustive and perfect, are, without microscopical demonstration, in many cases, wholly inadequate; diagrams and drawings, therefore, are constantly employed to supplement the descriptions of the lecturer. In the preparation of such valuable aids to verbai description, the microscope is a most valuable, if not an indispensable adjunct, and it has become necessary, equally for the student, the investigator, the observer, the demonstrator and the lecturer, to thoroughly understand the construction and the use of the microscope itself, and of the various apparatus necessary for careful observations and investigations.

\section{II.-On $\mathrm{Re}=$ agents, their Constitution and Action.}

This is one of the most important branches of Microscopical Technology, and as staining is an art, so a thorough knowledge of the behaviour of the various tissues under the action of chemical re-agents is an absolute necessity alike for the observer and the student. It will be continually found that the optical means at the disposal of the histologist are insufficient or inadequate to the resolution of structure, and although much may be 
done with carefully directed and modified light and an equally careful arrangement of magnifying power, yet the utility of the judicious application of re-agents requires only to be understood to be appreciated. The knowledge of the chemical constitution, physical properties and modes of manufacture of re-agents, often leads to the discovery of their specific action on minute and ultimate structure, whether these may consist in a revelation of inherent qualities or in a modification of details. It thus becomes incumbent upon the histologist to most carefully scrutinise the action of every description of re-agent, and equally carefully and systematically to record the results of such examinations.

Re-agents may be used in a variety of ways for the elucidation of structure and the detection of the constitution of tissues and bodies. Some are employed as chemical tests, others as staining media to differentiate the tissues or to induce changes and appearances whereby certain properties are revealed, whilst others, again, are used as preservative media. To know exactly what to apply and how to apply it for the revelation of specific phenomena, and to obtain the best results is of the very essence of microscopical technique and manipulation.

\section{III.-On the Methods of Preparation.}

In order to arrive at a correct knowledge of, and as a means to their study, both organic and inorganic specimens and the great majority of organisms require preparation by special methods. 
Thus, the processes of pulverisation, levigation, sectionising, and grinding of bones, teeth, minerals, and rocks are beset by difficulties of detail which prove to be insurmountable obstacles to the tyro until he shall have acquired the necessary knowledge and skill, under which they to a great extent disappear, or are more or less easily surmounted. So, also, want of success in section-cutting, staining and mounting specimens is referable to the ignorance or neglect of minute precautions, to the want of absolute cleanliness in all processes, and the absence of the delicacy of manipulation without which success is impossible of attainment. In the opening pages of "How to Work with the Microscope," Dr. Beale makes the following observations:- "Manual dexterity, although subordinate to many higher mental qualifications, is as essential for the successful prosecution of microscopic observation as it is for that of every kind of experimental science. It assists us in the discovery of new means of inquiry and in devising methods by which difficulties may be surmounted. Without skilful manipulation we can neither teach by demonstration facts which have been already discovered, nor hope to extend the limits of observation and experimental knowledge. It is not, therefore, surprising that many of the most important facts which have been recently added to microscopical science, have been discovered by men who had previously well-trained themselves in experiment-particularly in practical chemistry and minute anatomical dissection. Improvements in

${ }^{1}$ Fifth edition, London, 1880, p. 1. 
the practical details of manipulation almost necessarily precede an advance in natural knowledge, and invariably promote and expedite true scientific progress."

But although manipulative skill is a very necessary adjunct to microscopical research, an attainment of the understanding of the general principles of action at the outset, sometimes proves to be the most arduous portion of the work, and very often is the only impediment to success. Practice and perseverance, brought to bear upon previously gained knowledge, are the only royal roads to manual dexterity, and it thus becomes the duty of the instructor to point out, not merely what path ought to be taken, but the various pit-falls which everywhere surround the beaten track, and how best to avoid them.

\section{IV.-On Microscopical Art.}

There are two ways in which microscopical objects can be drawn so as to become useful records of research. By the first of these, a rough diagrammatic representation may be made, without reference to accuracy of form or size; merely to display the observer's views concerning the structure of the object. The second method is to make an accurate drawing with due regard to the size and form of the object under the microscope. Both of these methods are valuable in themselves, but their usefulness becomes immeasurably enhanced when they are combined so as to afford scope to the artistic skill and scientific knowledge of the draughtsman. 
There is a great deal of truth in the statement that true art is the outcome of genius, but that does not in any way affect microscopical drawing; it will be found that patience and practice are sufficient to enable the student to overcome every obstacle, and to achieve the most satisfactory results in this department of art.

But whilst admitting the perfection to which many great artists in microscopical drawing have attained, and the great assistance to microscopical research afforded by the works of those who, in addition to correctness of vision and observation, possess an educated eye and a hand capable of drawing exactly what they see, give us the benefit of exquisite colouring in addition to correctness of outline and detail, who can sufficiently estimate the incalculable advantages bestowed upon microscopical science by micro-photography, which producing pictures absolutely faultless and free from such slight defects as are inseparable from drawings which, being dependent for their production upon human eyes, brain and nerves, must inevitably be liable to human imperfections, whilst a micro-photograph from the hands of a skilled observer and photographer is as inevitably free from all errors and defects, and provides for us an actual and perfect enlargement in all its features and detailscolour alone excepted-of the image focussed upon the plate? Micro-photography has indeed proved a boon to the microscopist, and the improvements which have been made and are daily being introduced in both apparatus and processes entitle us to believe that valuable and exquisite as are the pictures now produced by photography, the future 
has greater and even more wonderful results in store for us. It is indeed impossible to overrate the benefits conferred alike upon science, the student, the teacher and the lecturer by this invaluable art. 


\section{CHAPTER I.}

\section{On Instruments and their Use: The Microscope.}

The microscope, as an instrument of power in histological research, depends essentially in construction, upon its conformity with the laws of light and human vision. It has already been stated that an idea of the size of an object is arrived at through the size of the image focussed upon the retina, and that these dimensions vary in proportion as the object is brought near to, or removed farther from, the eye. When the object is brought close to the eye, its visual angle-that is, the angle formed by the crossing of the rays from the extreme points of the object, is larger than when it is placed farther off, and, consequently, the image on the retina is larger also. If this principle were capable of unlimited extension, it would obviously follow, that to continue to increase the magnification of an object, all one would have to do would be to bring the object closer and closer to the eye. But there is a limit to this natural power of microscopical vision in the human subject, and the eye fails signally to accomplish its office when the object is brought 
within about two inches from its surface. The reason of this is, that the crystalline lens of the eye, in assuming a more convex shape through the relaxation of the ciliary muscle, becomes overtaxed at this distance. If, now, a sufficiently convex lens be placed between the object and the eye, so as to enable the divergent rays to be accurately focussed upon the retina, the difficulty will be overcome, and, theoretically, microscopical vision would be illimitable. But, is it so? Most certainly not. The employment of artificial substances, such as crown and flint glass, diamonds, \&c., although they considerably extend the power of sight, do not do so ad infinitum. Here the limitation is purely material, as distinguished from the former instance, the human eye, which is defective not only materially, but physiologically.

The worker in the field of microscopical research need not, however, be appalled by these statements, for it will be found that the human organ of vision, in conjunction with the excellent appliances of modern invention, will enable him to approach, and sometimes even to solve satisfactorily, many of those philosophical problems which underlie the evolution of things, both animate and inanimate. In exemplification of this, a few instances may here be recorded.

The practical geologist who sallies forth into the field with lens in hand, may gather during his walk a variety of rocks, which, from their cosmological structure, point to an igneous origin; some of the specimens are coarse grained, whilst others defy the utmost scrutiny of the eye. The microscope is brought to bear upon the question, and he finds 
that a power of 500 diameters is generally the utmost degree of amplification he will require to employ; but that for all practical purposes powers of from 20 to 100 diameters suffice. With the assistance of the microscope he is enabled to pronounce with decision that the rocks are igneous; and more, from analytical and synthetical experiments he can show that certain coarse varieties, which are thoroughly crystalline (the crystals being simply held together by adhesive and cohesive forces, without the necessity of an interstitial binding substance), are of deep-seated origin, and consolidated under conditions of enormous pressure and length of time. He is, in like manner, able to affirm of the other varieties, what their mineral constituents are, or have been, and how they came to assume their present states. Thus he builds a part of the fabric of geological philosophy, and with what?-with a comparatively low power of the microscope.

To take an example from the organic world; the questions of the function of various parts of the body, are very often arrived at through a minute study of its members. The form and general appearance of the cells of glands such as the salivary glands, point to the functions they perform, whether they secrete or absorb, and how and when they perform their duties. The study of amœboid, and even of ciliary motion, under the microscope, does not require a power of magnification much beyond 700 diameters, whilst the life history of the minute forms of life known as germs (bacteria, \&c.), may be readily comprehended by the use of from 700 1,200 diameters. It is only when such things as the 



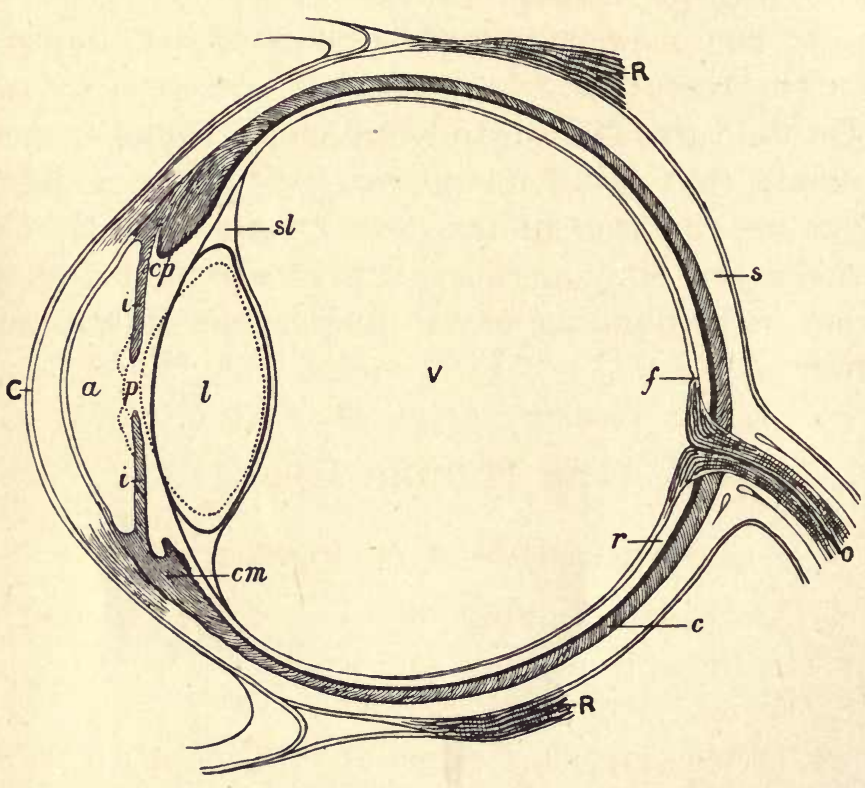

THE HUMAN EYE. 
delicate markings on the siliceous valves of Diatoms, or the artificial ruled lines on glass (Nobert's test) require to be made out, that powers beyond 1,200 diameters become useful; and it must be admitted that the scientific investigator does not lose anything, nor are any of his philosophical deductions vitiated by eschewing such powerful instruments, which, in the hands of the skilful, succeed in amplifying and resolving certain pretty structures; but, to the ordinary worker are, in reality, impediments to research.

On the threshold of this inquiry, it thus becomes evident, that an acquaintance with the general structure of the human eye, coupled with the principles of luminous energy, are necessary adjuncts to an understanding of the microscope, its use and power.

\section{The Human Eye.}

Description of Plate.

$a$, Anterior chamber of eye filled with the aqueous humour.

$c$, Cornea. $i$, iris. $p$, pupil.

$c m$, Ciliary muscle. $c p$, ciliary processes. $l$, lens.

$s$, Sclerotic coat. $c$, choroid. $r$, retina.

$f$, Fovea. $R R$, superior and inferior recti muscles.

$O$, Optic nerve entering the sclerotic and choroid coats.

$v$, Vitreous humour.

In all studies, whether of pure Microscopy as a Science, or whether of one of those departments of natural history in which the microscope is employed 
as an aid to vision, we must, at the outset, recognise the importance of a study of the human eye.

It may be the seat of many imperfections resulting from misuse, old age, or disease, which are apt to modify the conclusions we may draw from our observations, unless we are careful to study well into what lines such imperfections may lead us.

Nature has given us in this organ a means whereby all objects may be compared with each other, more especially as to size, colour, and general characters, and it must astonish the student, who thinks deeply, to find that so little is known definitely as to how we are able to appreciate magnitudes, colours, and forms. It is easy to say that the lenses of the eye focus a picture of the object upon the retina, and the irritations are carried by the optic nerve to the brain, but do we practically realise what this means?

Then, again, unless more of our senses than one are brought to bear upon a matter under consideration, we can scarcely form a true opinion upon our subject.

Take something which greets our vision for the first time. We know not what it is; we can see it, it is true, but we have to bring in the aid of other senses before we can arrive at a correct judgment; and even then, our judgment being the result of comparison, and also of experimental contact of substances with our senses-so to speak -opinions which are formed must, to a certain extent, be modified by the amount of other experience to which our nerve centres have been previously subjected.

Take two experts; give to each one a sphere 
composed of lead and tin. Upon asking them what substance they were handling they might probably guess, perhaps not; they would poise it in their hands, look at it, smell it, try to cut it, perhaps, examine its metallic lustre, and it would be very odd indeed if they could agree as to the composition of the alloy, unless settled by an assay upon the balance.

Has it ever occurred to the reader that such processes as these go on in Microscopy, and that it is necessary to carefully study the organ of vision in order to gain a true insight into the object presented to us? On reference to plate 1 , it will be seen that the eye is a nearly spherical ball, capable of many movements in its socket. It possesses an outer translucent covering called the sclerotic coat (or simply sclerotica) which may be seen at S. This is thick, horny, and opaque, except in its anterior portion.

This sclerotic coat envelopes about $\frac{5}{6}$ of the eyeball, and in common parlance is called the white of the eye.

The anterior transparent portion is called the cornea, and has the shape of a very convex watch glass. It is through this membrane that the light passes to the interior of the eye. The cornea and the interior portion of the sclerotica are covered with a mucous membrane.

Behind the cornea is a diaphragm of annular form called the iris; it is coloured and opaque, the circular aperture in its centre, $p$, being called the pupil.

The iris, $i$, serves the purpose of regulating the admission of light; it varies in colour in different 
individuals, and is the part referred to when we speak of the colour of a person's eye.

Behind the pupil is the crystalline lens, $l$, having a much greater convexity at its posterior surface than at the anterior.

The large posterior chamber is lined by the choroid coat, and this choroid has in front of it a delicate membrane called the retina.

The choroid coat consists of a highly vascular membrane containing pigment cells, filled with an intense black mucus, called the pigmentum nigrum.

The cavity behind the cornea is filled with a liquid called the aqueous humour, having a refractive index approaching that of $1 \cdot 3366$, while the larger cavity is filled with a transparent jelly, called the vitreous humour, possessing a refractive index of 1.3379, enclosed in a very thin, transparent sac, called the hyaloid membrane.

I have now described the principal apparatus of the eye, and may take some of the parts in detail.

The crystalline lens is built up of layers, increasing in density inwards, the effect of which is to diminish spherical aberration. This lens is enclosed in a transparent capsule, held in position by an elastic membrane. It can be changed in shape by means of a delicate muscular arrangement to adapt its focus for near or distant objects.

As glass lenses of varying curves have different focal lengths, so by altering the curves of the crystalline lens we are able to see objects distinctly which are situated in several focal planes.

The reader may have noticed that there is a near point at which objects can be seen most distinctly; this point varies in individuals, but averages from 
8 to 10 inches. As we move farther away from the object, although diminished in size, it may be seen more easily, and with less effort.

It would appear, then, that all objects are rendered apparently larger, as they continue to approach the eye, but a limit is soon found to this, as at a distance of six inches distinct and easy vision is not possible (except in very abnormal cases).

The reason of this is well-known--the anterior focal point of a convex lens when shortened lengthens the posterior conjugate focus, so that when an object is brought too near the eye the image of it is projected behind the retina, and the crystalline lens cannot accommodate itself to such extremes. But we know that objects can be seen distinctly at great distances apart, and it may be useful to demonstrate how this is brought about.

The real mechanism of accommodation has been much disputed, but the results, as observed, are, that the curvatures of the crystalline lens are altered as the observer adapts his eye to near or remote vision; increase of curvature, of course, shortening the focal length of the crystalline lens, and being better adapted for near vision, while the shallower curve is necessary for the distant view of remote objects. Helmholtz has shown that the radius of curvature of the anterior surface of the crystalline lens may be varied by means of the muscular arrangement, from 6 to 10 millimetres.

We may now cast another glance at the iris. This apparatus is really a continuation of the choroid tunic which lies between the sclerotica and the retina: it ends in front, in what are called 
ciliary processes. The small muscular ring surrounding the pupil is called the sphincter muscle.

Now, the principal use of the choroid tunic, or rather the pigmentum nigrum which it contains, is to absorb those rays of light which have passed through the transparent retina, preventing their reflection, which would interfere with the distinctness of the image.

By reference to the plate it will be seen that the choroid tunic, the retina, and sclerotica form the three outer rings, while the centre is ramified by nerve filaments and blood-vessels.

These nerve filaments and blood-vessels lie in the retina, which really forms a continuation and extension of the optic nerve; it touches the outer circumference of the iris at the front, and lies open as a cup-shaped disc in the interior of the eye; it receives the rays of light which have passed in turn through the cornea, aqueous humour, crystalline lens, and vitreous humour, and forms a picture at the focus of these.

The nerve fibres of the retina are excited probably by a product of the action of the light picture upon the visual purple, and the irritations are transmitted to the brain by the optic nerve, producing the sensation of vision.

The picture produced upon the retina has been compared with that produced by a photographic lens upon a screen or ground glass; but it will be seen that the instances are not strictly parallel.

In the eye the rays falling upon the cornea do not again encounter air, the picture is formed in the highly refractive substance, while in the photographic image air intervenes between the screen and the lens, and between the lenses themselves. 
Then, again, the adaptation of the eye to various distances is obtained by a process so dissimilar to that of the lens in the camera, that it is well no comparison should be instituted.

The retina has been previously described as a delicate membrane lining the choroid tunic, inside the sclerotica.

Now, if we make a section of the retina, and examine it under the microscope we shall find its structure to be as follows.

Starting from the junction of the retina with the vitreous humour, we have :-

(1) The layer of nerve fibres.

(2) The layer of nerve cells.

(3) The granular layer.

(4) The inner granular layer.

(5) The intermediate layer.

(6) The outer granular layer.

(7) A second fine membrane.

(8) The layer of rods and cones.

(9) Pigmentum nigrum of the choroid.

The retina is the terminal organ of vision, all the apparatus in front of it being merely for the purpose of securing that an accurate image shall be focussed upon it. As to how the luminous impressions yield to us such a definite idea of things is a question still under consideration; many have tried to solve it, but it is open to doubt whether we are any nearer the mark than those philosophers who lived 2,000 years ago.

There are several curious properties inherent in the retina. By means of the ophthalmoscope may be seen a point, a little out of the centre, where the optic nerve enters the eye. This spot is totally 
blind, it cannot perceive a trace of light, and if the image of an object falls upon this blind spot, that object is totally invisible. It is at this spot also where the blood-vessels enter the eye, and ramify through nearly the whole of the surface layers of the retina.

In the above description points only have been touched which directly bear on good or defective vision. On the other hand, enough has been advanced to show that this organ is liable to imperfections which may be, and are, extremely liable to modify all our observations made over the tube of the microscope.

Now, if we take an ordinary lens of glass and attempt to produce a picture with it, we find the centre alone is plainly visible-the lens is afflicted with what is termed spherical aberration, that is, the rays from its periphery are brought to a focus in a different plane to those occupying a central position.

But a small amount of spherical aberration is not readily detected by the student. It appears as a haze or fog of light over the object.

In the human eye this defect is not observable to any great degree, as the peripheral or more strongly refracting rays are cut off by the iris. Then, again, the curvature of the cornea is ellipsoidal rather than circular, so that the rays farthest from the axis are least deviated, while the two curves of the crystalline lens correct, so to speak, the one the other; and lastly, this lens is of such construction that its refractive power diminishes from the centre to the circumference.

Another defect in the eye is due to the different meridians having dissimilar degrees of curvature. 
If a set of concentric circles be observed with one eye, they are seldom all distinct at the same time, and there is produced a kind of Maltese-cross effect, not perceivable, perhaps, in many instances with large circles, but noticeable when drawn to such a size that the outer one is about two inches in diameter.

This defect is called astigmatism, and known to oculists as a common cause of headaches. Spasm of the focussing apparatus may derange the sphericity of the eye, and so affect vision. Strained vision is very subject to this. On the other hand, the same apparatus may be paralysed, and ordinary vision deficient, whilst the focussing of the microscope might possibly correct it.

Astigmatism has injuriously affected painters; Turner, for instance, whose later pictures are discovered to be slightly distorted, in consequence of the power of accommodation or self-correction having been lost through age.

In microscopic drawing, as with the camera lucida, the perspective may be misrepresented, in consequence of astigmatism, and thus endless disputes may arise even among the most careful observers.

We have now to deal with errors of refrangibility, and it will probably have been assumed that the eye apparatus is entirely corrected for colour. This is not the case, however, except when an object is in exact focus, and the reason that the error due to refrangibility remains practically unnoticed is that the distance between the focal point of the red and violet rays is extremely small. The error due to refrangibility may be noticed by means of the con- 
centric circles already referred to; by bright daylight adjust the eyes to some object twelve inches away, and without moving the eye insert at a distance of four inches a card inscribed with black circles, when a yellow and blue colouring will be plainly discerned.

In order that the reader may thoroughly understand the error of refrangibility, the picture afforded by the passage of a solar ray through a prism of glass may be thrown upon a screen; the rays are deflected unequally, the red least and the violet most.

It may be advisable here to state that the degree of dispersion of the rays of white light depends upon the medium through which the ray passes, and this amount of dispersion is measured by the distance of the most prominent dark lines in the spectrum from each other. The diamond disperses much less than crown glass, while the deflection of the ray is greater; but this is a subject beyond the scope of the present essay.

Now, beside these errors, there are others to which the microscopist should devote special attention; they are caused by small opaque particles existing in the transparent media of the eye-ball. These cast their shadow on the retina, and produce images which appear to exist outside the eye. These extra-retinal images often appear as globules, bacteroid-shaped bodies, or strings of minute pearls, and may be studied by directing the eye to a sheet of strongly illuminated opal glass, through a small aperture made with a fine needle in a piece of thin blackened cardboard.

When the microscope is used in a vertical position, 
these globules often gravitate to the centre of the cornea, and even after prolonged use of the inclined tube an observer may often be perplexed by the layer of mucus, or a lachrymal discharge covering the surface of the cornea.

Colour is a special sensation excited in the retina by rays of a definite wave length, and the reason why certain objects are presented to our view with colour is that when white light falls upon a given surface, some is absorbed, the remainder being reflected. If the green rays are reflected, then the object appears green, and if the red rays are alone reflected, then the object will be red.

The generally accepted theory of colour perception is based on the assumption that three kinds of nerve fibres exist in the retina, the excitation of which produces sensations of red, green and violet, and that modifications of these three sensations yield all intermediate tints.

This theory will explain some of the phenomena of colour blindness; if the nerve fibres which give their special sensation are paralysed, or are absent, the sensation only of the complementary tint will be transmitted with all the defects of the eye. It must not be forgotten that many phenomena consist more in errors of judgment than in absolute error of form or sensation.

Now in regard to errors of judgment, we must admit that all our estimations are made by comparison. In magnitude we are guided by the size of the retinal image as determined by the visual angle -for position we must have some starting point; and as for distance, every one knows how delusive an inexperienced estimate of this is. At'sea, a 
landsman could not judge of the distance of a passing vessel to a few miles, nor could we form any accurate idea of the size of any object emitting practically parallel rays unless we had something to compare it with.

We now come to a point which has been much disputed in the study of microscopy-binocular vision.

The two eyes move together as a system, so that we direct the two lines of regard to the same point in space and consequently see but a single image; but it is possible to see two-if one eye be displaced a little with the finger two images are seen, while if the other be displaced to a corresponding degree the one image is restored.

The value of binocular vision may be easily ascertained by experiment. When a picture is presented to the retina of each eye, the compound picture is much brighter than when one retina only is employed.

To each point of the retina of one eye there is a corresponding point in the retina of the other, and impressions produced on one of these points are in ordinary circumstances indistinguishable from similar impressions produced on the other.

When both retinæ are similarly impressed, the general effect is that the impressions are more intense than when one eye only is employed; and we also get a perception of relief, that is of form in its three dimensions.

Take two A eyepieces and look through them to the sky, so that two distinct circles are seen; now bring them together so that one circle overlaps the other, when this overlapping bi-convex portion will 
be found double the brightness of the remaining portions of the circles.

We are indebted to stereoscopic vision for the perception of relief or form in three dimensions, which occurs when the images falling upon the corresponding points of the two retinæ are not exactly similar. In looking at an object with both eyes the rays do not run parallel from one side of the object to the eye on that side, but the right eye centres itself to the left side of the object and viceversa. This may readily be seen by holding up a finger between our eyes and the wall, and looking at the latter. Two fingers may be seen projected on the wall, one of these is seen by the right eye and the other by the left; but our visual impressions do not inform us which picture is formed by either eye in particular. Now, while steadfastly looking at the wall, close the right eye and the left finger will disappear, while on shutting the left eye, the right finger is rendered invisible.

When two similar pictures are presented to the eyes, the impression is more vigorous and looked at with greater ease than when one eye only is employed; vision in this case is called pseudoscopic.

Binocular vision should be employed wherever practicable; it will be found much less trying to the eyes than monocular efforts.

It has now been shown that the human eye is extremely liable to imperfections, and being so, strict attention to details is demanded from the microscopist. Now, although the human eye is such a wonderful instrument, there are many problems it is unable to solve without extraneous assistance. Take, for example, the bunt of wheat, Tilletia faries. With 
the unaided eye, you will be able to discern nothing more than a black dust, the various details having to be made out by other means. Then again, with objects so minute as the diatom, Amphipleura pellucida, the object itself is almost invisible to the unassisted eye, to say nothing of the beautiful carvings with which the valves are embellished, and which exact for their elucidation the most perfect lenses with which we are acquainted, and the most accurate manipulation of the illumination. You may, indeed, see the contour of many forms of diatoms without extra optical assistance than that afforded us by nature, but not much more than this, as if the eye is approached too closely the picture falls behind the retina and is lost.

I have already mentioned the fact that starting with the distance of most distinct vision, continued approach to the eye finally renders the object invisible, the rays being thrown behind the retina, the mechanism of accommodation being insufficient to produce a curve deep enough to bring the picture to a short conjugate focus.

This can, however, be done by interposing a lens, or lenses, between the object and the cornea, so that a virtual image of the object is seen. These lenses form either a simple, or a compound microscope. 


\section{CHAPTER II.}

Is continuation of the subject of "Instruments and their Use," the author, holding in the highest estimation that invaluable treatise, Dr. Lionel S. Beale's "How to Work with the Microscope," as a thoroughly practical work, takes the liberty of quoting therefrom the following wise observations, which he considers cannot be too widely disseminated. "By describing the results of the investigations of others, a teacher may spread knowledge. By prosecuting original enquiries himself, he may contribute his mite to the gradually increasing stock of information; but by demonstrating to his pupils the successive steps by which conclusions in scientific enquiries have been at length arrived at, and by describing minutely the methods which have been actually employed in investigation, the teacher not only encourages his pupils to become original observers, and to investigate for themselves, but he may succeed in placing them in a position to commence their researches at the point where an enquiry has been abandoned by preceding observers.

"The opinion that it is only necessary to place an object in the field of the microscope in order to make out its structure, seems far too prevalent. 
Much of the disappointment suffered by many who are provided with microscopes may be traced to this erroneous idea. Too many look upon the microscope as a mere toy, and microscopical observation as an amusement, by the help of which time may be made to pass away pleasantly. Few are aware of the real interest derived from intelligent investigation, and the instruction afforded, and the facts for contemplation and thought easily to be obtained if only the observer will acquire the necessary dexterity and elementary knowledge to enable him to study with success. Many who have become interested in what was at first but rough and superficial investigation, have persevered and have at length become excellent observers, who have added new facts to our knowledge, or have rendered more accurate information which was already possessed."

It is not intended in this work to give minute descriptions of, or to compare one with another the microscopes and various apparatus supplied by the accomplished opticians in this and other countries, the details of which are fully given, and the instruments and accessories exhaustively described in so many works upon the microscope. The aim of the present treatise is rather to give thoroughly practical directions for the use of the instruments and apparatus, so that "research" and manipulation may go hand in hand.

For practical worl (as distinguished from delicate observations with the higher powers) the simpler and smaller-in other words, the "handier"-the microscope which is in constant use the better, and the lower the power that may safely be used to 
accomplish the work, also, the better. The use of the higher powers, and the more delicate accessory apparatus of the microscope follows when the preparations are made, and examination and observation commence. As a matter of course, when investigations have to be made during the progress of the work, powers sufficiently high must be used, but let it always be remembered that there is an axiom, "all the best microscopical work has been done with low powers" which, in reference to the aims of this treatise, means all the best and most perfect permanent preparations have been made with low powers, and are afterwards to be studied and examined under the higher powers and conditions of light, \&c., necessary to their elucidation and use as valuable aids to scientific instruction. For many purposes-such as the dissection of the larger insects, or the teasing out of the coarser tissues, a good "simple" microscope will be found extremely useful; but, as a rule, it is better to do almost everything under the compound microscope, and to patiently acquire the necessary skill which enables one to use botb ordinary and dissecting needles, small brushes, bristles, tubes, \&c., in the field of the microscope whilst using it; above all things it is important that, if possible, a binocular microscope should be used when many hours are spent in microscopical work, for not only is it natural, and therefore better, to use both eyes, but fatigue to the eyes is thus avoided, whilst it is certain, in respect of those who work with one eye only (unless the eyes are used alternately and often changed) that one eye becomes educated at the expense of the other, and to such an extent, that 
when the monocular instrument is exchanged for the binocular it is often found that the eyes have ceased to be a pair, and do not see alike.

Two eyepieces should be available with the working microscope, the ordinary, or $\mathrm{A}$, and a thoroughly good $\mathrm{C}$ eyepiece, and two objectives. After thirty years of constant work the author advises that these two powers should be a first rate $1 \frac{1}{2}$ and a $\frac{1}{4}$ inch. These, with the two eyepieces, will give a series of four magnifications and prove amply sufficient for all ordinary work, and the objectives can be conveniently used with a double nose-piece, except when dissecting or other such work is being done under the microscope and the nose-piece shall be found to interfere with free manipulation. It is well to take care that the objectives are as nearly as possible of the same length, in order to avoid the risk of breaking the slide under the microscope-a catastrophe very likely to occur either from momentary forgetfulness of which power may be in use, or hurry in changing the objectives. There is no difficulty in obtaining a $1 \frac{1}{2}$ and $\frac{1}{4}$ inch objectives so arranged as to require but little alteration of the rackwork of the microscope to focus them alternately. Whilst a $1 \frac{1}{2}$ inch objective will be found the most useful of all glasses for ordinary work and for most purposes, it will often be found necessary in the course of the work to make examinations of its parts and progress by means of the $\frac{1}{4}$ inch. With English microscopes it is advisable, as a rule, to use English objectives, but admirable glasses of great penetrating power and definition by foreign makers can now be obtained at a much less cost than those of English manufacture. The object- 
glasses made by Zeiss, Reichart and others, can scarcely be surpassed.

Flatness of field, penetration, definition, and entire absence of colour are the essentials of a thoroughly good objective, and all objectives not possessing these qualities should be rejected.

For working powers objectives with the lower angles of aperture are to be preferred, as it is most desirable to secure as much room as possible for manipulation between the preparation and the objective. For subsequent examination and study, however, high power objectives with high angles of aperture are invaluable, and oil-immersion objectives especially so. A thoroughly good fine adjustment is all-important to a good working microscope. The stage of a microscope intended for work should be a simple table, either circular or square (preferably the former), so that the specimen may be rotated and all its parts easily got at; there should be no mechanical arrangements whatever upon it (unless these are removable), so that the hands may have free scope; and the stage should be of ample size, so that if necessary it may form a rest for the hands whilst working.

For exhaustive observations and delicate investigations and the study of permanent preparations, the elaborate microscopes and beautiful apparatus now obtainable are of the highest possible value and utility, but for ordinary work they are not only quite unnecessary but too complicated to admit of satisfactory work being done under them with any comfort or convenience, whilst their great costliness and delicacy, and their liability to injury from chemicals and anything like rough usage, render them totally unsuitable as "working" microscopes. 
The following list will prove useful to the student who desires to fit up his laboratory, or workroom, with the most useful and necessary apparatus and requirements for microscopical study, investigations, and the making of preparations.

A good simple microscope, which, however, may be dispensed with if the student decides to work entirely with the compound microscope as already advised.

A thoroughly good and firm microscope-preferably a binocular instrument (which can also be used as a monocular) — with large stage, as already described, coarse and fine adjustments, plane and convex mirrors, and diaphragm, and having a fitting under the stage to receive an achrornatic condenser of a simple and inexpensive kind, a spot Lens, and a Polariscope.

\section{A Bullseye Condenser.}

Two objectives, $1 \frac{1}{2}$ and $\frac{1}{4}$ inch with double nosepiece.

Two Eyepieces, "A" and "C."

A Camera lucida for drawing objects.

A Stage micrometer divided into 100th and 1000 th of an inch.

A good Paraffin Lamp with metal chimney, and blue glasses to fit into the chimney to moderate the light.

A retort Stand.

A Spirit Lamp.

A small Pestle and mortar of Glass or agate.

A best "Cathcart" Microtome arranged for both the embedding and Ether freezing processes.

Two Section Knives and a Plane-iron for cutting Sections. 
A set of Scalpels.

Two pairs of ordinary surgical scissors (small and large) and one curved pair.

Two pairs of forceps (small and large), and a specially curved pair for manipulating cover glasses.

Four "Bulldog" forceps for compressing vessels when injecting.

A pair of Bone forceps.

Two thick curved needles in handles for lifting Sections.

Needles, small and large, and handles for them from which they can be readily removed and replaced when it may be necessary to change them.

Needles with flattened points and cutting edges, in handles.

Two Sewing Machine needles-which will be found most useful for dissecting and for laying out Sections.

A Bone saw.

An injecting Syringe, complete with Cannulæ and Stopcocks.

A Hypodermic Syringe for delicate injections.

A writing diamond.

Two pieces of good cork backed with lead.

Two small glass dishes for dissecting under water.

Three flasks for boiling.

A set of Test Tubes and rack.

Short Tubes, with corks, for storing small Sections.

Half oz., $1 \mathrm{oz}$. and $2 \mathrm{oz}$. Bottles, with Corks, for storing Sections.

A Washing bottle.

Six Dropping bottles. 
Six Dipping Tubes.

Six Pipettes.

Three Glass rods for stirring.

Some small glass tubing.

Some small india-rubber tubing.

Six small saucers of white porcelain and six large saucers.

Six Watch glasses.

Three small Black vulcanite photographic trays. These will be found most useful for selecting sections, which have been kept in spirit, when floated upon water placed in the trays. The black background to the white sections renders their selection perfectly easy.

Two small and one large Porcelain Photographic Trays, the deeper the better - these are for soaking Sections, when stained with Logwood, in Tap water.

Two Glass funnels and a measuring glass for 500 and one for 10 c.c.

One gross of 3 in. by 1 in. Glass slips.

One oz. each of (mixed sizes) thin glass circles No. 1 and No. 2.

Three in. by 1 in. slips with ground out cells.

Pure Tin cells, $\frac{1}{2}$ in. and $\frac{3}{4}$ in. in diameter.

Noтe.-Glass slips, thin covers and all tools and apparatus required for mounting; all cements, media and staining fluids, \&c., can be obtained at Mr. C. Baker's, 244, High Holborn, London, W.C.

\section{List of Chemicals ReqUired.}

Distilled water-the purest obtainable.

Glacial acetic acid.

Ordinary (best) acetic acid. 
Hydrochloric acid.

Nitric

Sulphuric

Best Methylated Spirit.

Alcohol, B.P.

Absolute Alcohol.

Ammonia B.P. Fort.

Ammonium Tartrate.

Alum (powdered).

Ammonium Chromate.

Ammonium Bichromate.

Arsenious acid.

Formic

Picric

,

Osmic

Acetate of Potass.

Bichromate of Potass.

Benzol.

Borax (powdered).

Canada Balsam, ordinary and hardened.

Absolute Phenol (Carbolic acid).

Carbonate of Potass.

Ferrocyanide of Potass.

Carmine (best).

Celloidin (in Flakes or so-called "shavings").

Chloride of Gold (a 15 grain tube).

Chloroform.

Chromic acid.

Clove oil.

Collodion.

Ether · 720 B.P.

Gelatine ("Gold label").

Glycerin.

Gold size. 
Gum arabic.

Hæmatoxylin (Crystals).

Nitrate of Silver.

Potassium in Cylinders for making Liq. Potass.

Picrate of Ammonia.

Sodium Chloride.

Sulphate of Magnesia.

Simple Syrup.

Turpentine.

Xylol.

The following tables will be found very useful :-

FRENCH FLUID MEASURES.

The cubic centimètre, usually represented by "c.c." is the unit of the French measurement for liquids. It contains nearly seventeen minims of water; in reality, it contains 16.896 minims. The weight of this quantity of water is one gramme.

Hence it will be seen that the cubic centimètre and the gramme bear to each other the same relation as our drachm for solids and the drachm for fluids, or as the minim and the grain. The following table will prove to be sufficiently accurate for microscopical purposes:-

Cubic centimètres.

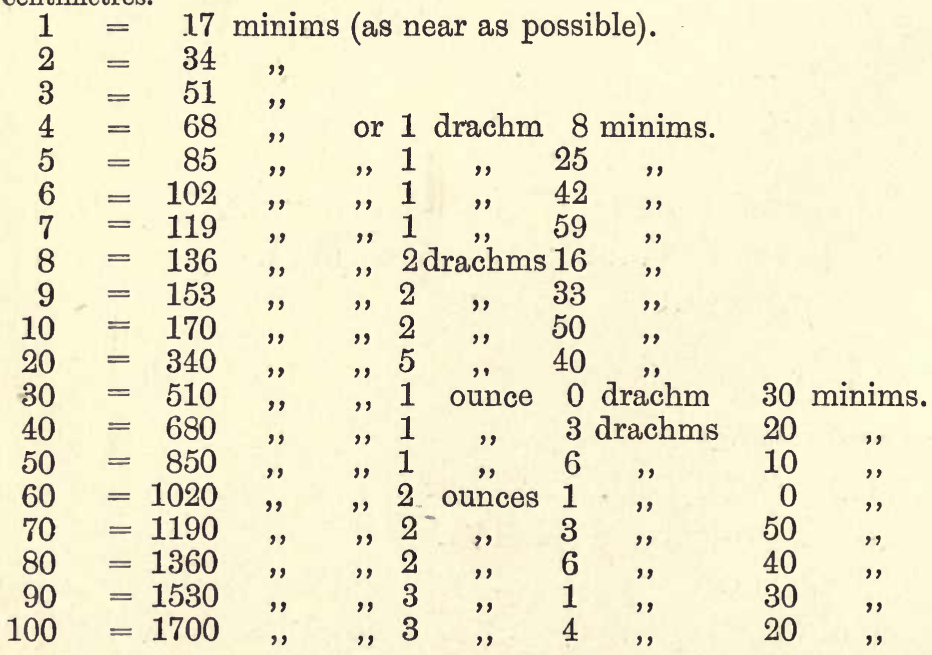




\section{THE CONVERSION OF FRENCH INTO ENGLISH WEIGHT.}

Although a gramme is equal to 15.4346 grains, the decimal is one which can never be used in microscopy; hence in the following table it is assumed to be $15 \frac{2}{5}$ grains, which is the nearest approach that can be made to practical accuracy :-

Grammes.

\begin{tabular}{|c|c|c|c|c|c|}
\hline 1 & $15 \frac{2}{5}$ & rains. & & & \\
\hline 2 & $30 \frac{4}{5}$ & ," & & & \\
\hline 3 & $46 \frac{1}{5}$ & , & & & \\
\hline 4 & $61 \frac{3}{5}$ & , & or 1 drachm & $1 \frac{3}{5}$ & grain. \\
\hline 5 & $77^{\circ}$ & ", &, $1 \quad$, & 17 & grains. \\
\hline 6 & $92 \frac{2}{5}$ & , &, 1 & $32 \frac{2}{5}$ & ," \\
\hline 7 & $107 \frac{\frac{4}{5}}{123^{\frac{1}{1}}}$ & ", & " 1 & $47 \frac{4}{5}$ & , \\
\hline 9 & $\begin{array}{l}123 \frac{1}{5} \\
138 \frac{3}{5}\end{array}$ & $"$ & "2 2 drachms & $\begin{array}{l}53 \frac{1}{5} \\
183\end{array}$ & , \\
\hline 10 & $154^{5}$ & ", & , 2 & $\begin{array}{l}18 \frac{5}{5} \\
34\end{array}$ & ", \\
\hline 11 & $169 \frac{2}{5}$ & ", &, 2 & $49 \frac{2}{5}$ & , \\
\hline 12 & $184 \frac{4}{5}$ & ", &, 3 & $4 \frac{4}{5}$ & , \\
\hline 13 & $200 \frac{1}{5}$ & , &, 3 & $20 \frac{1}{5}$ & ", \\
\hline 14 & $215 \frac{3}{5}$ & ," &,, 3 & $35_{\frac{3}{5}}^{3}$ & ", \\
\hline 15 & $231^{\circ}$ & ," &, 3 & $51^{\circ}$ & ", \\
\hline 16 & $246 \frac{2}{5}$ & ", & , 4 & $6 \frac{2}{5}$ & ", \\
\hline 17 & $261 \frac{4}{5}$ & ", & , 4 & $21 \frac{4}{5}$ & , \\
\hline 18 & $277 \frac{1}{5}$ & , & ,, 4 & $37 \frac{1}{5}$ & , \\
\hline 19 & $292 \frac{3}{5}$ & ," &, 4 & $52 \frac{3}{5}$ & ", \\
\hline 20 & $308^{\circ}$ & ," &, 15 & 8 & , \\
\hline 30 & 462 & ", &, 7 & 42 & , \\
\hline 40 & 616 & ", &, 10 & 16 & $"$ \\
\hline 50 & $=770$ & , &, 12 & 50 & ," \\
\hline 60 & $=924$ & $"$ &, 15 & 24 & , \\
\hline 70 & $=1078$ & $"$ & ,,17 & 58 & , \\
\hline & $\begin{array}{l}=1232 \\
=1386\end{array}$ & $"$ &, 20 & 32 & ", \\
\hline 10 & $=1540$ & ", & , 25 & $\begin{array}{r}0 \\
40\end{array}$ & ", \\
\hline
\end{tabular}




\section{CHAP'IER III.}

\section{The Preparation of Animal Tissues.}

THe majority of animal tissues require hardening to a greater or less extent, whilst some, on the contrary, must be softened before sections can be cut from them. The various processes for embedding and infiltrating organs and tissues have, however, recently been brought to such perfection that not only can much of the hardening once found necessary be dispensed with, much time saved and possible injury avoided, but-and this is a very important consideration-the risk of distortions and the obliteration of important features and properties in the tissues, which it is all important to their study should be carefully preserved, are reduced to a minimum or altogether avoided by the less heroic and more delicate processes now in vogue.

Hardening agents, in respect of the effects produced by them upon tissues, \&c., form two divisions: (1) those (e.g., alcohol and nitric, and picric acids, \&c.), which do not interfere with the subsequent processes for staining the tissues and sections; (2) those (e.g., osmic and chromic acids, \&c.), which do more or less affect the delicacy of the staining and the action of staining re-agents. 
In hardening tissues it is all important to secure that the specimens shall be thoroughly penetrated by the liquid in which they are placed, and to this end it is necessary to immerse them in an abundance of the liquid-say into 100 times their bulk, or even more-and to divide them into sufficiently small pieces to ensure this result, regard, of course, being had to the attainment of sections of sufficient size to show the structure and features of the various organs - this latter consideration, in these days of large sections, being obviously a matter of the greatest importance.

The tissues must be as fresh as possible. Parts which have to be treated by the gold or silver processes must be immersed within half an hour after death; or they will cease to be susceptible to the action of re-agents. The time of year also must be a matter of consideration, summer and hot weather being, of course, less favourable to the preservation of animal matter than the cold of winter. No tissue can be placed too early in the preservative medium; all useless matter, such as the contents of the stomach or intestines, must be carefully washed away with $\frac{3}{4}$ per cent. salt solution. As soon as a fluid has become "fouled" by the tissues placed in it, it should be changed; as a rule, frequent changes are advisable.

All tissues hardening in chromic acid solutions must be examined daily, after the first few days, to prevent them from becoming brittle and thus spoiled. Different tissues placed in the same medium should be separately examined and tested. Some may have become sufficiently hardened and require immediate removal, whilst a more prolonged 
immersion may be necessary for others. No tissue should be allowed to remain in chromium fluids until it is quite hard, if, as is commonly the case, it is necessary to complete the hardening in alcohol to which it should be removed when it has become tough, though still retaining its elasticity. When transferring a tissue to alcohol to complete its hardening, all trace of the medium in which it has already been immersed must be removed by prolonged soaking in cold water, which should be frequently changed during twelve or twenty-four hours, or until the last washings shall be colourless. Tissues thus prepared must not be put at once into full strength spirit, but first placed in methylated spirit and water ("half and half") for twenty-four hours, thence into pure methylated spirit. It is most important that the shrinkage of the tissues caused by the hardening processes should be uniform as well as gradual; it is therefore again obvious that the greatest care must be taken to use fluids which will penetrate the whole tissue or organ, lest the surfaces merely should become hardened whilst the interior portions remain soft and even become decayed.

As a rule it is advisable to commence with weaker, and to advance by degrees to stronger, solutions in order to ensure penetration. A book should be kept in which notes can be made of the nature of the fluids in which organs and tissues are hardening, the dates of changing or substituting fluids, \&c. Each bottle should bear a letter or number to correspond with the letter or number registered against it in the note book. Tissues and organs may be wrapped in pieces of linen, and a label bearing its 
number to correspond with the note book attached to each parcel. In this way many specimens can be hardened together in the same fluid, and much space and waste of fluid avoided without any risk of confusion or error. No hard-and-fast line can be drawn in respect of the time during which the various organs and tissues should remain in the hardening fluids; careful experiments and notes, judgment and experience are necessary in this as in all other chemical work. Again, many tissues require hardening by different processes according to the elements in their structure which it is desired to demonstrate. For instance, to display the corpuscles and nerves of the cornea it must be treated with gold, whilst in order to show the cell spaces the silver process must be resorted to. The special methods of hardening many of the various tissues and organs will therefore be found included under more than one process.

\section{Alcohol.}

When alcohol is employed as the sole hardening agent it should be used strong (i.e., of 95 per cent.), indeed it will be found necessary in some cases to use absolute alcohol. The specimens are to be immersed in an abundance of the spirit, which must be frequently changed during the first few days. It is advisable in many instances to suspend the specimens, either wrapped in linen or by a strong thread, in the alcohol. Some tissues will become sufficiently hardened in a few days, whilst others, and especially large specimens and whole organs, will require weeks. The more frequently the 
alcohol is changed at first, the more perfect, as well as the more rapid, will be the hardening.

\section{Nitric Acid}

should be used in an aqueous solution of from 5 per cent. to 15 per cent., and the specimens may remain in it from fourteen to twenty-one days. When it shall prove desirable to complete the hardening of a specimen in spirit it may be removed from the nitric acid and placed in the spirit after a much less prolonged immersion in the acid. Great care must be taken to remove from the specimen all trace of the acid by prolonged soaking in water, frequently changed, before placing it in alcohol.

\section{Chromic Acid.}

Make a 1 per cent. solution in water and reduce its strength to $\frac{1}{4}, \frac{1}{6}$, or such other percentage as may be required. Specimens must be immersed in large quantities of this solution, in which they may remain for days or even weeks, the length of time during which they should be subjected to its action being determined by the size and nature of the specimen.

Mucous tissues harden rapidly in this medium. Nervous tissues, spinal cord and brain require weeks. Bone from which the muscles (but not the periosteum) have been removed should be steeped in a very weak solution, commencing with a $\frac{1}{10}$ per cent. and increasing to $\frac{1}{2}$ per cent. with frequent changes during a period of ten days. It is then to be decalcified by immersion in chromic and nitric fluid until a needle can be passed through it. It is then 
to be washed free from all trace of the acids, and transferred to strong spirit. Teeth may be treated in the same manner.

In all cases a weak solution should be employed at first and strengthened each time it is changed. The specimens should be examined from time to time, and removed when they shall have acquired the proper condition. The greatest care must be exercised lest they become brittle. When removed from the chromic acid solution they should be soaked in water, frequently changed, for at least twenty-four hours, and then transferred, for keeping, to 95 per cent. alcohol.

Muscle.-A piece of muscle from an animal recently killed is steeped in a small quantity of a $\frac{1}{6}$ per cent: solution for a week. The cleavage of its sarcous substance is shown by teasing a small piece in glycerine.

Nerve.-Harden a piece of metacarpal nerve of a horse, or sciatic nerve of a smaller animal for ten days in a $\frac{1}{6}$ per cent. solution. Stain a bit in logwood, then tease it in glycerine thoroughly. This shows its connective tissue.

\section{Chromic Acid and Alcohol.}

Mix one part of a $\frac{1}{6}$ per cent. solution of chromic acid with two parts of methylated spirit. This should be done when required for use.

The following tissues may be placed in this, then transferred to spirit. The time required must be judged according to directions already given:-

The whole of one cornea, to show stratified epithelium; a piece of small intestine, to show non- 
striped muscle; heart and pericardium of a small animal; small arteries and capillaries from the brain of a sheep, after scraping away the brainsubstance; middle-sized artery-such as metacarpal of the horse; trachea and lungs. These are gently injected and then immersed in the fluid.

The lips, tongue, salivary glands, tonsils, œsophagus (distended and tied), stomach (after washing away its contents with $\frac{3}{4}$ per cent. salt solution), small and large intestines, liver in half-inch cubes, ureter and bladder (distended), ovary, fallopian tubes, uterus (distended per vaginam) may be placed in the solution.

Besides the above, which may be taken from either a dog, cat, or guinea pig, the following should be obtained:-The thymus gland of an infant; skin of scalp, finger and palm of hand, sole of foot from the human subject; also a nail. The eye of an ox divided transversely just behind the cornea for the ciliary muscle, sclerotic, cornea, and iris; also the choroid and retina. Of course both halves have to be used. The prostate gland and penis of a guinea pig. The cervix uteri of a cow. Mammary gland of an animal near the full period of gestation. The placenta of a cat, or guinea pig. The umbilical cord, which must be cut into pieces an inch long, and hardened for two days in Müller's fluid before being placed in the present medium.

All the above tissues must be daily examined after the third day, and each transferred to spirit after it has become tough. Moreover, the fluid should be changed after the first twenty-four hours, and at intervals of a few days afterwards. 
When many different tissues are in the same jar, the quantity of fluid must be such that the upper surface of the tissues extends half way up the entire fluid, that is to say, the stratum of tissues and stratum of clean fluid over them should be of equal depth.

\section{Bichromate of Potass.}

Make a 2 per cent. solution of bichromate of potass, with ordinary water.

Meso-rectum of Cat.-Pin this out on cork, and float it cork upwards on the solution for seven days.

Liver.-After injecting the portal vein with blue gelatine mass, and the hepatic artery with carmine gelatine mass, the liver may be hardened in the solution to toughness, and, of course, finished in alcohol.

Spinal Cord of Ox.-Pieces an inch long may be placed in the solution-frequently changed-for from three to five weeks.

Spinal Cord of Ox, Horse, or Sheep. If pieces about an eighth of an inch long be macerated two or three days in $\frac{1}{8}$ per cent. of the solution, the anterior horn of the spinal cord snipped out with scissors, and teased in carmine solution, then pressed with a cover glass, using Farrant's medium, they will show the isolated multipolar nerve cells very beautifully. The sympathetic nerve cells of the frog can be isolated in the same manner.

Cornea of cat, rabbit, or guinea-pig should also be hardened in a 2 per cent. solution for ten days. The lens in 1 per cent. solution for one week. 
Ovaries of the Cow in small pieces should be macerated in very dilute solution to isolate the large, branched, pigmented cells of the Corpora lutea.

\section{Ammonium Bichromate.}

A 2 per cent. solution made with ordinary water may be employed.

This solution is preferred by many to the potass salt solution. It is used in the same way. Columnar epithelium may be prepared as permanent specimens by placing a piece of fresh intestine of dog, cat, rabbit, \&c., in 1 per cent. solution for two days; then steeping an hour or two in water and scraping off the epithelium and staining. The cells have to be separated with a needle, and may be mounted in Farrant's medium, or in glycerine jelly.

\section{Chromate of Ammonium.}

A 5 per cent. solution is used.

If a newt's liver (in small pieces) and pieces of the small intestines be placed in the solution for forty-eight hours, the liver cells and columnar epithelium may be obtained, as in the case abovementioned. The goblet cells of Klein can be perfectly preserved in glycerine jelly in this way. The mesentery of the newt may be placed in the solution at the same time, and taken out after twenty-four hours. This shows the non-striated muscle fibre beautifully. The isolated gastric glands of a small mammal may be obtained in the same way by placing pieces of the fresh mucous membrane for three days in the solution. The 
testes of the newt should be placed in the solution for twenty-four hours, then cut into, and their contents squeezed out on to a slide. The spermatozoa are thus obtained as a permanent preparation.

Another important use of the above solution has been pointed out by Heidenhain. If small pieces of kidney be placed in the solution for forty-eight hours (the cortex should be chosen), the preparation shows the cells of the uriniferous tubules and their peculiarities as no other method, probably, will show them.

\section{Müller's Fluid.}

This is made by dissolving twenty-five grms. of potass. bichrom. and ten grms. sodæ sulph. in 1000 c.c. of water.

Müller's fluid has great penetrating power. It hardens slowly, taking, it may be, from five to seven weeks. It is useful as a commencing agent, to be followed by another of greater shrinking power, such as chromic acid and spirit solution, ordinary alcohol, \&c. Very even shrinkage may thus be obtained. For example, if the fresh nasal septum is cut out and placed for two days in Müller's fluid, then for a week in chromic acid and spirit solution, afterwards in weak, then in pure methylated-spirit, the olfactory epithelium will be obtained in excellent preservation.

After the above explanation no difficulty will be found with the following delicate structures, namely: -Developing tooth, the adenoid tissue of lymphatic gland, spleen, thyroid gland, supra-renal-capsules (of the horse, by preference), sympathetic ganglion, 
olfactory epithelium, cochlea, testis, epididymis and vas-deferens, ovary, human placenta, \&c.

\section{Müller's Fluid and Spirit.}

Take three parts of Müller's fluid and add to it one part of methylated spirit and keep it in a dark place. The mixture should be made only as required.

This is especially useful for the central nervous system. The removal of the brain and spinal cord without injury may here be described. Immediately after death the skin is removed, or at least the skin over the back and neck. Then separate the neck and head from the trunk about the middle of the neck. Next clear away the muscles on each side of the vertebral spines and clip away every spine as close as possible with scissors or bone forceps. Next with ordinary forceps grasp the laminæ, which cover over the spinal cord, one lamina at a time, and break it outwards by inserting one blade of the forceps within the neural canal, the other on the upper surface of the lamina. Now advance down the spine, breaking the laminæ outwards right and left till all the cord with its membranes is exposed. The head portion is dealt with in like manner by breaking off, bit by bit, the top of the skull, inserting one blade into the interior, of course, after clearing away the skin and muscles.

The spinal cord and membranes of a cat, dog, or rabbit should be carefully taken out and suspended in a deep, narrow vessel. The fluid should be changed at the end of twenty-four hours, then after a week. At the end of this time, divide into pieces 
an inch long, and continue the hardening for another week or two, as may be required. It may be replaced by a 2 per cent. solution of bichromate of ammonium for two weeks, and the pieces preserved in spirit, or by Hamilton's method (chloral hydrate, twelve grains, water, one ounce). The cerebellum, cerebrum, and, of course, the medulla oblongata may likewise be hardened. In the case of the two former, divide into suitable pieces to ensure perfect penetration.

The tendo achilles of the calf, and the metacarpal nerve of the horse, each in inch-long pieces; also the posterior half of the eye-ball of a pig (for the retina) may be hardened with advantage in this fluid.

\section{Absolute Alcohol.}

This should have a specific gravity of 0.795 . It hardens in twenty-four hours, with much shrinking. It is used for secretory glands, notably the pancreas, which must be placed direct in it, or the gland may spoil by partial self-digestion. The salivary glands, the lachrymal glands, pieces from both ends of the stomach for the gastric glands, the pancreas in pieces, \&c., should be placed in it at once.

If the lymphatic gland of a horse, ox, or smaller quadruped is injected with 2 per cent. Prussian blue fluid by Klein's method (introduce a glass pipette filled with the fluid into a lacteal near a mesenteric gland and blow the solution into it), and then placed in absolute alcohol, we have the lymph sinuses well injected. The muscle structure of the beetle or crab is shown well by placing a beetle or the ampu- 
tated limb of a crab for a week in absolute alcohol. A bit of muscle is scooped out, stained and mounted, after being teased with needles. Non-striped muscle may be demonstrated thus:-Kill a small animal and wash out a length of the, small intestine with salt solution, distend it with absolute alcohol, and tie both ends, then suspend it in the alcohol for twelve hours. With a pair of blunt-pointed forceps now tear off strips from the outer surface, which will include the longitudinal muscle structure; stain them and mount in balsam.

\section{Picric Acid.}

Make a cold saturated aqueous solution. Small pieces of tissue harden in this in from twelve to forty-eight hours. It is excellent for decalcifying fœtal bones which may be left in it, and tested from time to time with a needle, which ought to be pushed easily through them. No time can be specified, in some cases perhaps weeks may be required. Prepare the varieties of cartilage with this solution. The aorta (pieces) of a large animal (horse, or ox), also a cornea to show its fibrous tissue, the thymus gland of an infant, also a lymphatic gland may all be well prepared by remaining in this solution twenty-four, thirty-six, or perhaps forty-eight hours. The intracellular plexus of fibrils may be well shown, thus :-Keep a newt in a little water for three, four, or five days without changing the water. Its outer layer of cuticular epithelium is shed as a cast of the entire animal. Place the film in the solution for twenty-four hours, then wash in plain water till no colour is given off, 
and preserve in common alcohol till required. A small piece of this, stained in picro-carmine and mounted in Farrant's medium, shows the abovenamed structure beautifully. The fibres of white fibrous tissue may be shown to advantage, thus :Tear off fine strips of tendo achilles of an animal and place them for twenty-four hours in the solution. By "teasing" a little piece in water the white fibres held together by cement substance are well displayed.

\section{Osmic Acid.}

This is purchased as a 1 per cent. solution and diluted with distilled water to a $\frac{1}{2}$, a $\frac{1}{4}$ per cent. solution, \&c., as required. It is a hardening agent which also stains fatty matter, and is, therefore, useful in blackening the medullary nerve fibres. It is very poisonous, very expensive, and soon spoiled by exposure to light. It must be kept in a well stoppered bottle, which should be of black glass or covered with black paper, so that not a particle of light shall be admitted. Substances placed in it harden in from four to thirty-six hours, and may be prepared and mounted in either glycerine, glycerine jelly, or Farrant's medium. Very little pieces, not larger than half a grain of wheat, are to be used of any tissue, and the bottle in which they are placed must also be very small; the short glass tubes in which homœopaths keep their smallest pilules answer well.

With the above cautions and directions we may enumerate the tissues which are treated with advantage by osmic acid. In a 1 per cent. solution 
immerse costal cartilage of a kitten, puppy, or young rabbit, rat or guinea pig, for twelve hours; a piece of the sciatic nerve of the frog for ten minutes; the non-medullated nerve running in the wall of the splenic vein of an ox, also a piece of human placenta. A 1 per cent. solution may be injected into a testis with a hypodermic syringe, and the whole organ may then be placed in alcohol. The following are best treated with a $\frac{1}{2}$ per cent. solution, namely:-liver cells of a dog or small animal (the liver is cut across and the cut surface scraped) are placed in the solution for an hour. Areolar tissue is demonstrated by injecting a $\frac{1}{2}$ per cent. solution into the groin of a puppy or kitten immediately after death. A bulla is thus formed, which must be snipped out with scissors, spread on a slide stained with logwood and then covered. A $\frac{1}{2}$ per cent. solution may be forcibly injected into the anterior horn of a fresh spinal cord. The part is cut out, macerated for two days in dilute alcohol, and the multipolar cells isolated by "teasing," after staining with picro-carmine. A $\frac{1}{4}$ per cent. solution (the strength most frequently employed) may be used for showing mucous tissue; thus:-inject the axilla, or groin, of a very young embryo, as described above. Pieces the size of a pea of the following may be hardened in a $\frac{1}{4}$ per cent. solution: Submaxillary gland, fresh pancreas, pieces from each end of the stomach, small intestines, supra-renal capsules of the horse, human skin. Place a piece of the anterior horn of a fresh spinal cord for ten days in a $\frac{1}{10}$ th per cent. solution, then, after washing away the greyish deposit, place it in an equal 
quantity of glycerine and water for a fortnight; stain with weak magenta solution, "tease" in glycerine, and cover (Stirling).

\section{Miscellaneous Preparations. Salt Solution.}

This is made as a $\frac{3}{4}$ per cent. solution, that is, 75 grm. in 100 c.c. of water. It is used for washing away foreign matter from organs before immersing them in other fluids, and for examining fresh tissues. In so using it, a very small drop is placed on a slide, and in it is immersed a minute piece of the structure about to be examined. "Tease" this out with needles; then put over it a cover glass.

The following are to be studied:-Fresh columnar epithelium from the small intestines ; ciliated epithelium scraped from the roof of the frog's mouth; ciliary motion may be seen in the yellowish coloured gills of the common salt water mussel. Ligamentum nuchæ of ox; subcutaneous connective tissue; adipose tissue; red marrow from a long bone; striped muscle (best seen in sartorius of the frog); nerve fibre, sciatic of the frog; fresh pia mater; fresh spleen (the ox spleen by preference); thymus gland; kidney; Gasserian ganglion; placenta; decidua, \&c., may all be so treated.

\section{Glycerine.}

Margarine crystals may be obtained by steeping morsels of fat for twenty-four hours in glycerine. These post-mortem products appear as delicate needles. 


\section{Water.}

Squamous epithelium may be stained in magenta solution and examined in water. To obtain it, scrape the inside of the cheek with a blunt knife. The surface of the cheek may be also scraped and the scraping examined in water and afterwards irrigated with a 5 per cent. solution of liquor potassæ.

\section{Dilute Alcohol.}

Mix two parts of water with one of rectified spirit. This is a useful dissociating solution recommended by Ranvier.

Olfactory, ciliated and transitional epithelium, may be prepared as permanent preparations thus :Place a small piece of fresh trachea of a small quadruped for ciliated-a piece of fresh bladder for transitional-the head of a frog, with the nostrils slit up, for olfactory epithelium, in the solution for two days. The parts are then scraped and the scrapings stained and mounted in glycerine, or glycerine jelly. Stirling "fixes" the ciliated epithelium by placing the scraping in a 1 per cent. solution of osmic acid. Non-striped muscle may be obtained by taking out the bladder of a freshkilled frog and distending it with the solution, then placing it for twenty-four hours in the solution. After brushing away the mucous membrane with a camel-hair brush, stain the bladder in picrocarmine, and mount a piece in Farrant's medium. Isolated heart muscle fibres of the frog's and mammalian heart can be obtained by the same process. 
Purkinje's fibres can be obtained by snipping out bits of them (seen as fine transparent lines on the inner parts of the walls of the ventricles of the heart of oxen and sheep), and placing them in the solution for two days. The villi of human placenta can be isolated by soaking a piece of placenta two days in the fluid.

\section{Chromic Acid and Nitric Acid Solution.}

This is made by adding 1 c.c. of strong nitric acid to every 100 c.c. of the $\frac{1}{3}$ per cent. solution of chromic acid. It is used chiefly as a decalcifying agent.

Tooth, intervertebral disc with subjacent bone, articular cartilage with subjacent bone, costal cartilage of an old person, may each be placed in a large quantity of the fluid, which must be frequently changed, until the bony part is softened, which may be known by the needle test. The parts are washed free of the acids by frequent changes of water, then placed in weak, afterwards in strong, alcohol for preservation.

Permanent preparations of elastic tissue may be made thus:-Soak half-inch cubes of ligamentum nuchæ of ox or horse in this fluid for a week; wash free of acids and preserve in spirit, and make transverse and longitudinal sections in the usual way. (Stirling).

\section{Silver Nitrate.}

All animal tissues which are to be subjected to nitrate of silver and chloride of gold processes, must be perfectly fresh and carefully washed in 
distilled water before being treated by either of these re-agents.

Make a 1 per cent. solution of nitrate of silver in distilled water, keep it in a bottle carefully covered all over with black paper. This solution is to be further diluted to a $\frac{1}{2}$, a $\frac{1}{3}$, or a $\frac{1}{4}$ per cent. as required. Nitrate of silver is used to intensify and demonstrate the outlines and features of endothelium and cartilage cells. The cement substance between the cells absorbs the solution-all not absorbed must be carefully washed away with distilled water, and the tissue exposed to direct sunlight. Not the slightest taint of the unabsorbed silver solution must be allowed to remain upon the surface of the tissue, the staining of which would be spoiled by the black oxide granules of decomposed nitrate of silver. The tissues must be treated within a few minutes after death.

Omentum of rabbit.-Kill a rabbit by bleeding. Remove the omentum and wash it in distilled water; place it in a $\frac{1}{4}$ per cent. solution ten minutes; wash in ordinary water thoroughly; place the whole in a saucer containing water, and expose to diffuse daylight till slightly brown. Small pieces may now be stained with logwood and mounted in glycerine or glycerine jelly, and the logwood stain may be dispensed with if desired.

Septum Cysternce Lymphatica magnce of a frog. -Kill a frog and immediately open the abdomen. Gently push on one side the stomach, bowels, \&c., and pour distilled water on the part behind the stomach, when a delicate membrane floats up. Now pour a $\frac{1}{2}$ per cent. solution, drop by drop, over this till it becomes milky, and treat as above. 
Lungs of a kitten, or puppy.-Distend these with a $\frac{1}{2}$ per cent. solution immediately after death, then ligature the trachea, and sink them in alcohol till required.

Tendon.-Tendons and their sheaths are covered by endotbelium. Pinch the tail end off a recently killed mouse, and wash the fine tendon fibres thus drawn out in distilled water, and treat with a $\frac{1}{2}$ per cent. solution. Take another set of these fibres, pencil off their endothelium with a camel-hair brush dipped in distilled water, then silver. This shows the cell spaces in tendon.

Cornea.-The cell spaces of cornea may be shown by scraping away the epithelium from the anterior surface of the cornea of a pithed frog, then applying a 1 per cent. solution till greyish white. The cornea must be snipped at its edges, after being silvered, to make it lie flat on the slip. A rat's cornea may be likewise treated and stained with logwood.

Blood Vessels.-Kill a small quadruped by bleeding. Syringe out the blood vessels with distilled water; then inject a $\frac{1}{2}$ per cent. solution. Use the spleen, mesentery, and intestines. The spleen must be hardened in alcohol, and sections cut, exposed to light, after staining with logwood, or not, as desired. This shows the endothelium of the renous sinuses. The intestines must be cleared of their contents by distilled water; exposed to light in a saucer containing water; a piece of small or large intestine is snipped out, laid on a slide with its mucous membrane upwards, which is gently scraped away, the muscular and serous coats remain, and are to be mounted in balsam. 
Membranous Connective Tissue.-The omenta of an adult cat and rabbit, also of a young rabbit, are to be treated like the omentum of rabbit as already described, to show the membranous connective tissue. The omentum of the young rabbit shows developing fat cells and blood vessels. Some of the above should be stained with logwood.

Adenoid Tissue.-With a hypodermic syringe inject a fresh lymphatic gland with a $\frac{1}{4}$ per cent. solution, and after placing it for twenty-four hours in alcohol, make sections. These are stained with logwood, and exposed to light till brownish.

Cement Substance of Non-striped Muscle.-Wash out a small length of intestine of a rabbit with distilled water; then fill it with a $\frac{1}{2}$ per cent. solution, and tie both ends; then place it in a $\frac{1}{4}$ per cent. solution for a quarter of an hour. Wash away all the silver, and cover over with water in a saucer. Thin laminæ of the outer muscular fibres are to be stripped off with broad nibbed forceps. Mount some stained with logwood in balsam; others unstained, as desired. These also show the lymphatics.

Sciatic Nerve of a Frog.-To show Ranvier's crosses, kill a frog, and dissect out the sciatic nerve, wash it in distilled water, then place a piece a line in length in a $\frac{1}{4}$ per cent. solution for five minutes. Wash it now thoroughly in water and tease it carefully in glycerine, cover, and expose to light till brownish. Mount a small length of an entire thickness of an intercostal nerve of a rat or mouse, to show its endothelial covering. Of course it is not to be teased, and it must be very carefully washed or the endothelium will be destroyed.

Lymphatics of the Diaphragm. - Expose the 
posterior (ventral) surface of a rabbit or guineapig, immediately after death by bleeding, thus:Ligature the gullet and the posterior vena cava, then remove all the abdominal viscera. Tie up by the hind legs, then with a brush dipped in distilled water brush away all the epithelium covering the centrum tendinum of the diaphragm. Wash silver solution over it, and treat as already explained. This silvers the endothelia lining the lymphatics.

\section{Chloride of Gold.}

This salt is sold in small tubes containing 15 grains. A 2 per cent. solution is to be made with very pure distilled water, and kept in a black bottle, like the silver solution. The operator must also provide himself with formic acid and two or three fresh lemons.

Development of Capillaries.--Snip off the tail of a half-grown tadpole, place it in a watch glass, and squeeze the juice from a fresh lemon over it, and let it remain immersed for five or ten minutes; wash it in distilled water to remove all the juice; then steep it for half-an-hour in a 1 per cent. solution. Wash away the surplus gold, then place in a mixture of one part of formic acid and three parts of water for twenty-four hours in a cool place and away from the light. The gold chloride will then be reduced.

The following are to be treated in exactly the same manner :-

A whole Cornea.-That of a pig, dog, or cat, to show the nerves of the cornea, also the cornea corpuscles. 
A Piece of Skin.-'This should be taken from the snout of a pig or a mole, or both; also the soft part of a duck's bill. Sections show the nerves of skin.

Striped Muscle.-The recti muscles of a rabbit's eye are to be taken, a strip cut off lengthwise. 'These show nerve terminations in striped muscle.

Muscle with its Tendon.-Take a piece of the diaphragm of a rabbit; let the piece be part of the centrum tendinum with its attached muscle. Sections made in the long axis of the muscle fibres show the terminations of muscle in tendon; that is to say, the connecting links are shown.

Tendon from the Tail of a Mouse. - After treating a few leashes as above, snip off a piece a line in length; tease in glycerine and cover, or simply press the cover glass upon it till it flattens out. This preparation shows the relations of the cells and fibres in tendon.

Tail of Rat.-After treating as above, with the exception that it must remain an hour in the gold solution, decalcify, by placing in chromic and nitric solution, then make transverse sections and stain with logwood.

Nerve Ganglia.-Treat the heart of a frog, in small pieces, as above; dissect out the nerves and ganglia. These are also found lying along the course of the abdominal aorta of the frog, and may be taken from there if preferred. The nerve ganglia of the bladder and ureter of a small mammal may likewise be thus demonstrated. 


\section{The Injection of Blood Vessels.}

Blood and lymphatic vessels are more satisfactorily demonstrated when filled. The former are better injected with a fluid which becomes solid in ordinary temperatures. The vessels must be fully distended at the time and this distention must remain. Gelatine forms the foundation of injection masses for the above purposes, because it can be liquefied at blood heat and it solidifies in a little lower temperature; then, again, it is capable of being easily cut, and does not become brittle, but remains tough and resistant, though sufficiently soft. Injections of different colours are employed in order that the arteries may be distinguished from the veins, and these again from other channels, such as bile ducts, lymphatic vessels, capillaries, \&c.

The distribution of the capillary vessels, indeed, in many cases cannot be satisfactorily studied or demonstrated without injection or other special modes of preparation. Injections of single organs, or parts carefully dissected from the body of an animal, all the larger arteries and vessels being carefully tied, can be made; whilst the entire blood system of mammalian animals, including man, of birds and reptiles, can be injected from an arterial trunk, so that the finest capillary vessels shall be perfectly filled.

\section{Red Mass.}

This is made of gelatine coloured with carmine. In making it the greatest care must be exercised in 
order to ensure a neutral or at least a slightly acid mass; because if it should have any alkaline reaction, it will diffuse through the vessels, stain the adjacent tissues, and thus render the preparation altogether worthless. It is preferable that the mass should be slightly acid, but if too much so, granulation of the carmine will ensue, and the fluid will not be driven into the arterioles far less into the capillaries. Parts injected with a carmine and gelatine mass must be immersed in equal parts of water and methylated spirit, with 1 per cent. of acid added thereto.

\section{Dr. Carter's Carmine Mass.}

Take of Carmine, 60 grains.

Strong Ammonia, 120 minims.

Glacial Acetic Acid, 86 minims.

Solution of French Gelatine (gold label), 1 part to 6 parts of Water, 2 ounces.

Water, $1 \frac{1}{2}$ ounce.

Dissolve the carmine in the ammonia and water, filter if necessary. With this mix $1 \frac{1}{2}$ ounce of the hot solution of gelatine. Mix the acetic acid with the remaining half ounce of gelatine, and drop this mixture, very slowly, into the carmine and gelatine solution, with constant stirring.

\section{Dr. Stirling's Carmine Mass.}

Take of Carmine, 60 grains.

Strong Ammonia, 60 minims.

Glacial Acetic Acid, 80 minims.

Gelatine, 1 ounce.

Water, q.s.

Soak the gelatine in water for several hours; pour off the water, which is not absorbed, when the 
gelatine is completely saturated and swollen, and melt it in a water bath. Strain, whilst hot, through flannel and make up the solution to 2 ounces. Place the carmine in a mortar, add to it the ammonia and 2 ounces of water, and leave it for twelve hours. Filter, and add the acetic acid, drop by drop, stirring all the while, until the ammonia is completely neutralised. As the odour of the ammonia becomes faint, the acid must be added very cautiously. As long as there is free ammonia, the fluid is dull red, but it becomes of a brilliant colour as soon as the ammonia is neutralised. Now mix the two solutions at a temperature of $40^{\circ} \mathrm{C}$.

\section{Dr. G. Sims Woodhead's Mass.}

Take of Carmine, 4 parts by weight.

Liq. Ammonia, 8 parts by measure.

Gelatine (Cox or Coignet's), 10 parts by weight.

Distilled Water, 100 parts by measure.

Put the carmine in a mortar, and pour on the ammonia, when an almost black paste will be formed if the carmine is pure; pour in the water, and set the solution aside to be filtered. Place the gelatine in a narrow glass jar, and add sufficient distilled water to cover it, and allow it to stand until the gelatine is thoroughly softened. Warm the carmine solution in a pan of water, kept at nearly boiling point over a Bunsen gas burner, and add the gelatine; stir thoroughly, add a 10 per cent. solution of acetic acid, drop by drop, until the alkalinity of the ammonia is neutralised, and the fluid shall be even slightly acid. The point at which this takes place will be recognised by the pungent odour of the ammonia becoming fainter and 
fainter, and that of the acid substituted, whilst the fluid loses its bright carmine, transparent colour, and turns a dull brownish-red.

\section{Blue Injection Mass.}

Take of Soluble Prussian Blue, 4 drachms.

Gelatine, 4 ounces.

Distilled Water, 20 ounces.

Thoroughly mix the blue powder in a mortar with half the water. Treat the gelatine as directed in the case of the red mass, and add the blue solution very gradually, and with constant stirring, to the liquefied gelatine. Filter through fine flannel. The greatest care must be exercised that no trace of alkali shall enter into the composition of this mass-all the vessels used in making it should be carefully washed with acidulated water, and the purest distilled water must be used in its preparation. Its colour is liable to fade and sections injected with it, especially when mounted in balsam, lose their colour after a time. It is better, therefore, to mount all specimens injected with Prussian blue in a slightly acid medium-glycerine jelly prepared with boracic acid-pure glycerine with a small proportion of acetic acid, or Farrant's medium are the best preservatives for these injections.

\section{Blde Injection Mass.}

(Robin's modification of Dr. Beale's Prussian Blue) is made as follows :-

Take (a) Sulphocyanide of Potassium, saturated solution 90 c.c.

Glycerine, 50 c.c.

(b) Liquid perchloride of Iron at $30^{\circ}, 3$ c.c.

Glycerine, 50 c.c. 
Mix slowly, and combine the mixture with three parts of a glycerine-gelatine injection mass made as follows (Robin's formula):

Dissolve, in a water bath, 50 grammes of French gelatine (gold label) in 300 grammes of distilled water in which some arsenious acid has been dissolved; add 150 grammes of glycerine and a few drops of carbolic acid.

Scheele's Green Injection Mass (Robin's formula).

Take of Arseniate of Potash (saturated solution), 80 c.c.

Glycerine, 50 c.c.

and of

Sulphate of Copper (saturated solution), 40 c.c.

Glycerine, 50 c.c.

Mix and combine with three parts of the glycerine gelatine injection mass as in the preceding formula.

\section{Aqueous Injection Fluids.}

Carmine. (Emery's formula.)

To a 10 per cent. ammoniacal solution of carmine add acetic acid, with constant stirring, until the colour of the solution becomes brilliant red. After the precipitation of the carmine, pour off the supernatant fluid and inject the clear solution. Place the injected specimens in strong alcohol to fix the carmine.

Carmine. (Gerlach's formula.)

Carmine, 77 grains.

Distilled water, 70 grains.

Liquor ammoniæ, 8 minims.

Dissolve the carmine in the ammonia and water, and leave the solution exposed to the air until all 
the ammonia has evaporated. Dissolve a drachm and a half of best gelatine in a drachm and threequarters of water; add a few drops of glacial acetic acid. This fluid is to be injected warm.

\section{Acid Carmine Fluid. (Dr. Beale's formula.)}

Carmine, 5 grains.

Glycerine, with 8 to 10 minims of acetic acid, $\frac{1}{2}$ ounce.

Glycerine, 1 ounce.

Alcohol, 2 drachms.

Distilled water, 6 drachms.

Ammonia, a few drops.

Mix the carmine with a few drops of water, and when thoroughly incorporated, add 5 minims of liquor ammonice. Add about half an ounce of the glycerine, and shake the mixture well in a bottle. Next, pour in very gradually the acid glycerine, frequently shaking the bottle during admixture. Test the fluid from time to time with blue litmus paper, and if not of a very decidedly acid reaction, add a few drops more acid to the remainder of the glycerine and mix as before. Lastly, add the alcohol and water, very gradually, shaking the bottle thoroughly after the addition of each successive portion, until the whole is well mixed.

\section{Prussian Blue Fluid. (Dr. Beale's formula.)}

Wood-naphtha, or pyro-acetic spirit, $1 \frac{1}{2}$ drachm. Glycerine, 2 ounces.

Alcohol, 1 ounce.

Ferrocyanide of Potassium, 12 grains.

Tincture of Perchloride of Iron, 1 drachm.

Distilled Water, 3 ounces.

Dissolve the ferrocyanide of potassium in 1 ounce of the glycerine, mix the tincture of iron 
with the other ounce. Put this into a bottle. Add the iron mixture, drop by drop, to the ferrocyanide solution, shaking all the time. Mix the naphtha with the alcohol, add the water very gradually, and thoroughly shake the whole.

Prussian Blue acidulated Glycerine Fluid.

$$
\text { (Dr. Beale's formula.) }
$$

Price's Glycerine, 2 fluid ounces.

Tinct. of Sesquichloride of Iron, 10 minims.

Ferrocyanide of Potassium, 3 grains.

Strong Hydrochloric acid, 3 minims.

Distilled Water, 1 ounce.

Dissolve the ferrocyanide in one half of the glycerine, and the sesquichloride of iron in the other, add the latter drop by drop to the former. Finally add the water and the acid, and to the whole add 2 drachms of alcohol.

Neutral Glycerine Prussian Blue Fluid.

(Dr. Beale's formula.)

Glycerine, 1 ounce.

Alcohol, 1 ounce.

Ferrocyanide of Potassium, 12 grains.

Tincture of Perchloride of Iron, 1 drachm.

Distilled Water, 4 ounces.

Dissolve the ferrocyanide in one ounce of the water and glycerine, add the tincture of iron to another ounce. Mix these solutions very gradually, in a bottle, and shake thoroughly. The iron is to be added to the ferrocyanide solution. Lastly, the alcohol and the remaining water are to be gradually added, with constant shaking of the bottle.

Specimens injected with this fluid should be preserved, and mounted, in acidulated glycerine. 
Neutral Glycerine Turnbula's Blue Fuuid.

(Richardson's formula.)

Ferridcyanide of Potassium, 10 grains.

Sulphate of Iron, 5 grains.

Distilled Water, 1 ounce.

Price's Glycerine, 2 ounces.

Alcohol, 1 drachm.

Proceed as in the last formula. Mix the sulphate of iron in 1 ounce of the glycerine, and the ferridcyanide in a small quantity of water, and mix this with the other ounce of glycerine. Gradually mix these two solutions, with constant shaking, and add the alcohol.

Turnbull's blue is not so liable to fade as Prussian blue. 


\section{CHAPTER IV.}

\section{On Injecting Blood Vessels, \&c.}

IT has been generally, though erroneously, considered that a perfect injection of an animal cannot be secured until the rigor mortis has passed away; it is, however, indubitable that the most successfu] injections have been made immediately after death.

Dr. Beale ${ }^{1}$ says: "I have found that most perfect injections may be made before the muscular rigidity begins, that is, within a few minutes after the death of the animal. Most of my fine injections have been made less than five minutes after death, and in the case of very young animals, so complete has been the injection of the capillary vessels, that where the capillary has not been fully developed, the injection has filled the pervious portion, and has penetrated to the very spot where the tube was commencing to be formed." This the author can entirely corroborate.

The ordinary process for injecting blood vessels is with the syringe; as this is a very delicate opera114.

"How to Work with the Microscope," fifth ed., 1880, p. 
tion requiring much dexterity of manipulation, considerable practice, some experience and great care, the student must not be discouraged by disappointments and failures, but persevere until success is attained. It is advisable to practise with cold aqueous fluid media, and this operation being successfully accomplished, to go on to injections with the warm gelatine "mass." It will be found best, also, to commence by injecting the organs of animals, such as the kidney, liver, lung, large and small intestines, \&c., of the cat, the sheep, or the pig, the eye of an ox (from the artery), and by complete injections (from the aorta), of small animals, e.g., rat, guinea-pig, rabbit and frog, before attempting to inject a whole animal.

The kidney should be singly injected with carmine mass through the artery, and a double injection should also be made with carmine from the artery, and with blue from the vein.

The liver in like manner may be injected from the portal and hepatic veins and from the bile ducts -(thus-ligature the common bile duct, remove the fundus of the gall bladder, remove as much bile as gentle pressure will force out, affix the cannula, and inject).

In injecting isolated organs removed from the body of an animal, the greatest care must be taken to tie all divided vessels, and to use no more than the necessary pressure upon the piston of the syringe. The slower and more gradual the flow of the fluid, the more perfect will be the injection.

All animals, or "parts" to be injected with a gelatine mass must be placed in warm water of a temperature from $40^{\circ}$ to $45^{\circ} \mathrm{C}$. during the process. 
To Inject an Animal with a Gelatine "Mass" BY THE Syringe.

Arrange-so that they may be ready to handthe necessary instruments and apparatus, viz., the syringe, thoroughly cleaned and tested for work, with its stopcocks and cannulæ, scalpels of several sizes, a large and a small pair of scissors, dissecting forceps, "bull-dog" forceps (for clamping leaking vessels), a curved needle threaded with silk or thread for tying vessels, a wash-bottle of a capacity of from half a pint to a pint. The injection mass is to be melted by standing the vessel containing it in hot water, and it is to be kept fluid in a water-bath of a temperature from $40^{\circ}$ to $45^{\circ} \mathrm{C}$.

Chloroform the animal (say a rabbit) which is to be injected, and make an L-shaped incision into the thorax so as to expose the heart and aorta. This is done by cutting upwards along the middle line of the sternum nearly as far as the root of the neck, then making a second incision at right angles to this, to the rabbit's left. A triangular flap is thus made and the heart, enclosed in the pericardium, exposed. Having cut through the pericardium, seize the apex of the heart with a pair of forceps and snip it off. The right and left ventricles are thus opened, and the animal instantly bleeds to death. The opening in the right ventricle, leading to the pulmonary artery, has a crescentic, slit-like appearance, whilst the opening in the left ventricle, leading to the aorta, is circular. Insert the cannula into the aorta and tie it in securely with a silk ligature. Wash all blood from the cavity of the thorax in order to ensure the cleanliness of the 
water-bath. Now place the rabbit into a warm water-bath of a temperature of from $40^{\circ}$ to $45^{\circ} \mathrm{C}$., and let it remain for from 10 to 15 minutes, i.e., until thorougly warmed. The entire abdomen may be carefully opened along the middle line to allow the warm water to surround the internal organs, and to ensure the equable flow of the injected fluid into all parts of the body.

When the animal is thoroughly warm-the syringe, which should also have lain for some time in warm water-is to be carefully filled with the injecting fluid, in which its nozzle is to be entirely immersed, whilst the piston is worked several times to ensure the expulsion of all air. It is then placed in the stopcock, which should, however, first be filled with fluid from the syringe to prevent the introduction of any air, and which has already been inserted into the neck of the cannula to receive the nozzle of the syringe, and which, stopcock, is to be turned off each time it may be necessary to refill the syringe; now with a gentle equable pressure the fluid is to be injected into the animal until the entire system of blood vessels shall be filled, but not distended. The operator from time to time examines the head, opens the eyes and parts the lips of the animal to watch the progress of the work. If the fluid is flowing properly, the mouth will almost immediately exhibit signs of the colour with which the injection is being made, and which will first be seen in the gums of the lower incisor teeth. When the eyeballs show the finest lines of colour traversing them, the capillaries may be considered to be filled and the injection complete and perfect.

The animal is now to be put into cold water in a 
large vessel, and placed under a tap which is to be allowed to drip slowly upon it for two hours; it may then be dissected and the organs placed in 70 per cent. methylated spirit for twenty-four hours and then transferred to strong methylated spirit in which they are to be preserved.

If it is desired to inflate the lungs, remove them entire; very carefully tie all vessels and let them remain in the warm water. Melt together and keep fluid in a warm water-bath, two parts of cacao butter and one part of lard, suspend the lungs in the water-bath, insert a funnel into the trachea, and pour the butter and lard gradually into the lungs until they are fully inflated-but no moregreat care must be exercised not to over-distend them. Now place them in cold water and afterwards into spirit as already described.

Dr. Fearnley has devised an elaborate and most effective modification of Ludwig's constant pressure apparatus for making injections, which he fully describes in his "Practical Histology," and which ensures such constancy, accuracy and delicacy in the pressure upon, and flow of, the injecting fluid, that there can be no comparison between its almost automatic action and that of the syringe worked by a piston.

This apparatus consists of a bath, having a shallow part to receive the animal to be injected, and a deeper part for the vessel containing the injection fluid to stand in. A large (40 ounce) Wolff's bottle, with three necks, is fitted with three

1 "A Course of Elementary Practical Histology," by William Fearnley, p. 70. 
perforated india-rubber stoppers. A glass tube reaching nearly to the bottom of the bottle passes through the central stopper; each of the other stoppers is fitted with a glass tube projecting but little beyond the stopper into the bottle, but pro-

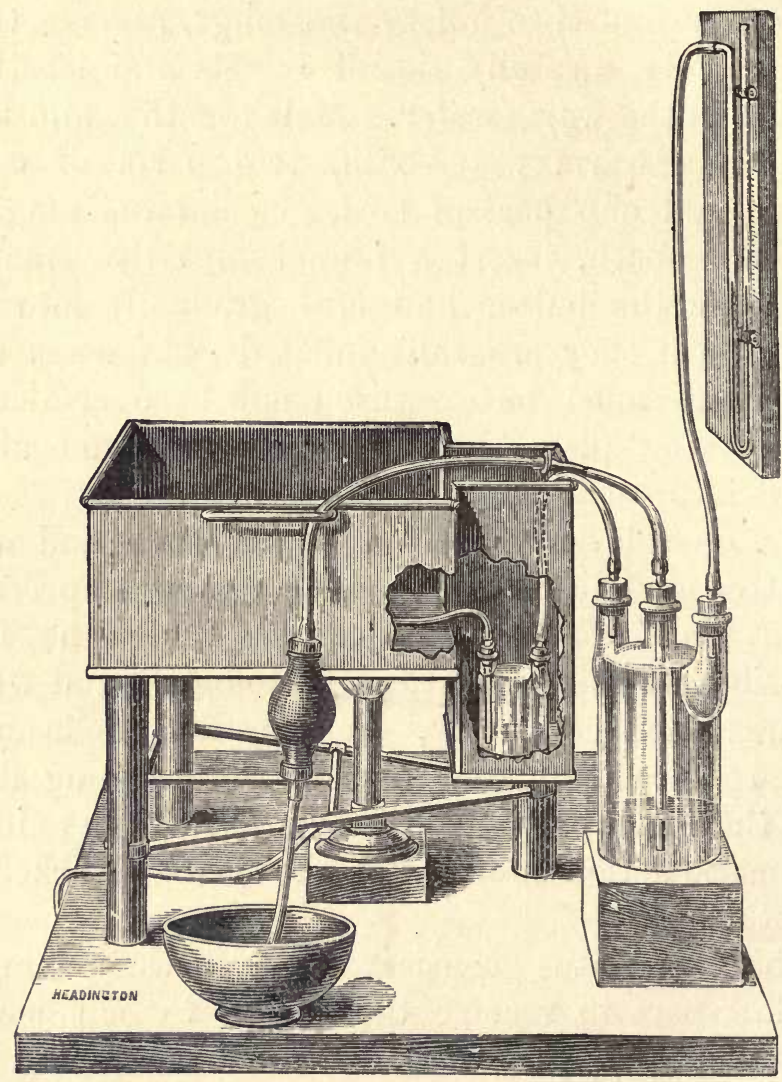

jecting sufficiently above the stopper to admit of a piece of india-rubber tubing, of the diameter used for infant's feeding bottles, being fixed upon it.

A basin, or still better, a water-bottle, of a 
capacity of a litre or more, a mercurial manometer, a Higginson's syringe, a spirit lamp, or a Bunsen's gas burner, some glass and brass cannulæ, and some india-rubber tubing, two clamps (one for the pressure tube, the other for the delivery tube), will also be required.

To make an injection of an animal with a gelatine mass, proceed as follows :-

Fill the water-bath with water of a temperature of from $40^{\circ}$ to $45^{\circ} \mathrm{C}$. Keep it at this temperature by placing a spirit lamp or a Bunsen gas burner under the bath. Melt the gelatine mass and pour it, through flannel, into the two-stoppered Wolff's bottle. Place this bottle in the place provided for it in the bath-the bottle should be weighted to prevent its floating as the fluid is withdrawn from it. Arrange the manometer, pressure bottle, syringe and basin (or water-bottle), as shown in illustration. Now chloroform the animal to be injected, and proceed as already described; affix the cannula, tie it securely into the aorta, and after washing away all blood from the thorax, \&c., place the animal in the water-bath. The animal must be submerged in the water, but not too deeply. Clamp the delivery tube of the bottle containing the injection fluid and, with the syringe, pump into the pressure-bottle-from the basin or water-bottle-sufficient water to raise the mercury in the manometer one inch; remove the clamp from the delivery tube, allow the air to escape from it, and immediately connect it with the cannula. The tube must remain submerged whilst the connection is being made. Now keep up an equable pressure by means of the syringe, and when the mercury in the manometer shall have risen to 
from 4 to 5 inches, it will be found that the animal is completely injected. The head must be examined from time to time, as already described, so that the rising colour of the lips, gums, tongue and eyes may be watched. The animal is now removed to cold water, and treated as directed above.

This method of applying pressure is wonderfully delicate and equable; it will be found that whilst the mercury in the manometer can be raised almost imperceptibly, one entire compression of the barrel of the syringe raises it 1 inch. 


\section{CHAPTER V.}

\section{On Staining Fluids, and Staining.}

The value of the various staining processes consists in the perfection with which the differentiation of the tissues is accomplished, and their features and structure brought out. In the staining of tissues it is all important that the nuclei of the cells shall be deeply stained, whilst the cellular structure is clearly defined.

Staining is an art, requiring much experience and great delicacy of manipulation. It is one thing to colour a section or a tissue, quite another to stain, and especially to double-stain and differentiate it. In double and triple staining the great desideratum is to secure a thoroughly good nuclear stain as the ground colour, and to supplement this with a delicate tint, or tints, which shall prove a good contrast to the first stain and thoroughly differentiate the tissue without diffuseness.

For staining the nuclei of the cells, for beauty and permanence, no staining fluids have yet been discovered to surpass hæmatoxylin, carmine and picro-carmine. The brilliancy of carmine has its advantages in many cases, whilst the delicacy, the 
beautiful gradations of tints, from the deepest violet to the most delicate blue, which by careful treatment are produced by a good logwood stain, give a sense of relief to the eye during prolonged observations which no other tints, perhaps, afford. No more beautiful stainings have been produced than those obtained by a prolonged immersion (say for twelve hours) in a logwood solution, made according to the first formula given below, and diluted to a light violet colour, which after the sections so stained have lain in tap water for twentyfour hours will result in a most beautiful blue ground colour with the nuclei of a darker blue. Let them then be placed in spirit for half an hour, and then in a stain made from 2 parts of a saturated alcoholic solution of rubin and 1 part of a saturated alcoholic solution of yellow eosin diluted with alcohol, until it becomes of a delicate pink shade, and in which the sections may be kept until they are "cleared" and mounted. The sections thus treated will be found to be distinctly triplestained in blue, crimson and orange colour without diffuseness and with perfect differentiation, and if mounted in "xylol balsam" they will prove quite permanent.

Carmine and picro-carmine also are invaluable as nuclear stains, whilst for many tissues, and especially for most vegetable tissues, they are preferable to logwood, and, when mounted in a suitable medium, sections stained with them prove equally beautiful and permanent preparations.

A carefully selected list-of formulæ for the most important and reliable staining re-agents is here appended, the proper media in which sections 
stained by them should be mounted being given with each formula.

\section{Hzmatoxylin Staining Fluids.}

1. Hæmatoxylin (Crystals), 1 gramme.

Alum-powdered, 10 grammes.

Distilled Water, 100 c.c.

Alcohol, 5 c.c.

A small piece of Camphor.

This is a splendid nuclear stain.

It does not become active until from two to three months after it is made. It improves by keeping, and remains perfectly good for two or three years. It must be filtered each time it is used, and may be used over and over again. Its full strength is too strong for use; for rapid staining, i.e., in from five to ten minutes, add to it an equal volume of distilled water. For slow staining, dilute it until its colour becomes a light violet, and leave the section in the stain thus diluted for six to twelve hours. Overstained sections can be decolourised to any desired tint in distilled water 5 parts, acetic acid 1 part.

After staining and, if necessary, decolourising the sections, place them in tap water for twentyfour hours, and they will be found of a beautiful blue tint. Mount in xylol balsam.

\section{Ehrlich's FormulaA.}

(a) Hæmatoxylin, $1 \frac{1}{2}$ to 2 grammes. Absolute Alcohol, 100 c.c.m.

(b) Glycerine, 100 c.c.m.

Distilled Water, 100 c.c.m.

Glacial Acetic Acid, 5 c.c.m.

Alum Sulph. (powdered), to saturation.

Mix the two solutions. 
Allow it to stand for about six weeks, occasionally shake it, when it will have obtained its greatest depth of colour, then filter it once for all. It will then stain in about half an hour or less and does not overstain. But better results are obtained by adding a few drops to an ounce of distilled water and leaving the sections therein for from three to four hours. It keeps well, and as it gets older, stains more rapidly. If its action becomes too rapid or diffuse, add more acetic acid. It may be used over and over again.

Mount in xylol balsam.

\section{Weigert's Formula.}

(a) Hæmatoxylin (Crystals), 1 gramme.

Absolute Alcohol, 10 c.c.

Distilled Water, 100 c.c.

(b) Ferridcyanide of Potassium, $2 \frac{1}{2}$ grammes.

Borax (powdered), 2 grammes.

Distilled Water, 100 c.c.

Place the sections in absolute alcohol for one hour then in solution (a) until they become quite black - then place them in distilled water for a few minutes. Decolourise in solution $(b)$ until they are of a dark brown.

This does not keep good and active beyond a few months. Filter each solution every time it is used, and it may be used several times. Mount in xylol balsam. This is a very fine nuclear stain, and brain, spinal cord, nerves, \&c., are superbly stained by it. It is also a splendid stain for micro-photographic purposes, as the beautiful shades of brown which carefully stained sections exhibit give very fine photographic results. Any animal or vegetable 
sections, of which it is desired to produce lantern slides or micro-photographs, may be stained by this process. Mount in xylol balsam.

\section{Kleinenberg's Formula.}

(a) Prepare a saturated solution of chloride of calcium in alcohol (of 70 per cent.), add a small quantity of powdered alum, filter, mix 1 part of this with 8 parts of 70 per cent. alcohol. .

(b) Make a saturated solution of hæmatoxylin crystals in absolute alcohol. When using this for staining purposes add to a small quantity of solution $(a)$, in a watch glass or saucer, sufficient of (b) to give it the desired tint.

This is a very fine nuclear stain, and permanent, but the stain itself will not keep very long; all sections or tissues to be stained in it must be thoroughly soaked in distilled water so that no trace of acid may remain in them.

Slow staining in a very dilute solution gives the best results. Mount in xylol balsam.

\section{Mitchell's Formula.}

Take of finely ground Logwood, zii.

Alum Potash, 3ix.

Glycerine, fl. ziv.

Distilled Water, quant. suff.

Moisten the logwood with water until it is quite damp. Place it in a large funnel with filter paper and then pass ordinary water through it until the liquid runs away almost colourless. When all the water has drained from it, place the logwood on a large dish to dry.

Dissolve the alum in 8 ounces of distilled water, 
place the logwood in a vessel of sufficient size and pour the alum solution upon it, allow it to remain for forty-eight hours, stir it occasionally, strain it off; add the glycerine and filter. Add 5 per cent. of absolute alcohol to it, and keep it in a thoroughly well stoppered bottle. For rapid staining this fluid may be used full strength, but better results will be obtained from prolonged immersion, say for twelve hours, in a weak solution. This stain will give a delicate violet tint to the sections with beautiful clearness and differentiation. Mount in xylol balsam.

\section{Carmine Staining Fluids.}

1. Ammonia Carmine. (Dr. Beale's Formula.)

Carmine, 10 grains.

Liquor Ammonia fort., B.P., $\frac{1}{2}$ drachm.

Price's Glycerine, 2 ounces.

Distilled Water, 2 ounces.

Alcohol, $\frac{1}{2}$ ounce.

Dissolve the carmine in the ammonia by means of heat. Boil for some seconds and allow it to cool. Let the ammonia evaporate for an hour or more, and when the odour is nearly gone, add the glycerine, water and alcohol, and filter. When, from keeping, the carmine is precipitated, add one or two drops of ammonia. It may be used full strength or diluted with alcohol, which will increase its penetrative power. This is a fine, nuclear stain.

Mount in xylol balsam.

2. Ranvier's Formula.

Distilled Water, 100 parts.

Ammonia, 1 part.

Carmine, 1 part. 
Rub up the carmine in a mortar with a small quantity of the water, add the ammonia, and when the carmine is dissolved, add the remainder of the water. If there is an excess of ammonia, use heat until the carmine begins to be precipitated.

Mount in xylol balsam.

\section{Huxley and Martin's Formula.}

Carmine, 2 grammes.

Liquor Ammoniæ fort., B.P., 4 c.c.

Distilled Water, 48 c.c.

Dissolve the carmine in the ammonia and water. Place it in a bottle without a stopper until all odour from the ammonia has nearly passed away. Keep it in a thoroughly good stoppered bottle. When used it should be diluted with from fifteen to twenty times its bulk of water.

Mount in xylol balsam.

4. Borax Carmine Fluid.

(Foster and Balfour's Formula.)

Carmine, 3 grammes.

Borax, 4 grammes.

Distilled Water, 97 grammes.

Mix, apply heat with constant stirring until the liquid nearly boils, set it aside to cool and when cold, add

$$
\begin{aligned}
& \text { Alcohol (Rectif.), } 70 \text { c.c. } \\
& \text { Distilled Water, } 30 \text { c.c. }
\end{aligned}
$$

Shake it up thoroughly, let it stand for thirty-six to forty-eight hours, and filter it.

Mount in xylol balsam.

Specimens stained in these carmine fluids may be 
rendered more brilliant, and any diffuseness removed by immersion in

Hydrochloric Acid, 1 per cent. in Distilled Water, 1 part.

Alcohol (Rectif.), 2 parts.

Picro-Carmine Staining Fudids. (Gage's Formula.)

1. Take of carmine and of picric acid equal parts by weight. Dissolve the picric acid in 100 times its weight of distilled water. Heat may be used if necessary. Dissolve the carmine in 50 times its weight of liquor ammonia fort., B.P.

Mix the two solutions, filter through thick filterpaper, evaporate the filtered liquid to dryness, dissolve the filtrate in 100 times its weight of distilled water. Filter until a perfectly clear solution results. Then add to each 100 c.c. of the solution,

25 c.c. of Glycerine (Price's).

10 c.c. of Absolute Alcohol.

This will keep perfectly well, but should be filtered at intervals of a few months.

\section{Weigert's Formula.}

Soak 2 grammes of carmine for twenty-four hours in 4 grammes of ammonia. Place it under a glass shade to prevent evaporation, add 200 grammes of a very strong solution of picric acid, and replace it under the glass shade for a second period of twenty-four hours. Then add, very gradually, acetic acid until precipitation commences. Again put it aside for twenty-four hours. Filter, then add ammonia in small quantities-drop by drop-at intervals of twenty-four hours until the 
solution becomes clear. If this fluid when used overstains yellow, add acetic acid, if red, add ammonia.

\section{Picro-Lithiom Carmine.}

Dissolve $2 \frac{1}{2}$ grammes of carmine in 100 c.c. of a saturated solution of lithium carbonate, add 2 to 3 c.c. of a saturated solution of picric acid. Filter.

All preparations stained with picro-carmine may be mounted in xylol balsam, but Farrant's medium is preferable.

\section{Picro-Carmine and Eosin. (Lang's Formula.)}

Mix 50 parts of a 1 per cent. picro-carmine solution with 50 parts of a 2 per cent. aqueous solution of eosin; after prolonged immersion-for from twelve hours to forty-eight, or even seventytwo hours-wash out the picrin in repeated changes of alcohol, until the eosin ceases to colour the spirit.

This is a very fine double stain.

Mount in xylol balsam.

\section{SUlph-Indigotate of Soda.}

Make a saturated solution of sulph-indigotate of soda in distilled water, and to each ounce thereof add from 3 to 6 minims of rectified alcohol, filter, and keep in a closely stoppered bottle. It is better to make small quantities of this fluid as required, as it does not keep very long. This stain is suitable only for fibrous tissues-skin, scalp, hair, and for epitheliomatous growths.

Mount in xylol balsam. 


\section{Cochineal Staining Fluid.}

Alcoholic Cochineal. (Mayer's Formula.)

Macerate cochineal powder in 70 per cent. alcohol for some days, in the proportion of 10 c.c. alcohol to 1 gramme of the cochineal.

Stir frequently and filter.

Saturate the specimens before staining them with 70 per cent. alcohol, and use alcohol of that strength for all the processes connected with the use of this stain, and for diluting it. After staining the specimens, wash them in repeated changes of the alcohol until they cease to tint it.

\section{Alum Cochineal. (Czoker's Formula.)}

Pulverise in a mortar, cochineal 7 grammes, and dried alum 7 grammes; add 700 c.c. of distilled water, and boil down to 400 c.c. When cool add 1 per cent. of carbolic acid crystals; filter several times. It will remain fit for use for about six months, when it must again be filtered, and a small additional quantity of carbolic acid added to it.

Use it full strength, it does not over stain however long the specimens are left in it.

The specimens must be immersed in it for at least two hours, and may require to remain immersed from three to six hours.

It acts as a double stain, colouring the nuclei violet and the tissues red.

\section{Anilin Blue Black.}

Dissolve 1 decigramme in 4 c.c. of distilled water, to this add 100 c.c. of rectified alcohol, and keep the solution in a well-stoppered bottle. 
Stain the specimens in this fluid much diluted with spirit. The stained specimens may be kept, to be mounted as required, in spirit to which a small quantity of this fluid has been added.

This is a most valuable stain for brain, spinal cord, nerve centres, and the nervous system generally, also for bone and cartilage.

Mount in xylol balsam.

\section{Anilin Dyes as Staining Fluids.}

'These are valuable for many purposes, and have many useful properties. They stain rapidly, with great clearness and brilliancy, and are especially useful as double and triple stains in conjunction with hæmatoxylin or carmine.

No more beautiful or effective staining process has been discovered than that in which the specimens, having been stained in hæmatoxylin fluid subjected to the action of "tap" water for twentyfour hours, until they have acquired a beautiful blue tint, are afterwards stained in a weak solution of 2 parts fuchsin and 1 part yellow eosin.

This process gives a clear and pure triple stain.

The student will find by experiment many equally useful and beautiful combinations of these dyes.

It has always been affirmed that most of the anilin colours are more or less, and indeed, more rather than less, fugitive, but the author ventures to affirm as the result of his long experience that if the necessary precautions are taken there is no reason whatever why specimens stained with anilin colours should not remain as permanent as any others, and he has in his collection preparations so 
stained, made twenty years ago, which are as perfect as on the day they were mounted.

Since xylol has been introduced anilin stains may be used with even greater confidence, as that preparation tends rather to preserve than to destroy the anilin colours, which are undoubtedly adversely affected by benzol. Xylol, therefore, should be used to clear the specimens before mounting them, and Canada balsam, which has been thoroughly hardened and re-dissolved in xylol, should be used as the mounting medium. If the specimens, having been cleared in a mixture of equal parts of xylol and absolute phenol, are mounted in "xylol balsam," brilliant clearness will result, and the preparations will prove absolutely permanent.

The author has found it most convenient to make saturated solutions in alcohol of the anilin colours given in the appended list, and to stain the specimens slowly in weak solutions, either alcoholic, made by adding a few drops of the saturated solution to alcohol, or aqueous, made by adding a small quantity of the stain to distilled water. The advantage of using alcoholic solutions is that when the dilute stain has been found to give the desired tint it may be preserved for use over and over again. It must be filtered each time, and as its colour becomes lighter, must be restored to its original tint by the addition of a few drops of the "stock" solution.

The following will be found the most generally useful of the anilin colours and sufficient for all purposes.

Anilin Blue.

Nicholson's Blue. 
Soluble Blue.

Bismarck Brown.

Chrysoidin.

Eosin (Red).

Eosin (Yellow).

Fuchsin.

Gentian Violet.

Acid Green.

Iodine Green.

Magenta.

Methyl Green.

Methyl Violet.

Orange.

Rubin.

Saffranin.

Spiller's Purple.

Vesuvin.

Bismarck Brown.-Make of this (and of all the above) a saturated solution in alcohol (all these solutions should be filtered at intervals of a few weeks). Add a few drops to a small staining saucer full of spirit, and place the specimens in it for a few minutes. Remove them to alcohol, clear in xylol and phenol and mount in xylol balsam.

If it is desired to mount the specimens in Farrant's medium, or glycerine, wash them first in alcohol, then in distilled water. Bismarck Brown does not overstain, nor does it wash out readily either in water or alcohol. It is suitable for bacteria and micrococci, and forms a very good double stain in conjunction with other colours : its brown tints render it a good stain for preparations intended for micro-photography.

Eosin.-Both red and yellow eosin are most use- 
ful stains either singly or in conjunction with other staining fluids, and especially with hæmatoxylin; whilst, as before stated, yellow eosin combined with fuchsin gives, with hæmatoxylin as the ground stain, a precise and beautiful triple stain.

Eosin has great staining and especially penetrating powers, and specimens may remain in a weak alcoholic solution for an indefinite time without fear of their becoming over-stained. No trace of acid must remain in any medium in which eosin stainings are mounted; but preparations mounted in xylol balsam are practically permanent.

Epithelium is beautifully stained by eosin and the blood of the frog or triton double stained with hæmatoxylin and eosin forms a superb preparation.

Iodine Green.-Add to distilled water, in a staining saucer, sufficient of the saturated solution in alcohol to give it a fairly deep tint. Place the specimens in distilled water for a short time before staining them. Iodine green stains very rapidly, and in many cases instantaneously. Wash them in water, dehydrate in absolute alcohol, clear in xylol and phenol, and mount in xylol balsam. Stained specimens may remain in a weak alcoholic solution of this dye indefinitely and be mounted as required.

Methyl Green.-This is an intense nuclear stain, and Dr. Curschmann states that it has "a peculiar affinity for amyloid substance, colouring it an intense violet; whilst surrounding tissues that have not undergone degeneration are stained green or bluish green."

Methyl Violet is also a valuable stain for tissues which have undergone amyloid degeneration: the amyloid substance is stained red, and the unaffected parts violet. 
Orange and Rubin.-These combined, in about the proportion of 1 part of the former to 2 parts of the latter, give, in conjunction with hæmatoxylin as the ground stain, a beautiful triple stain.

Saffranin is a good nuclear stain, imparting a beautiful rose tint to the tissues, and staining the nuclei a very deep red.

Spiller's Purple is also a good nuclear stain, and is a very restful colour for the eyes.

Vesuvin, in conjunction with anilin blue, is very effective as a stain for bacilli and bacteria.

Specimens stained with anilin dyes must not be cleared in oil of cloves or cedarwood oil, but exclusively in xylol and absolute phenol in exactly equal proportions. This medium clears almost instantaneously and perfectly, having also a preservative rather than a destructive effect upon the anilin colours of which it increases the brilliancy; whilst, moreover, the xylol balsam, in which they should be mounted, exercises a preservative effect upon the preparations, and which it renders practically permanent.

(Hæmatoxylin crystals, and the anilin dyes, are supplied of the finest quality by Dr. G. Grübler, of Leipzig, and can be obtained from his agent, Mr. C. Baker, 244, High Holborn, London, W.C.)

\section{The Staining of Bacilli and Bacteria.}

To stain bacillus tuberculosis :

Formula No. 1.

1. R Fuchsin, 1 gramme.

(a) Absolute Alcohol, 10 c.c.

Carbolic Acid, 5 grammes.

(b) Distilled Water, 100 c.c. 
Mix the two solutions. about two months.)

(This remains active for

2. Anilin (or Methylene) Blue, 2 grammes. Absolute Alcohol, 15 c.c.

Distilled Water, 85 c.c.

(This will keep a long time.)

Spread a thin layer of sputum on a thin glass cover, dry, and fix it over a spirit lamp or Bunsen's gas burner until it commences to give off vapour; then float the cover, sputum downwards, upon the surface of a small quantity of fluid (1) in a staining saucer; leave it for fifteen minutes.

In the case of sections, immerse them in the fluid for twenty minutes.

Now decolourise in 1 part of sulphuric acid to 7 parts of distilled water, until all colour has disappeared. Wash away all trace of the acid in distilled water, when a slight tinge of colour will become perceptible. Float the cover upon, or immerse the sections for a few minutes in, solution 2. Wash in distilled water, place in absolute alcohol for three or four minutes. Let the cover become perfectly dry, and mount in xylol balsam. In the case of sections dehydrate them in absolute alcohol, clear them in xylol and phenol, and mount them in xylol balsam. (The xylol in which the balsam is dissolved will clear the sputum on the cover.)

2. Dr. Heneage Gibbes' Formula.

R Magenta Crystals, 2 grammes.

Pure Anilin, 3 grammes.

Absolute Alcohol, 20 c.c.

Distilled Water, 20 c.c. 
Float a thin glass cover upon which sputum has been spread or immerse sections, as in the former process, in this fluid, for twenty minutes.

Decolorise in a 33 per cent. solution of nitric acid in distilled water, wash free from acid and float the cover upon, or immerse the sections for a few minutes in, a saturated solution of chrysoidin in distilled water; when they are stained brown, wash them in distilled water, place them in absolute alcohol for a short time. Clear the sections in xylol and phenol, and mount in xylol balsam.

If a small crystal of thymol is dissolved in absolute alcohol and added to each of the solutions in the above two formulæ it will tend to their preservation. Each of these solutions should be filtered into the staining saucers when used.

\section{Ehrlich's Process.}

Make a saturated solution of phenylamin in distilled water. This is done by prolonged shaking up the water with the floating anilin and filtering; add to the filtered mixture a saturated solution of fuchsin in absolute alcohol until a brilliant red colour is obtained. Now, as in the former processes, spread a thin layer of sputum upon a thin glass cover and dry, and fix it over a spirit lamp or Bunsen's gas burner. Float the cover upon the fuchsin solution for from fifteen to twenty minutes, wash it in distilled water and float it upon a 33 per cent. solution of nitric acid in distilled water for a few seconds, when it will be found quite colourless. Now float the cover for a few minutes, on solution No. 2, of formula No. 1. Wash in distilled water, dehydrate in absolute alcohol, mount in xylol balsam. 


\section{Weigert's Process for Schizomycetes. Bacilli, Bacteria and Micrococci.}

$\mathbf{B}_{\boldsymbol{x}}$ A 2 per cent. solution of Gentian Violet in Distilled Water, 12 c.c., and a Saturated Aqueous Solution of Anilin oil (filtered) 100 c.c.

Stain the sections in this.

Make a Solution of Vesuvin, 1 gramme.

Rectified Alcohol, sp. gr. 830, 10 c.c.

Distilled Water, 100 c.c.

Stain in this for fifteen minutes.

Clear in xylol and phenol.

Mount in xylol balsam.

Rev. Gordon Thompson's Process.

The special feature of this valuable process consists in fixing the bacilli, bacteria or micrococci, after staining them with the ground colour, by subjecting them to the action of a solution of gold made by dissolving 15 grains of gold chloride in 4 ounces of distilled water. This solution is to be poured upon the cover, on which the bacilli have been spread and allowed to remain upon it for two or three seconds and then poured off again, the cover is then to be washed in distilled water. The gold appears to form a tube round the bacteria and so encased, they appear to be rendered absolutely permanent; preparations, in the author's possession mounted by this process twelve years ago, remaining as brilliant in colour as the day they were made.

For the ground stains-

Methyl violet, fuchsin and acid green are used, the covers being floated-upon the solution, washed in distilled water, then treated with the gold solution, and again washed in distilled water. 
For the second stains use-

Acid green, Bismarck brown or chrysoidin; wash in distilled water.

Mount in pure glycerine jelly containing no trace of acid.

The best contrasts of colour will be found to be-

Fuchsin followed by acid green.

Fuchsin followed by anilin (or soluble) blue.

Bismarck brown followed by anilin (or soluble) blue.

Methyl violet followed by acid green.

\section{Clearing Media.}

Specimens which are to be mounted in Canada balsam or any other resinous media must be penetrated by a "clearing" agent, which shall render them perfectly transparent and remove from them all traces of the alcohol in which they have been dehydrated or kept. It is now generally admitted that hardened balsam, which has been re-dissolved in xylol is the best and most reliable preparation of that most useful, valuable and permanent mounting medium.

It is obvious that a clearing agent which consists in great measure of the solvent of the balsam must be peculiarly adapted to precede the balsam, and it may at once be said that the best, the cleanest in its application, and the easiest to use, consists of a mixture of exactly equal parts of xylol and absolute phenol. The manner of its use is simplicity itself, whilst its penetrative and "clearing" actions are practically instantaneous. Much has been said and written about elaborate processes for running the 
clearing agent under the sections, letting them sink through alcohol into the clearing agent placed at the bottom of a test tube or saucer, with alcohol above it, raising the sections with "lifters," \&c., but this complicated process tends to the destruction of the sections, which cannot be too little manipulated. Now, in xylol and phenol we have a medium which requires no such treatment, all that is necessary being to place the fluid in a saucer to the depth of about a quarter of an inch. The saucer should be a large one, so that there shall be no difficulty in removing the sections, or any necessity for dragging them up the side of the saucer. Place the sections for five minutes in absolute alcohol, transfer them, with a thick curved needle which has a blunt point, to the surface of the clearing fluid. The alcohol is almost instantly removed and the sections sink to the bottom. Then take a perfectly clean cover, and with the curved needle float a section carefully on to the cover and spread it perfectly flat, drain the excess of the fluid on to a piece of blotting paper, by holding the cover in forceps and placing its edge upon the blotting paper, then lay the cover section upwards upon the blotting paper to remove the clearing fluid from its under surface, and before the clearing fluid evaporates apply xylol balsam to the section. The balsam being miscible with the clearing fluid, there is nothing antagonistic or greasy to remove, which is the case when oil or turpentine, or both, are employed as clearing agents.

This medium is the best that can be used for clearing sections cut from specimens which have been embedded in celloiain, upon which it has no solvent effect. 
When, however, it is not desired to mount with the section its surrounding and supporting celloidin, Turpentine will be found the best solvent of that material as well as of paraffin when that has been used as the embedding agent.

Turpentine should not be used for specimens or sections which have been hardened or preserved in alcohol or methylated spirit, as it has great shrinking power. It will be found very useful for entomological preparations, and especially for insects with very hard integuments; these must be left to soak in turpentine until they are cleared, but small and delicate insects can be better cleared in xylol and phenol. Turpentine is also a good clearing agent for vegetable specimens of a woody nature. All specimens or sections cleared by turpentine should be mounted in Canada balsam, hardened and redissolved in turpentine, and carefully filtered.

Oil of Cloves has some advantages over all other clearing agents (xylol and phenol excepted), and is, perhaps, the most universally used. It combines well with both alcohol and balsam and does not shrink the specimens as turpentine does; but "clearing" with this oil involves its removal by placing the sections in turpentine after they have been cleared, because if the clove oil remains in the specimens or sections, although it is miscible with the balsam, it prevents the balsam from hardening and so renders the preparations unsafe.

Sections cut from specimens which have been embedded in celloidin and which it is desired to mount so embedded must not be cleared in oil of cloves, as it dissolves the celloidin at once; it is therefore a good clearing agent when it is desired to 
remove celloidin. It must be remembered that oil of cloves renders specimens which have been allowed to remain in it very brittle.

Oil of Bergamot is a valuable, though costly agent; it clears the celloidin embedded specimens and does not affect anilin stains to any great extent. It is fairly rapid in its action and clears well.

Cedar Wood Oil.-This oil is not expensive, but is very slow in its action, it also has the valuable properties of clearing perfectly, however slowly, and of not injuriously affecting anilin dyes, or of shrinking specimens which have been hardened or preserved in alcohol. It is very useful for clearing vegetable sections, though extremely slow in doing so, and celloidin sections may be cleared in it, but it takes several hours to render them transparent.

\section{Mounting Media and Cements.}

Many media in which specimens may be examined and delicate dissections made for examination, are not in the true sense of the term preservative and therefore serve only a temporary purpose. Many solutions again which re-act upon tissues, and elucidate their features and composition serve only, in like manner, for purposes of temporary examination and study. It is intended, in the following list of media in which specimens and sections can be mounted, to give the formulæ and methods of using and applying the best preservative media so as to secure the permanence and safety of the preparations which are made with them. The list will be supplemented with formulæ for the most useful fluids in which to make dissections and to place 
tissues or specimens for temporary observation and examination only.

Canada Balsam.-The most universally used and useful of all mounting media is the oleo-resin obtained from Abies Balsamea and Pinus Canadensis and is mostly imported from Quebec. The balsam is obtained from the tree by puncturing the blisters, or vesicles, which form under the bark of the trunk or its branches, and collecting their fluid-contents in a bottle. It is at first opaque, but gradually clarifies and becomes transparent. It consists of 24 per cent. of volatile oil, 60 per cent. of resins soluble in boiling alcohol, and of 16 per cent. of resins soluble in ether, but insoluble in alcohol.

As it hardens very slowly, it is, for histological purposes and for use as a mounting medium, generally evaporated to dryness and re-dissolved in either benzol or xylol, preferably in the latter.

These solutions harden rapidly, are perfectly transparent, practically permanent and preservative. For some exceptional purposes, e.g., for specimens which require to be mounted in deep cells, pure, undiluted balsam will be found useful.

In order to prepare Canada Balsam for histological purposes it should be gently heated over a sand bath and thus slowly evaporated until it becomes quite hard. Great care must be taken that the heat does not become too great so that the balsam is burned, in which case it becomes of a dark brown colour and is consequently spoiled. When quite dry and brittle it is to be reduced to a coarse powder and re-dissolved in an equal quantity of one or other of the solvents above named. For delicate entomological and some vegetable prepara- 
tions hardened balsam may be re-dissolved in turpentine-all these solutions of balsam, whether in xylol, benzol or turpentine must be carefully filtered for use, and should be kept in wide-mouthed capped bottles.

\section{Glycerine Jelly.}

R. Glycerine (Price's), 50 c.c.

Distilled Water, 42 c.c.

${ }^{1}$ Boracic Acid (Crystals), 2 grammes.

Gelatine (Gold label), 6 grammes.

1 new laid Egg.

Soak the gelatine in water for at least twelve hours. Pour off the water, and place it in a vessel with the distilled water. Melt it in a water bath. When melted, add the glycerine and acid. Remove the vessel from the water bath, beat up the white (only) of the egg, and thoroughly incorporate it with the fluid at a temperature of from $30^{\circ}$ to $35^{\circ} \mathrm{C}$. (not more). Replace the mixture in the water bath, and gently boil it until it has assumed a flaky appearance. Now filter it through the finest filtering paper, which must be done either in a warm oven, or with a hot filter. Keep it in a well stoppered stock bottle, and place some, for use, in a wide mouthed capped bottle. When used, the bottle should be placed in a bath of water sufficiently heated, and kept so, to thoroughly liquify the "Jelly."

1 Carbolic Acid (Crystals), or Formic Acid may be substituted for this ingredient-the former, indeed, is generally used-but Boracic Acid does not_affect hæmatoxylin stainings, which are unquestionably sooner or later much injured by the other acids. 


\section{Lawrence's Formula.}

Soak $\frac{1}{4} \mathrm{lb}$ of best gelatine for some hours in cold water, pour off the water, and heat the gelatine until it melts. Let it cool, but whilst it still remains liquid, add to each (fluid) ounce a (fluid) drachm of white of egg. Boil gently until it becomes clear, filter it through fine flannel, and add to each ounce of the solution 6 drachms of a mixture of 1 part (Price's) Glycerine and 2 parts of Camphor water.

\section{Kaiser's Formula.}

Place one part of finest "gold label" French gelatine in 6 parts of distilled water, let it soak for two or three hours, then add 7 parts of best glycerine, and for every 100 grammes of this mixture, L gramme of strong Carbolic acid. Warm for from ten to fifteen minutes, stirring all the time; when all the flakiness has been removed, filter it.

\section{Gelatine and Honey. (Deane's medium).}

R Gelatine (Best " gold label "), 1 ounce.

Honey, 5 ounces.

Distilled water, 5 ounces.

Rectified alcohol, $\frac{1}{2}$ ounce.

Creasote, 6 minims.

Soak the gelatine in the water until it is quite softened. Boil the honey in a water bath, and when it is boiling add the gelatine and water. Now boil the mixture, let it cool slightly, then mix the alchohol and creasote together and add them. Filter through fine flannel. 


\section{Gum and Glycerine. (Farrant's medium). ${ }^{1}$}

1.-Gum arabic (Best picked), 4 ounces.

Distilled water, 4 ounces.

Glycerine, 2 ounces.

Keep it in a well stoppered bottle with a lump of camphor in it.

2.-Gum arabic, 1 ounce. $^{2}$

Glycerine, 1 ounce.

Distilled water, 1 ounce.

Arsenious acid, $1 \frac{1}{2}$ grains.

Dissolve the arsenious acid in the water, and the gum in this mixture, without heat, add the glycerine and incorporate with great care to avoid the formation of air-bubbles.

Glycerine, mixed with distilled water, forms an admirable medium in which to examine tissues and specimens and make minute dissections. It is sometimes used thus diluted as a mounting fluid, but is not to be relied upon as a preservative medium, whereas undiluted glycerine, when once the crucial difficulty of sealing it up and preventing leakage from the preparation has been successfully achieved, may be considered not only unsurpassable as a mounting fluid in cases for which it is suitable, but entirely reliable as a preservative. The hermetically sealing of the glycerine under the cover, or in the cell, must be most carefully done by applying at least three coats of shellac cement to be followed by two or three coats of white zinc cement, the formulæ for making which will be given in their places, and the method of applying them

${ }^{1}$ Beale, page 68. $\quad{ }^{2}$ Micrographic Dictionary. 
described in the chapter on Mounting. All tissues, sections, and specimens, to be mounted in pure glycerine, should be subjected to a prolonged immersion in glycerine and distilled water, the proportion of the glycerine to that of the water being gradually increased every day or two until pure has been substituted for diluted glycerine. Great care must be taken that the specimen shall be thoroughly saturated with the glycerine before mounting it therein.

This soaking may be done either in watch-glasses or very small staining saucers, and many specimens or sections can thus be immersed together. In order to prevent the glycerine from absorbing rnoisture from the atmosphere, and to ensure cleanliness, the saucers should be placed under bellglasses whilst the soaking is in progress. This process, moreover, prevents the serious shrinkage which too frequently results, especially with delicate tissues and specimens, which have been mounted in glycerine without previous saturation therewith. Tissues, sections, delicate marine animals, insects, $\& c .$, which are to be mounted in glycerine may be preserved therein until required.

Glycerine undoubtedly affects logwood stainings adversely, but if slightly acidulated with acetic acid it does not attack ${ }^{1}$ carmine or picro-carmine.

Acetate of Potash.--Make a saturated solution in distilled water, it may be so used or diluted if

${ }^{1}$ Farrant's medium is preferable for the mounting of specimens stained by either of these. It is advisable to rather overstain and to soak the specimens in the medium for two or three days, in order to extract the superfluous stain before mounting them. 
necessary. This fluid should be allowed to flow under the cover by capillary attraction, and the mount be sealed up in from twenty-four to thirtysix hours afterwards.

\section{Naptha and Creasote. (Dr. Beale's formula.)}

Creasote, 3 drachms.

Wood-naptha, 6 ounces.

Distilled Water, 64 ounces.

Chalk, quant. suff.

Mix the naptha and creasote together, and add sufficient chalk to produce a thick paste. When this has been mixed smooth, add gradually the water, thoroughly incorporating all the ingredients in a mortar, and let it stand in a lightly covered vessel for two or three weeks. Stir it occasionally. Then pour off the super-natant fluid, which, if necessary, filter. Finally, add some small lumps of camphor, and keep it in a well-stoppered bottle.

\section{Goadby's Fluid.}

Bay Salt, 4 ounces.

Alum (powdered), 2 ounces.

Corrosive Sublimate, 4 ounces.

Distilled Water, 2 quarts.

This is an admirable preservative for delicate marine specimens, all entomostraca and allied organisms; for larvæ and some entomological specimens.

Mounting Fluid for Algæ (of which it preserves the colour) and for fresh gatherings of diatomaceæ, desmidiaceæ, \&c.

Acetate of Copper, 15 grains.

Camphor Water, 4 ounces.

Distilled Water, 4 ounces.

Acetic Acid (glacial), 20 minims. 
Mix and add

Glycerine (Price's best), 8 ounces.

Bichloride of Mercury, 1 grain.

Mix thoroughly, and filter very carefully,

Styrax.-A liquid gum which exudes from the bark of the Liquidambar orientale. This is used specially for the mounting of diatomaceæ, and the method of its use will be described when, in the chapter on "Mounting," full instructions are given for cleaning and mounting these organisms. Styrax is soluble in alcohol, ether, benzol, and xylol, which last is its best solvent for microscopical purposes. The solution should not be too thick, and must be carefully filtered. Styrax is preferable to Canada Balsam as a medium in which to mount diatoms, its refractive index being greatly higher than that of Balsam, whilst it is equally safe and durable.

Monobromide of Naphthalin is soluble in alcohol and ether; its high refractive index-double that of Canada balsam-renders it a valuable mounting medium for diatomaceæ. The cover should be "ringed" with wax before any cement is applied to secure it. Then two coats of shellac varnish, followed by white zinc cement, or, if the slides are to be used with oil immersion objectives, by the special cement of which the formula is given amongst the

\section{CEMENTS.}

Dammar Varnish.-Gum dammar, dissolved in benzol, has been much used as a mounting medium; its only recommendation, however, is that it is easy to apply. It is not safe, neither is it durable, and the author strenuously advises that 
it shall be entirely discarded for all purposes of mounting, and used only as a cement, in which character it has its uses, and especially it is good for " ringing" glycerine jelly and glycerine "mounts" before the application of the shellac varnish, and the finishing white zinc cement,

Dammar varnish is made thus :-

$$
\begin{aligned}
& \text { R Indian Gum Dammar, } 4 \text { drachms. } \\
& \text { Benzol (best) } 8 \text { " (fluid). }
\end{aligned}
$$

Let it stand for two or three days, then pour off the super-natant liquid, and add thereto 1 drachm of spirits of turpentine. Filter if necessary.

Bell's Cement. ${ }^{1}$-This is a thoroughly reliable cement for many purposes; it is easy to use, as it flows readily from the brush and hardens rapidly. Shallow cells can be made with it, and it may be used for sealing up preparations mounted in fluids, glycerine, or glycerine jelly, in place of shellac varnish, the cover being previously " ringed" with the following :-

\section{Gelatine Cement. (Marsh's formula.)}

Soak half an ounce of gelatine in water until it is thoroughly soft. Pour off nearly all the water, and melt the gelatine in a hot water bath; add 3 or 4 minims of creasote and keep it in a capped bottle. It must be used warm, it sets very quickly, and may be followed by Bell's cement, or shellac varnish, and the slides be finished with white zinc cement.

1 This cement must be purchased from its makers, Messrs. J. Bell \& Co., 338, Oxford Street, London, or from the opticians; its formula being a secret. 
Asphalt Varnish.-It is better to purchase this also. Mr. Kitton, however, finds that asphalt dissolved in benzol, and to which is added some gold size, answers its purpose well.

Gold Size.-This, again, should be purchased, as also should Marine GIue, which, as well as the following cement, is used for affixing cells to slips.

\section{Gotta Percha Cement. (Harting's formula.)}

Divide gutta percha into small pieces, and dissolve it, by means of moderate heat, in 15 parts of oil of turpentine; when dissolved, strain it through linen, and whilst the solution is kept at a gentle heat, stir into it one part of shellac.

For affixing cells to the slips, heat the cement, make a ring on the slip the size of the cell, attach the cell and heat the slip until the cell is affixed by an even layer of the cement, so that no leakage may occur.

Shellac Cement or Varnish. - Break up shellac into very small flakes, and put these into a bottle with strong methylated spirit; shake it up frequently, until there results a solution sufficiently thick to be used easily with a brush. When from keeping it becomes too thick, add more spirit.

\section{White Zinc Cement.}

(Walmsley's formula), and given by him to the author.

In order to ensure that the white oxide of zinc - which is the foundation of this valuable and permanent cement-shall be of the best quality, and well ground, it is advisable to purchase a large tube of artist's "zine white," rather than to grind for 
oneself, the ordinary white oxide of zinc in drying oil. The tube of zinc white is to be emptied into a large bottle, and as much as possible of the oil to be removed, by shaking up the white zinc in a considerable quantity of benzol, and when the zinc has settled to the bottom of the bottle, pouring it off. A saturated solution of gum dammar in benzol is to be made in another bottle, which should be placed in a water bath, kept hot, until the gum dammar is dissolved. When this is accomplished the solution of gum dammar is to be poured upon the white zinc, the bottle thoroughly shaken up, and the mixture strained through fine muslin. Now add about half a drachm of best gold size to each ounce of the solution. If too thin, allow the benzol to evaporate until the cement becomes of a consistency to flow smoothly and readily from the brush-if too thick, add benzol. This cement should be kept for use in a capped bottle with a wide mouth, and thoroughly stirred (not merely shaken) up each time it is used. White zinc cement properly made according to this formula, is thoroughly reliable and safe. It never cracks, will stand ordinary wear and tear well, but not, of course, rough usage. The author's experience is, that no other white zinc cement than this is of any value or permanency. Preparations intended for use with oil immersion objectives must not be finished with this cement, as the essential oils used for immersion purposes at once attack and soon destroy it.

Cement for finishing preparations to be examined with immersion objectives.

Place five or six pieces of gum mastic, about the 
size of a pea, in sufficient strong methylated spirit to dissolve them thoroughly.

Soften some isinglass in water, remove all the water and dissolve it in two ounces of absolute alcohol.

To this add two small pieces of gum ammoniacum rubbing them up in a mortar until they are dissolved.

Mix the two solutions, and keep the cement in a wide mouthed stoppered bottle.

When using it place the bottle in hot water until the cement is liquefied.

\section{Embedding and Freezing.}

The great desideratum in embedding specimens, for the purpose of making sections of them, is, that they shall be firmly and equably supported in all directions and that no distortion, displacement or injury to the tissues, organs or organisms embedded shall result. It is therefore most important that the student should acquire the knowledge and experience necessary to enable him to decide which process will afford and ensure the delicacy combined with solidity required in special cases.

The methods of embedding are twofold, " simple" and " interstitial," the former consisting in enclosing the specimen to be sectionised in a soft substance which will consistently and equably support it in all directions; whilst the latter involves the careful infiltration of the specimen with, and its thorough permeation by, a "mass" which, whilst warm, is liquid, and which solidifies as it cools; or, as in the case of celloidin, the saturation of the specimen with that substance, held in solution by ether, 
which evaporating leaves the specimen perfectly embedded in a transparent and firmly gelatinous matrix.

Lee, in his invaluable Text-Book ${ }^{1}$ treats of the "theory of embedding" in a manner so exhaustive and practical that the author ventures to quote largely from his clever pages and tendering his best acknowledgments. Lee, then, says, after referring to the simplest methods of embedding: "A further object is proposed in the case of the other (i.e., the more elaborate) class of methods, which may be designated methods of interstitial embedding or infiltration methods. In these it is proposed to fill out with the imbedding mass the natural cavities of the object, in order that their lining membranes, or other structures contained in them, may be duly cut in sit $\hat{\imath}$, or, going a step further, it is proposed to surround with the supporting mass not only each individual organ, or part of any organ that may be present in the interior of the object, but each separate cell, or other anatomical element, thus giving to the tissues a consistency they could not otherwise possess, and ensuring that in the thin slices cut from the mass all the details of structure will precisely retain their natural relations of position. Such a process of embedding is at the same time practically a process of hardening, in so far as it enables us to give to tissues a degree of firmness that could otherwise only be obtained by the employment of chemical processes, such as prolonged treatment with chromic acid and the like. The

1 "The Microtomist's Vade Mecum" (page 162). J. \& A. Churchill. 
principle of the methods of this second class is either, (like that of the first,) that by the immersion of the object to be cut in some material that is liquid whilst warm, and solid when cold, all the parts of the object may be duly surrounded by the supporting mass (the second class differing from the first chiefly in the employment of materials possessing greater power of penetration whilst liquid, in longer immersion in the liquid mass, and in such previous preparation of the object, by soaking in some liquid that is a solvent of the embedding material, as makes it more readily susceptible of infiltration by the latter); or the processes may be based on another principle, namely, that of the employment of substances which whilst in solution are sufficiently fluid to penetrate the object to be embedded, whilst at the same time after the evaporation or removal by other means of their solvent, they acquire and impart to the embedded object sufficient firmness for the purpose of cutting. The collodion process sufficiently exemplifies this principle. If a piece of soft tissue be dehydrated, and soaked first in ether and then in collodion, and if the ether be allowed slowly to evaporate, the tissue and surrounding mass of collodion will acquire a consistency such as to admit of thin sections being cut from them."

Embedding in Carrot. - This is a very primitive and almost exploded process, but it has its uses and advantages when cylindrical specimens, for instance, vegetable stems, are to be embedded; it is also available for such tissues as can be rolled up and so enclosed in the centre of the carrot. A "well" microtome is, of course, neces- 
sary to this process, whilst a set of cork cutters, or of brass tubes sharpened at one end, must be provided; one of these tubes must be of the exact diameter of the well of the microtome, and the others, progressively, of smaller diameters, so that the various diameters of the specimens may be matched. A sound carrot must be chosen and a cylinder cut out of its centre, with the largest tube, precisely to fit the well of the microtome, and of the same length as the depth of the well; now, with a knife divide this cylinder into two equal halves, place the two halves together, and with the tube nearest in size to, but slightly smaller than, the specimen to be embedded cut out the exact centre of the cylinder; this will give two semi-circular pieces of carrot each with a semicircular groove, in one of which place the specimen, lay the other piece upon it, and the specimen is embedded in a cylinder fitting tightly into the well of the microtome, and sections can be cut, after practice, of a great degree of thinness, and without difficulty.

Embedding in Pith.-This substance is used in the same manner as the carrot. A cylinder of pith exactly the size of the well of the microtome is divided longitudinally into two equal halves, and the specimens embedded between the two halves, and if possible without the removal of any of the central pith. Pressure and "kneading" will generally be found sufficient to form a central tube, in which the specimen can be enclosed, with the advantage that no portion of the pith having been actually removed, the cells displaced by pressure will to a great extent recover their original position and exert the desired central pressure and give 
the necessary " all round" support to the specimen. The embedding being accomplished, the cylinder is to be driven into the well of the microtome and moistened with alcohol, which will cause the pith cells to swell and firmly enclose and support the specimen, whilst if the cylinder should not already fit into the well with the necessary exactitude this swelling will ensure perfect tightness.

Wax and Oil.-Equal parts of white wax and olive oil are to be melted by heat over a sand bath. In warm weather, or in a hot room, the proportion of wax should be slightly increased; in colder weather, or in a cool room, increase the proportion of oil. The mass must, of course, be melted when used. The specimens to be embedded should be immersed in alcohol and thoroughly dehydrated, and then placed for a few seconds in collodion, which being allowed to evaporate to dryness, the specimen is then embedded either in the well of the microtome or in a paper tray. The ring of collodion surrounding the sections will be dissolved away by the clearing medium.

Embedding in Paraffin. - The infiltration with paraffin, and subsequent embedding of specimens therein, is a process of much nicety and requiring great care, and first, the selection of a suitable paraffin and the composition of the "mass" are all-important. Two paraffins with the different melting-points of $110^{\circ} \mathrm{F}$. and $140^{\circ} \mathrm{F}$. should be combined in such proportions as shall give a firm and homogeneous mass which, when after melting it has solidified, will cut perfectly smoothly in a temperature of from $55^{\circ}$ to $70^{\circ} \mathrm{F}$. It will be found in practice that two parts of the paraffin of the $140^{\circ}$ 
melting point, and one part of that melting at $110^{\circ}$, will afford a mass which will answer perfectly well when cut in a temperature of $70^{\circ}$. That with the lower melting point being combined in the proportion of one-fifth of its bulk with four-fifths of that of the higher melting point, will cut well in a temperature of $60^{\circ}$; whilst equal parts of the two paraffins at $110^{\circ}$ and $140^{\circ}$ melting points respectively will give the most satisfactory results when cut in a temperature of $55^{\circ} \mathrm{F}$.

The paraffin is to be melted, in a water oven, in porcelain pipkins (having covers and handles), and the embedding may be done in paper or cardboard trays, but it will be found more convenient and much safer to use, according to the size of the specimen to be embedded, the small porcelain pans in which moist water colours are sold (these give nice little firm blocks requiring no trimming); for larger specimens, small porcelain vessels used in various chemical processes are readily procurable, whilst small glass vessels, or large (flat-bottomed) test-tubes will answer admirably for specimens of any considerable size.

The specimens must be thoroughly hardened and perfectly dehydrated, and when about to be embedded are to be placed in strong alcohol, or, better still, in absolute alcohol, for a short time; from this they are removed into creasote and left therein until they are well impregnated (say for six hours). Having removed as far as possible all creasote from their surfaces, the specimens are placed in the melted paraffin, in which, kept at a temperature slightly above its melting-point, they are to remain from one to four hours according to their size, and to 
ensure absolute permeation by, and saturation with, the paraffin. The blocks must be allowed to cool thoroughly before they are removed from the moulds; when removed, they may be pared down to any size desired, and in such a manner that the face of the embedded specimen, from which it is intended to cut sections, shall be at the surface. A sufficient body of paraffin must be left surrounding the specimen to ensure the necessary solidity and equable support. Specimens thus embedded and prepared for sectionising may be kept for an indefinite time without alcohol, and used when required. As they are perfectly embalmed and preserved, the only necessary precaution is to ensure absolute freedom from moisture in the bottles or boxes in which the blocks are stored.

The paraffin can be dissolved out of the sections by means of chloroform, turpentine, turpentine and oil of cloves mixed in equal proportions (this will also clear the sections), or xylol; the latter being of course preferable when the sections are to be "cleared" in xylol and absolute phenol, and mounted in xylol-balsam. When turpentine (only) is used, and the sections are to be cleared in xylol and phenol, they must be placed in absolute alcohol for a short time to remove the turpentine, before being immersed in the clearing fluid.

Specimens can also be satisfactorily embedded in paraffin by the following method (Giesbrecht's), founded upon the rapid evaporation of chloroform, and which, at the same time, is a speedy solvent of paraffin. The specimens to be embedded having been immersed to saturation in absolute alcohol are next placed in chloroform; a short time having 
been allowed for saturation therewith, the chloroform and the specimens are together heated to the melting point of the paraffin, small pieces of paraffin being gradually added during the heating; air bubbles will arise from the specimens, and when these cease no further addition of paraffin will be necessary. The "mass" must be kept at its melting-point in a dry oven for some considerable time after the addition of the paraffin, in order to ensure the entire evaporation of the chloroform, any remains of which in the mass would prevent its becoming sufficiently hard to cut satisfactorily. The embedding is of course done as in the ordinary paraffin process.

Embedding in Gum and Glycerine.

R Gum Acaciæ Mucilage, B.P., 90 parts.

Price's Glycerine

10 "

Add 1 per cent. of carbolic acid crystals.

Place the specimens to be embedded in this mixture in watch glasses, or small (water colour) saucers, and let them remain exposed to the air of the laboratory for four days. In that time the mixture will have set into a firm, gelatinous mass. Blocks, in each of which one specimen is enclosed, are then to be cut out, and again left exposed for three days to harden, being turned over from time to time that they may dry equally on all sides. When dry, the blocks may be pared down to suitable size, and affixed to corks with strong gum water, and on these they may be preserved, to be sectionised as required.

The gum can be removed from the sections by immersion in distilled water before staining them. 
Embedding in Celloidin. ${ }^{1}$ - This delicate and cleanly and most effective process is pursued as follows :

A thin solution of the celloidin is made in equal parts of absolute alcohol and ether.

A thick solution is also made in the same manner, by increasing the proportion of the celloidin.

The specimens-perfectly dehydrated - are to be placed in absolute alcohol for twenty-four hours, and then transferred into a mixture of equal parts of absolute alcohol and ether for a second period of twenty-four hours.

They are now to be immersed in the thin solution of celloidin for twenty-four hours, and afterwards in the thicker solution for three days.

They are then to be placed in paper trays, so made as to admit of a good margin of celloidin surrounding the specimen occupying the centre of the tray. The tray is then to be filled with the thick solution of celloidin, which is to be allowed to evaporate, the tray being filled up from time to time, as evaporation goes on, until the specimen is covered, as well as surrounded, by a layer of celloidin-in other words, embedded in a block of celloidin, of the size and shape of the tray. When the celloidin begins to set firmly, and before any shrinkage sets in, the tray, with the specimen, is to be plunged into a vessel with an air-tight cover, containing strong methylated spirit, and in this the

1 " Schering's Celloidin," which is patented, can be obtained at Mr. C. Baker's, 244, High Holborn, London, W.C. The celloidin shavings, recently introduced, will be found much more convenient to use than the tablets. 
blocks may be preserved until required for sectionising. The purpose, in embedding in celloidin, is to secure the normal position (in situ) of all the component parts of an organism or specimen. The film of celloidin surrounding all the organs and parts, and preserving their respective features and positions, must be retained, and mounted with the section. When these sections are to be mounted in Canada balsam, they must be cleared in xylol and phenol (as oil of cloves destroys the celloidin) and mounted in xylol balsam. Celloidin sections may be mounted in glycerine, glycerine jelly, or Farrant's medium, all of which render the celloidin nearly as transparent as does balsam. These sections may be stained by means of all the ordinary staining fluids, except, perhaps, one or two of the more powerful anilin dyes which sometimes, though curiously enough not invariably, colour the celloidin indelibly. If stained by logwood they should be strongly stained in order to admit of the extraction of the colour from the celloidin by the acetic acid process, whilst the tissues remain sufficiently stained.

Specimens embedded in celloidin should be cut by the ether freezing process.

Gum and Syrup.-For ordinary tissues make a mixture of 5 parts of gum mucilage B.P. and 3 parts of simple syrup B.P. For nerve, brain, spinal cord, and any brittle tissues the combination should be 5 parts of gum mucilage to 4 parts of syrup.

Tissues and specimens to be cut upon the ether freezing microtome are to be immersed in this fluid for three or four days. They may be preserved for 
an indefinite time in the mixture, indeed, permanently. The specimens must have been carefully hardened and thoroughly freed, by prolonged soaking in water, from all traces of the hardening reagents, before placing them in the gum and syrup.

The ether freezing process is conducted thus. The gum and syrup is wiped off the specimens with a cloth, and they are placed in the strongest possible solution of gum acaciæ for a few hours before being frozen and cut. The microtome and freezing apparatus having been prepared for use, a specimen is placed upon the freezing plate and painted round the edges and over the surface by means of a brush with the strong, pure gum-water from which it was taken. It is then frozen by means of the ether spray until it can be easily and smoothly cut. Practice and experience will soon enable the operator to stop the freezing at the right moment. Should the ice, however, become too hard, and therefore brittle, a few seconds' delay before commencing to cut the sections will ensure its being in the right condition for cutting.

\section{On Section Cutting and Microtomes.}

Section cutting, like staining, is an art requiring not only practice and experience, but knowledge and judgment. Thorough knowledge of the structure and functions of the organisms, organs and specimens to be sectionised is equally necessary with the judgment enabling one to decide as to the thickness or thinness of the sections of, say, an organ which shall most satisfactorily display its structure. It is quite possible to cut sections too 
thin - so thin, indeed, as to obliterate all the features and structure which it is necessary to study in order to arrive at the physiological or biological elements of the specimen. Brain, spinal cord, and the like tissues, for example, cannot be cut too thin-kidney and several other organs can easily be so, and thus be rendered worthless for purposes of study. It is well, therefore, to provide one's self with three sections of such specimens, as it may be found possible, by experience, to cut too thin; so that for general observation and study, with low powers, a thin section is at hand, for medium powers a thinner section, and the thinnest for use with high and the highest powers.

Some writers and practical workers assert that it is not possible to cut the thinnest sections from a celloidin-embedded specimen by the ether freezing process; the author can only say that he has cut sections of entire human eyes, so embedded and frozen, 5000ths of an inch thick (or rather thin), and of brain and spinal cord, \&c., $\frac{1}{500}$ th of an inch thin.

For cutting sections by the ether freezing process there is (with the exception of the automatic Minot microtome recently produced and fully described in later pages) no better microtome than the Cathcart, as perfected and supplied by Mr. Alexander Fraser, of Edinburgh (which is fully described and illustrated on pages 127-8), with admirable arrangements and apparatus for embedding specimens in paraffin and for cutting those also, the freezing and this arrangement being removable at will and interchangeable. 


\section{Professor Rutherford's Microtome.}

This is Stirling's original well and screwmicrotome, plus an ice box arrangement. It is used by filling the ice box with well-powdered and mixed ice and bay salt in equal quantities. Whilst freezing is going forward the well may be covered with felt. One or several tissues can be cut at once, either with a Rutherford's knife, or, what is now found to be far better, a plane iron. By a plane iron, we mean the iron taken out of a carpenter's smoothing plane. These irons must be $2 \frac{3}{4}$

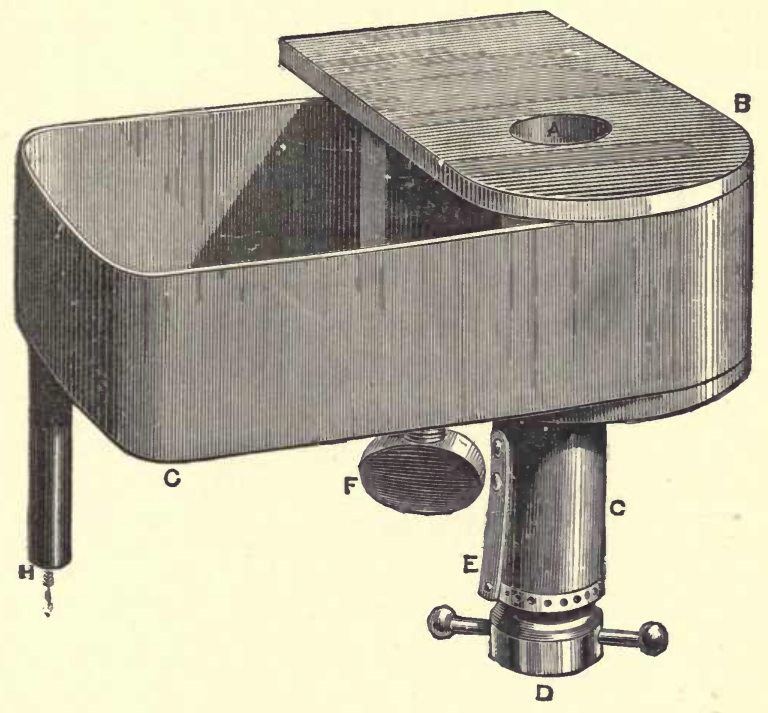

inches broad, and are used either with or without a covering of wood. When double-faced with wood, the handling of them is more pleasant, but it does not add to efficiency. The iron must be sharpened on a very smooth hone, and then well stropped. In sharpening, care must be taken to rub the entire 
length of the cutting edge evenly. This is best done by taking care to rub the edge on the stone in such a way that every portion of its length is upon the stone at the same time. In stropping, it will be found most convenient to rest the plane iron against a table edge, and push or pull the strop over it.

The advantage of the Rutherford freezing microtome is that it can be also used as an ordinary Stirling's microtome for imbedding in carrot, paraffin or wax. Its disadvantage is the same as that of

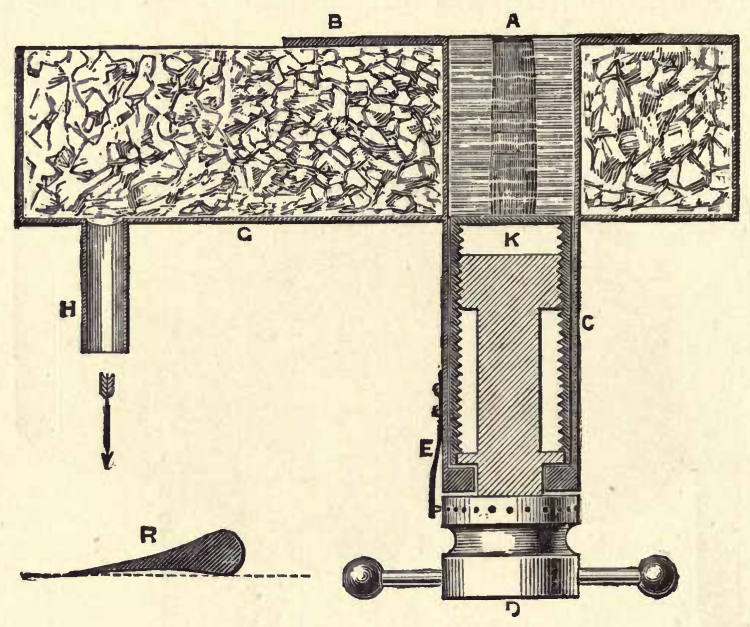

ice freezing machines of whatever description-that it is cold, disagreeable work filling in the ice and salt, and the former is not always to be obtained, and when once charged, cutting must follow, or the ice melts.

Mr. C. Baker, 244, High Holborn, W.C., sells an excellent Rutherford's freezing microtome, which, of course, must have its cutting face covered with plate glass. 


\section{Williams' Freezing Microtome.}

This is made by Messrs. Swift \& Son, 81, Tottenham Court Road, London, W.C., who also make a registered knife carrier, which carries the blade of a razor: the whole goes under the name of "Swift's Knife." Until the plane iron was thought of, Swift's Knife had the field all to itself, and there will still be those who will prefer it to the plane iron.

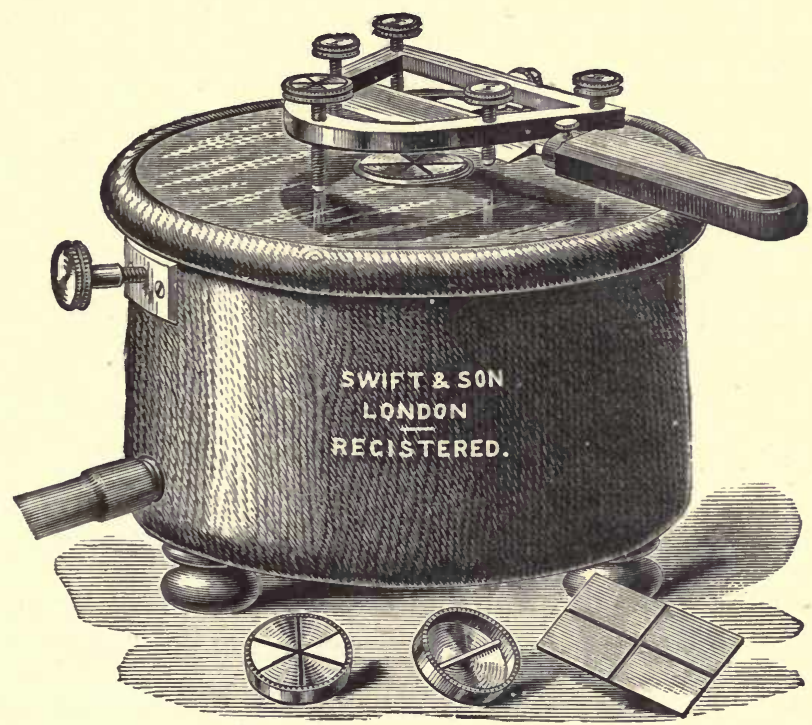

This combination has this great advantage : that in winter, when ice is plentiful, the machine is charged, and freezes strongly for an hour or more. For class work this is excellent: the attendant charges the machine just before the class meets, and the demonstrator cuts a hundred sections of any prepared tissue in a few minutes. If he wishes to cut another specimen, he removes the first, and 
clears out the grooves on the top of the section carrier with the back of a knife ; then he places his specimen upon the machine, and paints it round with gum solution, and the whole freezes fit for cutting in one minute or so. With very little practice it is quite easy to cut fifty excellent sections of each of fifteen or twenty specimens with one charge of ice and salt.

To manage Swift's knife, we proceed thus :First take the blade away, and lay it flat upon a smooth hone well oiled, and rub it first on one side, then the other, until the entire edge on either side is ground by the hone at once. The writer rubbed over twenty hours before this was accomplished with the razor blade of his Swift's knife. Both sides of the blade are ground quite flat, to begin with, not hollowed; but cutlers either cannot or will not grind a blade with its back and its edge perfectly parallel, so that this has to be done by one's self. Having ground the knife blade, we fix it in the handle and strop it. We then take off the handle and fix the blade in the carrier in such a way that the edge must be perfectly level with the top of the microtome, and on a much lower level than the back. This position of the knife in the frame is to the last degree essential, and we effect it thus:-First place the knife-blade in the frame, and lower the screws which the edge of the blade rests upon, so that the edge is lower than the back a good deal. After making it firm, we complete the levelling by freezing a piece of tissue, and taking away a thick section, cutting away from us, with the knife in the frame jüst as we have put it roughly. We now place each end of the blade alternately 
against the same part or bit of the frozen tissue, and raise or lower either end by screwing either of the two screws nearest to us. All is now complete, and we cut section after section by lowering the knife edge for each fresh cut by giving the screw furthest away from us a part of a revolution.

\section{To Fill an Ice Microtome.}

Get a piece, or pieces, of ice the size of two closed fists, and the same bulk of either common table salt or, even better, of bay salt. Powder both, and thoroughly mix them. The ice is most readily reduced to fine powder by being surrounded with flannel and bruised with a broad-faced hammer, or a carpenter's mallet. Fill in the mixed ice and salt and press it well down, but take care that the trough of the microtome is not so full that the top touches the ice and salt. The top is to be placed on the microtome and screwed fast. In winter the machine, after being charged, is ready for freezing in five minutes or so; in summer it may be ten minutes or more. Freezing proceeds, as we have before said, for an hour or two. The waste pipe at the bottom of the microtome is to be connected by caoutchouc tubing, with a basin to receive the melted ice.

The disadvantage of Williams' microtome is, that, like all ice microtomes, it is disagreeable to charge even when ice is plentiful, and cutting must take place at the time the machine is charged; that is to say, if the operator is called away, as in medical practice often happens, his ice charge melts and has to be renewed. 


\section{Dr. Fearnley's Ether Freezing Microtome.}

Dr. Groves suggested the conversion of Williams' ice freezing microtome into an ether freezer. This is now called the Grove-Williams microtome, and is

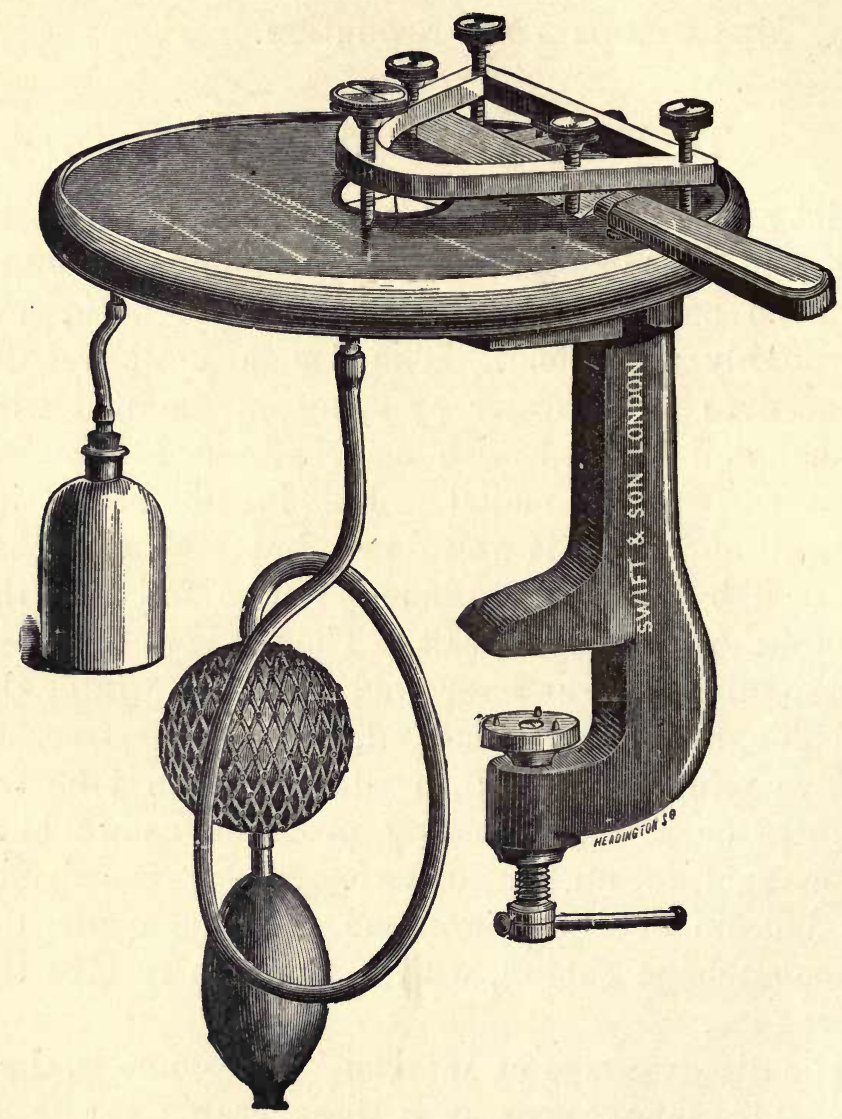

made by Messrs. Swift \& Son, and used with Swift's knife.

For those who are not affected by the inhalation of atmospheric air charged with ether, the best and simplest ether freezer for use with Swift's knife is 
Dr. Fearnley's ether freezing microtome. This is simply the top of the Grove-Williams' microtome, fitted with a clamp and screw arrangement for attaching it to the work-bench. The ether nozzle is immediately under the frame of the glass plate, and the bottle of ether stands under the machine on the table as shown in wood-cut. Fearnley's microtome is also made by Messrs. Swift \& Son.

\section{Cathcart's Microtome.}

This is an ether freezing microtome for use either with a stout razor, or with a plane iron. Cathcart's invention, used with a plane iron, for cheapness, simplicity, and efficacy cannot be surpassed, if equalled, by any ether or ice microtome we know of. With the expenditure of two drachms of ether, the operator can cut sixty or seventy sections in almost as many seconds, every section being of exquisite thinness. The ether used is methylated ether of 720 s.g.

The freezing process has already been described. This being accomplished, the tissue is elevated to the knife by a very small fraction of a revolution of the screw with the left hand, whilst the right drives the plane iron. The iron must be held with the edge far below the level of the rest of the iron, and the screw movement and the push of the iron movement must take place alternately. When a mass of sections has accumulated on the iron, it must be floated off into a saucer of water. (See illustration on page 128.)

An admirable and most useful microtome is made by Zeiss. It can be seen at the depôt of, and purchased from, his London agent, Mr. C. Baker. 
The "Rocking Microtome" manufactured and supplied by the Cambridge Scientific Instrument Company, is in all respects an admirable and most

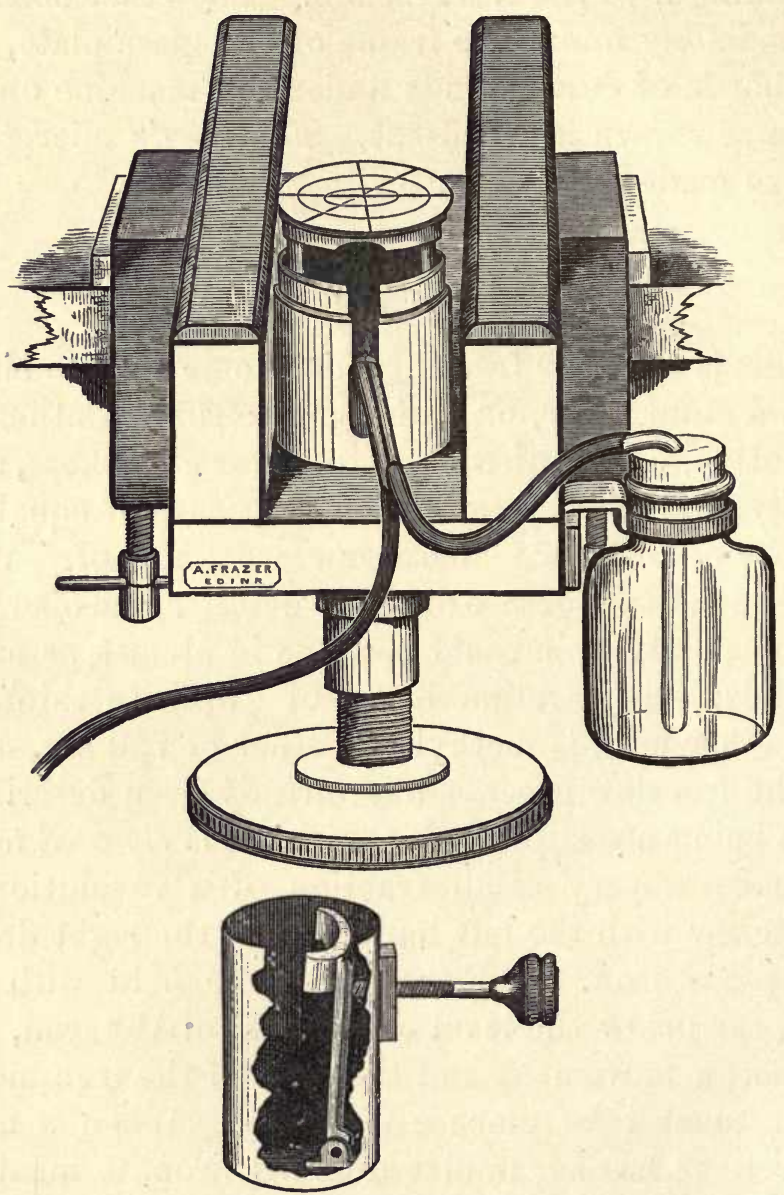

The Cathcart Microtome.

effective machine, and especially for the cutting of serial (or ribbon) sections. A full illustrated description, as well as directions for its use, can be obtained on application to its makers. 
The "Thoma Microtome" adapted equally for the paraffin embedding and ether freezing processes, is an exquisitely beautiful and reliable instrument. The preferable size for ordinary use is that named the Thoma B. 2. A verbal description would be of but little use, and the author advises the student to see one, and this he can (generally) do on application to Mr. C. Baker. Its manufacturer, Mr. R. Jung Mekaniker, Heidelberg, will send a full (illustrated) description on application.

\section{Minot's Microtome.}

This beautiful instrument is made in three forms (or to use the inventor's expression, "models").

In its construction the chief endeavour has been to render its various parts as "massive" as possible, and to make the adjustments for the various thicknesses of the sections perfectly automatic, whilst, at the same time, portability has been considered. The thickness of the section is regulated by a special toothed wheel arrangement. On the ascent of a vertical slide a lever strikes one of the six spokes of a wheel, and the ratchet turns the toothed wheel of the micrometer screw so that the horizontal slide, to which the preparation is attached, is brought into contact with the edge of the knife. The six spokes of the controlling wheel are of different lengths, and are marked 1 to 6 , which numbers correspond with the number of teeth on the wheel moved at each revolution of the driver.

In Model I. the total number of teeth is 150 , and as one complete turn of the micrometer screw equals 0.5 m.m., by means of the spoked wheel sections 
can be obtained of the respective thicknesses of $\frac{1}{300}, \frac{1}{150}, \frac{1}{100}, \frac{1}{75}, \frac{1}{80}$, and $\frac{1}{50}$ th $\mathrm{mm}$., or of from about 3 to 20 mikrons.

In Model II. thinner sections are obtained by means of a differential gear. A second wheel is used, and the various spokes, 1 to 6 , give sections of from 1 to 6 mikrons.

In Model III. the fine adjustment is again modified by the use of two ratchets engaging in the toothed wheel, one of which is a half tooth shorter

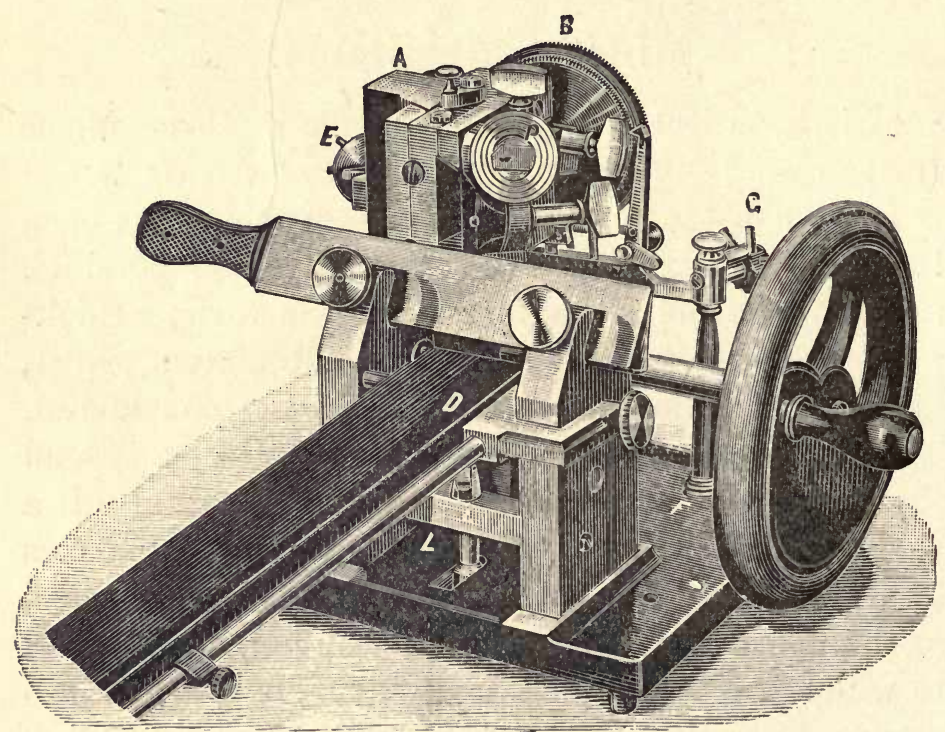

than the other. The co-operation of this shorter ratchet allows further tenuities of section of from $\frac{1}{2}$ to $1 \frac{1}{2}$ mikrons.

- The advantages and improvements secured by the Minot microtome are, briefly, as follow :-

1. The practically indestructible solidity of all its parts. 
2. The smooth and even motions of its slides.

3. Its moderate size and weight and corresponding portability.

4. The great range of section thicknesses, from $\frac{1}{2}$ to $40 \mathrm{~mm}$.

5. Its applicability to large sections up to $5 \times 5$ cm.

6. The forward movement of the specimen entirely above the knife.

7. The easy adjustment of the specimen in every direction.

8. The production of absolutely flat and even sections, \&c., \&c.

Model III. ${ }^{1}$ can be used for cutting specimens embedded by the celloidin process, for the ether freezing process and for "immersed" cutting.

\section{Vegetable Tissues}

and specimens can be preserved for an indefinite time and for sectionising in 1 part glycerine, 1 part alcohol, and 1 part water. This, however, applies to such specimens as are not readily obtainable, and which therefore must be kept in stock. When practicable these tissues should be gathered and cut, by the freezing process, whilst quite fresh, as in this way the best results will be secured and all distortion or shrinkage avoided.

1 This new microtome also can be obtained from Mr. C. Baker, 244, High Holborn, London, W.C. 
CHAPTER VI.

\section{On Mounting.}

UNTIL within the last few years it was considered de rigueur to mount all preparations upon the ordinary sized slips (3 in. by 1 in.) and the author having ventured to produce slides of large sections mounted upon correspondingly wide slips, was told by one of our most accomplished microscopists and workers that if he (the author) persisted in, when necessary, increasing the size of his slips, "everyone would curse him." That curse, like most others, has fallen very harmless, and now we have in England, as has long been the case on the Continent, preparations of large size mounted upon slips and covered with the thinnest glass covers of dimensions corresponding with the size of the section or other specimen to be mounted. As a rule, however, the ordinary sized slip is adhered to, and save in exceptional cases, and under special necessities or circumstances, this is for several reasons, and especially because the arrangements of the mechanical stage of the microscope are made for slides of those dimensions, advisable and convenient.

Thin cover glasses are to be procured cut into 
circles, squares, oblongs and ovals and of any required size; circular or oval covers will be found the most convenient to use, and the neatest in appearance; circular covers can be "ringed" by the aid of the turn table with any of the various protective cements. Cover glasses are supplied of three thicknesses, known respectively as No. 1, No. 2, and No. 3. No. 3 covers are to be avoided as too thick. No. 2 will be found quite thin enough for all ordinary purposes. No. 1 must be used for all preparations intended for the higher powers.

An ounce of No. 1 covers should have a fair proportion of $\cdot 003$ inch glasses in it, still more of $\cdot 004$. than $\cdot 005$, advancing in proportion from $\cdot 003$ to $\cdot 006$. Should an ounce of No. 1 covers contain more than a dozen, or at most a score, over $\cdot 006$, it ought to be rejected. As the objectives now used are oil immersion, or ought to be, for powers higher than an eighth the thickness of the cover may be of less importance. For instance, Messrs. Powell and Lealand's $\frac{1}{12}$ oil immersion lens works through a cover of 006 inch perfectly. Almost anything in histological work can be seen with an objective of a $\frac{1}{6}$ inch power: only exceptionally do we require so high a power as the $\frac{1}{12}$ inch. We ought, however, to use no cover thicker than a 006 inch, if possible. There are numerous cover-glass measures. Messrs. Ross make an excellent one.

\section{Mounting in Canada Balsam.}

Many years ago the author discovered the following method of using Canada balsam, and having made no secret of it, it is generally known 
as Cole's exposure process. It consists in placing the specimen to be mounted upon a cover glass, applying exactly the quantity of balsam necessary to ensure the permanent preservation of the specimen, and that the balsam shall flow to, but not beyond the extreme edge of the cover, and then allowing the balsam to evaporate for twenty-four or thirty-six hours until all the xylol, or other solvent, has left it, and the balsam has set hard before mounting it on the slip. The author devised a set of "pigeon holes," or. rather grooves, made to take $3 \mathrm{in}$. by 1 in. slips, in which the covers could be placed, lying upon slips, during the time allowed for evaporation. But when the small cabinets, with trays, were introduced, he found these to be, practically, all that is needed for this purpose. This process of mounting is thus effected. A supply of thin glass covers of the sizes necessary for the specimens to be mounted having been cleaned, and the specimens, if necessary, cleared, they are placed upon the covers-if sections, being floated on to the covers from the clearing fluid and carefully spread flat upon the covers. The clearing fluid is then drained off by holding the cover with its edge against a sheet of blotting-paper - and the covers, if $\frac{3}{4}$ in. diameter, are placed in pairs upon slips. If $\frac{5}{8}$ in. covers are used, three are placed on each slip, of course with the section, or other specimen, upwards. The balsam is then applied, and the slips with the covers, balsam rpwards, placed in a cabinet with trays so that no dust can approach them, and they are left for twenty-four hours to evaporate. Then a very small drop of xylol balsam is placed in the centre of each of the mounts in succession; a 
slip is heated and the covers are successively turned over on to the slips and the balsam is carefully " run" by gentle heat until a neat, bevelled edge surrounds the cover. The balsam will be found quite hard, when cold, and, if necessary, the cover can be washed with spirit and any excess of balsam removed from its edge and its surface cleaned. Never mount specimens or sections in balsam on the slip, placing the cover on the liquid balsam and trusting to time for its drying. Practically the central portion of the balsam, under the cover, never dries, and no such slides are really safe or permanent.

When specimens are mounted in cells filled with balsam, Xylol-balsam should be used and allowed to evaporate before the cover is applied to the cell, the cell being filled up from time to time as evaporation goes on. In this way specimens can be mounted in cells in hard balsam and rendered absolutely permanent.

\section{Mounting in Glycerine.}

Place the slip on a turn table, and run upon it a ring of asphalt. The ring should be the size of the cover, which, of course, should be a circle. When the asphalt is half dry, but still "tacky," place the section on the slip, and put upon it a drop of glycerine; then lower the cover over it in the usual way, and gently press the edge of the cover all round, so as to make it adhere to the asphalt. Should it not do so at any point, gently warm the asphalt by holding the slip over the flame of a spirit lamp; then press the cover home. The slide can 
now be dipped in water, and any glycerine that has overflowed or been pressed out, washed off with a camel-hair brush. After drying it can be ringed with shellac varnish followed by white zinc cement.

\section{Mounting in Glycerine Jelly.}

Specimens to be mounted in glycerine jelly should be immersed in distilled water for some time before, in order to ensure the removal of all alcohol from the tissues, \&c. This being done, place a widemouthed bottle containing glycerine jelly in a water bath kept warm, but not too hot, by a spirit lamp or a Bunsen's gas burner placed beneath it. Now place the section or other specimen to be mounted precisely in the centre of the slip, and apply the jelly with a small pipette. Then holding a clean cover of the necessary size in forceps, steadily and gently lower it upon the jelly. The cover should touch the slip with its edge furthest from the operator and be gradually lowered so as to drive out air bubbles, if any, and not to disturb the position of the specimen. This is a delicate operation, requiring great care and some practice and experience. The secret of success is-do not be sparing of your jelly, apply plenty and you will not be troubled with air bubbles. When cold the excess of jelly can be easily removed with a soft brush, and the slide should be allowed to dry (not rubbed dry) and then ringed with shellac cement and finished with two coats of white zinc cement.

\section{Mounting in Farrant's Medium.}

The author has constantly been asked how it is that air bubbles are entirely absent from all pre- 
parations mounted by him in Farrant's medium, and has listened sympathetically to the sorrows and disappointments of those who find "the difficulties of mounting in Farrant's medium insuperable;" he has also read of a clumsy and absurd arrangement for passing this medium through a funnel stuffed with cotton wool, which is supposed to eliminate the air whilst ensuring the agreeable addition of cotton fibres to the slide in place of air bubbles. $\mathrm{He}$ can only say that he has had great practice with and experience in the use of Farrant's medium during many years, and has found no difficulty whatever in getting rid of air bubbles. Here again he advises-put an abundant supply, with a glass rod, on to the slip, place the specimen, which should be immersed in Farrant's medium for twenty-four hours before mounting it, in the centre of the drop. If there are any large air bubbles, as there possibly may be, explode them with a red hot needle or push them to the margin of the drop, where they will disappear at once. If there are any large air bubbles in the specimen, remove these also with a needle; small bubbles will disappear by absorption within a day or two. Finally, do not press down the cover too much at first so as to cause too great an exudation, from under it, of the medium; leave it resting lightly upon the specimen for a few days during which all bubbles, if any, will go, and the medium will "set" to some extent; then press down the cover, and the medium having so "set" will not again be drawn under the cover by capillary attraction to anything like the extent that it is when first applied; and this pressing down of the cover can be repeated several times at intervals of 
days, and at length the excess of the medium can be washed off with a soft brush, the slide, as in the case of the glycerine jelly, be allowed to dry, ringed with shellac varnish, and finished with white zinc or asphalt.

Specimens to be mounted in aqueous media, such as carbolised water, camphor water, glycerine and water, \&c., should be immersed for some hours in distilled water, and mounted, from that, in a shallow cell as already described in the case of glycerine.

\section{The Preparation and Mounting of Insects.}

'There are several processes for preparing and mounting insects, each of which possesses special advantages, in respect of the results and appearances it may be desired to obtain. Whenever it is possible, and except for special purposes and in dissections, insects should be mounted "without pressure," since the flattening them out under pressure results in distortions, displacement of the parts and organs, and unnatural, and therefore false appearances: It is, however, impossible to mount all insects, or their parts, without pressure, and the following process will give good results :-

Place the insect in pure liq. potass. mixed with $\frac{1}{5}$ ammonia fort. The insect must not be allowed to remain too long in this solution, and must be tested from time to time by placing it in water and pressing the thorax. When the thorax is soft and the legs flaccid, immerse the insect in water for from fifteen to twenty-four hours, then soak it in glacial acetic acid and glycerine (half and half) for some 
hours. ${ }^{1}$ Again, immerse it in water for from twelve to twenty-four hours; it is now ready to be laid out upon the slip. Having done this, preserving the natural position of the parts as closely as possible, place a cover over the insect and tie lightly round it with soft cotton, stand the slip on end to drain, plunge the slip into a turpentine bath and leave it until all moisture has been driven out and the insect is thoroughly permeated by the turpentine, drain off the turpentine, apply blotting paper to remove any excess of turpentine from under the cover, and run in "xylol balsam" by capillary attraction. The cover should never be lifted or allowed to shift its position after the insect has been laid out.

When it is desired to preserve the brilliant or delicate colours of an insect, it should be placed, immediately after being killed by means of chloroform, in liq. potass. without any mixture of ammonia, for from two to three days, then soaked in water for twenty-four hours, then placed in water with ten drops of muriatic acid to each ounce of water, for twenty-four hours; it should then be laid out and immersed in turpentine and treated as already described.

In order to mount insects, or their parts, " without pressure," it is generally necessary to adopt only the following simple process :- Soak the insect for two days in equal parts of ordinary alcohol and water, after which transfer it to absolute alcohol for

1 Insects removed from liq. potass., after being soaked in water for twenty-four hours, should be kept in strong or glacial acetic acid and glycerine (half and half) until required for mounting. 
two days, immerse it in turpentine until it is not only completely permeated therewith and all air removed, but let it remain until it is sufficiently bleached or decolourised-in other words, rendered transparent-and in order to ensure this result it should be placed in a strong light. Select a cell of the requisite depth, of pure tin or vulcanite, which affix to the centre of a slip with the French liquid glue; when this is dry, having cleaned the interior of the cell, place within it the insect, and fill the cell with fairly thick balsam until it presents a slightly convex surface above the cell, lay it aside, as previously recommended, under cover for twelve to twenty-four hours, so that all air may escape, put a minute drop of fresh balsam upon the surface of the balsam which fills the cell, and carefully place a cover, slightly warmed, upon it, close the cell by gentle and equable pressure, at once remove, by means of a soft brush and benzol, all balsam which has exuded under the pressure, let the "mount" harden for a day or two, and then apply white zinc cement or asphalt.

Cells are to be obtained of various sizes, having pure tin caps to fit exactly over them after the cover is applied, and these not only impart great strength and security to the cell, but ensure the neatest possible finish to the mount. Mr. F. Enock, whose exquisite entomological preparations are altogether unrivalled, is to be credited with this admirable device, as well as with various other improvements in the special branch of the art to which he has devoted himself with such singular success. Insects may be mounted "without pressure" in suitable cells filled with pure glycerine 
instead of balsam, and for such preparations Mr. Enock's capped cells will be found invaluable. Insects mounted in glycerine must, of course, be previously prepared by dehydration, in spirit, after the potass. process, and subsequent prolonged immersion in glycerine until complete saturation results.

\section{The Preparation of Vegetable Sections.}

Whensoever possible, stems, leaves, roots, petioles and wood should be cut fresh and sectionised as soon as may be; all such specimens should be kept in a mixture of equal volumes of alcohol, glycerine, and water. Sections also may be stored in this mixture. Nearly all vegetable sections require bleaching before being stained; very delicate tissues may be bleached by means of alcohol; hard and deeply-coloured stems and woods must be bleached in a liquid thus prepared :-

To one pint of water add two ounces of fresh chloride of lime; shake this up thoroughly two or three times, and allow it to stand until the lime shall have settled. Make a saturated solution of common "washing soda." Pour off the supernatant fluid from the chloride of lime, and, by degrees, add to it the soda solution, until all precipitation ceases. Filter the solution, and keep it in a stoppered bottle in the dark. No fixed time can be given for the bleaching process, the colour and density of tissues being so variable. Experience, however, will be rapidly gained, and over-bleaching easily avoided. The sections being bleached, must now be washed in distilled water, several times changed, and allowed 
to remain for twenty-four hours in the final water, to which add 8 or 10 drops of nitric acid to each half pint. Transfer the sections to alcohol for an hour before staining them. To bring out details of structure and to display cell walls, \&c., there is no better stain than logwood, and for singly stained vegetable sections it is to be recommended in preference to all other staining fluids. The following process will give admirable results :-

1. Remove the section from alcohol to water for a few minutes.

2. To 3 per cent. alum solution for ten minutes.

3. Stain in aqueous logwood stain.

4. Place in alum water to remove stain from surfaces.

5 . Wash thoroughly in water.

6. Dehydrate in strong alcohol.

7. Clear in xylol and phenol.

8. Mount in xylol balsam.

\section{The Double Staining and Mounting of Botanical Sections.}

1. Bleach the sections for six hours in the liquid already described.

2. Soak them in water, to be several times changed, for twenty-four hours.

3. Place them, for twelve hours, in the following:

Mordant Solution.

(a) Sulphate of Aluminia, 10 grains.

Dissolved in Distilled Water 200 grains.

(b) Acetate of Lead, 30 grains.

Dissolved in Distilled Water, 600 grains.

Add $a$ to $b$ until precipitation ceases, then allow the whole to settle and syphon off the clear liquor, 
which must be filtered and kept in a stoppered bottle. When required for use dilute a portion with four or five additional equivalents of water and filter afresh.

4. Stain in borax carmine solution for one to two hours.

5. Wash in acidulated water (1 drachm nitric acid to 1 pint of water).

6. Wash quickly in pure water.

7. Place in alcohol for one hour.

8. Place in green stain for one to three hours.

Acid Green, 2 grains.

Distilled Water, 3 ounces.

Glycerine, 1 ounce.

Mix the glycerine and water well together and dissolve the green crystals in the mixture, filter and keep in a stoppered bottle.

9. Wash quickly in absolute alcohol.

10. Clear in xylol and phenol.

11. Mount in xylol balsam.

\section{Another Process for Vegetable Sections.}

1. Place the section in an alcoholic solution of iodine green ( 3 grains to the 1 ounce of alcohol) for one or two hours.

2. Soak it in alcohol for ten minutes.

3. Remove it to water for one minute.

4. Immerse it for two hours in carmine fluid, made as follows :-

Carmine, 15 grains.

Ammonia, 15 grains.

Water, 2 ounces.

Dissolve the carmine in the ammonia by means of gentle heat, add the water, and filter. 
5. Wash thoroughly in water.

6. Place in alcohol for ten minutes. balsam.

7. Clear in xylol and phenol, mount in xylol

\section{Picro Carmine, Double Staining.}

Picro Carmine as a selective double stain cannot be surpassed. The process is as follows :-Take of carmine 2 grains, liquor ammonia $\frac{1}{2}$ drachm, distilled water 1 ounce; dissolve the carmine in the ammonia by means of gentle heat, add the water. Dissolve 8 grains of picric acid in 1 ounce of alcohol, also by means of gentle heat, and mix the two solutions.

Place the sections in alcohol for one hour; immerse them in the recently filtered staining solution for from half an hour to three hours, i.e., until they are sufficiently stained; wash them in alcohol, immerse them in an alcoholic solution of picrate of ammonia for one hour, and for a second hour in a like solution; in other words, change the solution once during the two hours. Place them in alcohol for a few minutes, clear in xylol and phenol as already described, and mount in xylol balsam.

Unstained vegetable specimens may be mounted in balsam, glycerine jelly, or Farrant's medium.

\section{The Preparation of Diatomacea.}

Diatoms may be said to be absolutely ubiquitous; recent and fossil, living or dead, they are readily obtainable in vast numbers, and of infinite variety. In nearly all waters, fresh or salt, still or running, 
in marshy districts, on the surface of the ocean, or attached to algæ and shells, they abound in a living and growing state, whilst their siliceous valves, after the death of the cell-contents, are to be found, in a more or less perfect condition, as deposits at the bottom of rivers and ponds and on the sea floor. From the stomachs of holothurians, ascidians, molluscs, and of various shell-fish, exquisite varieties have been obtained in profusion. As fossils they form beds of enormous extent, intermixed with the siliceous casts of polycystina, radiolarians, \&c., and the calcareous shells of foraminifera, whilst fossil deposits purely diatomaceous and yielding the most beautiful, rare and varied forms have been discovered in all parts of our globe. In order to clean diatoms and render them fit for mounting, as either "selected" or "strewed" slides, considerable experience and great care are necessary. The subsiliceous varieties especially require very delicate treatment, whilst with many of the fossil and recent forms, which are intensely siliceous, altogether " heroic" and drastic measures must be resorted to in order to ensure perfect cleansing of the surfaces of the valves. There have been nearly as many nostra propounded and published for the cleaning and mounting of diatoms as there are genera and species of the diatomaceæ themselves. Years of experience and constant experiments have led the author to the conclusion that the simplest and safest modes of treatment are the best. For living diatoms-and especially for the sub-siliceous forms (e.g., pleurosigma) - the following method will give perfect results :-Remove all dirt, débris, and salt, by repeated washings, thoroughly shaking up the 
gathering each time and allowing the diatoms to settle before pouring off the water; in this way all soluble impurities can be removed. When the water remains clear, after shaking, pour it off, leaving the diatoms as nearly as possible dry; cover them to about one inch in depth with absolute alcohol, or the strongest ordinary alcohol, which will gradually dissolve and extract all the endochrome from the valves; change the alcohol daily until it ceases to be tinged with green. Wash away all trace of alcohol, pour off all water, place the diatoms in a platinum capsule and heat them to a dull red over a "Bunsen" burner; this will separate the frustules into single valves, and complete the cleaning of the diatoms, which may be now bottled up in distilled water for future mounting.

\section{To clean Diatoms growing upon Algæ or} Shells. - Place the algæ or shell débris in a large basin (in order to allow space for effervescence), cover them with water, add gradually, hydrochloric acid, and stir until violent effervescence results; add acid little by little, until effervescence ceases -thoroughly stirring from time to time-strain through net of sufficiently fine texture to retain the débris whilst the diatoms pass through it. When the forms shall have thoroughly settled down, pour off all water and place the strained deposit in a large test-tube. Boil in pure hydrochloric acid for twenty minutes; add pure nitric acid, and boil again for twenty minutes. Whilst boiling add crystals of chlorate of potash until complete bleaching results ; remove all trace of acids and alkali by repeated washings, and examine the forms; if perfectly clean bottle them up for mounting; if, as is sometimes 
the case, there has been animal matter present, which has not been entirely removed, boil in pure sulphuric acid for a few minutes, and wash away all trace of the acid before placing the diatoms in distilled water, for keeping.

\section{To clean Fossil Diatomaceous Deposits. -} Some fossil deposits are of such extreme hardness that it is necessary to resort to caustic potash for their disintegration; it is, however, better, whensoever possible, to dispense with this powerful alkali, since, even in the most careful and practised hands, more or less abrasion of the surface of the valves will generally be found to result from its use. When a fossil deposit will not yield to milder treatment, it must be broken into small pieces, placed in a test-tube, just covered with the solution of caustic-potash, and boiled for half a minute (only) over the flame of a "Bunsen" burner; pour off into a glass beaker nearly full of water all the deposit which has been disintegrated by the potash and repeat the process until all the lumps are broken up. Then, when all the forms have settled to the bottom of the water in the beaker, pour off the water, remove the diatoms into a large test-tube, fill the test-tube nearly full of water, add a small quantity of bicarbonate of soda, and let the diatoms gently "simmer" in this weak solution for two hours. Wash away all soda, and boil the diatoms for a few minutes in pure nitric acid. Remove all trace of acid by repeated washings, and keep the diatoms in distilled water.

Fossil deposits which can be disintegrated without caustic potash should be boiled in a test-tube of large capacity, in a moderately strong solution of 
bicarbonate of soda-the disintegrated portions being, from time to time, poured off into a beaker and the boiling in bicarbonate of soda repeated until all the deposit has broken up, when the alkaline solution should be washed away and the diatoms boiled for a short time in nitric acid-all traces of which are to be removed by repeated washings and the forms kept in distilled water for future use.

To mount Diatoms "dry."-All "dry" mounts of diatoms, whether "strewed" or "selected," are liable to destruction or deterioration from an accumulation of moisture upon the under side of the cover, which moisture, sooner or later, and in defiance of all precautions, always makes its appearance. "Dry mounts" are therefore always more or less unsatisfactory and unreliable and to be avoided as much as possible. The best method of mounting diatoms "dry" whether for "test" or as "arranged" slides-is to make a cell of the best asphalt, of the necessary thickness, by adding coat upon coat of the asphalt, not by making the cell of sufficient depth at one operation. Spread the diatoms upon the cover, if necessary " burn" them upon the cover, i.e., place the cover upon a piece of thick platinum foil and raise it, slowly and carefully, to a dull red heat over the flame of a "Bunsen" burner; thoroughly heat the slip with the asphalt cell upon it; whilst it is hot (and therefore certainly free from all damp or moisture) place the, equally hot, cover carefully upon the cell, pressing down the cover and making sure that it adheres thoroughly and evenly to the cell all round-run a ring of asphalt round the edge 
of the cover; when this is hard, "ring" the cell with two coats of white zinc cement, letting the first coat dry thoroughly before applying the second.

\section{To mount Diatoms in Canada Balsam,} \&c.-Canada balsam has been, hitherto, universally employed as the best medium in which to mount diatoms. It has stood the test of time, and proved fairly permanent and reliable. For the mounting of diatoms, however, hardened balsam re-dissolved in benzol or xylol is by no means so satisfactory in its results as ordinary balsam, since it does not admit of being submitted to the application of direct heat, after the slide is mounted; any attempt so to harden the balsam resulting in the production of air-bubbles, which carry away the forms in the ebullition caused by the endeavour to drive out the air, and without heat, applied directly, the balsam never "sets" hard. Diatoms should therefore be mounted in chemically pure, filtered balsam, diluted to the consistency of cream with turpentine. The diatoms in "strewed" slides should be allowed to fall upon the cover from a "pipette" held at a height of three or four inches above it-the sudden fall of the drop causing the diatoms to spread evenly upon the surface of the cover. ${ }^{1}$ The drop upon the cover should then be dried, very slowly, by means of a "Bunsen" burner placed underneath the brass table upon which the cover (lying upon a 3 in. by $1 \mathrm{in}$. slip) has been placed. When it is desired to mount many slides of the same gathering of diatoms

1 The even spreading of the diatoms on the cover will be further ensured by breathing upon it before allowing the drop to fall, whilst in selecting diatoms, if the cover upon which they are to be placed is breathed upon, the forms will adhere to it. 
the requisite number of covers should be cleaned, and three of them laid upon each slip, previously breathed upon to cause the covers to rest firmly upon it, and the slips laid upon the brass table; a drop of water containing the diatoms should then be allowed to fall upon each cover, as previously recommended, and the covers can all be dried together; a sufficient quantity of the turpentinebalsam should then be put upon the covers, which should be placed in a cabinet with trays, or under a glass shade, so that all dust may be excluded, for twenty-four hours. By this means the absolute permeation of the forms by the balsam, and the consequent removal of all air are secured before the final completion of the mount, an advantage so obvious that further comment is unnecessary. The covers should then be replaced upon the brass table and subjected to gentle heat from a "Bunsen" burner until the balsam is hard; the cover should then be taken up by means of forceps, and a slip being made hot the cover should be placed precisely in the centre of the slip, ${ }^{1}$ which should then be held over the "Bunsen" burner until the balsam runs to a neatly bevelled edge round the cover. No airbubbles will trouble the mounter if this process is carefully practised. This is the so-called "exposure-

I In order to ensure the placing the cover exactly in the centre of the slip, either of the three following devices will be found convenient:-

Firstly. Upon a small white tile draw, in china painting colours (which should be burnt in), a 3 in. by 1 in. space (representing a slip), with its precise centre strongly marked. This tile will be found of great use in suddenly cooling balsam, when that may be desirable.

Secondly. Cover a 3 in. by 1 in. slip with white paper, rule 
process," which was invented by the author in 1870 , and the success of which induced him to devise the necessary modifications upon it and to construct the necessary appliances in order to adapt it to the mounting of histological and other preparations. Slides of "selected" diatoms should be mounted in the same manner, the forms being picked up by means of a carefully chosen hair from the dried hide of a cow-the soft and flexible hairs taken from the under side of the neck being those most suitable for the purpose. These hairs are to be affixed to the cedar sticks used with "camel-hair pencils" by means of sealing-wax dissolved in alcohol, and a number should be prepared, having the hairs of different lengths in order to obtain varieties of strength and elasticity. Some hairs should be mounted of considerable length, as the mounter of diatoms and other such minute specimens must learn to put them back symmetrically into their required positions under the microscope, and in the balsam when they float away; a little practice will soon lead to the acquirement of this absolutely necessary skill, and it will be found not difficult to heat the hardened balsam (many times if necessary),

lines faintly from each top corner to its opposite bottom corner; the intersection of these lines will give the exact centre of the slip, which can be marked by a dark spot.

Thirdly. With a writing-diamond make, upon a slip, placed on a turn-table, concentric circles, $\frac{1}{2}$ in., $\frac{5}{8}$ in., $\frac{3}{4}$ in., and $\frac{7}{8}$ in. in diameter; each sized cover can then be placed precisely in the centre, if the slip on which the cover is to be placed is laid upon that upon which the circles have been described, the circles, of course, being clearly visible throngh the superimposed slip. 
and whilst it is hot to, very rapidly, replace a form in its desired position, whilst with a fine needle all hairs and other imperfections can be removed and absolute cleanliness ensured.

About a quarter of a century since, when slides of "selected" and "grouped" diatoms, \&c., were in the zenith of their popularity, and finding, from painful experience and repeated failures, that the successful mounting of these and other such preparations was surrounded by almost insuperable difficulties, in respect both of affixing the siliceous valves to the thin covers on which they were to be placed, and the selecting perfect forms from the débris and refuse of the cleaned diatomaceous material, and safely transferring them to the slide in course of preparation, the author, in order to conquer these difficulties, devised a cement (for which the formula will be given in its place), and which has proved equally safe, permanent and invisible, even under the highest powers of the microscope, and, indeed, the only really reliable method of fixing diatomaceæ, spiculæ, polycystina, \&c. It is the only cement, also, so far as the author knows, which has stood the test of many years, all other cements used for this purpose (and all of which he has tried) having sooner or later, and generally sooner rather than later, succumbed to the disintegrating action of the Canada balsam, or other media in which the forms have been mounted, with the result that they have become detached from the cover and valuable and expensive slides thus destroyed and rendered worthless. Finding also by equally annoying experience that the so-called "mechanical finger" and other such 



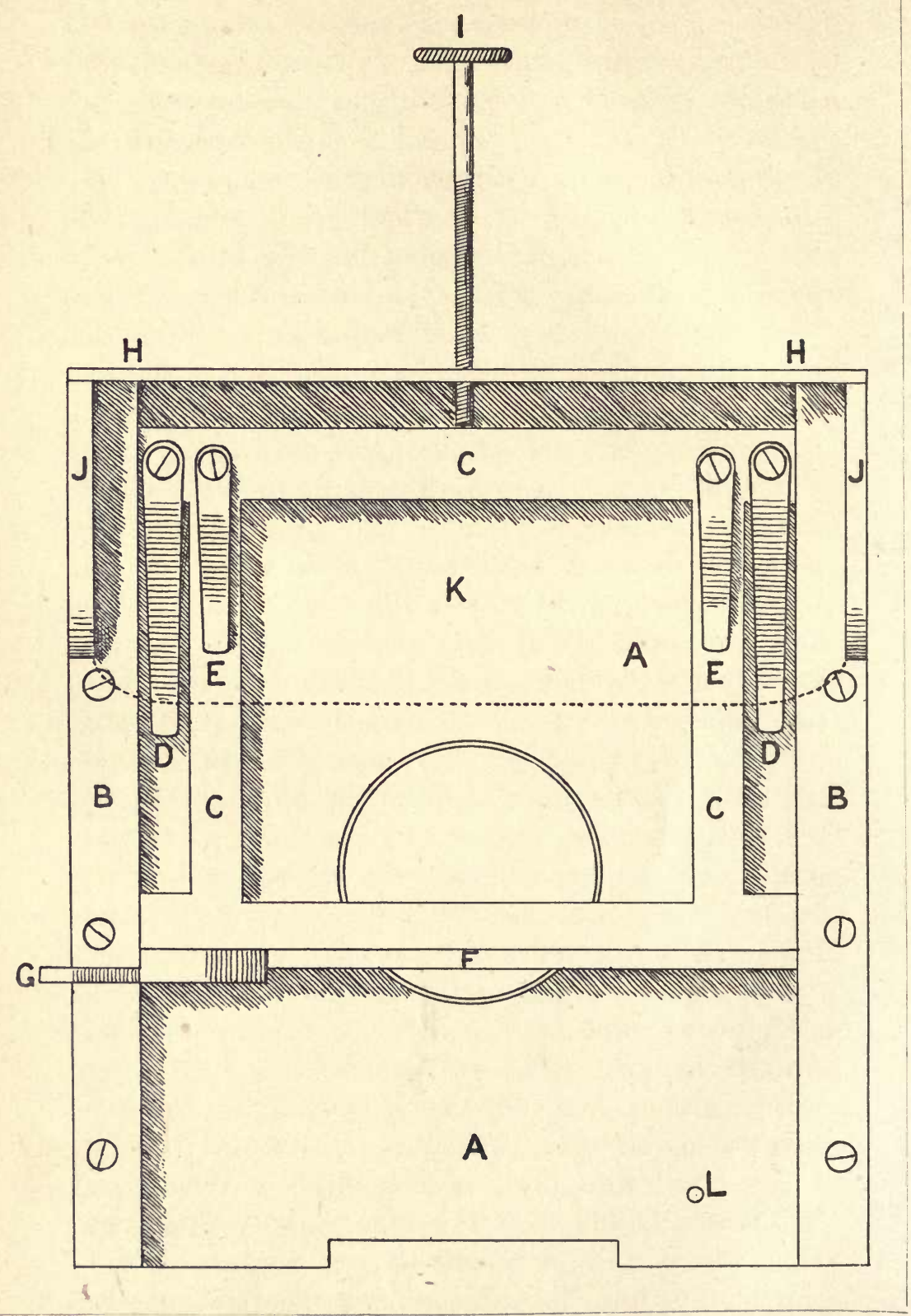

COLE'S DIATOM MOUNTING STAGE. 
devices for selecting and transferring diatoms, \&c., to the cover were both cumbersome and uncertain in their use and action, and that diatoms when picked up by means of a hair, a bristle, or a brush, of which the point was coated with gum, or some other sticky substance to which they adhered (or did not adhere), resulted in constant loss of rare and valuable forms, in course of transference to the cover, breakage, loss of time, and general uncleanliness, he invented, for his own use, the "Diatom Mounting Stage," of which the accompanying diagram is an exact and full-size illustration.

This stage is, of course, intended to replace the ordinary mechanical stage of the microscope, and that stage being temporarily removed, the diatom stage is firmly fixed to the flat table of the main stage by means of two screws passing therethrough and screwed into the diatom stage. The stage must, of course, be so made that the central circular orifice shall precisely coincide with that of the stage of the microscope with which it is to be used. Its description and the manner of its employment are as follows:-A A is a flat brass plate with a central circular orifice for the passage of reflected light from the mirror of the microscope. B B are grooved side-pieces, in which slides, very smoothly and evenly, the frame C. This frame is retained in position at any point in its upward or downward movement by two springs $\mathrm{D}$ D. It has also two smaller springs $\mathrm{E} E$, the use of which will be described presently. $\mathrm{F}$ is a slightly raised bar ending in a handle $\mathrm{G}$. $\mathrm{H}$ is the raised upper rim of the stage through which a screw passes, I. J J are the raised sides of the upper portion of the 
stage; and $\mathrm{K}$ is a piece of glass (its termination being represented by a dotted line) which slides into grooves in the sides, $\mathrm{J} \mathrm{J}$, and which is intended to secure, and practically does secure, absolute cleanliness to the slide during its preparation.

Now, the stage being fixed in its place by its two screws, an ordinary glass slip ( 3 in. by 1 in.) is taken, and by means of a turn-table a very minute circle is marked, with a writing diamond, precisely in its centre, upon this circle is placed the smallest possible drop of distilled water, and upon this drop of water the cover, on which the diatoms are to be mounted, is placed, and of course firmly held in its position by capillary attraction; care must be taken that the circle on the slip shall exactly coincide with the centre of the cover. The slip is then to be placed under the springs $\mathrm{E} \mathrm{E}$, and the stage must be so fitted to the microscope that when the rim $\mathrm{F}$ of the sliding portion of the stage is drawn down by the handle $\mathrm{G}$ it is stopped by a pin $\mathrm{L}$ when the small circle under the cover on the slip is precisely in the centre of the field of the microscope. The slip being firmly held in its position by the springs $\mathrm{E} \mathrm{E}$ the absolute centrality of the "mount" is ensured, if care be taken that the cover is not moved. Now, having spread and dried some diatoms upon another slip, place the slip upon the lower portion of the frame C C. This slip can be freely moved, and if the screw I is screwed out to its extreme length it is obvious that if the frame $\mathrm{C} \mathrm{C}$ is raised to the lower end of the screw the lower edge of the movable slip can be passed across the field of the microscope and any desired diatoms selected from it. After removing 
the diatoms from that line the screw is to be screwed down a little so as to prevent the frame sliding up so high, and this gives another line of diatoms for selection, and this can be repeated until the entire slip has been thoroughly explored. In this manner nothing can be overlooked, since the action of the screw ensures the return of the slip to precisely the same spot. Now, the cover on which the diatoms are to be mounted is to be previously prepared by placing upon it a small drop of the cement already referred to, which cement has to be kept moist during the selecting and arranging the diatoms. For this purpose an ordinary glass tube with a bulb is to be procured and mounted in a piece of brass tubing, having a ball and socket joint, and which is to be made to fit into that part of the microscope stand which is occupied by the side reflector when in use. To the shorter end of the glass tube a piece of indiarubber tubing is to be attached, and a small piece of glass tubing, to be used as a mouthpiece, is to be inserted into the other end of the elastic tubing.

Now this "breathing apparatus" being fixed in its place on the stand of the microscope, the longer end of the glass tube is to be placed about three inches from, and level with, the thin cover on the glass slip, and the frame C C drawn down to the pin L; now breathe (do not blow so as to blow away the diatoms) upon the cover, and it will be found that the moisture from the breath will at once liquefy the cement, and with a bristle mounted on the cedar stick of a camel-hair pencil-as already described-carefully remove all dirt, if any, from the cover, then from the lower slip select a diatom 
and if necessary, wash it on the slip in the moisture from the breath, which moisture will also cause the diatom to adhere to the bristle; now draw down the sliding frame and place the diatom on the cover. In this way any number of diatoms, \&c., may be transferred from the lower slip to the thin cover and arranged as desired, and when the moisture from the breath has evaporated, it will be found that the forms are firmly affixed to the cover, whilst the glass roof over the "mount" has preserved it from all danger or dust during its preparation. The cement with which the diatoms are to be affixed to the thin cover is made thus:- In 1 ounce of distilled water dissolve 2 grains of best gum arabic, without heat. When the gum is dissolved add 3 minims of glacial acetic acid, to prevent fungoid growth, and the least trace of sugar to prevent the solution from cracking as it dries. ${ }^{1}$ The solution should be carefully filtered through the finest filter paper, and the filtering repeated at intervals of a few weeks.

For years experiments were in progress in the hope of discovering a medium in which to mount diatoms, which should be more satisfactory, in respect of its refractive index, than Canada balsam, and equally reliable and permanent; many such media have been tried-phosphorus, balsam of tolu, oil of cloves, and others; but it is unnecessary to enter into details concerning them, since the

1 For affixing delicate insect dissections to the slip or cover, this cement should be made with 8 grains of gum arabic to the ounce of distilled water, and for polycystina or foraminifera with 10 grains, and for sponge spiculæ with 6 grains to the ounce. 
desired results have been obtained by Dr. Van Heurck, who succeeded in providing an admirable substitute for balsam in gum-styrax. It will probably be found that diatoms mounted in gum-styrax are less liable to accidents than balsam "mounts," as the latter becomes resinous in time, and the covers are liable to "spring," the result of which is the appearance of prismatic colours in the balsam, which are not only a great eyesore, but sadly deteriorate the slide. Gum-styrax, the method of preparing which for use has been described at page 105, may be considered absolutely permanent and unalterable. The solution is used in the same manner as Canada balsam. Styrax solution is even easier to work with than balsam, and air-bubbles are not produced in it by the application of heat. Monobromide of naphthaline as a medium in which to mount diatoms, and the method of its application, have been described at page 105 .

\section{Cleaning and Mounting Polycystina and Radiolaria.}

The siliceous shells of these lovely organisms, the beauty of which is only equalled by their variety, are found in profusion, and intermixed with diatomaceous valves, often of extreme beauty and rarity, in the deposits (or so-called " earths") of Barbados, the Bermudas and the Nicobar Islands, whilst an entirely new and magnificent deposit was discovered by the late Captain Perry, of Liverpool, at Jérémie, Haiti, which contains not only a large number of novel species of polycystina, but many entirely new varieties of diatoms. The celebrated "Challenger" 
dredgings also gave to science innumerable and splendid varieties.

The polycystinous "earth" should be broken into small pieces, about the size of a nut, and boiled for half an hour to an hour in a strong solution of common "washing soda," the disintegrated portions being poured off into a large vessel containing clean water, from time to time, and the boiling in soda repeated, as also the pouring off, until the whole mass is perfectly broken up. When the disintegrated matter in the large vessel shall have thoroughly settled down it should be subjected to several washings, in order to remove the soda, the material being allowed to "settle" thoroughly after each washing. It should then be removed to a beaker or wide-mouthed bottle which should be filled up with water, and after being thoroughly stirred or shaken up, the material should be allowed to settle for thirty seconds only, in a depth of 6 inches of water, and the supernatant fluid and its floating particles poured off into a large vessel; this process should be repeated three or four times, and will give the heaviest density of sand and polycystina. Repeat this process with the matter in the large vessel, allowing it to settle for two and a-lialf to three minutes, and the density containing the small polycystina will be obtained. Subject the remaining matter to like treatment, allowing it to settle in 6 inches of water for twenty minutes, and the density consisting of the débris of polycystina and of diatoms will result. Now boil each separate sediment in nitric acid for fifteen to twenty minutes, remove all trace of acid by repeated washings, and finally boil each density in a weak solution 
of bicarbonate of soda in a test-tube of large capacity for an hour; this will remove all flocculent matter, and after repeated washings, perfectly clean polycystina will be obtained. The heaviest density consisting of sand and the largest polycystina should be placed in a test-tube with about 3 inches of water and subjected to rotatory motion, this will cause the polycystinous shells to rise above, and to free themselves from, the sand, and they can be poured off into a wide-mouthed bottle or beaker, the sand being left at the bottom of the test-tube. This latter process should be carefully conducted and repeated several times in order that no large and perfect shells may be left amongst the sand. Each density should then be bottled in distilled water. Polycystina may be mounted in balsam - in the same manner as diatoms - or "dry." Beautiful slides can be produced by calcining the shells upon a piece of thick platinum foil, or in a small platinum capsule, and mounting them "opaque." The neatest and best method of preparing such slides is to make a disc half an inch in diameter in the centre of a slip, allowing it to harden for some days. A half-inch cover is then to be cleaned and a sufficient quantity of balsam, thinned with turpentine, put upon it; the polycystina are then to be placed in the balsam in sufficient quantity to form a surface over the cover when evenly spread upon it; the cover with the polycystina is then to be put aside (as recommended with diatoms) for twelve or twenty-four hours, in order that the balsam may thoroughly permeate the forms and all air escape; the balsam is then to be hardened by gentle heat, as already described, 
and the cover is to be laid upon a slip, with the balsam upwards, and held over the flame of a Bunsen burner until the balsam is liquefied; the polycystina are then to be evenly spread upon the cover by means of a needle; when the balsam is cold a small drop of benzol-balsam is to be placed upon its surface and a clean half-inch cover to be warmed and carefully lowered upon the balsam. The polycystina will thus be mounted between two covers; now turn the "mount" over, so that the under surface of the lower cover shall be upwards, and coat this with asphalt, put the mount aside for a day or two, in order to allow the asphalt to become thoroughly hard, then having made in the centre of a slip a disc of asphalt of the same diameter as the "mount," and allowed that also to dry thoroughly, put upon this disc a small drop of (cold) "French liquid glue," place upon this the asphalted under surface of the " mount," and when the glue is quite dry finish off the slide with either asphalt or white zinc cement.

1. This is to be obtained from any chemist, and is a most admirable, reliable, and cleanly cement. 


\section{CHAPTER VII.}

\section{On Microscopical Drawing and Painting.}

A Great teacher has said "Drawing should be considered not an accomplishment, but a necessity. Learning to draw is learning the grammar of a language. Anybody can learn the grammar, but whether you have anything to say is another matter." To the naturalist this accomplishment is of great importance; accurate illustration adds to the value of written description. At every point the microscopist is sensible of its deeper significance. Such a control quickens the perception, excites exact observation, and creates an interest beyond research, admiration, or curiosity. The compactness of the vision presented by the microscope so rivets the attention, that changes, disclosures, development of activities, in organisms often lost and swept away, after cursory examination, rouse in a zealous observer an impatient desire to possess some power, beyond words, to place on paper a memorandum or record, however rough, of things rarely discovered under the same eonditions. This ability is a result of practice. There is no 
royal road but that traversed by enthusiasm and earnestness. Sketches from the hands of a dexterous microscopist, marking first impressions, are often more valuable than, and superior to, the formal work of the mere draughtsman, who may not even know the significance of the subject, especially when the result is a replication of drawings made by the actual observer. He necessarily falls into one or other of two errors; he mends and improves, or obscures material points by drifting into formal monotony; a microscopical draughtsman must essentially be a microscopist, and work direct from actual observation, completely understanding the matter before him.

There are three well defined characteristics of microscopical representations, drawings of tissues, or minute organisms, requiring for elucidation high powers, delicate conditions of light, conducted under careful observation and technical skill, satisfying the highest biological research, in its progress demanding rigorous precision; then, rapid sketching, catching features, graphic memoranda ; without hesitation, or the assistance of the camera-lucida jotting down, and washing in, with tints, unexpected appearances, this readiness should be cultivated by those desirous of adding record to observation; many most important phases in the sequence of activities have been seen and passed over, when a few rough lines would have induced and helped further research, but beyond this tentative work, and the stern formality of scientific requirement, is a finished "picture;" at this crucial point the capability of the microscopist and artist blend, involving knowledge of the subject, 
the arrangement of optical apparatus, judgment, and study in the methods of procedure. . A drawing may be true in its scientific aspect, and possess artistic features of decided interest-the one may incorporate the other. The illustrations of Mr. Gosse's books are instances of this peculiar quality. "Still Life" has arrested the attention of artists of all time, from Missal Illuminators to Royal Academicians; such results have no scientific import, but like all art products, awaken gratification in appreciating the power applied in producing their essence, and without degrading the legitimate functions of the microscope, it is possible to extract from its revelations, models of exceptional charm and excellence, associated, moreover, with scientific value. Although the bias of an expert microscopist and practised artist may not often touch the same mind, it is certain that when a keen perception is directed to complications of beauty, with rare conditions of light and effulgence of colour, the instrument becomes the very touchstone of artistic feeling, and beyond mere beauty (which, in visible nature, is inexhaustible) there are revelations of structural form, quaint elegancies, mysterious changes of tissues, and embryological developments, under radiances, hidden, not only from ordinary familiarity, but even from the cognisance of many who have not had the opportunity of exhausting the resources of a fine instrument, with all its accessories. It may be urged that few have the ability to approach art of this description, but the power of drawing quickly develops itself, especially when stimulated by special and eager interest, concentrated on special objects ; no one led by inclina- 
tion to contemplate what may be seen under such circumstances, can be destitute of an appreciation of art in its most exalted sense. The education of the eye (the basis of æsthetic culture) as exercised by the fascination and mental excitement of microscopical research, progresses in a degree hardly yet understood or appreciated; every student has within his mastery this power; the manipulation of the instrument, the means of display, the use of materials, are matters of expertness, and are extensively self-taught; possibly some instruction may do more than groping alone, but in the end experience is the best master. It is proposed in this essay to detail such an actual experience in words, directed more to mental judgment than technical education.

A microscopical drawing may be absolutely true, and an artistic grace secured, by preserving line for line what is actually presented, assuming the preparation to be fairly perfect; in other words, not drifting into a stilted diagrammatic style, or wandering from close observation, because the subject appears to have a certain regularity; no two cells, vessels, or fibres are absolutely alike; to give "life" to a picture, every part of the structure should be a portrait, the pencil deviating from accuracy melts into falsity and confusion, uniformity is fatal, and obscures important differentiation of parts ; again, in order to delineate what is expected, or wished to be seen, aiming at "correction" is to be avoided; it is better to draw imperfections, if they be present, an overlapping or torn structure often reveals an important fact, so patent is this, that a "fabricated" drawing may be detected in a 
moment, especially of diatomaceous or infusorial forms-a broken fragment, a solitary individual is the clue to a perfect whole, or group; such built up arrangements have no charm beyond technicality. A good representation possesses a mingled quality of accuracy and imperfection, a paradox, which stamps its value! Suppose a preparation of vertical section of human scalp of rare excellence, double stained, disclosing beauty in many perfect hairs traceable in their course direct from the base of the bulb, embedded in the follicle, and emerging from the cuticle above. In cutting a section of such delicacy it would be impossible to avoid slicing through a hair or two diagonally, thus leaving the tops of some, the ends of others; this result or defect is a feature of significant interest from an art point, faithfully copied it gives life and character. In a diagram, the imperfection, by comparison with perfect hairs, might be remedied, the mutilated parts "restored;" but such an interfer. ence destroys at once the graphic quality of the picture, adding nothing to its scientific interest. Absolute accuracy in depicting what is presented may, however, in some cases, be qualified, and truth evolved by a knowledge of the structure as it should appear, particularly in cellular tissues, in close contact. In such cases the artist ought to be cognisant of elementary forms, as arranged under contiguous pressures, and the position of spherical, oblong, or cubical elastic cells, as affected by juxtaposition in, over, or under spreading layers. Coupled with the perspective of such conditions, this facilitates progress. In opaque subjects, under binocular vision, where the rotundity of a reticulated surface fades 
in dimensions, and shadow, in different lines, this abstract knowledge is important, and should be acquired, as many objects could not be effectively represented without its study-always keeping to general appearances; it is an ability which removes difficulty in unravelling the disposition of parts, especially under high powers; when sections are cut either too thin, slightly oblique, or disrupted by the knife, the mechanical interferences of parts, when understood, may be restored. The functions of an artist, cognisant of a condition of antecedents may be fairly exercised in the progress of a drawing, but it must never trench upon absolute truth and discrimination in treatment-a drawing may be ruined in a moment by a false line involving impossibility of structure; to a critical eye, this is fatal. In fine work, dealing with malpositions, shrinkage of tissues, disseverances and pseudoappearances-inevitable even in the finest preparations - the utmost judgment is required.

The effect of a microscopical drawing is enhanced by its inclusion in a circle-surrounded by a black margin-forming a square. The size of the circle is important-it may be too large, or too small; experiment proves that a space three inches and three-quarters in diameter approximates nearest to the impression made on the mind of a "field" as seen with a $B$ eye-piece; this circle may encompass magnifications under any power. A metal plate four inches and three-quarters square, with an opening of the dimensions given, should be procured, this ascertained gauge will soon prove a necessity; placed on a drawing block, a pencil swept round the circle and outside the square gives 
the interior for the drawing and the lines for backing with indian ink-these discs should be prepared before the work is commenced, and the importance of this arrangement will be shown hereafter.

The help derived from the camera-lucida is strictly limited, it cannot be employed beyond a certain point, no elaboration can be effected by its prolonged use, it should be discarded the moment its legitimate purpose of marking points and positions is achieved; those experienced in its employment always feel a sensible physical relief, and "breathe again" when it is set aside to settle down to the earnest work of direct vision. The application of the camera-lucida to the instrument is sufficiently familiar; the microscope, if a binocular instrument, having been rendered monocular by withdrawing the prism, the camera-lucida is slipped over the A eye-piece-which should always be used (higher eye-pieces expand the field beyond the fair range of the instrument). The object being clamped, the microscope is depressed into a true horizontal position (if not, a distorted picture would result), and the lights adjusted; the distance from the object to the eye-piece should be nearly equivalent (if anything a little more) to the reach from the eye-piece to the paper. With a microscope standing ten or twelve inches high (a Ross No. 1) this condition would reveal the phantom of the object, outside or about filling a circle of the dimensions given on the drawing block; if any difference of over, or within, lapping appear, it may be remedied by raising or otherwise adjusting either the paper or the microscope so as to obtain a perfect coincidence of the vision and the circle; the importance of a measured 
disc is now manifest, proportion is affected by distance from the eye-piece, and with this gange and a stage micrometer, a drawing may be kept within measurable bounds; difficulties have existed as to amplifications expanded by the camera-lucida; absolute accuracy may be ascertained by the use of micrometers as far as the eye-piece is concerned, but the known diameter of a circle on which the image is projected is an easy factor in such calculations, beyond this, the circle is mechanically useful, as, if the block should slip when using the camera-lucida, there is an ascertained line for readjustment. The light on the paper should be in excess of that from the object; speed and precision are essential, quickly make recognised points and lines, never attempt to draw detail, nothing fatigues the eye or distracts the mind more than the prolonged employment of the camera-lucida. No adadvance can be made by its continued use, any attempt at elaborate work ends in confusion. Cultivate the "knack" of seeing at the same time, and in the same position, the reflections and the image of the tracing point (the hardest pencil, sharply cut); it is not necessary to strain the sight to keep the entire field always in view, there is a condition of steady gazing, the eye not too close to the prism, when PARTS of the object can be taken separately, but this is a result of the facile use of the instrument. The neutral tint glass or any form of reflector, giving only one, and consequently, a reversed image, is useless for afterwards continuing a drawing from direct vision, but with any description of camera-lucida, the pencil, once placed on the paper, should not, if possible, be lifted until all determining 
lines are fixed; the eye (unsteady at the best) and the pencil point must be in unison. Keeping the pencil on the paper preserves "the place."

In arranging any object for drawing, it should be sufficiently magnified to show everything bearing upon its elucidation, and, as a rule, an isolated subject, a complete form, ought to occupy, as nearly as possible, the entire field. Some specimens necessarily overflow the circle-surfaces of injected preparations, botanical sections exhibiting features requiring the highest magnification consistent with the preservation of a focal plane which, obviously, cannot be fairly disclosed (except at a loss of important detail), are beyond the scope of the circle of popular survey. It must then be abandoned, the drawing spread out, and made in sections by shifting and combining visions. Using the cameralucida any part may be carefully drawn, making two or three (if angular, the better) prominent points, corresponding with similar appearances in the subject. These marks or tri-angulations (as near the margin of the field as possible), must be remembered, the position of the object is then moved by stage adjustments, and another part of the field arranged, the included marked points are coincided, by shifting the block of paper, and further outlines expanded; in this way the camera-lucida may be used under high powers with four or even six combinations of vision-and the parts, with care, will "read into" each other, and result in a drawing of considerable dimensions, perfectly mapped, and true in contour; it may then be continued part by part.

After faint outlines and points of certainty are 
securely indicated, the microscope is placed in position, and with $\mathrm{B}$ eye-pieces drawing from direct observation commenced; prolonged work is facilitated by removing the caps of the eye-pieces; when attention is continually diverted from the instrument to the pencil, the fatigue is lessened by keeping the eyes some distance from the glasses, cultivating a faculty of losing the recognition of the entire objects, only directing the alertness of vision to the particular part under consideration; in fact it is not necessary, nor is it prudent, to strain the sight to keep the full blazon of the field under observation, and this rule may, with advantage, be applied to the general use of the microscope. At this point, steady work commences, faint camera indications are studied, lines corrected and strengthened, either with pencil, or better, a fine sable brush or pen, carrying a mere tint of indian ink or "Payne's Grey." Extreme care is necessary; no mistake of line can be permitted; paper, intended for such drawing, and delicate after colouring, does not permit erasure, or the contact of any rubbing out substance; and consistent with the subject, too much fine line cannot be put into the work; no attempt at shading, either with pen or pencil, must be attempted. The lines being perfected, and the subject, as it were, "modelled," the painting may now be cautiously commenced. The absorbent quality of paper (well known to those accustomed to water-colour sketching), interferes with, and sometimes assists in securing artistic results; without entering into the rationale, but bearing upon the point, it may be mentioned that no wash or even line should be superimposed upon or carried 
over another until the first be perfectly dry; stippling should show a granulated appearance, lost when touches are allowed to run into and become absorbed by each other.

Illumination, and its diversities for art work, are of as much importance as the amplification. Power and light should be adapted to each other, and to the character of the subject, its mode of preparation, and what it is expected to reveal. The light, whether from gas (argand burner), or oil, should be capable (in the case of gas, by means of flexible tubing,) of being placed in every possible position, from the surface of the table to, or even above, the level of the stage. Ordinary transparent objects, under low powers, are sufficiently shown with transmitted light from the mirror, modified through a diaphragm of waxed tissue paper; ordinary preparations of insects cannot be better displayed; the best reflected light is from the side speculum, collected from a flame through an intervening planoconvex lens, on a separate stand. In all observations, even the simplest, accuracy of light-focus (often neglected) is important. For powers beyond the half-inch, transmitted light is aided and inproved by the purity and control afforded by the achromatic condenser, an instrument in the hands of novices, not always well managed, or sufficiently appreciated; focussing on the same plane as the object, the source of light, it is capable of regulating intensity, purity, and deviation of rays-by apertures and stops, with which it is supplied-and thus the most varied combinations may be secured. Its use should be thoroughly mastered, as it produces the most beautiful, instructive and even amusing 
effects. For instance, with a half-inch objective and full aperture, carefully focus, on a ground glass slip, the flame of the lamp, now interpose a dark stop which should occupy, in the centre, about onethird of the field; removing the slip, replace it with a group of (say) volvox-globator; the plants will be seen rolling from the outer ring of pure transmitted light into the central black disc, where they appear like emeralds; free-swimming rotifers wili pass backward and forward, from the outer ring of parallel rays, into the eclipse of the dark stop, where they become by oblique radiations, selfluminous; no finer example-as showing in one field, at the same moment, two extremes of illumination-could be placed before the microscopical artist, or an ordinary observer, to prove what may be effected by an adept in the use of this beautiful instrument. With low powers striking presentations of artistic illumination are under easy control; in particular, the use of the paraboloid combined with light from above. An experienced microscopist is familiar with all these methods - but the artist, alert and eager for experimental conditions, often hits upon effects not generally applied, possibly sacrificing scientific truth to æsthetic desire; a result of positions, and foci of illuminators - their accurate or eccentric adjustment, cutting off central or peripheral rays, dispersing or half obscuring light by intervening transparencies. The importance of such combinations is paramount in the examination of semi-opaque objects immersed in a thick bed of medium, without pressure. These preparations are in parts dense, even solid, combined with tissues of extreme delicacy and transparency; nothing being 
crushed, they shew the impossibility of revealing the correlation, or association of parts, by mere transmitted light-or in any way, except (may it be said) by artistic discernment. The head, and surrounding parts of an insect, prepared in this way, with pure light from beneath and above, discloses a combination of form and colour of surpassing beauty; the blaze from the speculum sweeps over the opaque parts with reflections revealing the most exquisite tints, while the paraboloid shows, in actual perspective, the parts beneath in all their natural colour, and bathed in refulgence. An opaque polypodom, touched by such reflections, while the extended polyps are illuminated from below, is another instance, amongst many, of beauty, exalted by light.

For purely opaque objects, the only good light is from the speculum, by no other means can the finest effects of colour and shadow be obtained. It should be fitted to the stand of the instrument, not to the stage, nor should it slip on the front of the objective. On the stand it can be moved without disturbing the object or the focus. The old fashioned Lieberkühn cannot be used; it requires an object to be prepared in a particular way, and as an illuminator is palpably defective; the light completely surrounds and enwraps the object; brilliancy is present, but no contrasts. In using reflectors, the lamp should be placed close to the level of the stage, within easy reach of condenser and speculum.

In painting purely opaque objects under top light the treatment of background deserves attention; eggs of insects or parasites are generally attached to fragments of wood, leaves, cuticles, hair or feathers, it 
enhances the effect and beauty of a representation, if such details are carefully painted, and the rest of the field delicately stippled up with indian ink, to the edge of the circle. This applies to many subjects-threads of algæ, or vegetable stems supporting such objects as fixed rotifera, polyzoa, \&c., introduced into a drawing, add greatly to the interest and make most attractive pictures. Any prepared mount or specimen should be as perfect as possible, and considerable experience is necessary in order to decide what is fairly good as a preparation and worth drawing. Common objects of easy procurement from the woods, the garden, and the stream, are exquisite models for the draughtsman, their excellence, interest, and freshness are necessarily superior to even the admirable results now obtained by professional preparers, aided by mechanical appliances, and rare skill in the use of reagents and staining fluids.

It is obvious that objects under polarised light, are practically beyond the power of faithful delineation; in all painting, whether in local tint or shadow, purity of colour and the preservation of brilliancy is of the first importance; in order to render, beyond a mere semblance, any subject under the polariscope, it would be necessary, if such a power were possible, to dip the pencil into light itself, and an insuperable difficulty exists in the permanent preservation of the adjustments necessary for future work, the slightest touch, or alteration of any part of the instruments, and even an obscure change, beyond all control, in the quality of the source of light alters the entire gamut and consonance of colour, impossible to re-establish, yet, 
if selenite films be dispensed with, some results may, with care, be recorded; petrological preparations, the dichroism of crystals, sections of shell, bone, scales, horn, and other semi-transparent organic structures of varying densities, reveal points of interest only seen under such conditions and may be noted; but considering that the most exalted light at the command of the artist, is the white of the paper (in all cases, to be jealously preserved), and that the polariscope discloses the purest coloured lights, associated with complementary tones of every gradation, it is clear how futile are the resources of the palette to depict the lustres and unisons of tones as revealed by this fascinating instrument.

Structure and its thoughtful exposition is the limit of draughtsmanship, and it is here that the photographic lens as a delineator fails; the superiority of work produced by a hand guided by cultivated observation as compared with a photograph is the operation of a mind capable of expressing combined and superimposed tissues, in having at command a control and adjustment of various planes of surfaces, and without militating against scientific truth, seeking for, and obtaining even picturesque effects, this important power is felt when searching the depths of an opaque injection, or peering into intricacies of tissues. The objective used in microphotography, especially if it be a high power (unlike the penetrating quality of an ordinary portrait lens), is strictly limited to one, and that a very delicate focal plane requiring a fine and FINAL adjustment, enhanced by the difficulty that the visual and chemical foci of microscopic objectives do not coincide, entailing a manipulation which 
never touches perfect precision; on the other hand, a draughtsman may arrange a minute and just perspective of parts, absent in a photograph, anticipating the presence of relative parts, and having at command the fine adjustment, he can feel his way, conscious that at the slightest touch a fresh point, perhaps an important revelation, flashes into sight, supplying a link to the better understanding of the whole. A drawing, produced under thoughtful guidance, conveys to an appreciative observer an attraction totally absent from a photograph; the latter may possess the important and essential element of proportion and freedom from exaggeration, but exactitude is never absent from a drawing disclosing understanding, and conscientious treatment.

There may seem little or no analogy between landscape and microscopical painting, but the same principles are involved-points of sight, effective light, general entourage-possibly a " preparation," dealing with unusual and unexpected complications of line, embracing physiological difficulties, requiring delicate conditions of luminosity, demands a deeper judgment, for it is often necessary to prepare the mind by careful and prolonged study, before the paper is touched; especially in considering and anticipating difficulties of representation, and how they may be overcome-delicate structures, under the most careful illumination, often appear as streaks of LIGHT, when a slight touch of the condenser may reveal distinct lines. These are points to be studied; in fact, the subject should be " gone over" and arranged in all particulars, so that it may not outstrip the power of the pencil. All materials 
should be of the finest quality, the paper, hard, thin, smooth, and unglazed; delicate pencil drawings may be made on Bristol board, but such or any glazed or hot-pressed surfaces are totally unfitted to take colour. Fine drawing paper is preferable, when blocks are used each surface must be examined for imperfections with a hand lens; a delicate painting may be ruined, at a critical point, by an imbedded hair, an abrasion, or minute speck; in the manufacture of these blocks, it has been found that in cutting up and folding the paper the true surface is not in every piece placed uppermost. For important work it is safer to select sheets strained in the usual way, in a small-sized folding drawing frame. Paper improves by age. If of undoubted antiquity, it fetches high prices. It is impossible to render satisfactorily, on a white surface opaque preparations showing minute injected anastomosing veins, arteries, or glands, the dark interstices separating them, cannot be drawn, or picked out, without sacrificing the regularity or destroying the uniform diameter of the vesselsbut such subjects may be effectively painted on a dull black paper, which may be previously pasted on a drawing block under pressure, using opaque or body colour, vermillion, yellow ochre, Antwerp blue, and carmine, combined with and regulated for substance and tint, with zinc white and gum water. Payne's grey, with zinc white, produces the peculiar shadowy hyaline tone so often seen as a substratum in such preparations where semitransparent spongy tissues are involved-fine effects of receding distances -in following the depths of structures, may be produced by its use. Numerous 
brushes of sable are required, the hairs short and coming to a fine point, they should be of the best make, no brush that has touched indian ink can be used for delicate colour, and those employed for carmine, yellow, and blue, should be marked and kept distinct; the same applies to pens, often required, but the pen carrying colours must be used with extreme discretion. If a fine line can be obtained with the sable, it is of higher quality, moreover with the handy pen the temptation is great to obtain hurried results by strokes and dots, but for pure black and white memoranda, or representations requiring speed, nothing can equal a fine pen charged with indian ink or neutral tint, remembering never to approximate or cross a line until it be properly dry; with this precaution a pen drawing may approach the semblance of an etching. The colours should be dry cakes, the palette preserved as pure as possible ; moist pigments rubbed from pans become contaminated, and even dry cakes should be kept separated; loose and in contact they chip and soil each other. Quality is all important, use only those which are " transparent." Manuals on painting contain lists of recommended pigments and their qualities, and generally receive no attention; for the work in question it may remove difficulties to remember that important colours are neutral tint, Payne's grey, Antwerp blue, carmine, scarlet lake, yellow ochre, Hooker's greens, Nos. 1 and 2, and raw Sienna-colours to avoid, vermillion, cadmium, the umbers, emerald green, and Vandyke brown. These are densely opaque, and " load" too heavily for delicate work; a good test is to rub a portion of each cake of a 
well-furnished box on a clean porcelain palette, side by side. When dry, those which appear dull and dusty (however useful they may be in landscape in large thin washes) reject. Everything may be accomplished with the remainder. There is no difficulty in conducting a painting by artificial light when conversant with the character and combinations of the few colours really required, a precaution, however, is necessary in painting tissues stained artificially with logwood or aniline dyes. These colours are very deceptive, and differ in appearance under degrees and qualities of light. Logwood stain (often used) in daylight has a blue tinge, under the lamp it appears as a decided port wine tint, and a difficulty may (in fact, does) ensue in matching day and lamplight work. When the entire subject has to be painted in the same tone, cakes of "mauve" and "aniline blue" now to be procured may be used alone, and thus stained tissues can be painted under any conditions of light without falling into error. It need hardly be said that such abnormal colours are to be used exclusively for those special preparations, and should never enter into the composition of, or even touch a general palette required for natural representations. Indian ink must be of superlative quality, the difference in price, although not deadly, is great. A piece should be secured, regardless of cost, and treasured.

With practice in cultivating accuracy of touch, certainty of line, and ignoring the existence of "rubber" and knife-edge, no difficulty need be anticipated in drawing on wood, zinc, or lithographic stone. 


\section{CHAPTER VIII.}

\section{On Photo=Micrography.}

IT is not intended to claim any originality for the subject of this chapter, since the application of photography to the delineation of microscopical preparations is almost as old as the photographic art itself, extending back even to the days of the daguerrotype. Microscopists of the present generation should think of this, and while paying tribute to the patient perseverance with which their forerunners must have worked under all sorts of disadvantages, should blush that, notwithstanding all the recent advances and all the simplicity of the gelatino-bromide process, so few avail themselves of the facilities it affords for the truthful and beautiful delineation of the objects of their study. The chief reason for this neglect is probably the idea among the uninitiated that photography is a very complicated and difficult art, dependent upon a very uncertain condition in our climate - bright daylight-and that unless one had the necessary day-time leisure and were expert in ordinary photography it would be useless to attempt this special application of the art. It is to expose the 
fallacy of all this, and to show that any microscopist armed with a small text book and with a simple apparatus which it is quite within the bounds of possibility for him to make for himself, and having no leisure time but the dark winter evenings, can, after a few weeks' practice, produce pictures of specimens in his collection which, for absolute fidelity and beauty, are incomparably superior to the highest flights of the draughtsman's skill. In taking up the study of photography a beginning must be made somewhere, and the tyro's first efforts may as usefully, and with as great a prospect of ultimate success, be directed to this branch as to any other. It is a matter, not of doubt, but of certainty, that his first attempts will be failures, from under exposure, over exposure, forgetting to draw the slide-and therefore no exposure-fog, frills, stains, pinholes, under - development, and otber causes; but had he commenced with portraiture or landscapes, he would have had the same dismal record of good plates gone wrong, and would have had the additional gratification of many an unproductive tramp. Therefore we would say be not deterred from taking up this work because you are not a photographer. Commence operations and become one. It will, of course, be impossible here to give any elementary instruction in photography pure and simple. All that can be done is to describe such apparatus and processes as are special to this particular work, for all that is general a good text book of photography must be consulted. ${ }^{1}$

${ }^{1}$ Captain Abney's "Instruction in Photography," Piper and Carter, is one of the best, but there are several well known and more modern text books from which choice can be made. 
The apparatus employed is simple. It consists of a microscope of any ordinary construction, a powerful source of light, and a camera. The complete apparatus perfected and manufactured by Messrs. Swift \& Son, Tottenham Court Road, from whom price, description, and all particulars may be obtained, is here illustrated. The mirror of the

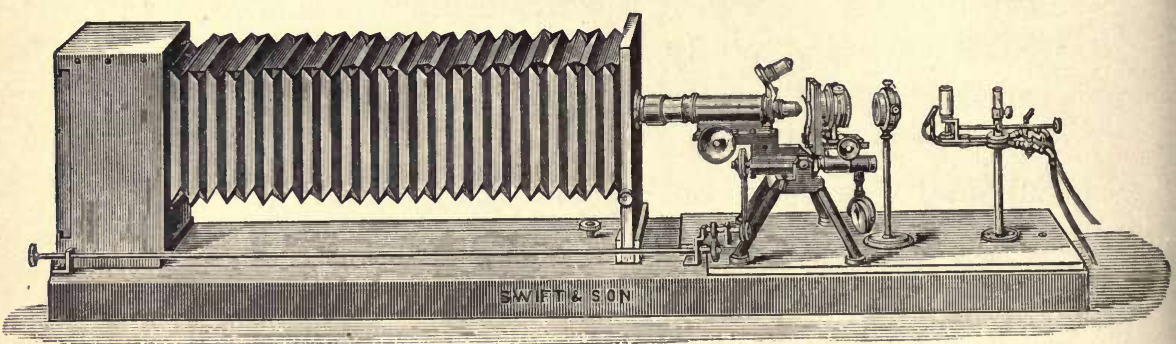

microscope is discarded except for special purposes, because the loss by reflection is very serious. The microscope is placed horizontally in a line with the source of light, and with its tube inserted by a light-tight joint into the front of the camera, which is supported at such a height that its centre coincides with the optic axis of the microscope. The object is held on the vertical stage by means of spring clips, and the light from the lamp is condensed on it by one or more condensing lenses. There are two chief methods in use. In one the eyepiece of the microscope is removed, and the inverted image is received on the sensitive surface where it is first formed. In the other the eyepiece is retained in its place, and the image first formed in its interior is formed again with additional magnification by the aid of the eye-lens. By the 
use of the eyepiece the advantage is gained that any ordinary camera may be used, and in consequence of the shortness of the distance between the focussing screen and the microscope, the various adjustments of the latter are accessible, while focussing, without any special arrangements. In order to secure the same amount of magnification or to cover the same sized plate, when the eyepiece is not used, the camera must be of special construction so as to be capable of extension to two or three times the former distance, and when so extended to-say a yard-the focussing screen can only be reached by rods and bands or intermediate gearing. For facility, therefore, the eyepiece method is commendable, but the impairment of the image and the loss of light due to the interposition of two additional uncorrected lenses are so considerable that we would advise the removal of the eyepiece for all but special purposes, such as the covering of a very large plate.

The microscope may be of any ordinary construction that will allow the body to be placed horizontally, and it should have a stop to prevent it going beyond that position. It should be provided with a coarse adjustment, by rack and pinion, and a very sensitive fine adjustment for slow focussing. Very much of the success of the work depends upon the excellence of the slow motion. It is always a very important part of the microscope, but for photo-micrography it becomes especially so. It should be free from the slightest lateral motion, lest, in focussing, the image be removed from the centre of the plate, a very small displacement of the object glass being sufficient to effect this, when 
working with high powers and a long camera. It must work with perfect smoothness and without loss of time in either direction. If the tube of the microscope can be shortened by unscrewing the part above the fine adjustment so much the better, for when working without the eye-piece, a long tube, especially if it be a narrow one, greatly contracts the field. This is one objection to the Jackson-Lister form of stand for photographic work, and the difficulty here can only be got over by selecting an instrument with a wide tube. There is another objection to the retention of a long tube, whether the eye-piece be employed or not. It is certain to give rise to a "flare" of light by reflection from its inner surface, and flare, whether arising from this source or from the setting of the object glass, or from the interior of the camera itself, is absolutely fatal to the production of clean pictures, and results in the diffusion of a uniform light all over the plate, which impairs the purity of the shadows and produces a general fog. One chief seat of this internal inflexion is the fine adjustment tube. No amount of dead blackening, or even lining with black velvet will completely stop it. The only thing to be done is to interpose along the course of the tubes of the microscope, and in the camera, and even in the object glass itself, if necessary, a series of diaphragms. These may be cut out of cards with gun punches, and glued to narrow rings of cork to give them a grip of the tube. They and the corks also must be painted dead black with water colour (lamp black), and their number, position and aperture so adjusted that a line drawn from the centre of the object glass to the edge of 
the tube when at its shortest, shall just touch the edges of all these apertures. Thus arranged they will not contract the field, and will not allow a ray of light to fall on anything but the front faces of the diaphragms themselves, whence it cannot be reflected to the plate. When the eye-piece is used the diaphragms must be differently adjusted, for the tube then practically ends at the front surface of the field glass, and its diameter is practically the clear aperture of that lens. A slight sliding backward or forward of the diaphragms will be sufficient to effect this adjustment. In the camera itself the diaphragms, if required, will take the form of sheets of blackened card with central apertures. 'The writer, who works with quarter plates ( $4 \frac{1}{4}$ by $3 \frac{1}{4}$ inches), prefers to make the aperture nearest the microscope small and circular, the next larger and shortly oblong, with very rounded corners, the next more oblong and with less rounded corners, and the last oblong with acute corners and the shape of the plate, but a trifle smaller. On looking through the whole length of over a yard of camera and tubes when so arranged, and with a blaze of light streaming in from the condensers, not a single stray beam can be seen, and nothing is visible but the object glass "full of light." Then when a plate is properly exposed and developed, the deepest shadows come out as clear as the glass itself, and the negative prints brightly and quickly.

When working with 3 , 2 , or even 1 inch objectives, the fine adjustment may be dispensed with, and then, if the microscope be of the old Ross pattern, the tube may be entirely discarded, and the objective screwed (by means of a short adapter, if need 
be, or simply wedged) into the arm that usually carries the tube. By this means the field is only limited by the aperture of the objective, and there is no possibility of flare from the tubes.

A mechanical stage, with concentric rotation, will greatly facilitate the adjustment of the picture in the centre of the plate. With objectives of higher power than $\frac{1}{8}$ inch, these mechanical motions become indispensable. Spring clips are required to keep the object in a vertical position.

The camera may be of a size to take plates from $\frac{1}{4}$ to whole plate size according to the worker's choice, but at first, at all events, if not permanently, the smaller sizes are best. The quarter plate size is most generally used, and plates of that size are always obtainable and are cheap, but we would recommend 5 by 5 inches as a more useful size, conforming more nearly to the circular form of the field, and to many objects, diatoms, echinus spines, sections of stems, \&c., which involve a great waste of surface when taken on oblong plates. By means of a simple adapter a camera of this size will take $\frac{1}{4}$ plates when desired, and these can be placed with their longer edges either horizontal or vertical.

The camera should be made with a bellows body capable of closing up to 6 or 7 inches and extending to about a yard, it will then be available for use, either with or without the eye-piece. To the front should be fitted the base of a smaller bellows of conical form, whose small end terminates in a wood, metal or cardboard ring, lined with black velvet and fitting smoothly and light-tight on to the outside of the tube of the microscope. 'The dark slide which carries the plate should be single, and 
constructed to take wet plates as well as dry. Unless a very large camera or a very small microscope is used, the camera will probably want blocking up to bring its centre exactly to the same height as the optic axis of the microscope. For this purpose a light support of pine should be constructed. It should be as long as the camera when fully extended, and should be graduated in inches along one edge. If polished it will keep cleaner and look much better than if left plain, and will not wear the edges of the bellows. On the top of this support the two ends of the camera may be made to slide, and may be secured in any position by set screws running in a central groove.

The microscope, camera, illuminating apparatus, \&c., are to be clamped down in marked positions on a base board, about 6 feet long, 11 inches wide, and 1 inch thick. The point of the base board, immediately below the position of the object when in use, should be marked, and the board should be graduated in inches from this point in both directions. The camera support should have a play of five or six inches to, or from the microscope, and when adjusted, should be clamped by a single turn of each of two screws, which hold it to the base board. To meet the wants of those who are content to use the eye-piece, Mr. Stanley, of London Bridge, has constructed a cheap apparatus, consisting of an ordinary $\frac{1}{4}$ plate bellows-camera, and a base board, to carry microscope and lamp, and with adjustable platform to bring the camera up to the height of any ordinary microscope. ${ }^{1}$

${ }^{1}$ Mr. Stanley also publishes a pamphlet, containing instructions for working dry plates, silver printing, \&c., which is worthy the attention of beginners. 
As to illumination, there is a great choice, but we may at once dismiss for ordinary work: Sunlight, as being too precarious, and necessitating mid-day leisure; Magnesium, as too expensive, and very difficult to focus by, on account of its trick of going out when left to itself. Electric arc necessitates the charging and discharging of 30 or 40 Grove's cells, a pleasure that can be appreciated only by those who have tried it. In favour of Incandescent lamps we cannot say a word. A 20-candle Swan lamp requires as powerful a battery as a small arc, and has none of its advantages. Its light is not concentrated, it is feeble and yellow. Lime light is the cheapest, least troublesome, and, on the whole, the best of powerful artificial illuminants. Our choice is therefore limited to lime-light, gas and paraffin. Of these, the latter is everywhere obtainable, gives a white light, and is all that could be desired for ordinary work with objectives up to $\frac{1}{8}$ inch. Our own arrangement consists of a paraffin lamp with $1 \frac{1}{2}$ inch wick placed close to one end of an oblong zinc reservoir. It is supported on a block about 20 inches from the stage. The glass chimney is narrow to allow of the close approximation of a plano-convex condensing lens of 3 inches aperture and 3 inches focus, which collects the light and transmits it in a slightly convergent beam. At a distance of 5 inches from the stage is placed a second plano-convex lens of 3 inches aperture and 5 inches focus. This further converges the beam on the object, and gives a brightly and uniformly illuminated disc of about $\frac{3}{4}$ of an inch in diameter, so that large objects can be well photographed under low powers. The object of using a short focus lens near the lamp is, 
of course, to intercept as large a quantity of light as possible, while the longer focus lens nearer the stage secures a larger disc than would be possible with a lens of shorter focus. The convex sides of both the lenses are turned towards each other. When working with the $\frac{1}{2}$ inch and higher powers, a further concentration of the light is effected by an achromatic condenser. If the edge of the flame is turned towards the condensers, the light is very bright, but there is a difficulty in illuminating a large field uniformly. If, however, the flame make an angle of about 5 degrees with the optic axis, this difficulty disappears without sensibly reducing the brightness of the field.

The arrangement in which an optical lantern replaces the simple lamp may be used with the advantageous results of shortening exposures, and facilitating focussing under high powers.

When working at night, there is no necessity to use a focussing cloth. The image is first received on a screen of the finest possible ground-glass, made more transparent by waxing the ground surface, but even this is too coarse for delicate focussing, and to get the final focus the screen is removed and replaced by a lens of about 1 inch focus held in the hand, but attached to a strip of wood, whose arms are brought up against the end of the camera when in use, so that, in shifting it about to examine different parts of the image, it is constrained to remain in one plane, and its distance has been so adjusted once and for all, by previously focussing the ground-glass when in position, that its focal plane is exactly that occupied by the sensitive plate. Mr. Stanley's arrangement for focussing consists of 
an ordinary ground-glass screen, with cover glasses cemented on to the ground side here and there, so that at these places the screen becomes almost as clear as plain glass, and parts of the image can be examined through them with an ordinary focussing lens. When the apparatus is used without the eye-piece, focussing is effected by turning a rod running underneath the camera, and actuating a roller round which, and also round a groove in the fine adjustment screw above, an elastic band passes.

The possible non-coincidence of the visual and actinic foci of the object glasses is a point that must be borne in mind, and each objective must be tested to ascertain to what extent, if at all, this non-coincidence occurs. This source of error is, however, usually eliminated by interposing, in the path of the beam between the two condensers when focussing, a glass cell with parallel sides, containing a solution of cuperic ammonic-sulphate. This will cut off all the rays of low refrangibility, and in nine object glasses out of ten, will be found to supersede any other correction, at all events, when paraffin is the illuminant. With the electric arc or other light in which the ultraviolet rays largely predominate, the case is different. The mode of testing for this want of coincidence, and ascertaining its amount, is to interpose the "copper" cell and obtain the best possible visual focus of some object with hard and sharp lines, with the glass to be tested. The

1 Prepared by adding ammonia to an aqueous solution of sulphate of copper, until the precipitate first formed is redissolved. 
cell is then removed, a photograph is taken, and if it shows any want of sharpness, the cell is again interposed, and by means of the fine adjustment screw, the object glass is approached a little nearer to the object, until the image as seen with the eye is about as indistinct as that on the photograph. Another plate is then exposed and developed, and will probably be quite sharp. If not, another slight alteration of focus must be made, and the process repeated if necessary, until at last the requisite sharpness is obtained. It should be then noticed how much the fine adjustment screw has to be turned, to bring back the proper visual focus, when the copper cell is used, and this amount should be recorded and applied as a constant correction whenever the lens in question is used.

So much for apparatus. Now we must say a few words about the photographic processes employed. Gelatine dry plates are the best suited to this work on account of their rapidity, the little apparatus required, the cleanliness of the process of development, and their being always ready for use. There are many excellent dry plates of various degrees of rapidity, and still more various prices in the market, and it is very much better to buy them than to make them for oneself. A rapid plate is most suitable, but there is no occasion for excessive rapidity. Gelatine plates must be exposed to none but deep red light, and the less of this they are exposed to the better. When unpacked they should be stored in grooved light-tight boxes. The smallest ray of white light finding access to the dark room will be fatal to success. If the operator works by night only, he will not need a special dark room. Any ordinary room with a closely fitting door, to 
keep out lamp light or gas light from the adjoining apartment (if any), will answer, provided there is not a lamp within a few yards of the window, and the moon is not shining brightly. The most convenient light to work by is that afforded by a paraffin launp, protected by a chimney of ruby glass, or a screen of good orange demy free from pin holes. This screen must be so arranged as not to allow a single ray of white light to enter the room in any direction, while it allows a proper supply of air to the lamp. If the operator wishes to work by daylight he must either darken a room or large cupboard entirely, and use the paraffin lamp as before, or he may glaze the window with ruby glass, and make it additionally secure by pasting a layer of yellow demy over it, or two thicknesses of bookbinders' red cloth may be used instead of glass and paper. In whatever way the dark room is lighted, the plate must be shaded from it as much as possible, and only brought into the full light for any length of time when development is nearly complete, and it is necessary to use all safe light to watch the completion of the process. The unpacking and storing of plates, and placing them in the dark slide, may be conducted in almost entire darkness. Before commencing work the following solutions should have been made up:-1

${ }^{1}$ Since this article was originally written, photographic chemistry has made great advances; the above developing (\&c.), solutions are here retained as good, but knowledge of and experience in the art will enable the operator to make choice of the modern formulæ most suitable for his own method of working. 


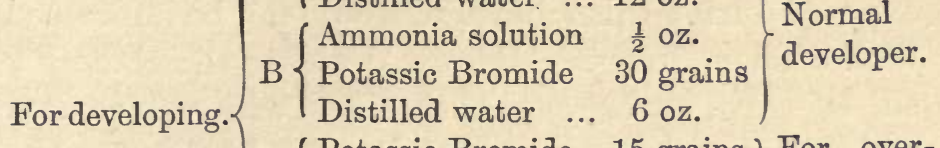
C $\{$ Potassic Bromide 15 grains $\}$ For over-

D $\left\{\begin{array}{ll}\text { Ammonia solution } & 1 \mathrm{drm} . \\ \text { Distilled water } \ldots & 1 \mathrm{oz} .\end{array}\right\} \begin{aligned} & \text { For under- } \\ & \text { exposure. }\end{aligned}$

For hardening. Alum saturated solution in common water.

For fixing. \{ Sodic hyposulphite, 4 oz. $\{$ Common water, 1 pint.

For intensifying. $\left\{\begin{array}{c}\text { Mercuric chloride saturated solution in } \\ \text { common water. }\end{array}\right.$ $\left\{\begin{array}{l}\text { Ammonia solution, } 1 \mathrm{oz} . \\ \text { Common water, } 8 \mathrm{oz} \text {. }\end{array}\right.$

Forremoving stains $\{$ Hydrochloric acid, 8 drops. Common water, 4 oz.

A beaker of about two and a half ounces capacity, a drachm measure graduated to minims (60th of a drachm-drops), a papier-maché tray, $4 \frac{1}{4}$ by $3 \frac{1}{4}$, for developing, a porcelain dish to hold the hyposulphite of soda ("hypo"), another dish or tray of papiermaché or porcelain to hold the alum, a broad camelhair brush, and an abundant supply of water should also be in readiness. Operations may then be commenced by taking a plate from the box, and at once replacing the lid, passing the brush gently over its coated surface to remove particles of dust, and placing it in the dark slide, previously dusted inside, which is then to be shut up. Neither in this nor in any other operation must the sensitive surface be touched with the fingers. The slide is then carried to the camera, placed in position, and the exposure given. This, however, must not be done by simply drawing the shutter, for in this act vibrations are 
inevitably set up in the apparatus, and the plate, if exposed during this vibration, would not be impressed with a sharp image. Before drawing the shutter a slip of card, covered on both sides with velvet, and attached to a separate stand, should have been interposed in the beam of light between object and object glass, if the latter is of $1 \frac{1}{2}$ inches or lower power; behind the stage if of higher power. With this stop in position the shutter may be drawn without exposing the plate. A few seconds are then allowed for vibrations to subside; the stop is taken in the hand and held for a second touching nothing, and then quickly removed and the exposure commenced, the time being noted. If the exposure is only to be of a few seconds' duration, the operator still retains the stop in his hand and remains perfectly still while he counts the seconds on a watch, or the vibrations of a ball attached to a string 39 inches long, and held in his other hand. The instant the time expires the stop is replaced, the shutter closed, and the slide withdrawn and carried to the dark room; 2oz. of A solution are then poured into the beaker (there is no need to be particular to half a drachm or so, and it is easy to guess when sufficient has been poured into the beaker). The light is then turned very low, and the operator, turning his back to the lamp, removes the plate, passes the brush over it once more, and places it face upwards in the developing dish; then the "pyro" solution from the beaker is poured on to the plate with a steady and rapid motion, so that the whole plate is flooded at once. The tray is then put aside in a shady place for a few minutes, while the operator measures out 25 drops (minims) of B. 
Here again an excess or deficit of three or four drops is of no consequence. This solution is then to be poured into the beaker, into which also the "pyro" solution from the tray is to be poured, and the mixture is then returned to the tray in an even wave, and kept gently rocking. For a few seconds nothing will be visible, and for that time it is quite as well to shade the plate from the light, then, if the exposure has been correct, gradually the highest lights will appear and get deeper and deeper, and detail will begin to appear in the lightest half tones. If all is going well, the plate may be left at this stage while an additional 50 drops of $\mathrm{B}$ are measured out and poured into the beaker. Again the solution from the tray is to be returned to the beaker, and again the mixture poured back and the tray kept rocking.

By this time the picture should be full of detail, and more light will be required, and may be safely used. This is a most important time, and great judgment is required to know when to stop the development. In the feeble light employed, it will seem as if the picture is blackening all over and disappearing, but really, if properly exposed, it is only gaining density. The judgment will be assisted by removing the plate from the tray, and holding it up before the light; the amount of density can then be better seen. If judged insufficient, the plate is returned to the tray and rocked a few seconds longer. If the exposure has been properly timed, the whole development will occupy about two and a half or three minutes from the first application of the $\mathrm{B}$ solution. When at last the proper moment arrives, the plate is to be removed from the tray, 
and well swilled with water from a jug or tap. It is then to be placed face upwards in the dish of "hypo," and left there five minutes or so, until all trace of milkiness disappears from the back of the plate. It may then be removed from the "hypo," again swilled with water, and if the weather is not hot, and the plates are known to have no tendency to frill, it may be placed in a dish of water and left to soak. This soaking must be continued for about six hours, in several changes of water. The plate may then be stood on end to dry. Heat must not be applied to hasten the drying, or it will cause the gelatine to dissolve. From the time the plate comes from the hypo it is insensitive to light, and, indeed, it may be taken into the light and fixed there as soon as the developer is washed off, without any considerable harm happening to it, but it is best not to expose it to light until it is fixed. The photographer cannot be sure of the character of his negative until it is fixed, and during this process it will undergo a remarkable change, it will lose its translucency and become transparent. If the exposure and development have been correct, the highest lights will be so dark as to make it very difficult to distinguish even bright objects through them, while the deepest shadows should be almost as clear as the plain glass, and the half tones full of detail.

If on the first application of $B$, the details come up with undue rapidity and then begin to fade away, the plate has been over-exposed, and the developer must be instantly poured back into the beaker and the plate flooded with water while 20 to 30 drops of $\mathrm{C}$, according to the amount of over- 
exposure, are measured out and added to the contents of the beaker, which are then to be returned to the plate after the water has been poured off. This will retard the development and preserve the purity of the shadows, while the high lights gain in density. After a time 30 or 40 drops of $\mathrm{B}$ may be added to complete the development. Under-exposure is indicated by slowness in the appearance of detail in the shadows and half tones after the full quantity of $\mathrm{B}$ has been added, and is remedied by the addition of 10 to 20 drops of $\mathrm{D}$ to the developer. In all cases when additions have to be made to the developer, the additional substance should be poured into the beaker, and the solution from the plate added to it. This will secure a uniform mixture before the developer is returned to the plate. If the additions were simply poured directly into the tray they would act locally and spoil the picture. The operator must learn to recognise an under- or an over-exposed plate by its character after development as well as before. Over-exposure results in thin (i.e., weak or transparent), flat, soft negatives, full of detail, but deficient in intensity, while an under-exposed and forced plate yields a dense and harsh negative with little detail. It is useful to know this, because one may occasionally wish to give the negative a soft or a harsh character to suit some special subject. The rule is, for violent contrasts, under-expose and force in development; for a finely-graduated image over-expose, and use the bromide freely. For most purposes we should avoid either extreme.

If the films have a tendency to frill (i.e., to pucker and leave the glass at the edges) or blister 
during or after development-and in very hot weather all plates have these tendencies-they must be soaked for about five minutes in the alum solution, both before and after fixing, to harden the films. In hot weather one should not wait for the commencement of frilling or blistering before applying the remedy, but take it for granted that it will occur, and pass every plate through the alum. It can do no harm in any case.

Plates developed with "pyro" acquire a pale brown or yellow colour, which, if strongly marked, it may be advisable to remove by a few minutes' immersion in the hydrochloric acid solution. Overexposed or under-developed plates can be intensified as follows: The mercuric chloride solution is first applied and kept in motion until the negative becomes a very pale grey, almost white. If the operation be conducted in a black dish the negative will appear as a most beautiful positive when the whitening is complete. The plate should then be thoroughly washed for at least half-an-hour in several changes of water. The weak ammonia solution should then be applied, and in a few seconds will blacken the image. It will then want a thorough washing in several changes of water, prolonged through about six hours, after which it may be stood in a rack to dry.

When dry, the negatives must be varnished with photographic varnish flowed over them while hot. This will protect the films from mechanical injury and from silver stains in printing. When negatives have been intensified, it is especially necessary to varnish them as soon as possible, as they are liable to turn white by exposure to the air. 
It is very difficult to lay down any general rule for length of exposure, since there are so many variables-thickness and colour of the object, colour of the medium (balsam especially sometimes acquires a yellow colour highly objectionable), focal length and angular aperture of the object glasses, and the number of lenses and kind of glass of which they are composed, length of camera, rapidity of plates, presence or absence of eye-piece, and mode of illumination, all have to be taken into account to give some idea of what may be expected; we append particulars of a few of our negatives. The first three were taken with an A eye-piece, and a lamp having a 1 inch wick only. The plate was about 12 inches from the object.

"Tongue" of Blowfly. Balsam. - Two inch objective by Stanley. Home made plate. Five min. Ferrous oxalate developer. Under-exposed.

Diatom. Coscinodiscus Excavata.- $\frac{1}{2}$ inch objective and achromatic condenser. Home made plate. 10 min. Pyro. Correct exposure.

Diatom. Auliscus sculptus. $-\frac{1}{8}$ inch objective by Beck, and achromatic condenser. Edwards' plate. 26 min. Pyro. Rather over-exposed.

The following were taken without an eye-piece, and with a lamp having a $1 \frac{1}{2}$ inch wick. The distance of the plate from the object is given in each case.

Tongue of Cat injected carmine and stained. Transverse Section. Balsam. -3 inch objective of $12^{\circ}$ by

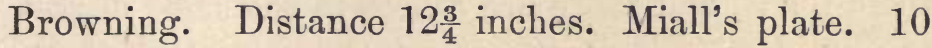
secs. Pyro. Correct exposure.

Rachis of Pteris Aquilina. Transverse Section unstained. Gly. jelly. -2 inch objective by Stanley. 
Distance $22 \frac{1}{2}$ inches. Miall's plate. 1 min. Pyro. Over-exposed.

Human Kidney injected carmine. Transverse Section in Balsam.-Object yellowish. 1 inch objective by Stanley. Distance 36 inches. "German" plate $1 \frac{1}{2} \mathrm{~min}$. Oxalate. Correct exposure.

"T'ongue" of Blowfly. Balsam. $-\frac{1}{4}$ inch objective and achr. condenser. Distance 40 inches. Lancaster's plate. $1 \frac{1}{2}$ min. Oxalate. Correct exposure.

Spicules of Synapta.-Dark ground illumination by spot lens. 1 inch objective by Stanley. Distance 41 inches. "German" plate. 2 min. Oxalate. Correct exposure.

Salicine.-Polarized light. Crossed nicols. Without selenite. 1 inch objective. Distance 20 inches. Home made plate. $2 \frac{1}{2} \mathrm{~min}$. Oxalate. Correct exposure.

Ctenoid scales. Skin of sole. Dry, as opaque object. White, but under cover-glass, and therefore loss of light by reflection. Light condensed as usual, but incident at about $10^{\circ}$. 3 inch objective of $12^{\circ}$ by Browning. Distance 28 inches. Miall's plate. 5 min. Pyro. Correct exposure.

These examples have been selected as illustrative of very various objects, magnifying powers and modes of illumination. Opaque objects are the most difficult, on account of the trouble of focussing by the small amount of light they reflect.

Although any object glass may be used for this work, some possess qualities which specially fit them for it, and there are others in the same degree unsuitable. The most important properties of an objective for photographic work-good definition being, of course, pre-sūpposed as essential for all 
purposes - are penetration and flatness of field. These qualities are of greater importance in lenses for photographic than for visual purposes, for in viewing an object under the microscope the observer has the power of focussing in rapid succession, and by imperceptible gradations, points at different depths and different distances from the centre of the field; but a photograph represents only such structures as were in focus at the time of exposure, and once taken, the focus is unalterable. It is therefore desirable to secure as great a depth of focus and as flat a field as possible-qualities which are incompatible with large apertures.

This brief chapter is merely elementary and intended as a guide to beginners. Those who desire to thoroughly acquire and successfully practise this fascinating art, and to learn the details of the most recent processes and manipulations, as well as the use and value of apochromatic objectives and of all other modern appliances and apparatus, should obtain "Practical Photo-Micrography" recently issued by that master of the art, Mr. Andrew Pringle, and published by Messrs. Iliffe and Son, St. Bride Street, London, E.C. 
In bidding farewell to his readers the author ventures to express the hope that the results of his thirty years' experience in microscopy and microscopical manipulation, as embodied in this treatise, may tend to simplify the work which has lost none of its interest or attractions for him, and that those who may be induced to prosecute this delightful pursuit may be enabled thereby to far surpass any results or achievements upon which he may venture to consider he is entitled to congratulate himself. At least he may affirm that his work has been always prosecuted with the most sincere desire and endeavours to produce the best results and to advance true microscopical science. 


\section{IN DEX.}

A.

ABSOLUte alcohol, hardening tissues by, 49 .

Acetate of potash, 103.

Adenoid tissue, 58.

Alcohol, as a hardening agent, 41; dilute, 54.

Algæ, fluid for mounting, 104 ; to clean diatoms on, 146.

Alum cochineal staining, 86.

Ammonia carmine staining fluid, 82.

Ammonium bichromate, 46.

Anilin blue black, 86 .

Anilin colors as stains, 87 ; list of, 88.

Anilin stainings, clearing, 91.

Animal tissues, preparation of, 38 . Animal, to inject an, 71, 73.

Apparatus,list of, for laboratory, 32.

Aqueous humour of eye, 16.

Aqueous injection fluids, 65 .

Art, microscopical, 7.

Asphalt varnish, 107.

Astigmatism, 21.

\section{B.}

BACILII and bacteria, staining, 91, 92, 93, 94; Rev. Gordon Thompson's process, 94.

Balsam, Canada and xylol, 78.

Beale, Dr., on instruments and their uses, 27 ; on methods of preparation, 6 .
Beetle, muscle of, 49.

Bell's cement, 106.

Bichromate of potass ; 45 .

Binocular vision, 24, 25.

Bismarck brown, 89.

Blood vessels, 57 ; injection of, 61 , 69.

Blue injection mass, 64 ; fluids, $66,67,68$.

Borax-carmine staining fluid, 83.

C.

Camera lucida, in microscopical drawing, 167.

Canada balsam, 99 ; mounting in, 133 ; diatoms in, 149.

Capillary vessels, 61; development of, 59 ; injecting, 69.

Carmine injection fluids, 65, 66 ; mass, 62 ; staining, 78; -fluids, Beale's, 82 ; Huxley's, 83 ; Ranvier's, 82.

Carrot, embedding in, 111.

Cartilage cells, 56.

Cathcart microtome, 127.

Celloidin embedding, 117.

Cement, Bell's, 106; dammar, 105; gelatine, 106; gold size, 107 ; gutta-percha, 107 ; marine glue, 107 ; mastic, 109 ; shellac, 107 ; white zinc, 107.

Cement substance of muscle, 58 .

Cements, 105.

Chemicals for laboratory, list of, 34. 
Chloride of gold, 59.

Choroid coat of eye, 16-18.

Chromate of ammonium, 46.

Chromic acid, 42; and alcohol, 43 ; and nitric acid, 55.

Chrysoidin, 93.

Ciliated epithelium, 54.

Clearing media, 95; (on) and manipulation of sections, 96 .

Clearing with xylol and phenol, 95 ; anilin stainings, 91 ; clove oil, 97 ; bergamot oil, 98 ; cedar wood oil, 98 ; turpentine, 97.

Cochineal staining fluid, 86; alum, 86.

Colour, 23.

Colour-blindness, 23.

Colours for use in microscopical drawing, 177, 179.

Conversion of French fluid measures into English ditto, 36; weights, 37.

Cornea of eye, 15, 57, 59.

Crystalline lens of eye, 1, 16, 17.

\section{D.}

Dammar varnish, 105.

Deane's (mounting) medium, 101.

Developing fluids for photo-micrography, 192.

Developing photo-micrographs, 194.

Development of capillaries, 59 .

Diatomaceæ, the preparation of, 144 ; to clean, 146, 147; to mount in balsam, 149 ; to mount "dry," 148; mounting stage for, 152 ; to mount in styrax, 157.

Double staining vegetable sections, 142 ; with hæmatoxylin and eosin, 89; with picro-carmine, 144.

Drawing and painting (microscopical), 160.

'Dry " slides, 148.
E.

EHRLICH's hæmatoxylin staining fluid, 79; staining for bacilli, 93.

Embedding, 109; in carrot, 111 ; celloidin, 117; gum and glycerine, 116; paraffin, 113 ; pith, 112 ; wax and oil, 113.

Endothelium, 56, 57.

Eosin, 89.

Epithelium, 5.

Examples in photo-micrography, 199.

Eye, the human, essay by Geo. E. Davis, F.R.M.S., F.L.S., \&c., 13; aqueous humour of, 16 ; choroid coat of, 16,18 ; cornea of, 15 ; crystalline lens of, 1,16 , 17 ; imperfections in, 14 ; iris of, 15,17 ; pigmentum nigrum of, 16 ; retina of, 16,18 ; sclerotic coat of, 15 ; vitreous humour of, 16.

F.

Farrant's medium, 102 ; mounting in, 137.

Fearnley, Dr., injecting apparatus, 73,74 ; freezing microtome, 126.

Fill an ice microtome, to, 125.

Fluid measures, French, conversion into English ditto, 36.

Fossil deposits, to clean, 147 ; dia. tomaceæ, 147.

Freezing, 109.

Frog, 56, 58, 60.

Fuchsin and eosin, 87.

G.

GAGE's picro-carmine staining fluid, 84 .

Gelatine, 61 ; cement, 106; and honey, 101.

Gibbes', Dr. Heneage, stain for bacilli, 92,

Glycerine, 53, 102 ; jelly, 100; (mounting in) 136; Kaiser's, 101; Lawrence's, 101; mounting in, 135. 
Goadby's fluid, 104.

Gold chloride, 59.

Gold size, 107.

Green injection mass, 65.

Gum and glycerine, 102 ; (embedding), 116.

Gum and syrup, 118.

Gum styrax, 105.

Gutta-percha cement, 107.

H.

HexaTOXYLIN staining fluids, 79, 80; Ehrlich's,79; Kleinenberg's, 81; Mitchell's, 81; Weigert's, 80.

Hæmatoxylin and eosin, double staining, 89.

Hardening agents, 38 ; tissues, 38 ; by absolute alcohol, 49 ; by alcohol, 41; bichromate of potass, 45; ammonia, 46; chromic acid, 42 ; and alcohol, 43; chromate of ammonium, 46; Müller's fluid, 47; and spirit, 48 ; picric-acid, 50 ; osmic acid; 51 .

Higginson's syringe, 75, 76.

\section{I.}

ICE microtome, to fill an, 125.

Illumination in microscopical drawing, 171; in photo-micrography, 188.

Injecting, methods of, 69 ; an animal, 71; by Dr. Fearnley's apparatus, 73,74 .

Injection of blood vessels, 61, 69; with syringe, 69 ; capillaries, 69 .

Injection fluids, aqueous, 65 ; blue, $66,67,68$; carmine, 65,66 .

Injection mass, 61; blue, 64; (Robin's), 64 ; carmine, Carter's, 62 ; Stirling's, 62; Sims Woodhead's, 63 ; green, Scheeles', 65 ; red, 61 .

Injecting of various organs, 69, 70 .
Instruments and their uses, 1, 10, 27 ; Dr. Beale, on, 27.

Introduction, 1.

Iodine green, 90.

Iris of eye, 15, 17.

K.

KAISER's glycerine jelly, 101.

Kleinenberg's hæmatoxylin stain, 81.

L.

LABORATORY, list of apparatus for, 32 ; of chemicals for, 34 .

Lacteal, injection of, 49 .

Lawrence's glycerine jelly, 101.

List of anilin colours for staining, 88; apparatus required, 32 ; chemicals, 34 .

Logwood staining, 78.

Lungs, 57.

Lymphatic gland, 49; vessels, 61. Lymphatics, 58.

M.

Margarine crystals, 53.

Mastic cement, 109.

Membranous connective tissue, 58.

Methods of preparation, 5; Dr. Beale on, 6.

Methyl green, 90 ; violet, 90 .

Micro-photography, 8.

Microscope, the, 10; for work, 28; objectives for, 31 ; in microphotography, 183.

Microscopical art, 7.

Microscopical drawing and painting, 161; camera lucida in, 167; colours for, 177, 179; illumination in, 171 ; paper for, 177.

Microtomes, 119.

Microtome, Cathcart's, 127 ;

Fearnley's, 126; Minot's, 129 ;

Rutherford's, 121; Thoma, 129;

Williams', 123.

Minot's microtome, 129. 
Miscellaneous preparations, 53.

Mitchell's logwood stain, 81.

Monobromide of napthalin, 105.

Mounting (on), 132; in Canada balsam, 133; Farrant's medium, 136 ; glycerine, 135 ; glycerine jelly, 136 ; diatoms, 148, 149 ; insects, 138; polycystina and radiolaria, 157.

Mounting media, 98 ; acetate of potass, 103; Canada balsam, 99; hardening of, 99; and benzol, 99 ; and xylol, 99 ; Deane's fluid, 101; glycerine, 102 ; jelly, 100 ; Goadby's fluid, 104 ; monobromide of naphthalin, 105 ; styrax, 105.

Mounting medium for algæ, 104.

Mounting stage (Cole's), 152.

Mouse, 60.

Müller's fluid, 47 ; and spirit, 48. Muscle, 60.

N.

NAPHTHA and creasote, 104.

Nerve ganglia, 60.

Nitrate of silver, 55 .

Nitric acid, 42.

Nuclei (of cells), staining, 77.

\section{O.}

OBJECTIVES for working microscope, 31.

Oil of bergamot, 98 .

Oil of cloves, 97 ; cedar wood oil, 98.

Olfactory epithelium, 54.

Omentum, 56.

Orange and rubin, 91.

Organ of vision in man, constitution of, 1.

Osmic aoid, hardening by, 51 ; its uses, 51.

P.

PAPER for microscopical drawing, 177.

Paraffin, embedding in, 113.
Phenylamin, 93.

Photo-micrographs, developing, 194.

Photo-micrography, on, 180 ; illumination in, 188; developing fluids for, 193 ; examples in, 199.

Picric-acid, hardening by, 50; its uses, 56.

Picro-carmine staining, 78; fluid, Gage's, 84; Weigert's, 84 ; double staining, 144; and eosin, 85.

Picro-lithium carmine, 85.

Pigmentum nigrum of eye, 16.

Pith, embedding in,

Polycystina, cleaning and mounting, 157.

Preparation of animal tissues, 38 ; insects, 138 ; diatomaceæ, 144 ; vegetable sections, 141.

Preservation of vegetable tissues, 131.

Prussian blue injection mass, 64 . Prussian blue staining fluid, 66, 67, 68.

Pseudoscopic vision, 25.

R.

RAdIolaria, on cleaning and mounting, 157.

Rat, 60.

Reagents, constitution and action of, 4 .

Red (injection) mass, 61, 62, 63.

Retina of eye, 16, 18.

Rutherford's microtiome, 121.

S.

Saffranin, 91.

Salt solution, 53.

Sclerotic coat of eye, 15, 18.

Sciatic nerve, 58.

Section cutting, 119.

Septum cysternæ lymphaticæ, 56.

Shellac cement, 107.

'Shells, to clean diatoms growing on, 146. 
Silver nitrate, 55 .

Skin, 60.

Spiller's purple, 91.

Squamous epithelium, 54.

Staining fluids (on), 77 ; anilin, 87 ; anilin blue black, 86 : carmine, 82, 83 ; hæmatoxylin, 79, 80,81 ; picro-carmine, 84,85 ; sulph-indigotate of soda, 85 .

Staining, triple, 87.

Staining (on), 77; with anilin dyes, $88,89,90,91$; bacilli and bacteria, 91, 92, 93; picro-carmine, 144.

Staining and mounting vegetable sections, 143.

Stereoscopic vision, 25.

Striped muscle, 60.

Styrax, 105.

Sulph-indigotate of soda, 85 .

Syringe, injecting with, 71; Higginson's, 75, 76.

T.

TAIL of mouse, 60 ; rat, 60 .

Tendon, 57, 60.

Thoma microtome, the, 129.

Thompson's, Rev. Gordon, process for staining and mounting bacilli, \&c., 94 .

Transitional epithelium, 54.
Triple staining, 87 .

Turpentine, 97; clearing with, 97.

V.

VARNISH, asphalt, 107.

Vegetable tissues, preservation of, 131 ; sections, preparation of, 141 ; staining and mounting, 142.

Vesuvin, 91.

Vision in man, limited, 10.

Vitreous humour of eye, 16.

W.

WATER, 54.

Wax and oil, embedding in, 113.

Weigert's hæmatoxylin staining fluid, 80; picro-carmine, 84; for bacilli, 94 .

Weights, French, conversion into English ditto, 37.

White zinc cement, 107.

Williams' freezing microtome, 123.

Woodhead, Dr. 'Sims, injection mass, 63 .

$\mathrm{X}$.

XrLol balsam, 78, 88, 96.

Xylol and phenol clearing medium, 88,95 . 


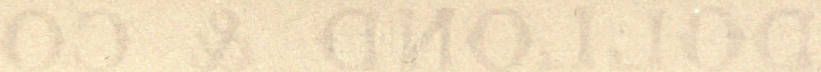
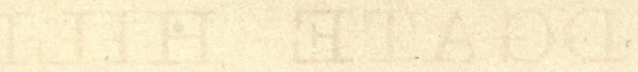

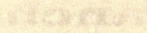

Dinf 


\section{DOLLOND \& $\mathrm{CO}_{x,}$}

35, LUDGATE HILL, And Branches,

SII)anufacturíng Optícians. ESTABLISHED 1750.

MICROSCOPES IN GREAT VARIETY. BAROMETERS, THERMOMETERS,

FIELD AND OPERA GLASSES.

Telescopes for Gea, Sand, or Shl?. PHOTOGRAPHIC APPARATUS.

\section{SPECTACLES, EYE-GLASSES,} And every Requisite for MICROSCOPIC \& PHOTOGRAPHIC WORK Can be supplied by us.

Please Note Address:35, LUDGATE HILL. 


\section{MASON'S MICROSCOPES.}

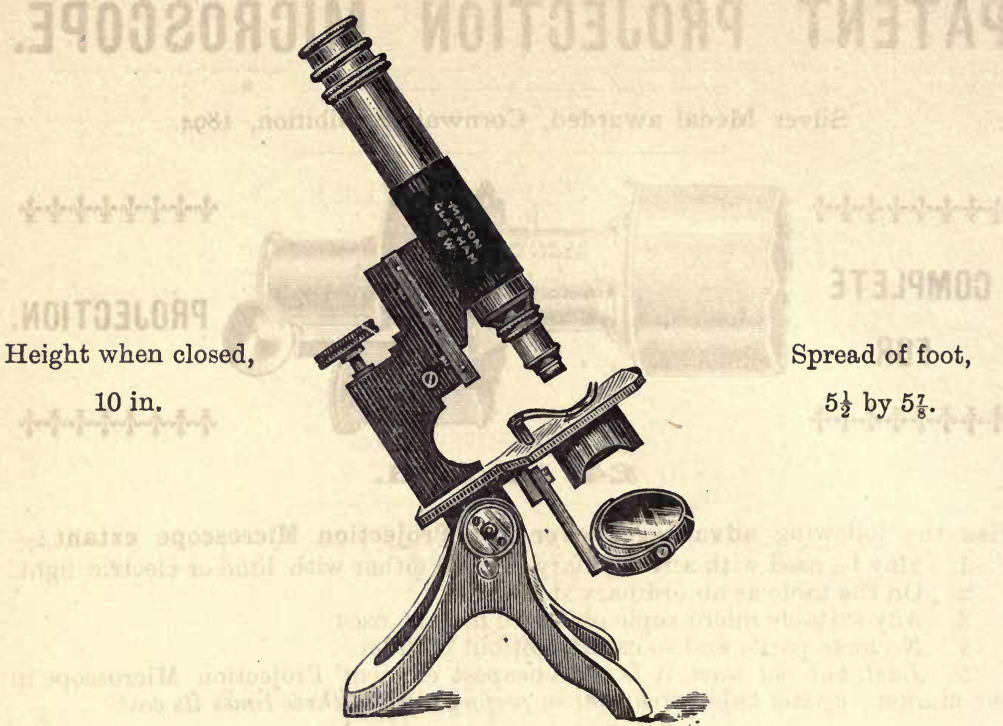

The S.W. Histological and Botanists' Microscope as above Fig. With 1 in. $23^{\circ}$ and $\frac{1}{6}$ th $80^{\circ}$ in Polished Mahogany Case with 1 B Eye-piece. £4 17s. 6d.

This instrument is confidently recommended to Students as thoroughly reliable. The fine Motion is equal to the most expensive, Coarse ditto slides through velvet-lined fitting ensuring a perfectly smooth motion. All fittings are compensated for wear, making it the cheapest and most efficient Microscope ever offered at the price.

$$
\text { R. G. MASOI }
$$

(From J. SWIFT),

69, PARK ROAD, CLAPHAM, LONDON, S.W. ESTABLISHED 1880.

\section{Living Specimens for the Microscope.}

\section{JOHN HOOD, F.R.M.S.,}

REGS to intimate to Teachers and Students of Biology, and those studying B Natural History with the aid of the Microscope, that he has always at hand the following list of Pond Life:-

Protophyta, Protozoa, Rotifera, hydrozoa, Polyzoa, \&c.

Specimen Tube, with drawings and descriptions, 1s. post free. A Series of 26 Tubes, 21s.

Address:-50, DALLFIELD WALK, DUNDEE. 


\section{MASONTS}

\section{PATENT PROJECTION MICROSGOPE.}

Silver Medal awarded, Cornwall Exhibition, I894.

mpmpqumpqum

COMPLETE

FOR

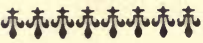

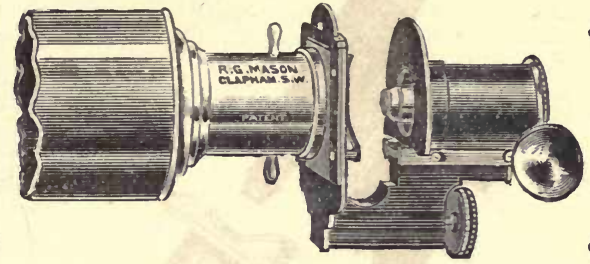

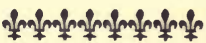

PROJECTION.

24 17s. 6a.

Has the following adyantages oxer any Projection Microscope extant:-

1. May be used with any ordinary lantern either with lime or electric light.

2. On the table as an ordinary stand.

3. Any suitable microscopic objective may be used.

4. No loose parts, and so cannot get out of order.

5. Last, but not least, it is the cheapest efficient Projection Microscope in the market; equals any instrument in performance at three times its cost.

As used by the School Board and Plymouth Technical Schools.

ILLUSTRATED CATALOGUE FOR STAMP.

ALL APPARATUS FOR PHOTO-MICROGRAPHY.

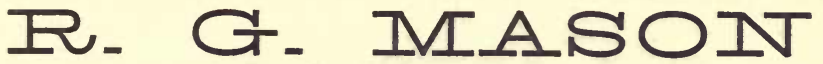

(From J. SWIFT),

69, PARK ROAD, CLAPHAM, LONDON, S.W.

Established 1880 .

\section{MICROSCOPE-Stanley's New Histological,}

With first-class $\frac{1}{6}$ th and $\frac{2}{3}$ rd Object. Glasses, B Eyepiece, Indicator, \&c., in polished Mahogany Case, £5 5s. Improved Complete MOUNTING CABINET, for Practical Physiology, £1 5s. Specially Thin 3 by 1 GLASS SLIPS, 4s. per Gross. Thin Glass Covers, Labels, and all the Necessary Requisites for Students' use, at most Moderate Prices. A large and well-assorted Stock of Special Objects for the microscope, 1s, each. Price List Post Free.

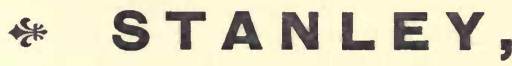

Optician to H.M. Government, Science and Art Department, Council of India, Admiralty, \&c. ; Guy's, St. Bartholomew's, St. Thomas's, and other Hospitals.

CLINICAL THERMOMETERS, with Indestructible Index, in German Silver Cases, 3s. 3d. each.

\section{Railyay Approach, London Briage, S.E.}


C. BAKER'S

OPTICAL AND SURGICAL INSTRUMENT WAREHOUSE, 244, HIGH HOLBORN, LONDON.

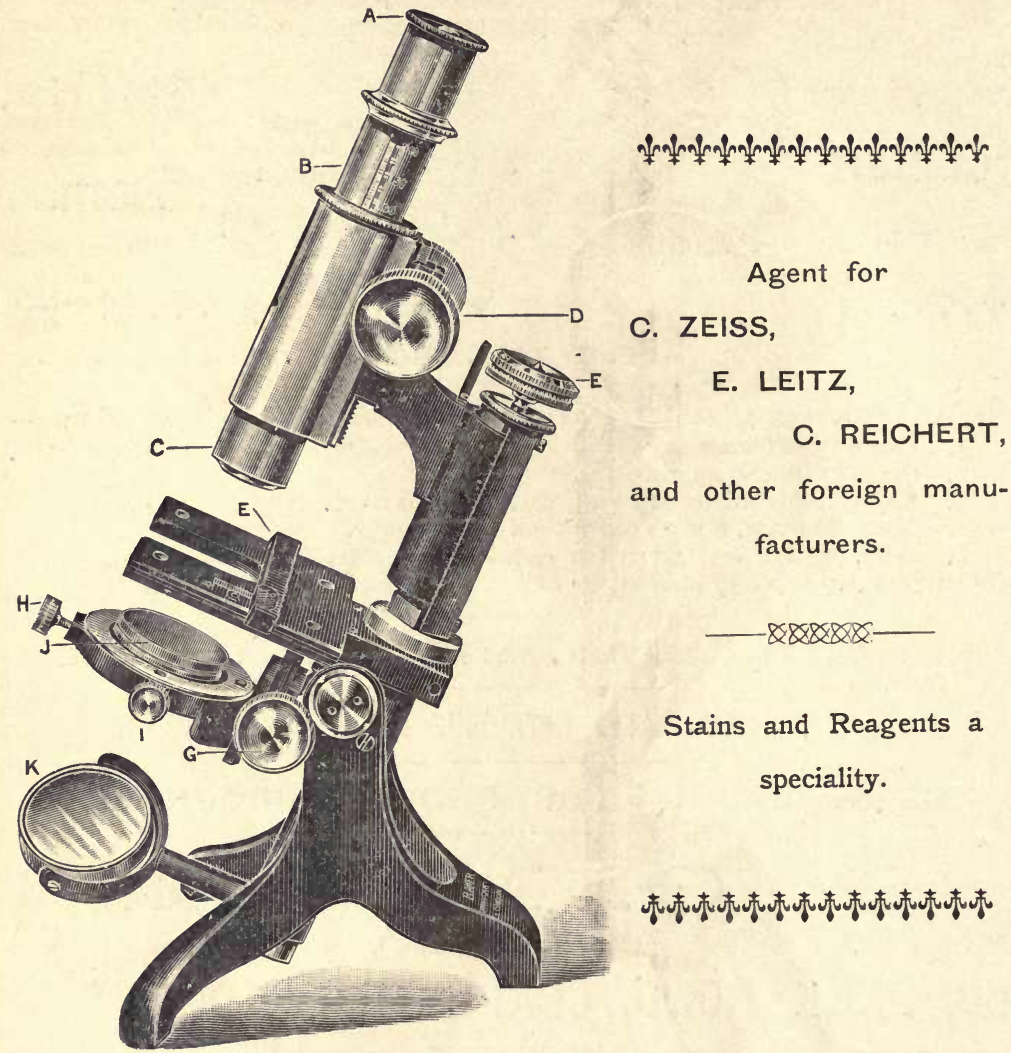

A LARGE COLLECTION OF STUDENTS' MICROSCOPES.

C. BAKER'S MODEL HISTOLOGICAL MICROSCOPE, as figured, with 1 Eye-piece, 2 Objectives by Leitz, No. 3 ( $\frac{2}{3}$ in.) and No. 7

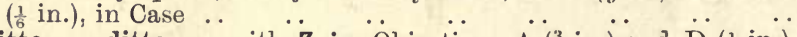

Ditto, ditto, with Zeiss Objectives, $\mathrm{A}\left(\frac{3}{4}\right.$ in.) and $\mathrm{D}\left(\frac{1}{6}\right.$ in.),

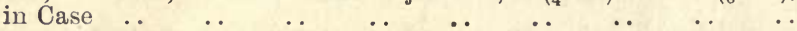
ZEISS'S STUDENT'S MICROSCOPE, with Eye-piece, 2 Objectives, $A\left(\frac{3}{4}\right.$ in.) and D ( $\frac{1}{6}$ in.), in Case .. . . . . .

LEITZ'S STUDENT'S MICROSCOPE, with 2 Eye-pieces, 2 Objectives, No. $3\left(\frac{2}{3}\right.$ in.) and No. $7\left(\frac{1}{6}\right.$ in. $)$, in Case ‥ £3 12s. 6d.,

BACTERIOLOGICAL MICROSCOPES, with 2 Eye-pieces, 3 Objec$£ 6100$ $\begin{array}{lll}£ & 0 & 0\end{array}$ £6 130 \&6 55 tives $\left(\frac{2}{3}\right.$ in., $\frac{2}{6}$ in., and $\frac{\lambda}{12}$ in. Oil), Abbé Condenser, Triple Nose-

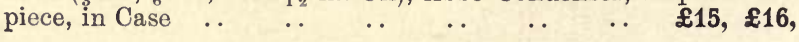

\section{CATALOGUES POST FREE.}




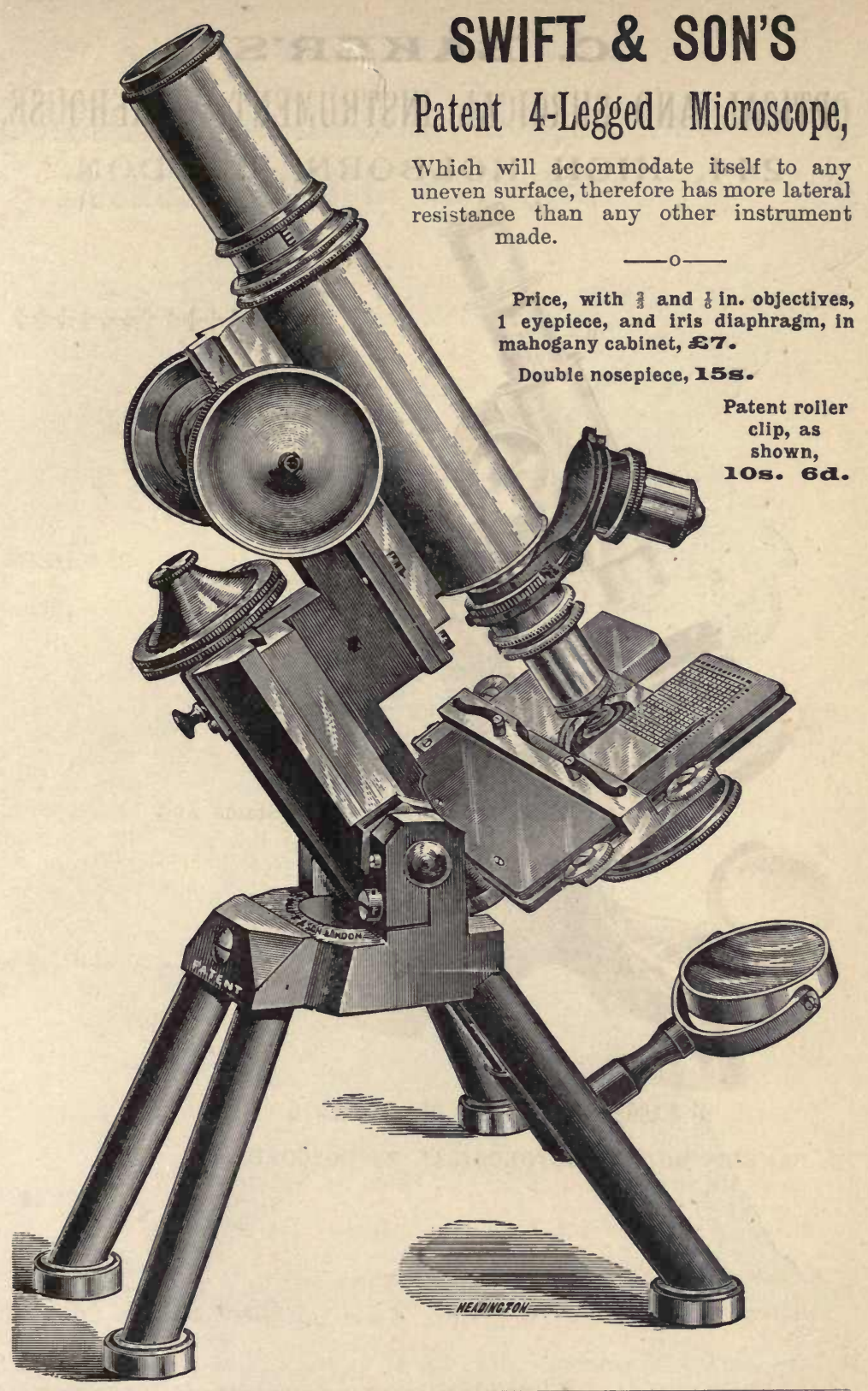

Fully Illustrated Circular on application.

\section{UNIVERSITY OPTICAL WORKS,} 81, TOTTENHAM COURT ROAD, W. 


\section{BAKER'S}

\section{Optical and Surgical Instrument Warehouse, \\ 244, HIGH HOLBORN, LONDON.}

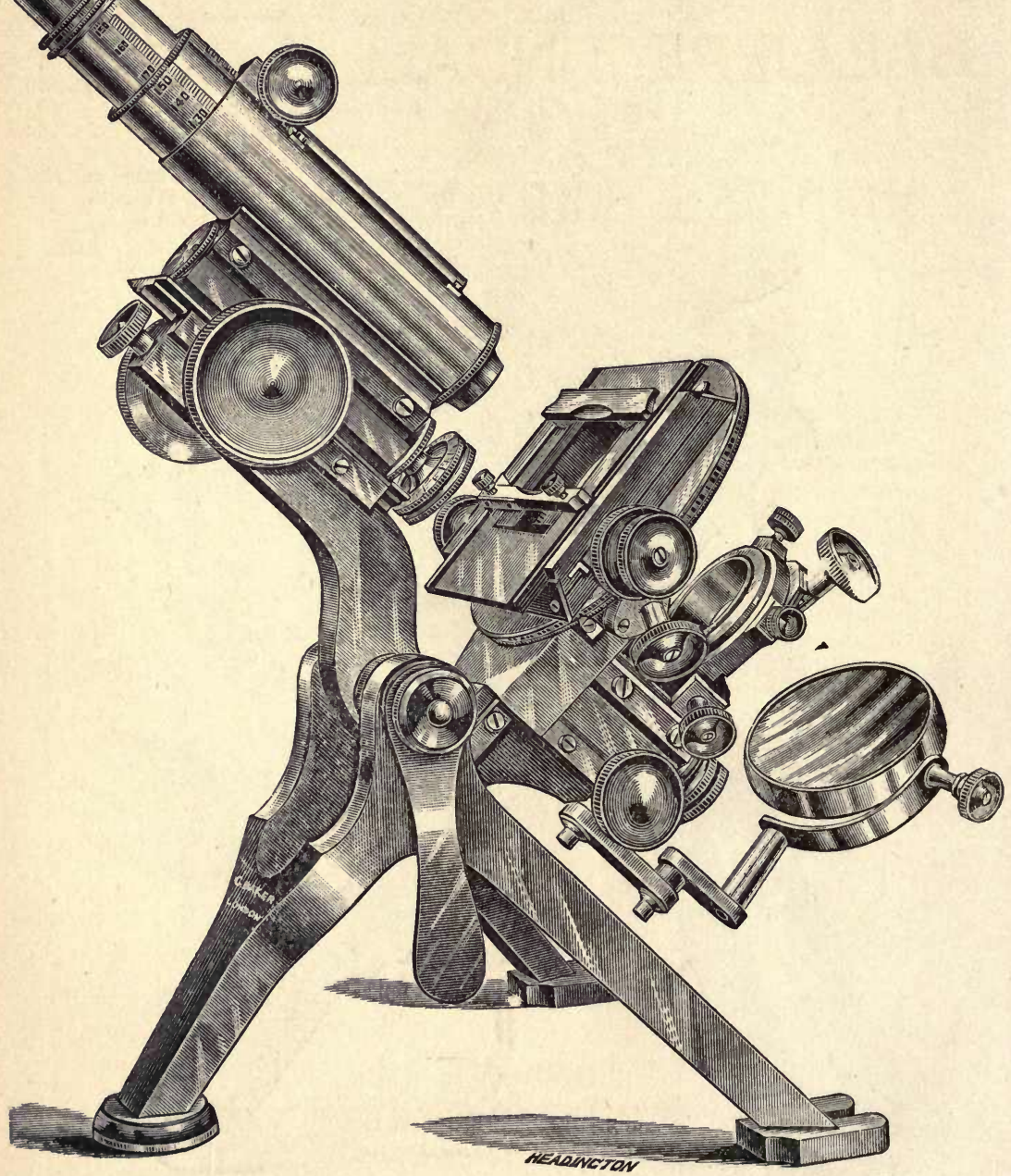

Nelson Model Microscope, No. 1a, as figure ... $\quad \ldots \quad \begin{array}{llll}£ 35 & 0 & 0\end{array}$ $\begin{array}{lllllllllll}\text { Ditto } & \ldots & \ldots & \ldots & \ldots & \ldots & \ldots & \text { No. } 1 & £ 30 & 0 & 0\end{array}$ $\begin{array}{llllllllllll}\text { Ditto } & \ldots & \ldots & \ldots & \ldots & \ldots & \ldots & , & 2 & £ 16 & 16 & 0\end{array}$ $\begin{array}{llllllllllll}\text { Ditto } & \ldots & \ldots & \ldots & \ldots & \ldots & \ldots & , & 3 & £ 8 & 8 & 0\end{array}$ 
A

\section{CATALOGUE OF THE PUBLICATIONS}

or

\section{BAILLIÈRE, TINDALL, \& COX,}

IN

\section{MEDICINE, SCIENCE AND ART.}

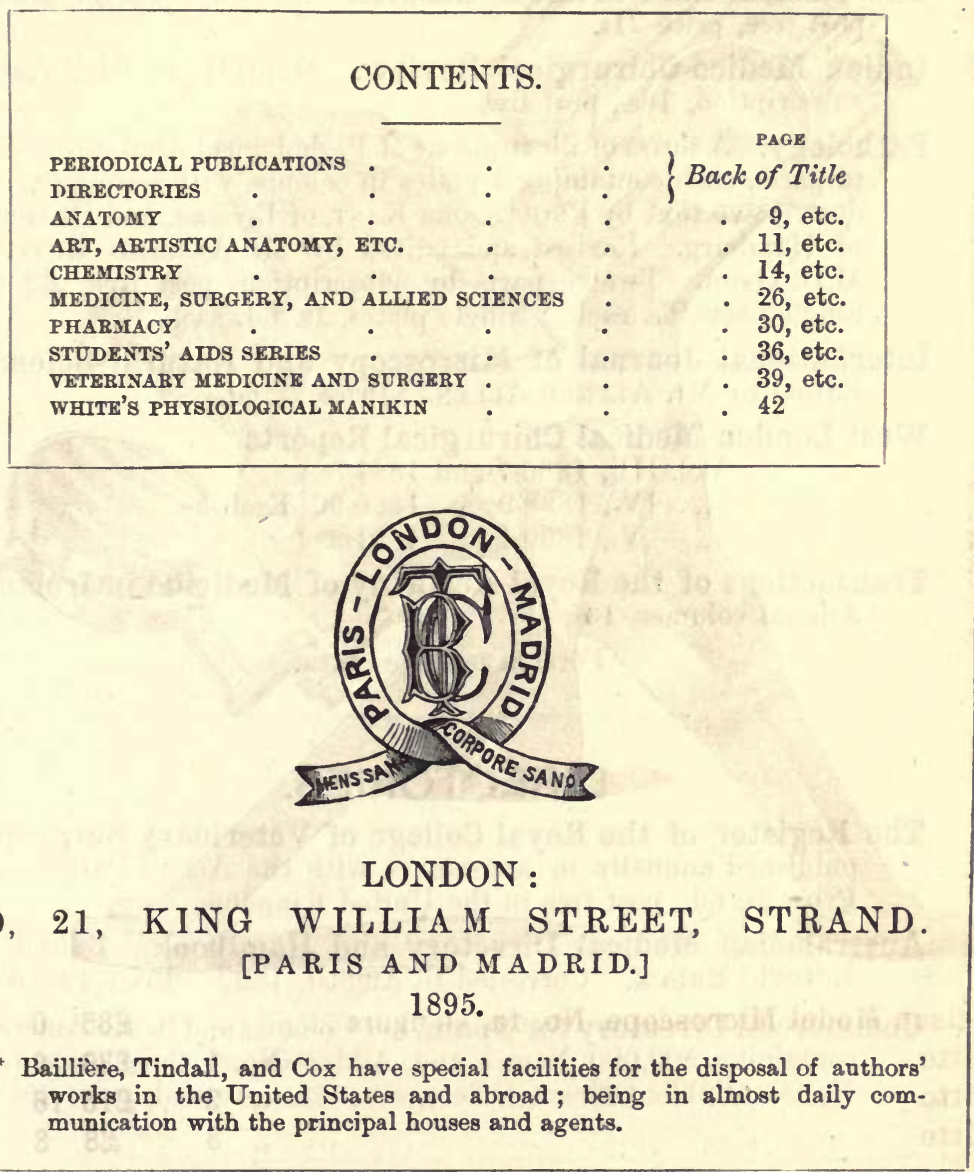




\section{PERIODICAL PUBLICATIONS.}

The Medical Press and Circular. Established 1838. Published every Wednesday in London, Dublin, and Edinburgh. Price 5 d. ; $£ 11$ s. per annum, post free, in advance.

Journal of the British Dental Association. A Monthly Review of Dental Surgery. Published on the 15th of each month. Price $6 \mathrm{~d}$., or $7 \mathrm{~s}$. per annum, post free.

The Analyst. The Official Organ of "The Society of Public Analysts." Monthly, price 1s. ; 10s. 6d. per annum.

The Veterinary Journal, and Annals of Comparative Pathology. Monthly, price 1s. 6d.; 18s. per annum.

The Australasian Medical Gazette. Monthly, 2s., or yearly post free, price $21 \mathrm{~s}$.

Indian Medico-Chirurgical Review. Monthly, 1s. 6d. ; yearly subscription, $16 \mathrm{~s}$., post free.

Pathology. A series of illustrations of Pathological Anatomy issued in parts, each containing 4 plates in colours, with accompanying descriptive text by Profesisons Kast, of Breslau, and RUMPEL, of Hamburg. Revised and edited by M. ARMAND RUFFER, M.D. Oxon. Twelve parts by subscription, post free, £2 8s. Single parts, 6s. each. Single plates, 1s. 6d. each.

International Journal of Microscopy and Natural Science. Edited by Mr. Alfred Allen. Price 2s. 6d.

West London Medical Chirurgical Reports.

$$
\left.\begin{array}{c}
\text { Vol. III., 1886-7 and 1887-8 } \\
" \text { IV., 1888-9 ", 1889-90 } \\
" \text { V., 1890-1 } " 1891-2
\end{array}\right\} \text { Each 5s. }
$$

Transactions of the Royal Academy of Medicine in Ireland. Annual volumes, 14s.

$$
\text { Foreign postage extra. }
$$

\section{DIRECTORIES.}

The Register of the Royal College of Veterinary Surgeons ; published annually in accordance with the Act of Parliament. Price 2s. 6d., post free in the United Kingdom.

Australasian Medical Directory and Handbook. Edited by LUdWIG BRuck. Corrected to August, 1892. Price 12s. 6d.

Commercial Directory for Spain, her Colonies and South America, containing 500,000 Names and Addresses of the Commercial Houses, Public Officers, Offices, etc., etc. Annual, price 25s. 


\section{ALPHABETICAL INDEX OF AUTHORS.}

ABERCROMBIE (J.) On Tetany in Young Children ....................... I 5

ADAMS (W.) Surgical Treatment of Deformities ........................... I7

ALLAN (F. J.) Aids to Sanitary Science .................................... 31

ALLAN (J. H.) Tables of Doses ........................................ 25

ALLEN (Alfred) Microscopical Science ..................................... wrapper

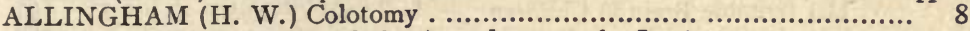

ATTENDANTS. Handbook for Attendants on the Insane ................ 24

BAKER (Benson) How to Feed an Infant ............................... 28

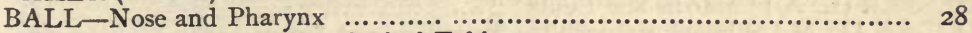

BANHAM - Veterinary Posological Tables ...................................... 39

BANNATYNE (A.) Aids to Pathology ....................................... 29

BEACH (Fletcher) Psychological Medicine ................................. $3 \mathbf{I}$

BERNARD (Claude) and HUETTE'S Text-book of Operative Surgery ....... 33

BLACK (C). Atlas of the Male Organs of Generation .......................... Io

BLACKLEY (C. H.) Hay Fever, its Causes and Treatment ................. 22

BODDY (E. M.) History of Salt....................................... 32

Hydropathy .................................................. 23

BOWDICH (Mrs.) Confidential Chats with Mothers ....................... I5

BOWLES (R. L.) On Stertor and Apoplexy ................................ II

BOYD (Stanley) Movable Atlas of the Foot, its Bones and Muscles ........... 21

BRAND (A. T.) Pocket Case Book ......................................... I4

BROCHARD (J.) Practical Guide for the Young Mother.. ..................... 28

BROWN (George) The Student's Case-book ................................. I4

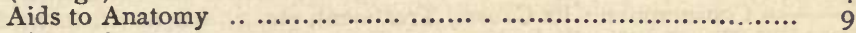

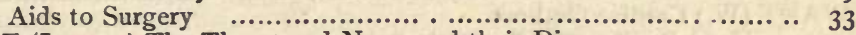

BROWNE (Lennox) The Throat and Nose, and their Diseases ............ 34

Diph theria ................................................... 8

BROWNE (W. J.) The Moon, its Influence on Weather ....................... 27

BURTON (J. E.) Translation of Ebstein's Gout ........................... 22

CAMERON (Chas.) Microbes in Fermentation, Putrefaction, and Disease ... 12

The Cholera Microbe and How to Meet It ...................... I5

CAMERON (Sir C. A.) History of the Royal College of Surgeons in Ireland 23

CAMPBELL (C. M.) Skin Diseases of Infancy and Early Life ................ $3^{2}$

CANTLIE (Jas.) Atlas of the Hand ......................................... 10

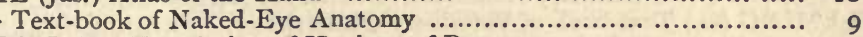

CARDWELL (B.) Translation of Hygiene of Beauty ........................ 24

CASSELLS (J. Patterson) Deaf-mutism and the Education of the Deaf-mute 17

CHARCOT (J. M.) Bright's Disease of the Kidneys............................ 25

CHRISTY (T.) Dictionary of Materia Medica …......................... 25

CHURCHILL (Fleetwood) Obstetrical and Gynæcological Nursing........... 28

CLARKE (J. Jackson) Cancer, Sarcoma and other Morbid Growths .......... I4

CLARKE (Percy) Medical Laws ........................................... 26

CLARKE (E. H.) The Building of a Brain .............................. 13

CLARKE (Ernest) Atlas of Eye ............................................ 20

COFFIN (R. J. Maitland) Obstetrics ..................................... 28

COLE (M. J.) Modern Microscopy ...................................... 27

COOMBE (Russell) Epitome of B. P.................................. 30

COOPER (R. T.) On Vascular Deafness ................................... I8

COTTERELL (Ed.) The Pocket Gray, or Anatumist's Vade Mecum ......... 9

COURTEN.AY (E.) Practice of Veterinary Medicine ........................ 39

COZZOLINO (V.) The Hygiene of the Ear ................................... I 8

CRAWFORD (W. S.) Ulcers and their Treatment ........................... 35

CROOKE (G. F.) The Pathology of Tuberculosis ........................... I 7

CROSS (M. J.) Modern Microscupy ........................................ 27

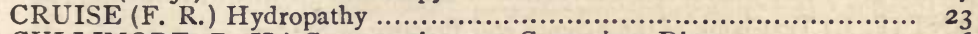

CULLIMORE (D. H.) Consumption as a Contagious Disease ................ 16 
CULLIMORE (D. H.) The Book of Climates..

DARLING (W.) Anatomography, or Graphic Anatomy .....................

DAWSON (W. E.) Guide to the Examinations of the Apothecaries' Society

DAY (W. H.) Irritable Brain in Children ..................................

DENNIS (Hy. J.) Second-Grade Perspective Drawing ....................... Third-Grade Perspective Drawing

DESSAR (L. A.) Catarrhs and Colds

DOLAN (T. M.) Whooping Cough, its Pathology and Treatment.............

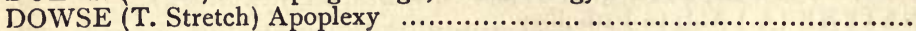

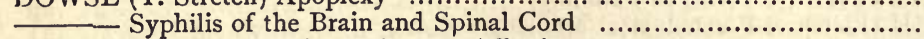

Sy

The Brain and the Nerves and Influenza

DRAGENDORFF (Prof. G.) Plant Analysis

DRYSDALE (C. R.) Nature and Treatment of Syphilis .

DRYSDALE (John) The Protoplasmic Theory of Life.

DUDGEON (R. E.) The Sphygmograph

DUFFEY (G. F.) Note-taking

EBSTEIN (Prof.) The Treatment of Gout

EDWARDS (F. Swinford) Urinary Surgery

ERSKINE (J.) Hygiene of the Ear .............................................

EVANS (C. W. De Lacy) How to Prolong Life?

Consumption : its Causes, Treatment, etc.................

EWART (W.) Cardiac Outlines

Heart-Studies, Chiefly Clinical .

How to Feel the Pulse

Symptoms and Physical Signs

y Clinical .............................................

FAU (J.) Artistic Anatomy of the Human Body .................................

Anatomy of the External Form of Man ................................ I

FIELD (G. P.) Diseases of the Ear .............................................. 18

FINNY (F. M.) Clinical Fever Chart ......................................... 21

FITZGERALD (H. P.) Dictionary of British Plants and Flowers ............. I3

FLAXMAN (J.) Elementary Anatomical Studies for Artists .................. II

FLEMING (G.) Text-book of Veterinary Obstetrics ......................... 39

Neumann's Parasites of Domestic Animals ......................... 39

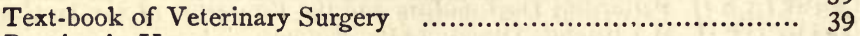

Roaring in Horses ................................................ 40

Practical Horse-Shoeing ......................................... 40

Animal Plagues, their History, Nature and Treatment ............. 40

Contagious Diseases of Animals ..................................... 40

Tuberculosis..... ....................................................... 40

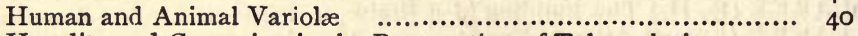

Heredity and Contagion in the Propagation of Tuberculosis ........ 40

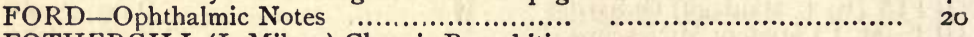

FOTHERGILL (J. Milner) Chronic Bronchitis ….......................... 13 The Physiological Factor in Diagnosis ........................... I 7

Aids to Diagnosis ......................................................... I8

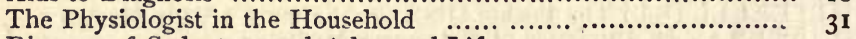

Diseases of Sedentary and Advanced Life .......................... 29

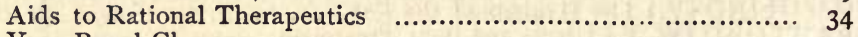

Vaso-Renal Changes ................................................ 24

FOY (Geo.) Anæsthetics : Ancient and Modern ................................. 9

FUCHS (Dr.) The Causes and Prevention of Blindness ........................ 20

GANT (F. J.) Text-book of the Science and Practice of Surgery $\quad . . . \ldots \ldots \ldots . .33$ 


\section{Baillière, Tindall, and Cox's Books.}

GANT (F. J.) Diseases of the Bladder, Prostate Gland,

Examinations by the Conjoint Board ............................... I9

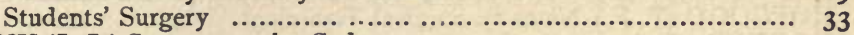

GARMANY (J. J.) Surgery on the Cadaver ................................. 33

GARROD (A. E.) Handbook of Medical Pathology ......................... 29

GEMMELL (Wm.) Dermic Memoranda ....................................... 32

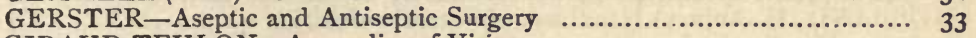

GIRAUD-TEULON-Anomalies of Vision ................................ 20

GLASGOW-PATTESON (R.) Skin and Hair .................................. 32

GOODALL (E.) Microscopical Examinations of the Brain ................... 23

GORDON (Chas. A.) Our Trip to Burmah ................................... I3

Aids to Psychological Medicine ....................................... $3^{8}$

Life on the Gold Coast ............................................... 8

Lessons in Military Hygiene and Surgery ......................... 23

A Manual of Sanitation ............................................. 23

Rabies and Hydrophobia ............................................... 23

Island of Madeira ...................................................... 16

GORDON (T. Hurd) Aids to Practical Chemistry …........................ 36

GORE (Albert A.) Our Services Under the Crown ........................... 27

Medical History of African Campaigns .................................

GOULD -Illustrated Dictionary of Medicine, Biology, etc......................... I8

GOW (W. J.) Handbook of Medical Pathology............................... 29

GRANVILLE (Mortimer) Gout ........................................... 22

GREEN (F. W. Edridge) Memory ............................................ 27

Detection of Colour Blindness............................................. 20

GREENWOOD (J.) Laws Affecting Medical Men ............................. 26

GREEN WOOD (Major) Aids to Zoology …............................ 35

GRESSWELL (J. B. and A. G.) Manual of Equine Medicine and other works 40

GREVILLE (H. Leicester) Student's Hand-book of Chemistry............... 15

GRIFFITHS (A. B.) Micro-Organisms .................................. 12

GRIFFITHS (W. H.) Text-book of Materia Medica and Pharmacy ........ 25

Notes for Pharmacopœial Preparation................................. 30

Posological Tables................................................ 3 I

GUBB (Alfred S.) Aids to Gynæcology .......................................... 22

GUILLEMARD (F. H. H.) Endemic Hæmaturia ........................... 20

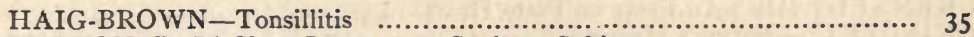

HALTON (R. J.) Short Lectures on Sanitary Subjects .................... 24

HANDBOOK for Attendants on the Insane ................................. 28

HARRIS (Vincent) Manual for the Physiological Laboratory .................. 23

HARRIS (V. D.) Kühne's Guide to the Demonstration of Bacteria ............ 12

HARTMANN (Prof.) On Deaf-mutism, Translation by Dr. Cassells........... I 7

HAYNES (Stanley) Healthy Homes ........................................ 24

HAZARD (W. P.) Diseases of Live Stock .................................... $4 \mathbf{I}$

HEIBERG (Jacob) Atlas of Cutaneous Nerve Supply ......................... 27

HEPPEL - Analytical Conic Sections .............................................. $\quad 38$

HERRINGHAM (W. P.) Handbook of Medical Pathology .................. 29

HERSCHELL (Geo.) Indigestion ........................................... 24

Heart Diagrams and Case-book ............................... 22

HEWITT (Frederic) Anæsthetics .............................................. 9

HILL (J. W.) Principles and Practice of Bovine Medicine ...................... 40

Management and Diseases of the Dog ............................ 40

HIME (T. W.) Cholera : How to Prevent and Resist It... ... ............... I5

The Practical Guide to the Public Health Acts ....................... $3 \mathbf{1}$

HOARE-Veterinary Therapeutics ........................................... 40

HOGG (Jabez) The Cure of Cataract ......................................... 20 
HOGG (Jabez) The Impairment of Vision from Shock PAGE

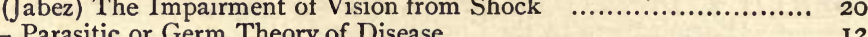

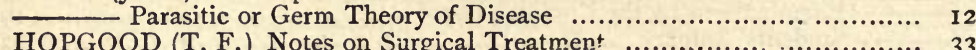

HORNER (Professor) On Spectacles .......................................... 20

HOWAT (G. R.) How to Prevent and Treat Consumption .................... I6

HUNTER (Ch.) Manual for Dental Laboratory ............................... I 7

HUSBAND (H. Aubrey) Handbook of Forensic Medicine .................... 2 I

- Handbook of the Practice of Medicine............................. 26

- Student's Pocket Prescriber ....................................... $3 \mathbf{I}$

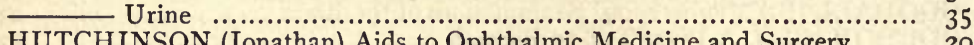

HUTCHINSON (Jonathan) Aids to Ophthalmic Medicine and Surgery ...... 20

ILLINGWORTH-Hydrophobia ..................................... 23

INCE (J.) Latin Grammar of Pharmacy ................................ 30

INTERNATIONAL MEDICAL CONGRESS ............................ 24

JAMES (Brindley) Replies to Questions in Therapeutics ...................... $3^{8}$

JAMES (M. P.) Therapeutics of the Respiratory Passages ..................... 34

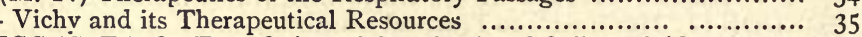

JENNINGS (C. E.) On Transfusion of the Blood and Saline Fluids ............ 35

Cancer and its Complications .................................. I4

JENNINGS (Oscar) On the Cure of the Morphia Habit......................... 27

JESSETT (F. B.) Surgical Diseases of Stomach and Intestines ................ 8

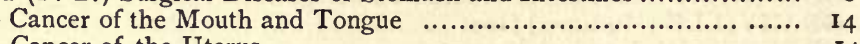

Cancer of the Uterus.....................................................

JONES (H. Macnaughton) The Diseases of Women ......................... 22

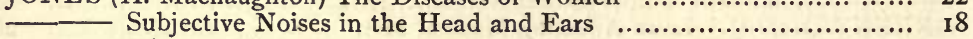

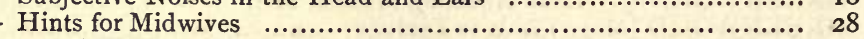

Pharynx................................................................. 19

JONES (H.) Guide to Sanitary Science Exams. ................................. 32

JONES (T. Wharton) Blood in Inflammation ................................ 24

JUKES-BROWNE (A. J.) Palæontology (in Penning's Field Geology) ....... 21

KAST AND RUMPEL_Illustrations of Pathological Anatomy ............. 29

KEETLEY (C.R. B.) Guide to the Medical Profession.......................... 26

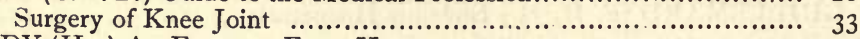

KENNEDY (Hy.) An Essay on Fatty Heart............................. 22

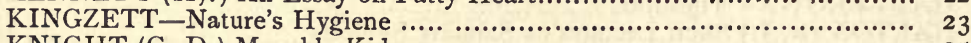

KNIGHT (G. D. ) Movable Kidney ......................................... 25

KUHNE-Demonstration of Bacteria ......................................... I2

LAMBERT (J.) The Germ Theory of Disease ............................. 40

LEASK (J. G.) Questions at Medical Science Examinations .................... I9

LEDWICHI (J.) Ánatomy of Inguinal and Femoral Regions ................. 9

LEONARD (H.) The Pocket Anatomist ....................................... 9

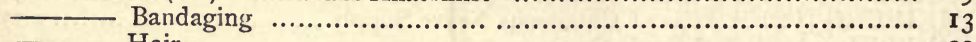

- Hair .................................................................. 22

LE SUEUR CHRISTY - Dictionary of Materia Medica .................... 25

LETHEBY (Hy.) The Sewage Qu, Straight Line and Circle ................ 38

LIAUTARD (A.) Animal Castration $\ldots$

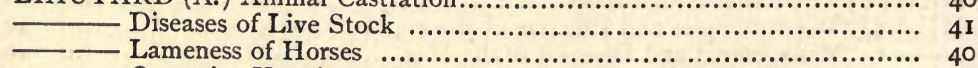

Operative Veterinary Surgery ............................................ 40

IITHGOW (R. A. Douglas) From Generation to Generation ................ 22

LOWNE (B. T.) Aids to Physiology ...................................... 37

LUNN (C.) The Philosophy of Voice ................................... 35 


\section{Baillière, Tindall, and Cox's Books.}

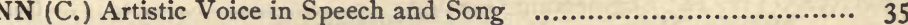

LUPTON (J. I.) Horses : Sound and Unsound............................... 40

MACDOUGALL (A. M.) The Maybrick Case ................................ 2 I

MACKENZIE (Sir M.) Diseases of the Throat (in Gant's Surgery) ........... 33

MCCAW (John) Aids to the Diagnosis and Treatment of Diseases of Children 15

MADDEN (T. More) Clinical Gynæcology .................................. 22

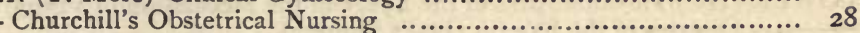

MADDICK (Distin) Stricture of the Urethra ............................... 33

MAGNE (Dr.) How to Preserve the Sight..................................... 20

MARTIN (B. R.) Diphtheria ............................................. 18

MARTIN (J. W. \& J.) Ambulance Work (Questions and Answers) ........... 8

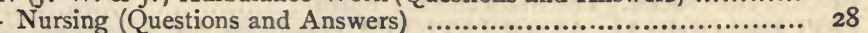

MASSE (J. N.) Text-book of Naked-Eye Anatomy ........................... 9

MAYBURY - Student's Chemistry ............................................ I5

MCARDLE (J. S.) Notes on Materia Medica. ..................................... 26

McBRIDE Anatomical Outlines of the Horse ................................ 4 I

MCLACHLAN (John) Anatomy of Surgery ................................. 33

MEARS (W. P.) Schematic Anatomy $. . . \ldots \ldots \ldots \ldots \ldots \ldots \ldots \ldots \ldots \ldots \ldots \ldots \ldots . . . \ldots \ldots$

MELDON (Austin) A Treatise on Gout ......................................... 22

MEYRICK (J. J.) Stable Management in India.................................... 4 I

MILLARD (H. B.) Bright's Disease of the Kidneys.......................... 25

MILLER (B. E.) Diseases of Live Stock ....................................... 4 I

MOLONY (M. J.) Rupture of the Perineum ..................................... $\quad 32$

MONIN (E.) Hygiene of Beauty ................................................ 24

MOORE (E. H.) Clinical Chart for Hospital and Private Practice.............. 34

MOORE (J. W.) Text Book of Eruptive and Continued Fevers................. 2 I

MORDHORST (Carl) Rheumatism. Its Treatment by Electric Massage ... 32

MORGAN (John) The Dangers of Chloroform and Safety of Ether ............ 8

MORRIS (Malcolm) The Skin (in Gant's Surgery) .......................... 33

MUCKLEY (W. J.) Student's Manual of Artistic Anatomy..................... I I

A Handbook for Painters and Art Students on the Use of Colours 16

MURRAY (R. Milne) Pregnancy............................................ Io

MURRELL (W.) Aids to Forensic Medicine and Toxicology ................... 2I Prevention of Consumption .............................................. 16

MUTER (J.) Manual of Analytical Chemistry ................................... I5

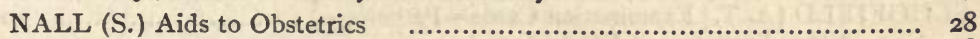

NAPHEYS (G. H.) Handbook of Popular Medicine ............................. I8

- Modern Therapeutics ................................................. 34

NATIONAL SOCIETY FOR PREVENTION OF BLINDNESS ...... 20

NEUMANN (L. G.) Treatise on Parasites and Parasitic Diseases of Domesticated Animals

NORTON (A. T.) Text-book of Operative Surgery ............................... Osteology for Students .............................................

Affections of the Throat and Larynx .......................................

Movable Atlas of the Skeleton.........................................

Clinical Lectures on Recent Surgery ...................................

OGSTON On Unrecognised Lesions of the Labyrinth ..........................

ORMSBY (L. H.) Deformities of the Human Body ............................... Phimosis and Paraphimosis ........................................

PALFREY (J.) Atlas of the Female Organs of Generation .................... I 8

PALMER (J. F.) How to Bring up Children by Hand ........................ 28

PARKE (Surgeon) Climate of Africa (in Cullimore's Book of Climates) ..... 16

PEDDIE (W.) Manual of Physics............................................. 30

PENNING (W. H.) Text-book of Field Geology ............................ 21 
PENNING (W, H.) Engineering Geology

PENNING (W. H.) Engineering Geology …........................... 2 I

PETTENKOFER (Von) Cholera : How to Prevent and Resist It ............ I

PIERSOL (G. A.) Text-book of Normal Histology ............................. 23

POLITZER (Prof.) Dissections of the Human Ear .......................... 19

Text-Book of Diseases of the Ear ................................. I9

POWER (Hy.) Movable Atlas of the Eye, and the Mechanism of Vision ...... Io

Diseases of the Eye (in Gant's Surgery) ........................... 33

POWER (D'Arcy) Handbook for the Physiological Laboratory ................. 23

POYSER (R.) Stable Management of Troop Horses in India .................. $4 \mathbf{I}$

PRATT (W.) A Physician's Sermon to Young Men ........................... 27

PROCTOR (Richd.) The Stars and the Earth .............................. I2

PSYCHOLOGIGAL ASSOCIATION'S Handbook for Attendants on the

Insane...

PURVES (L.) Aural Diseases (in Gant's Surgery) ....................................

RABAGLIATI (A.) Muscular Affections which Simulate Diseases of the

Pelvic Organs in Women

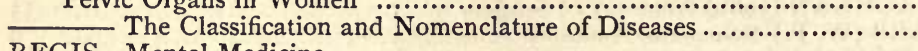

REGIS-Mental Medicine ... . ..................................................

REMSEN (Ira) Principles of Theoretical Chemistry .........................

RENTOUL-Reform of Medical Charities ....................................

REYNOLDS (R. S.) The Breeding and Management of Draught Horses.......

RICHARDS (J. M.) A Chronology of Medicine ..............................

RICHARDSON (B. W.) The Healthy Manufacture of Bread ..................

RIVINGTON (W.) Medical Education and Organization ......................

ROBERTSON (William) A Handbook of the Practice of Equine Medicine...

ROCHE (J.) Hernia and Intestinal Obstruction ....................................

ROCHET (Chas.) The Prototype of Man, for Artists ............................

ROSE (W.) Neuralgia.........................................................

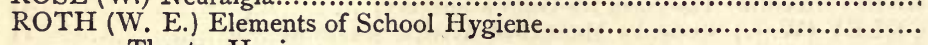
Theatre Hygiene

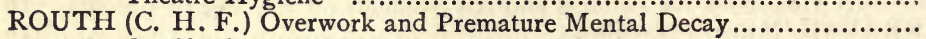
On Checks to Population

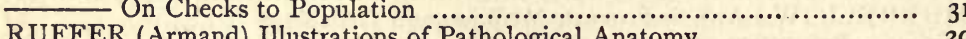

SARCEY (F.) Mind your Eyes................................................. 20

SCHOFIELD (A. T.) Examination Cards - Pathology ........................ 20

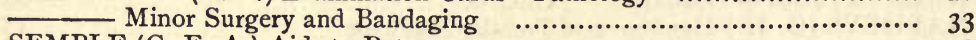

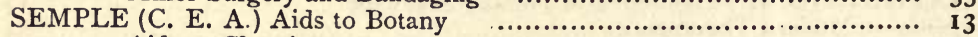

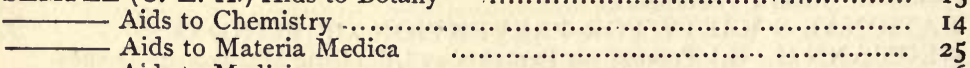

- Aids to Medicine $\quad$............................................................. 26

Aids to Pharmacy $\quad$................................................. 30

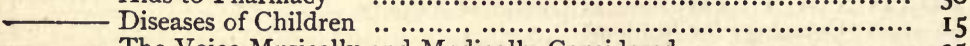

The Pocket Pharmacopœia

SEWILL (Hy.) Manual of Dental Surgery ...................................... I7

Dental Caries and the Prevention of Dental Caries .................... I

SHARMAN (J. S.) Notes on Inorganic Materia Medica ........................ 26

SIMON (W.) A Manual of Chemistry ........................................ 15

SMITH (F. A. A.) Keep your Mouth Shut ................................. 32

SMITH (F.) Manual of Veterinary Hygiene .................................. 4 I

Manual of Veterinary Physiology ............................. 4I

SOHN (C. E.) Dictionary of the Active Principles of Plants ................... 15

SPARKES (John C. L.) Artistic Anatomy …............................. II

SQUIRE (P. W.) Posological Tables ...................................... $3 \mathbf{I}$ 


\section{Baillière, Tindall, and Cox's Books.}

STARK (A. Campbell) Practical Pharmacy

STARR (M. Allen) Brain Surgery

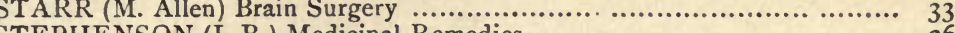

STEVENS (Geo. T.) Nervous Diseases ....................................

STEIVART (W. R. H.) Practitioner's Handbook of Diseases of the Ear...... I9

Aids to Otology ................................................ I8

STONE (G.) Translation of Politzer's Dissections of the Human Ear ......... I 9

STRAHAN (J.) Extra-Uterine Pregnancy . ................................... 28

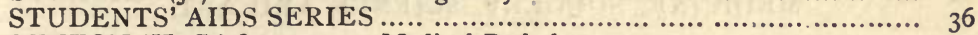

SUTTON (H. G.) Lectures on Medical Pathology ............................. 29

SUTTON (Bland) Dermoids................................................... I 7

SWEETING (R. D. R.) The Sanitation of Public Institutiuns ................ 24

SYMINGTON (J.) Anatomy of the Child ..................................... 9

TELLOR (L. V.) Diseases of Live Stock ................................... 4 I

TEULON (G.) The Functions of Vision........................................... 20

THIN (George) Introduction to Practical Histology ............................... 23

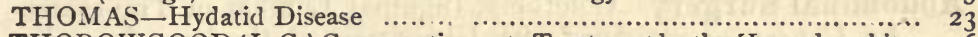

THOROWGOOD (J. C.) Consumption; its Treatment by the Hypophosphites 16 The Treatment of Bronchial Asthma ............................... I2 Aids to Physical Diagnosis .......................................... I 8

THUDICIHUM (J. L. W.) The Physiological Chemistry of the Brain ......... 13 Aids to Physiological Chemistry ................................... $3^{8}$ Aids to Public Health.................................................. $3 \mathbf{I}$

Polypus in the Nose ............................................. 31

The Coca of Peru, and its Remedial Principles........................ If

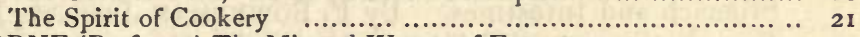

TICHBORNE (Professor) The Mineral Waters of Europe ...................... 27

TIDY (Meymott) and CIARKE (Percy) Medical Laws ........................ 26

TIMMS (G.) Consumption ; its Nature and Treatment ........................ 16

Alcohol in some Clinical Aspects, a Remedy, a Poison ............... 8

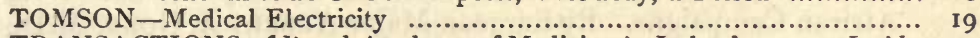

TRANSACTIONS of Royal Academy of Medicine in Ireland ......... Inside cover

TUCKEY (C. Lloyd) Psycho-Therapeutics …................................ 24

TURNER (Dawson) Manual of Medical Electricity ............................. 19

TYSON (J.) The Urine, a Guide to its Practical Examination ............... 35

UNDERWOOD (Arthur S.) Aids to Dental Surgery ........................ I7

USHER Aids to Dental Histology …...................................... I7

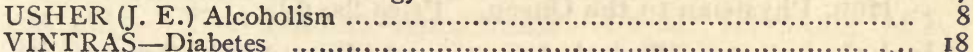

WAGSTAFFE (W. W.) Atlas of Cutaneous Nerve Supply ..................... 27

WALDO and WALSH-Bread, Bakehouses................................. I 2

WALLACE (J.) Localised Peritonitis........................................... 29

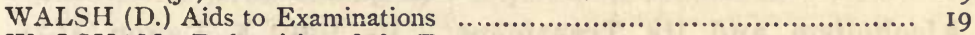

WALSHAM-Deformities of the Foot ................................. 2 I

WALSHAM and POWER-Surgical Pathology .............................. 33

WELPLY (J. J.) Creameries and Infectious Diseases .......................... 24

WHERRY (Geo.) Clinical Notes on Nerve Disorders .......................... 27

WILLIAMS (J. W.) Aids to Biology ... ................................ I3

WILLIAMSON (J. M.) Ventnor and the Undercliff........................... 16

WILLSON (A. Rivers) Chemical Notes for Pharmaceutical Students ......... I 5

WILSON (J.) A Manual of Naval Hygiene .................................. 24

WINDLE (B. C. A.) Proportions of the Human Body ........................ 12

WINSLOW (L. S. Forbes) Fasting and Feeding .......................... 20

WITKOWSKI (G. J.) Movable Atlases of the Human Body .................. Io

YONGE (E. S.) Aids to Surgical Anatomy ............................. 36

YOUNG (J. K.) Orthopedic Surgery ........................................ 2 I 
 \\ Baillière, Tindall, and Cox's Books.}

AN

\section{ALPHABETICAL INDEX OF WORKS,}

IN

MEDICINE, SURGERY, SCIENCE AND ART,

PUBLISHED BY

\section{BAILLIERE, TINDALL, \& COX,}

Abdominal Surgery. Colotomy, Inguinal, Lumhar or Transverse; for Cancer, or Stricture with Ulceration, of the large Intestine. By Herbert W. Allingham, F.R.C.S., Surgeon to the Great Northern Hospital, Assistant Surgeon to St. Mark's Hospital for Diseases of the Rectum, Surgical Registrar to St. George's Hospital. With six plates and numerous illustrations. Price 6s.

Abdominal Surgery. The Surgical Diseases and Injuries of the Stomach and Intestines. By F. Bowreman JessetT, F.R.C.S. Eng., Surgeon to the Cancer Hospital. Copiously illustrated. Price $7 \mathrm{~s}$. 6d.

Africa. A Contribution to the Medical History of our West A frican Campaigns. By Surgeon-Major Albert A. Gore, M.D., Sanitary Officer on the Staff. Price 10s. $6 \mathrm{~d}$.

Africa. Life on the Gold Coast. A Description of the Inhabitants, their Modes and Habits of Life ; Hints to Travellers and others in Western Africa. By Surgeon-General GoRDon, M.D., C.B., Hon. Physician to the Queen. Price 2s. 6d.

Alcohol, in some Clinical Aspects: A Remedy, a Poison. By Godwin Trmms, M.D., M.R.C.P. Lond., Senior Physician to the North Londun Consumption Hospital. Price 1s.

Alcoholism and its Treatment. By JoHN E. Usher, M.D., F.R.G.S. Price 3s. 6d.

"Will be found interesting and suggestive."-The Times.

"A very full account of the methods of treating the disease of inebriety is contained in this interesting work."-British Medical Journal.

Ambulance Work. Questions and Answers on "First Aid to the Injured." By JoHN W. Martin, M.D., and JohN MarTiN, F.R.C.S. Twentieth thousand. Price 1s. net.

Anæsthetics. The Dangers of Chloroform and the Safety and Efficiency of Ether in Surgical Operations. By JoHn Morgan, M.D., F.R.C.S. Second thousand, price 2 s'. 


\section{Baillière, Tindall, and Cox's Books.}

Anæsthetics. Selected Methods in the Administration of Nitrous Oxide and Ether. By Frederic HewitT, M.A., M.D. Cantab., Lecturer on Anæsthetics at the London Hospital. Price 2s. 6d.

Anæsthetics : Ancient and Modern. Their Physiological Action, Therapeutic Use, and Mode of Action. By GEORGE For, F.R.C.S., Surgeon to the Richmond Hospital. Price 3s. 6d. net.

Anatomography; or, Graphic Anatomy. A new method of grasping and committing to memory the most difficult points required of the student. By W. DARLING, M.D., F.R.C.S. Eng., Professor of Anatomy in the University of New York. Price 1s.

Anatomy. Aids to Anatomy. By George Brown, M.R.C.S., and P. Macleod Yearsley, F.R.C.S. Price 23.6d. cloth, 2s. sewn.

Anatomy. Text-Book of Naked-Eye Anatomy. With 113 Steel Plates, designed under the direction of Professor MAsse. Text by JaS. CANTLIE, M.B., C.M. (Honours), F.R.C.S., Charing Cross Hospital. Third edition. Plain, 25s., coloured, 50s., half calf.

Anatomy. The Fssentials of Anatomy. A Text-book for Students and a book of easy reference to the Practitioner. By W. Darling, M.D., F.R.C.S., and A. L. RANNeY, M.D. 12s. 6d.

Anatomy. The Pocket Gray, or Anatomist's Vade-Mecum. Com. piled from the works of Gray, Ellis, Holden, and Leonard. By E. CotTerell, F.R.C.S. Eng., late Demonstrator of Anatomy, University College, London. Fourth edition, 3s. 6d.

"A marvellous amount of information condensed into a remarkably small space."-Med. Press.

Anatomy. The Pocket Anatomist. By H. Leonard, M.D. Eularged Edition, illustrated. Price 3s. 6d.

Anatomy. Schematic Anatomy; or Diagrams, Tables and Notes treating of the Association and Systematic arrangement of Structural Details of Human Anatomy. By WiLliam P. Mears, M.B., Professor and Examiner in Anatomy at the University of Durham. Profusely illustrated. Price 7s. 6d.

Anatomy. Anatomy of the Child. With 14 coloured plates and 33 woodcuts. By Johnsun Srmington, M.D., F.R.S.E., F.R.C.S.E., Lecturer on Anatomy, Edinburgh. Price 42s.

Anatomy of the Inguinal and Femoral Regions in Relation to Hernia. By E. LEDWICH, Lecturer on Anatomy in the Ledwich School of Medicine, Dublin. Price 3s. 


\section{Baillière, Tindall, and Cox's Books.}

Anatomy. Human Anatomy and Physioloy, illustrated by a series of Mcvable Atlases of the Human Body, showing the relative positions of the several parts, by means of Superposed Coloured Plates, from the designs of Professor G. 'J. WITKowski, M.D. Each part complete in itself. Price 7s. 6 d. net.

Part I.-Neck and Trunk. With Text Descriptive and Explanatory of the physiology and functions of the several parts. By Robert Hunter Semple, M.D., F.R.C.P. Lond. Price 7s. 6d.

The same enlarged to Life Size. Price £2 2s.

Part II.-Throat and Tongue, showing the Mechanism of Voice, Speech, and Taste. Text by Linnox Browne, F.R.C.S. Ed. Price 7s. 6d.

Part III.-The Female Organs of Generation and Reproduction. Text by James Palfrey, M.D., M.R.C.P. Lond., late Senior Obstetric Physician, London Hospital. Price 7s. 6d.

Part IV.-The Eye and the Apparatus of Vision. Text by Henry Power, F.R.C.S., Senior Ophthalmic Surgeon to St. Bartholomew's Hospital. Price 7s. 6d.

Part V.-The Ear and Teeth. The Mechanism of Hearing, and of Mastication. Text of the Ear by Lennox Browne, F.R.C.S.E. The Teeth by H. SEwILl, M.R.C.S. Price 7s. 6d.

Part VI.-The Brain and Skull. (Cerebrum, Cerebellum, and Medulla Oblongata.) Text by T. STRETCH Dowse, M.D., F.R.C.P. Ed. Price 7s. 6d.

Part VII.-The Male Organs of Generation. Text by D. Campbell Black, M.D., Physician to the Glasgow Royal Infirmary. Price 7s. 6d.

Part VIII.-The Skeleton and its Articulations, showing the Bones and Ligaments of the Human Body and Limbs. Text by A. T. Norton, F.R.C.S. Price 7s. 6 d.

Part IX.-The Hand; its Bones, Muscles and Attachments. Text by Jas. Cantuie, M.B., F.R.C.S. Price 7s. 6 d.

Part X.-The Foot; its Bones, Muscles and Attachments. Text by Stanley Boyd, M.B., B.S. Lond., F.R.C.S., Assistant Surgeon, Charing Cross Hospital. Price 7s. 6d.

Part XI.-Progress of Gestation. A Synopsis of Practical Obstetrics. Text by R. Milne Murray, F.R.C.P. Edin., M.B. Edin.' Price 7s. 6d.

The Set of Eleven Parts, complete in cloth-covered Box, with lock and key, £4 net.

** No such simple, reliable, and comprehensive method of learning the several parts, positions, and functions of the body has hitberto been attempted; the entire Series being unique, will be most valuable to the Teacher, the Student, and to all who wish to become acquainted with the anatomy and physiology of the human economy. 
Apoplexy. On Stertor, Apoplexy, and the Management of the Apoplectic State. By RoBerT L. Bowles, M.D., F.R.C.P. Lond., Consulting Physician to the Victoria Hospital, and to the St. Andrew's Convalescent Hospital, Folkestone. 'With 13 Illustrations. Price $4 \mathrm{~s}$. $6 \mathrm{~d}$.

"Based on extensive clinical and experimental investigation. The principles deserve to be more widely known and acted on."-British Medical Journal.

"A book which is at present the only authority on the subject."-Medical Press.

Apoplexy. Diagnosis and Treatment of Apoplexy. By T. STRETCH Dowse, M.D., F.R.C.P.E., formerly Medical Superintendent, Central London Sick Asylum. Price 1s.

Army Hygiene. Lessons in Military Hygiene and Surgery. By Surgeon-General Gordon, M.D., C.B., Hon. Physician to H.M. the Queen. Illustrated. Price 10s. 6d.

Artistic Anatomy. Anatomy of the External Forms of Man, for the use of Artists, Sculptors, etc. By Dr. J. FAU. Used at the Government School of Art, South Kensington. Twenty-nine plates. Folio. New edition. 30s. coloured, 15s. plain.

Artistic Anatomy. Elementary Anatomical Studies of the Bones and Muscles, for Students and Schools, from the drawings of J. Flaxman, R.A. Lately used as a Text-book in the Art Schools at South Kensington. 20 plates, with Text, price 2s.

Artistic Anatomy. The Student's Manual of Artistic Anatomy. With 25 etched plates of the bones and surface muscles of the human figure. By W. J. MUCKLEY. Used at the Government School, South Kensington. Second edition. Price 5s. 6d.

Artistic Anatomy. Elementary Artistic Anatomy of the Human Body. From the French of Dr. FAU. With English Text. Used at the Government School of Art, South Kensington. Price 5s.

Artistic Anatomy. Description of the Bones and Muscles that influence the External Form of Man. With 43 plates. By JoHN C. L. SPARKeS, Principal of the National Art Training School, South Kensington. Adopted as a text-book at the Government Art Schools. Price 7s. 6d.

Artistic Drawing. Elementary (Second Grade) Perspective (Theory and Practice), containing 30 block illustrations, 21 plates, and many examination exercises. Used at the Government Science and Art Schools. By H. J. Dennis, Art Master, Lambeth School of Art, Dulwich College, etc. Price 2s. 6d.

Artistic Drawing. Advanced (Third Grade) Perspective, for the use of Art Students. By H. J. Denvis. Used at the Science and Art Schools. In two parts, 7s. 6d. each. Part 1, Angular and Oblique Perspective. Part 2, Shadows and Reflections ; or, halfbound leather in one vol., price 15s. 
Artistic Drawing. The Prototype of Man, giving the natural laws of Human proportion in both sexes. A manual for artists and professors of drawing. By Chas. Rochet, of Paris. Price 1s.

Artistic Drawing. A Manual of the Proportions of the Human Body for Artists. By Bertram C. A. Windle, M.A., M.D., D.Sc., Queen's Professor of Anatomy in the Mason College, Professor of Anatomy to the Royal College of Artists, and Lecturer in the Municipal School of Birmingham. Price 2s.

Artists' Colours. Their Preparation, Uses, etc. (See Colours.)

Asthma. On Asthma and Chronic Bronchitis - their Causes, Pathology and Treatment. Lettsomian Lectures. By J. C. Thorowgood, M.D., F.R.C.P. London, Senior Physician to the City of London Hospital for Diseases of the Chest. Fourth edition. Price 4s.

Astronomy. The Stars and the Earth; or, Thoughts on Time, Space, and Eternity. With Notes by R. A. Proctor, B.A. Fourteenth thousand. Price 1s.

Ataxia. Nervous Affections associated with the Initial or Curative Stage of Locomotor Ataxy.: By T. Stretch Dowse, M.D., F.R.C.P.E. Second edition. Price 2s.

Bacteria. Bread, Bakehouses and Bacteria. By F. J. WALDO, M.D. Cantab., and David WaLSh, C.M. Edin. Price 2s.

Bacteriology. Researches in Micro-Organisms, including recent Experimentsin the Destruction of Microbes in Infectious Diseases, etc. By A. B. GRIFFIths, Ph.D., F.C.S., F.R.S.E. With 52 Illustrations. Price 6s.

"An enormous amount of material, the author has taken great trouble to collect a large number of the references bearing on the points he mentions."-Lancet.

"The work...may be recommended to those who wish to have in a convenient form a very large number of facts and references relating to bacteria."-British Medical Journal.

Bacteriology. A Parasitic or Germ Theory of Disease: the Skin, Eye, and other affections. By JABEZ HoGG, M.R.C.S. Second edition. Price 2s. 6 d.

Bacteriology. Guide to the Demonstration of Bacteria in the Tissues. By Dr. H. KüHNE, of Wiesbaden. Translated by VINCENT Dormer Harris, M.D. Lond., F.R.C.P. Price 2s. $6 \mathrm{~d}$.

Bacteriology. Microbes in Fermentation, Putrefaction, and Disease. By Charles Cameron, M.D., LL.D., M.P. Price 1s.

Professor Tyndall, F.R.S., writes: "Matthew Arnold himself could not find fault with its lucidity, while as regards knowledge and grasp of the subject I have rarely met its equal." 


\section{Baillière, Tindall, and Cox's Books.}

Bandaging. A Manual for Self-instruction. By C. H. Leonard, A.M. M.D., Professor of Diseases of Women in the State College, Michigan. With 139 illustrations. Price 3s. 6d.

Biology. Aids to Biology. Prepared to meet the requirements of students reading for the first examination of the Conjoint Board. By Joseph W. Williams. Price 2s. sewn, 2s. 6d. cloth.

Bladder. On Diseases of the Bladder, Prostate Gland, and Urethra. By F. J. Gant, F.R.C.S., Senior Surgeon to the Royal Free Hospital. Fifth edition. Price 12s. 6d.

Botany. A Dictionary of British Plants and Flowers; their names, pronunciation, origin, etc. By H. P. Fitzgerald. Price 2s. 6d.

Botany. Aids to Botany. Outlines of the Elementary Facts, including a Description of some of the most important Natural Orders. By C. E. Armand Semple, B.A., M.B. Cantab., M.R.C.P. Lond. Price 2s. 6d. cloth; 2s. paper wrapper.

Botany. The Student's Botany. Encyclopædic Glossary. By E. MacDowel Cosgrave, M.D., Lecturer on Botany, Carmichael College. Price 2s. 6d.

Brain. The Building of a Brain. By E. H. ClaRKE, M.D. (author of "Sex in Education"). Price 5s.

"Carefully and elegantly written, and full of sound physiology."-Laneet:

Brain. On Irritable Brain in Children. By W. H. DAY, M.D., M.R.C.P. Lond., Physician to the Samaritan Hospital for Women and Children. Price $1 \mathrm{~s} .6 \mathrm{~d}$

Brain. The Physiological and Chemical Constitution of the Brain, based throughout on original researches. By J. L. W. THUDI CHUM, M.D., F.R.C.P. Lond. Price 10s. 6d.

Brain. Syphilis of the Brain and Spinal Cord, showing the part which this agent plays in the production of Paralysis, Epilepsy, Insanity, Headache, Neuralgia, Hysteria, and other Mental and Nervous Derangements. By T. Stretch Dowse, M.D., F.R.C.P. Ed. Second edition, illustrated. Price 5s.

Brain. On Brain and Nerve Exhaustion (Neurasthenia), and on the Exhaustions of Influenza. By the same Author. Price 2s. 6d.

Brain. Microscopical Examination of the Human Brain, Methods of Research, etc. By Edwin Goodall, M.D. Lond. Price 5s.

Bronchitis. Chronic Bronchitis : its Forms and Treatment. By J. Milner Fothergill, M.D. Ed., M.R.C.P. Lond. Price 4s. 6 d.

"It bristles with valuable hints for treatment."-British Medical Journal.

"The pages teem with suggestions of value."-Philadelphia Medical Times.

Burmah. Our Trip to Burmah, with Notes on the Ethnology, Geography, Botany, Habits and Customs of that Country, by Surgeon-General GorDon, C.B., M.D., Physician to the Queen. Illustrated with numerous Photographs, Maps, Coloured Plates, and Sketches in gold by native Artists. Price 21s.

"We lay down this book, impressed with its many beauties, its amusing sketches and anecdotes, and its useful and instructive information."-The Times. 
Cancer and its Complications. Its Local Origin, Preventive and Curative Treatment. By C. E. Jennings, F.R.C.S. Eng., M.S., M.B. Second edition. Price 3s. 6d. net.

Cancer. Lectures on Cancer of the Uterus, with Cases. By F. B. Jessett. Price 3s. 6 d.

Cancer of the Mouth, Tongue and Esophagus. By F. BowrEMAN JESSETT, F.R.C.S. Eng., Surgeon to the Cancer Hospital. 6s.

Cancer, Sarcoma, and other Morbid Growths considered in relation to the Sporozoa. By J. JACKson Clarke, M.B. Lond., F.R.C.S., Curator of the Museum, and Pathologist at St. Mary's Hospital. Illustrated. Price 3s. 6 d. net.

Case Books. A Pocket Case-book for Practitioners and Students. With diagrams, charts, and suggestions for note-taking. By Alex. Theodore Brand, M.D., C.M. Bound in limp leather cover. Price 4s. Loose sheets per doz. 1s., 50 3s. 6d., $1006 \mathrm{s.}$

Case Taking. Cardiac Outlines for Clinical Clerks and Practitioners; and First Principles in the Physical Examination of the Heart. By W. EwarT, M.D., F.R.C.P. Lond., Physician to St. George's Hospital, London. With fifty illustrations. Price 5s. 6d.

* * A supply of thoracic and cardiac outlines ( $4 \frac{1}{2}$ by $3 \frac{3}{4}$ inches), on gummed paper, is included in each copy. Separately $9 \mathrm{~d}$.

Case Taking. Symptoms and Physical Signs, a formulary for medical note-taking, with examples. By the sameAuthor. Price 2s.

Case Books. Student's Case-book. For recording cases as seen, with full instructions for methodizing clinical study. By GEORG t Brown, M.R.C.S., Gold Medallist, Charing Cross Hospital. Fourth thousand, cloth. Price 1s. net.

Case-book. Suggestions for a plan of taking notes in medical cases. By Geo. F. Duffey, M.D. Dublin. Price 6d.

Catarrhs. Home Treatment for Catarrhs and Colds. By Leonard A. Dessar, M.D. Illustrated. Price 5s.

Chemistry. Aids to Chemistry. By C. E. Armand Semple, B.A., M.B. Cantab., M.R.C.P. Lond.

Part I.-Inorganic. The Non-metallic Elements. Price 2s. 6d. cloth; 2s. paper wrapper.

Part II.-Inorganic. The Metals. Price 2s. 6d. cloth; 2s. paper. Part III.-Organic. Cloth, 2s. 6d. ; paper, 2s.

Part IV.-Tablets of Chemical Analysis. Price 1s. 6d. and 1s.

"Students preparing for Matriculation at the London University, and other Examinations, will find it simply invaluable." - Students' Journal. 


\section{Baillière, Tindall, and Cox's Books.}

Chemistry. The Student's Chemistry. Part I. The Nonmetallic Elements. By A. C. Maybury. Price 4s.

Chemistry. A Manual of Chemistry ; a complete guide to Lectures and Laboratory work for beginners in Chemistry, and a textbook for students in Medicine and Pharmacy. By W. Srmon, Ph.D., M.D. Coloured plates, 56 Chemical reactions. $15 \mathrm{~s}$.

Chemistry. Dictionary of the Active Principles of Plants: Alkaloids, Bitter Principles, Glucosides, with tabular summary and classification of Reactions. By CHARLes E. SoHn, F.I.C., F.C.S. An entirely original book. Price 10s. 6d.

Chemistry. Plant Analysis, Quantitative and Qualitative. By G. DragendorfF, Professor of Chemistry and Pharmacy in the University of Dorpat. Price 7s. 6d.

Chemistry. The Principles of Theoretical Chemistry, with special reference to the Constitution of Chemical Compounds. By IRA Remsen, M.D., Ph.D., Professor of Chemistry in the John Hopkins University. 4th edit., enlarged and revised. 7s. 6d.

Chemistry. The Student's Hand-book, with Tables and Chemical Calculations. By H. Leicester Greville, F.I.C., F.C.S. Second edition. Price 6s.

Chemistry. Chemical Notes for Pharmaceutical Students.

A. Pivers Willson. Second edition. Price 3s. 6d.

"Of exceeding value to students going up for examination."-Pharmaceutical Journal.

Chemistry. A Short Manual of Analytical Chemistry for Laboratory Use. By John Muter, Ph.D., M.A., F.C.S. New edition in preparation.

Children. The Diseases of Children : their History, Causes and Treatment. By C. E. Armand Semple, B.A., M.B. Cantab., M.R.C.P. Lond. Price 6s.

Children. Aids to the Diagnosis and Treatment of Diseases of Children. By JoHn McCAw, M.D., L.R.C.P. Price 3s. 6d. cloth ; 3s. paper cover.

Children. Confidential Chats with Mothers on the healthy rearing of Children. By Mrs. Bowdich. Price 2s.

Children. On Tetany in Young Children. By J. ABERCRombie, M.D., M.R.C.P. Lond. Price 2s.

Cholera: How to Prevent and Resist it. By Professor von PetrenkOFer and T. Whiteside Hime, A.B., M.B. Second edition. Illustrated. Price 3s. 6d.

Cholera. The Cholera Microbe and How to Meet It. Rear at the Congress of the British Medical Association. By Charles Cameron, M.D., LL.D., M.P. Price 1 s. 


\section{Baillière, Tindall, and Cox's Books.}

Climatology. Ventnor and the Undercliff. By J. M. Williamson, M.D., M.B. Ed., Hon. Surgeon to the National Hospital for Consumption. Second edition, price 1s.

Climatology. The Island of Madeira: A Resort for the Invalid and a Field for the Naturalist. By Surgeon-Genl. C. A. Gordon, M.D., C.B., Hon. Physician to the Queen. Price 2s. 6d.

Climatology. The Book of Climates in all Lands. A Handbook for Travellers, Invalids, and others in search of Health and Recreation. By D. H. CULlimore, M.D., M.R.C.P. Lond. With a chapter on the Climate of Africa by Surgeon PARKE, D.C.L. Second edition, price 4 s. 6 d.

"A very useful book."-The Graphic.

"There is much which entitles it to a large circulation."-- Westminster Revievo.

"A work of supreme interest to the traveller in search of health."-Freeman's Journal.

Coca. The Coca of Peru, its Remedial Principles, and Healing Powers. By J. L. W. Thudichum, M.D., F.R.C.P. Price 1s.

Colours. A Hand-book for Painters and Art Students, on the use of Colours, Vehicles, etc. By W. J. Muckley. Fourth edition enlarged. Price $4 \mathrm{~s}$.

Consumption. Consumption as a Contagious Disease ; the Merits of the Air of Mountains and Plains. By D. H. Cullimore, M.D., M.R.C.P. Lond., formerly H.M. Indian Army. Price 5s.

Consumption. Consumption and its Treatment by the Hypophosphites. By JонN C. Thorowgood, M.D., F.R.C.P. Lond., Physician to the City of London Hospital for Diseases of the Chest, Victoria Park. Third edition, price 2s. $6 \mathrm{~d}$.

Consumption. Lectures on the Prevention of Consumption. By Wrlutam Murrell, M.D., F.R.C.P. Lond., Physician to Outpatients at the Westminster Hospital. Price 3s. 6d.

Consumption. A Re-investigation of its Causes. By C. W. De Lacy Evans, M.R.C.S. Eng. Price 2s. 6d.

Consumption. How to Prevent and Treat Consumption. By G. Rutland Howat, B.A. Lond, Price 2s. 6d.

Consumption. An Essay on Consumption: Its True Nature and Successful Treatment. By Godwin W. Timms, M.D. Lond. Second edition, revised and enlarged, price 10s. $6 \mathrm{~d}$.

Consumption. Tuberculosis from a Sanitary and Pathological Point of View. By G. Fleming, C.B., F.R.C.V.S., President of the Royal College of Veterinary Surgeons. Price 1s. 


\section{Baillière, Tindall, and Cox's Books.}

Consumption. The Pathology of Tuberculosis (Pulmonary Tuberculosis and Tubercular Phthisis). Post Graduate Lectures delivered at Queen's Hospital, Birmingham, 1891. By GEo. F. Crooke, M.D., Physician and Pathologist to Queen's Hospital, and Lecturer on Pathology in Queen's College. Price 2s. 6d.

Deaf-mutism. On the Education of Deaf-mutes by Lip-Reading and Articulation. By Professor Hartmann. Translated by Dr. Patterson Cassells. Price 7s. 6d.

"The instruction of deaf-mutes is here rendered easy."-Athencum.

"We can honestly recommend it to anyone seeking for knowledge." - The Lancet.

Deformities. Deformities of the Human Foot, by W. J. WALSHAM, M.B., F.R.C.S., Senior Assistant Surgeon to St. Bartholomew's Hospital. Price 18s.

Deformities. The Nature and Treatment of Deformities of the Human Body. By LaMbert H. OrMSBy, M.B. Dub., Surgeon to the Meath Hospital and Dublin Infirmary. Price 5s.

Deformities. The Surgical Treatment of Deformities. By WM. ADAMS, F.R.C.S. Price 2s. 6 d.

Deformities. Three Lectures on the Growth Rates of the Body and the Limbs, in Relation to the Processes of Rectification of Deformity. By Walter Pye, F.R.C.S. Eng. Price 1s.

Dental Surgery. A Manual of Dental Surgery : Including Special Anatomy and Pathology. For Students and Practitioners. By HeNRy SewILL, M.R.C.S., L.D.S. Eng. Third edition, with upwards of 200 illustrations, chiefly original. Price 10s. 6d. Dental. Aids to Dental Surgery. By ARTHUR S. UNDERWOod, M.R.C.S., L.D.S. Eng. Price 2s. 6d. cloth; 2s. paper.

Dental. Aids to Dental Histology. By the same Author. Price 2s. 6d. cloth; 2s. paper wrapper.

Dental. Manual for the Dental Laboratory. A Practical Guide to its Management, Economy, and Methods of Manipulation. By Charles Hunter, Author of "A Treatise on Mechanical Dentistry." Price 3s. 6d.

Dermatology. (See Skin.)

Dermoids. A Course of Lectures delivered at the Royal College of Surgeons, 1889, on "Evolution in Pathology." By J. BLAND SuTToN, F.R.C.S., Hunterian Professor. Illustrated. Price 3s.

"We commend the study of this book to all interested in the elucidation of pathological problems: - -Then Lancest.

Diabetes. Diabetes and its Treatment. By A. Vintras, M.D., Senior Physician to the French Hospital, London. 1s 6d.

Diagnosis. The Pl.vsiological Factor in Diagnosis. By J. MILNER Fothergill, M.D., M.R.C.P. Lond. Second ed. Price 7s. 6d.

"An exceedingly clever and well-written book, put together in a very plain, practical, and taking way."-Edinburgh Medical Journal. 
Diagnosis, Aids to. Three Parts. Price 1s. and 1s. 6d. each. Part I. - Semeiological. By J. Milner Fothergill, M.D. Part II.-Physical. By J. C. Thorowgood, M.D., F.R.C.P. Part III.-What to Ask the Patient. By J. Mrlner Fothergill, M.D.

Or in 1 vol. Edited by Dr. Thorowgood. Price 3s. 6d. cloth. "A mine of valuable information."-Edinburgh Medical Journal.

Dictionary. Illustrated Dictionary of Medicine, Biology, and Allied Sciences, including Pronunciation, Derivation, etc. By George M. Gould, A.M., M.D. Half morocco. Price 40s. net.

Diphtheria. A Practical Treatise on Diphtheria and its successful Treatment. By B. R. Martin, M.B. Dub. Second Edition. Price 2s.

Diphtheria. Diphtheria and its Associates. By Lennox Browne, F.R.C.S. Edin. 18s.

Diseases. The Classification and Nomenclature of Diseases. By A. Rabagliati, M.A., M.D., Senior Surgeon Bradford Infirmary, Surgeon to the Children's Hospital. Price 2s. 6d.

Domestic Medicine. Handbook of Popular Medicine for family instruction, colonists and others out of reach of medical aid. By G. H. NAPHeYs, A.M., M.D. With movable plate and 100 illustrations. Price 7s. 6 d.

Diet. How to Prolong Life. Showing the Diet and Agents best adapted for a lengthened prolongation of existence. By C. W. De Lacy Evans, M.R.C.S. Second edition. Price 5s.

(See also Food.)

Ear. Diseases of the Ear. By GEonge P. FIELD, M.R.C.S., Aural Surgeon to St. Mary's Hospital, and Lecturer on Aural Surgery. Fifth edition, enlarged, with 23 coloured plates and numerous woodcuts. Price 12s. 6d.

Ear. The Pathology and Treatment of Suppurative Diseases of the Ear. By the same author. Price 2s. 6d.

Ear. On Unrecognised Lesions of the Labyrinth. The Cavendish Lecture, 1890. By Alex Ogston, M.D., C.M., Regius Professor of Surgery, University of Aberdeen. Illustrated. Price 1s.

Ear. On Vascular Deafness. By RoBert J. Cooper, M.D., Trinity College, Dublin. Price 3s. 6d.

Ear. Aids to Otology. By W. R. H. Stewart, F.R.C.S.E. Price 2s. 6d. cloth.

Ear. Subjective Noises in the Head and Ears. Their Etiology, Diagnosis and Treatment. By H. Macnaughton Jones, M.D., F.R.O.S. I. and E. Profusely illustrated, price 4s. 6d. 


\section{Baillière, Tindall, and Cox's Books.}

Ear. The Hygiene of the Ear. By Cavaliere Vincenzo CozzoLINo, Professor in the Royal University of Naples, and Director of the Hospital Clinic for Diseases of the Ear, Nose, and Throat. Translated from the fifth Italian edition by James Erskine, M.A., M.B. Price 1s.

Ear. Practitioner's Hand Book of Diseases of the Ear and NasoPharynx. By Dr. H. Macnaughton Jones and Mr. W.R. H. STEWART, F.R.C.S.E. Fifth edition, with plates and numerous woodcuts. Price 10s. 6 d.

Ear. Text-book of Diseases of the Ear and Adjacent Organs. By Professor PoLITzer, of Vienna. Translated from the third German edition by OSCAR DoDD, M.D., and Edited by SIr WM. Dalby, B.A., M.B., F.R.C.S. Profusely illustrated. Price 21s.

Ear. The Anatomical and Histological Dissection of the Human Ear, in its Normal and Diseased Conditions. By Professor Politzer, of Vienna. Translated at the author's request by George Stone, F.R.C.P. Ed. Profusely illustrated. 10s. 6d.

Electricity. A Manual of Practical Medical Electricity. By DAwson TURNer, B.A., M.D., F.R.C.P. Ed., M.R.C.P. Lond. Profusely Il ustrated. Price 7s. 6d.

"Valuable alike to students and practitioners."-Practitioner.

"We cordially recommend this text-book, both to the student and the practitioner, as a thoroughly reliable and practical manual of the subject which it professes to teach." -Medical Press and Circular.

"Dr. Turner gives us an admirable and complete exposition of electricity, as applied to the practice of medicine and surgery, which is well up to date, and conveyed in a clear and lucid manner, such as will readily attract the attention of practitioners and students, for whom the book is intended."-Medical Reprints.

Electricity. Electricity in General Practice. By W. BoLtoN Touson, M.D. Price 2s. 6d.

Etiquette. A few Rules of Medical Etiquette. By a L.R.C.P. Lond. Price 1s.

Examinations. Aids to Examinations. By D. WALSH, M.B., C.M, L.R.C.P. Being Questions and Answers on Materia Medica, Medicine, Midwifery, Pathology, and Forensic Medicine. New edition. Price 2s. 6d. cloth; 2s. paper wrapper.

Examinations. A Guide to the Examinations of the conjoint Board in England and for the Fellowship of the College of Surgeons, with Examination Papers. By F. J. GANT, F.R.C.S. Sixth edition, revised and enlarged. Price 5s. net.

Examinations. A Guide to the Examinations of the Apothecaries' Society of London with Questions, Tables on Materia Medica, etc. By W. E. Dawson, L.S.A. Second edition. Price 2s. 6d.

" May be studied with great advantage by a student, shortly before presenting himself for examination."-British Medical Journal. 
Examinations. Examination Questions on the Medical Sciences, including the Army, Navy and University Examinations. By James Greig Leask, M.B. Abdn. Price 2s. 6 d.

Examinations. Practical Pharmacy for Medical Students; specially adapted for the Examination in Practical Pharmacy of the Conjoint Board. By A. CAMPBell Stark, Demonstrator on Materia Medica and Pharmacy at St. George's Hospital. Price 3s. $6 \mathrm{~d}$., or interleaved for note-taking, $4 \mathrm{~s}$. $6 \mathrm{~d}$.

Examination Cards : Questions and Answers. By A. T. ScHoFIELD, M.D. Pathology, 2 sets, 9d. each, net. Minor Surgery, Bandaging, etc., 9d., net.

Eye. Haab's Atlas of Ophthalmoscopy and Ophthalmic Diagnosis. Translated from the German and edited by ERNEST CLARKE, M.D. Lond., F.R.C.S.

[n the press.

Eye. Aids to Ophthalmic Medicine and Surgery. By J. Hutchinson, jun., F.R.C.S., Ophthalmic Surgeon to the Great Northern Hospital. Cloth, 2s. 6d.

Eye. Ophthalmic Notes. A Pocket Guide to the Nature and Treatment of Common Affections of the Eye. By A. Vernon Ford, M.R.C.S. Eng., L.K.Q.C.P. Ire. Price 2s. 6d.

Eye. The Detection of Colour Blindness, from a practical point of view. By F. W. EdRIDGe-Green, M.D., F.G.S. Price 1s.

Eye. The Cure of Cataract and other Eye Affections. By JABEZ HoGg, M.R.C.S., Consulting Surgeon to the Royal Westminster Ophthalmic Hospital. Third edition. Price 2s. 6d.

Eye. On Impairment or Loss of Vision from Spinal Concussion or Shock. By the same Author. Price 1s. 6d.

Eye. The Functions of Vision and its Anomalies. By Dr. Giraud TEulon. Translated by LloYd Owen, F.R.C.S.I., Surgeon to the Midland Eye Hospital, Ophthalmic Surgeon to the Hospital for Sick Children, Birmingham. Price 5s.

Eye. Movable Atlas of the Eye and the Mechanism of Vision. By Prof. G. J. Witkowski. Price 7s. 6d. (See Anatomy.)

(The following four uorks have been translated for the National Society for the Prevention and Cure of Blindness.)

The Causes and Prevention of Blindness. By Professor Fuchs, University of Liége. Price 7s. 6d.

Mind your Eyes. By F. SARceY. Price 2s. 6 d.

How to Preserve the Sight. By Dr. Magné. Price 6 d.

On Spectacles, their History and Uses. By Prof. Horner. Price 6d.

Fasting and Feeding, Psychologically considered. By L. S. Forbes Winslow, M.B. Cantab., D.C.L. Oxon. Price 2s. 
Fever. On the Endemic Hæmaturia of Hot Climates, caused by the presence of Bilharzia Hæmaturia. By F. H. H. GuILlemard, M.A., M.D., F.R.G.S. Price 2s.

Fever. Text-Book of the Eruptive and Continued Fevers. By ЈонN William Moore, B.A., M.D., M. Ch. Univ. Dub., F.R.C.P.I., Professor of Practice of Medicine in the Schools of the Royal College of Surgeons in Ireland, Physician to the Meath Hospital, Consulting Physician to Cork Street Fever Hospital, Dublin. Price 15s.

Fever Charts. Daily Clinical Fever Charts, to record the progress of a case of fever. By F. MAgee Finny, M.D. Price 5s.

Food. Aids to the Analysis of Food and Drugs. By H. AUBREY Husband, M.B., F.R.C.S., Lecturer on Public Health in the Edinburgh Medical School. Price 1s. 6d. cloth; 1s. paper.

Food. The Healthy Manufacture of Bread. By B. W. Richardson. M.D., F.R.S. Price 6d. paper cover ; cloth, 1s., with Vignette.

Food. The Spirit of Cookery : a Popular Treatise on the History, Science, Practice, and Medical Import of Culinary Art; with a Dictionary of Culinary Terms. By J. L. W. ThudichuM, M.D., F.R.C.P. London. Price 6s.

Foot. Movable Atlas of the Foot; its Bones, Muscles, etc. By Prof. Witkowski. Price 7s. 6d. (See Anatomy.)

Foot. Deformities of the Foot. By W. J. WalshaM, M.B., F.R.C.S. Eng., Surgeon to St. Bartholomew's Hospital. Profusely illustrated. Price 18s.

Foot. A Practical Treatise on Orthopædic Surgery. For the use of Practitioners and Students. By James K. Young, M.D. Instructor in Orthopædic Surgery, University of Pennsylvania. Price 18s.

Forensic Medicine. The Maybrick Case. A Treatise by A. M. MACDOUgall, B.A., LL.D. Price 10s. 6d.

Forensic Medicine. The Student's Handbook of Forensic Medicine and Public Health. By H. Aubrey Husband, M.B., F.R.C.S.E. Sixth edition. Price 10s. 6d. net.

Forensic Medicine. Aids to Forensic Medicine and Toxicology. By WM. MurRell, M.D., F.R.C.P. Lond., Physician to, and Lecturer on Materia Medica, Westminster Hospital. Sixth thousand. Price 2s. 6d.

Geology. Field Geology, with a Section on Palæontology. By W. Hy. Penning, F.G.S., of H.M. Geological Survey, and A. J. JukEs-Browne, B.A., F.G.S. Second edition, enlarged. Price $7 \mathrm{~s} .6 \mathrm{~d}$.

"Others have taught us the principles of the science, but Mr. Penning, as an accomplished field-geologist, introduces us to the practice." - The Academy. 
Geology. Engineering Geology. By the same Author. Illustrated with coloured maps and woodcuts. Price 3s. $6 \mathrm{~d}$.

"A full and lucid description of surveying and mapping, the diagncsing of the various minerals met with, the value of sites, rocks, etc."-Popular Science Revievo.

Gout. A Treatise on Gout. By Austin MeLdon, M.K.Q.C.P., F.R.C.S.I., Senior Surgeon Jervis Street Hospital, Corisulting Physician Dublin General Infirmary. Tenth edition. Price 2s. 6d.

Gout. The Nature and Treatment of Gout. By Profesisor EissteIN of Gottingen University. Translated by J. E. BuRTon, L.R.C.P. Lond. Price 3s. $6 \mathrm{~d}$.

Gout. Notes and Conjectures on Gout and certain Allied Diseases. By Dr. Mortimer Granville. Price 1s. net.

Gynæcology. The Diseases of Women and their Treatment. By H. Macnaughton Jones, M.D., F.R.C.S.I., F.R.C.S.E., Examiner in Midwifery, Royal College of Surgeons, Ireland. Sixth edition. Illustrated, price 12s. 6d.

"A storehouse of information."-The Lancet.

"The work of a mature and experienced authority."-British Medical Journal.

"Of exceptional merit drawn from a field of wide personal experience."-Medical Press.

Gynæcology. Clinical Gynæcology: being a Handbook of Diseases peculiar to Women. By Thos. More MadDen, M.D., F.R.C.S. Ed., Obstetric Physician and Gynæcologist, Mater Misericordiæ Hospital, Dublin. Price 12s. 6d.

Gynæcology. Aids to Gynæcology. By AlFred S. GubB, M.D. Paris, M.R.C.S., L.R.C.P., D.P.H., Obstetric Assistant and Gold Medallist Westminster Hospital. Second edition, enlarged. Cloth, 2s. 6d., and 2s. sewn.

Gynæcology. On some Symptoms which Simulate Disease of the Pelvic Organs in Women, and their Treatment by Albo-PiestoMyo-Kinetics and Auto-Piesto-Myo-Kinetics (Massage, Selfmovements of Muscles under Pressure). By A. C. F. Rabagliati, M.A., M.D., F.R.C.S. Price 7s. 6 d.

Hair. The Hair : its Growth, Care, Diseases, and Treatment. By C. H. LEONARD, A.M., M.D. Illustrated, price $7 \mathrm{~s} .6 \mathrm{~d}$.

Hair. A Synopsis of Diseases of the Skin and Hair. By R. GLASGowPatteson, M.B., Surgeon to St. Vincent's Hospital. Price 1s.

Hand. Movable Atlas of the Hand; its Bones, Muscles and Attachments. By Prof. Wiтkowski. Price 7s. 6d. (See Anatomy.) Hay Fever: its Causes, Treatment, and Effective Prevention; Experimental Researches. By Chas. Harrison BlaCkLEY, M.D. Second edition, revised and enlarged. Price 10s. 6d.

Heart. Heart-Studies, Chiefly Clinical. By WM. EwART, M.D. Cantab., F.R.C.P., Physician to St. George's Hospital; Examiner in Medicine at the Royal College of Physicians.

I. -The Pulse-Sensations. A Study in Tactile Sphygmology. Price 15s. 
Heart. An Essay on Fatty Heart. By Henry Kennedy, A.B., M.B. Physician to the Whitworth Hospitals. Price 3s. 6d.

Heart. Clinical Diagrams, with Directions for Recording Cases of Heart Disease. By Geo. Herschell, M.D. Lond. Price 1s.

Heredity and Disease. From Generation to Generation. By Douglas LithGow, LL.D., M.R.C.P. Lond. Price 4s. 6d.

Hernia and Intestinal Obstruction. By J. Roche, M.D. $6 \mathrm{~d}$.

Histology. Manual for the Physiological Laboratory. By VINCENT D. Harris, M.D., F.R.C.P., and D'ARCY Power, M.B. Oxon., Examiners on the Conjoint Board. Fifth edition. Price 7s. 6d.

Histology. Introduction to Practical Histology. By George ThIN, M.D. Price 5s.

Histology. Text-book of Normal Histology : including an Account of the Development of the Tissues and of the Organs. By Professor Geo. A. Piersol, M.D. Price 15̃s.

Histology. The Microscopical Examinations of the Human Brain; Methods of Research, etc. By EDwIN Goodall, M.D. Lond., Pathologist to the West Riding Asylum. Price 5s.

History of the Royal College of Surgeons in Ireland. By Sir C. A. Cameron. Price 10s. $6 \mathrm{~d}$.

Hydatid Disease, with special reference to its prevalence in Australia. By J. Davis Thomas, M.D. Lond., F.R.C.S. Eng. Edited by A. B. Landon, M.D. Lond. Price 10s.

A Collection of Papers on Hydatid Disease. Being Part II. of the above. By A. B. Landon. 5s.

Hydrophobia. Comments on the Reports of the Committee on M. Pasteur's Treatment. By Surgeon-General C. A. GoRDoN, M.D., C.B. Price 2s. $6 \mathrm{~d}$.

Hydrophobia. Inoculation for Rabies and Hydrophobia. A Study of the Literature of the subject. Price 2s. 6d.

Hydrophobia. An Address upon Hydrophobia, Dog-bites, and other Poisoned Wounds. By C. R. ILLINGWORTh, M.D. Price 1s.

Hydropathy, or the Practical Use of Cold Water. By E. MarLETT BODDY, F.R.C.S., F.S.S., L.R.C.P. Price 1s.

Hydropathy. Notes of Visits to Contrexéville and Royat-lesBains. By F. R. Cruise, M.D. Price 6d.

Hydropathy. Vichy and its Therapeutical Resources. By Prosser JAMES, M.D., M.R.C.P. Lond. Price 2s. $6 \mathrm{~d}$.

Hygiene. Lessons in Military Hygiene and Surgery, from the Franco-Prussian War. Prepared on behalf of Her Majesty's Government. By Surgeon-General GoRDox, M.D., C.B., Hon. Physician to the Queen. Illustrated, price 10s. 6d. 


\section{Baillière, Tindall, and Cox's Books.}

Hygiene. A Manual of Sanitation; or, First Help in Sickness and when Wounded. Alphabetically arranged. By the same Author. Cloth, 2s. 6d.; sewn, 1s.

Hygiene. Nature's Hygiene. A Manual of Natural Hygiene. By C. T. KingzetT, F.I.C., F.C.S. Fourth Edition. Price 10s.

Hygiene. The Elements of School Hygiene for the Use of Teachers and Schools. By W. E. Roth, B.A. Price 3s. 6d.

Hygiene. Theatre Hygiene, a study in construction, safety and healthy arrangement. By W. E. Roth, B.A. Oxon. Price 1s. 6 d.

Hygiene. Healthy Homes. By Stanley Haynes, M.D., M.R.C.S., F.R.G.S. Price 1s.

Hygiene. Notes on Nuisances, Drains, and Dwellings. By W. H. Penning, F.G.S. Second edition. Price 6d.

Hygiene. Short Lectures on Sanitary Subjects. By Richard J. Halton, L.K.Q.C.P., L.R.C.P. Ed., L.R.C.S.I., etc. Price 5s.

Hygiene. A Manual of Naval Hygiene, with Instructions and Hints on the Preservation of Health and the Prevention of Disease on board Ship. By Joseph Wilson, M.D. Second edition. 10s. $6 \mathrm{~d}$.

Hygiene. The Sanitation of Public Institutions. The Howard Prize Essay. By R. D. R. Sweeting, M.R.C.S., Medical Superintendent of the Western Fever Hospital. Price 3s. 6d.

Hygiene. Hygiene of Beauty, with Details of the Cosmetic Art, Prescriptions for the Skin, Teeth, Mouth, Hair, etc., and Formulas for Perfumes. By Dr. Monin. Translated by B. CARDWELl. Price 3s. 6d.

Hypnotism. Psycho-Therapeutics. Treatment by Hypnotism and Suggestion. By J. LLOYD TuCKEY, M.D. Third edition, enlarged. Price 6s.

Indigestion. Indigestion: An Introduction to the Study of Diseases of the Stomach. By George Herschell, M.D. Lond. Second edition. Price 5s. net.

Infection. Creameries and Infectious Diseases. By J. J. WeLPLy, M.D., M.Ch., Q.N.I. Second Edition. Price 1s.

Inflammation. The State of the Blood and the Bloodvessels in Inflammation. By T. WharToN JoNes, F.R.C.S., F.R.S., Emeritus Professor of Ophthalmic Medicine and Surgery in University College, London. Price 2s. 6d.

Insanity. Handbook for Attendants on the Insane. Prepared by Authority of the Medico-Psychological Association. Price 2s. 


\section{Baillière, Tindall, and Cox's Books.}

International Medical Congress. The Commemorative PortraitPicture of the International Medical Congress, 1881. Designed and executed by Mr. BARRAUD ; nearly 700 Likenesses of Members, representing Medicine and Surgery in every part of the world; special sittings accorded for every Portrait.

The Picture is Printed by the Newo Permanent Carbon Process in troo Sizes:

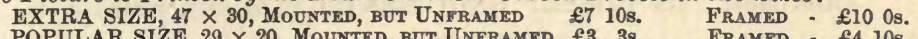

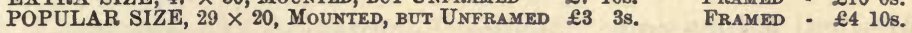

Kidneys. Vaso-Renal Change versus Bright's Disease. By J. MILNER FothERGILL, M.D. Ed. Price 7s. 6d.

Kidneys. Bright's Disease of the Kidneys. By Professor J. M. Charcot. Translated by H. B. Miluard, M.D., A.M. Revised by the Author, with coloured plates, price 7s. $6 \mathrm{~d}$.

Kidneys. Movable Kidney and Intermitting Hydronephrosis. By G. D. KNight, M.D. Price 3s. 6d.

Materia Medica. A Dictionary of Materia Medica and Therapeutics. A Résumé of the Action and Doses of all Officinal and Non-officinal Drugs now in Common Use. By C. HeNri LeoNaRD, A.M., M.D., and Thos. Christy, F.L.S., F.C.S. Price 6s. ; half morocco, $8 \mathrm{~s} .6 \mathrm{~d}$.

The authors claim to have incorporated everything of merit that could be found either in standard works or manufacturers' catalogues. The scheme embraces the Pronunciation, Genitive case-ending, Common Name, Dose, and Metric Dose. Then the Symonyms, English, French, and German. If a Plant the Part Used, Habitat, Natural Order, and Description of Plant and Flowers, with its Alkaloirls, if any. If a Mineral, its Chemical Symbol, Atomic Weight, looks, taste, how found, and its peculiarities. Then the Action and Uses of the Drug, its Antagonists, Incompatibles, Synergists and Antidotes. Then follow its Officinal and Nonofficinal preparations, with their Medium and Maximum Doses. A handy volume for either the Physician, Student, or Druggist.

"Will, we are sure, fulfil a long-felt want."-British und Colonial Druggist.

"Well up to date. - . - Contains an index of great value."-Chemist and Druggist.

Materia Medica. Table of Doses. By J. H. Allan, F.C.S. Price 6d., cloth.

Materia Medica. Aids to Materia Medica and Therapeutics. By C. E. ARMand Semple.

Part I.-The Non-metallic and Metallic Elements, Alcoholic and Ethereal Preparations, etc. Cloth, 2s. 6d.; paper, 2s.

Part II.-The Vegetable and Animal Substances. 2s. 6d., 2s.

Part III.-Classification of Remedies. Cloth, 1s. 6d. ; paper, 1s.

Part IV.-New Remedies of the British Pharmacopoeia. Cloth, 2s. 6d. ; paper, 2 s.

Part V.-Tablets of Materia Medica. Price, cloth, 1s.6d.; paper, 1s. Materia Medica and Pharmacy. A Text-Book for Medical and Pharmaceutical Students preparing for Examination. By W. HANDsel Griffiths, Ph.D., F.C.S., F.R.C.P. Ed. Third edition. Edited by A. S. GUBB, M.D. Paris, L.R.C.P. Lond., M.R.C.S., D.P.H., Gold Medallist, Prizeman in Materia Medica, Westminster Hospital. Price 7s. 6d.

"A book of great value . . a standard text-book."-Edin. Med. Joumal.

"One of the ablest, if not the best, work on the subject in our language." - Med. Press. 
Materia Medica. Notes on Inorganic Materia Medica, and its Chemistry. By J. S. Sharman. Second edition. Price 1s. 6d.

Materia Medica. Notes on Materia Medica and Therapeutics. Mineral Drugs, Part I. By J. S. McArdle. Price 1s.

Medical Charities. The Reform of Our Voluntary Medical Charities. By Robert Reid Rentoul, M.D. Price 5s.

Medical Education. Medical Education and Organization. The Hunterian Oration for 1880 . By Walter Rivington, B.A., M.B., F.R.C.S., Surgeon to the London Hospital. Price 1s.

Medical Etiquette. A Few Rules of Medical Etiquette. By a L.R.C.P. Lond. Price 1s.

Medical Jurisprudence. (See Forensic Medicine.)

Medical Laws. Medical Law for Medical Men: their Legal Relations popularly explained. By Professor Meymotr TIDY, M.B., F.C.S., Barrister-at-Law, and Percy Clarke, LL.B., Solicitor. Leather, gilt edges, price $4 \mathrm{~s}$.

Medical Laws. The Laws Relating to Medical Men. By James Greenwood, Barrister-at-Law. Price 5s.

"Admirably suited as a guide to the busy practitioner, who frequently runs great risks of becoming involved in legal penalties, in consequence of an imperfect knowledge of the law." -Glasgow Medical Journal.

Medical Profession. A Guide to the Medical Profession in all its branches, including the Public Services. By C. R. B. Keetley, F.R.C.S. Second edition. Price 3s. 6d.

Medical Profession. Medical Men and Manners of the Nineteenth Century. By a Physician. Third Thousand. Price 3s.

"At times scathing, at others amusing, the author is never dull, and writes as one who knows the many blots on our system, and honestly tries to remedy them."-Medical Press.

Medicine. Dictionary of Medicine. (See p. 18.)

Medicine. Aids to Medicine. By C. E. Armand Semple, B.A. M.B. Cantab., M.R.C.P. Lond.

Part I.-General Diseases. Price 2s. 6d. and 2s.

Part II.-The Urine, Kidneys, Stomach, Peritoneum, Throat, and CEsophagus. Third Thousand. Price 2s. 6d. and 2s.

Part III.-Diseases of the Brain, Nervous System, and Spinal Cord. Third Thousand. Price 2s. 6d, and 2s.

Part IV.-Fevers, Skin Diseases. Price 2s. 6d. and 2s.

Medicine. A Chronolog ${ }_{v}$ of Medicine from the Earliest Times. By J. Morgan Richards. Price 10s. 6 d.

Medicine. Student's Handbook of the Practice of Medicine. By H. Aubrey Husband, M.B., C.M., B.Sc. Price 7s. 6d.

Medicinal Remedies. Notes on Medicinal Remedies. By J. P Stephenson. Price 1s. 6d. 
Medico-Military Services. Our Services under the Crown. A Historical Sketch of the Army Medical Staff. By Surgeon-Major A. Gore, M.D., Sanitary Officer on the Staff. Price 6s.

Memory. Its Logical Relations and Cultivation. By F. W. EDRIDGE-Green, M.D., F.G.S., Author of "Colour Blindness." Second edition. Price 6s.

Mental Medicine. A Practical Manual of Mental Medicine. By Dr. E. Regis, translated by Professor M. Benjamin Hall. Price 10s.

Meteorology. The Moon and the Weather: the Probability of Lunar Influence Reconsidered. Showing how storms and depressions may be predicted. By WALter J. Browne (St. Petersburg). Second edition. Price 3s.

Microscopical Science. Modern Microscopy. A Handbook for Beginners. The Microscope, and Instructions for its Use. Microscopic Objects: How Prepared and Mounted. By M. I. Cross and Martin J. Cole. Second edition in preparation.

Midwifery. (See Obstetrics.)

Mineral Waters. The Mineral Waters of Europe. A complete Analytical Guide to all the Bottled Waters, and their Medicinal and Therapeutic Values. By Professor TichBorne, LL.D., and M. Prosser James, M.R.C.P. Lond. Price 3s. 6d.

"Such a book as this is simply invaluable."-The World.

Morals. A Physician's Sermon to Young Men. By William PratT, M.A., M.D., etc. Eighth thousand. Price 1s. cloth.

"The delicate topic is handled wisely, judiciously, and religiously, as well as very plainly." -The Guardian.

Morals. Revelations of Quacks and Quackery. With Facts and Cases in Illustration of their Nefarious Practices. By "DETECTOR." Thirtieth thousand. Price $2 \mathrm{~s}$.

Morphia. On the cure of the Morphia Habit. By Oscar Jennings, M.D. Paris, F.R.C.S. Eng. Price 2s. 6 d.

Nerve Supply. Atlas of Cutaneous Nerve Supply. By JACOB Heiberg, M.D., and W. W. WagstafFe, F.R.C.S. Containing 10 plates in colours. Price 4 s. 6 d.

Nervous Diseases. Functional Nervous Diseases, their Causes and Treatment. By Geo. T. Stevens, M.D., Ph.D. With plates. Price 12s.

Nervous Diseases. Clinical Notes on Nerve Disorders in Surgical Practice. By Geo. Wherry, M.A., M.S. Cantab., F.R.C.S. Price 2s.

(See also Brain.) 
Neuralgia. The Surgical Treatment of Neuralgia of the Fifth Nerve; being the Lettsomian Lectures for 1892. By WM. Rose, M.B., B.S. Lond., F.R.C.S., Joint Professor of Surgery in King's College, London, and Surgeon to King's College Hospital. Illustrated. Price 4s. 6d.

Nose. A Handbook of Diseases of the Nose and Pharynx. By JAS. B. BALL, M.D. (Lond.), M.R.C.P., Physician to the West London Hospital. Second edition. Price 7s. 6d.

Nursing. Handbook for Attendants on the Insane. Published by the authority of the Medico-Psychological Association. With illustrations and questions for examinations. Price 2s.

Nursing. Handbook of Obstetrical and Gynæcological Nursing, being the fifth edition of "A Manual for Midwives." By the late Dr. Fleetwood Churchill. Revised and greatly enlarged by Thos. More Madden, M.D., F.R.C.S. Eng. With numerous illustrations. Price $4 \mathrm{~s}$. net.

Nursing. Questions and Answers on Nursing, for St. John Ambulance Associations, Nursing Institutes, and Nurses generally. By John W. Martin, M.D., Author of "Ambulance Work." Fourth thousand. Price 1s. 6d. net.

Nursing. How to Feed an Infant. With an Appendix on theCommon Ailments of Infancy, with their Hygienic and Curative Treatment. By Benson Baker, M.D. Price 1s. 6d.

Nursing. How to bring up Children by Hand. By J. Foster PALMER, L.R.C.P. Price $6 \mathrm{~d}$.

Nursing. Practical Guide for the Young Mother. From the French of Dr. BRochaRD, Director-General of Nurseries and Crêches, with Notes and Hints by a London Physician. Price 2s.

Obstetrics. Aids to Obstetrics. By Samuel Nall, M.B. Cantab., M.R.C.P. Lond., First Class Honours Nat. Sci. Cambridge, late Resident Obstetric Assistant, St. Bartholomew's Hospital. Price 2s. 6d. cloth; 2s. paper wrapper.

Obstetrics. Hints for Midwives on Pregnancy and Labour. Abstracts of a Series of Lectures by H. MACNAUGHTON JONES, M.D., M.C.H., F.R.C.S. Price 1s. net.

Obstetrics. The Diagnosis and Treatment of Extra-uterine Pregnancy. By JoHn Strahan, M.D., M.Ch. (The Jenks Triennial Prize Essay awarded by the College of Physicians, 1889.) Price 4s. 6d.

Obstetrics. Hints for the Use of Midwives preparatory to their Examinations. By R. J. M. Coffin, F.R.C.P. Ed. Second Edition, enlarged. Price 2s. 


\section{Baillière, Tindall, and Cox's Books.}

Odontology. (See Dental.)

Old Age. The Diseases of Sedentary and Advanced Life. By J. Milner Fothergill, M.D., M.R.C.P. Lond. Price 7s. 6d.

Ophthalmology. (See Eye.)

Osteology. Osteology for Students, with Atlas of Plates. By ARThUR Trehern Norton, F.R.C.S., Surgeon to, and Lecturer on Surgery at, St. Mary's Hospital. Atlas and Text in one volume, 7 s. $6 \mathrm{~d}$. ; in two volumes, $8 \mathrm{~s}$. $6 \mathrm{~d}$.

"The handiest and most complete handbook on Osteology." - The Lancet.

Osteology. Atlas of the Skeleton and its Articulations, showing the Bones and Ligaments of the Human Body and Limbs. By Professor WrTkowski. Price 7s. 6d. (See Anatomy.)

Overwork. Overwork and Premature Mental Decay : its Treatment. By C. H. F. RouTH, M.D., M.R.C.P. Lond. Fourth edition. Price 2s. $6 \mathrm{~d}$.

Pathology. Lectures on Medical Pathology. By H. G. SutTon, M.B., F.R.C.P. Lond., late Physician to, and Lecturer on Pathology at, the London Hospital. Price 5s.

" Such a work is to be accepted with gratitude for the thoughts it contains, and the facts on which they are based."-The Lancet.

Pathology. Handbook of Medical Pathology for the use of Students in the Museum. By Drs. Herringham, Garrod, and Gow, of St. Bartholomew's Hospital. Price 7s. 6d.

Pathology. Handbook of Surgical Pathology. Edited by W. J. WaLSHAM, M.B., F.R.C.S., and D'ArCY Power, M.B. Oxon., F.R.C.S. Second edition. Price 9s.

"An embodiment of the most modern pathological teaching."-The Lancet.

Pathology. Aids to Pathology. By Gilbert A. Bannatyne, M.D. General : Cloth, 1s. 6d. sewn, 1s. Special: Cloth, 2s. 6d.; sewn, 2 s.

Pathology. Illustrations of Pathological Anatomy issued in parts, each containing 4 plates in colours, with descriptive text by Professors Kast, of Breslau, and RUMPEL, of Hamburg. The English edition revised and edited by $M$. ARMand RUFFer, M.D. Oxon. Twelve parts by subscription, post free, $£ 28$ s. Single parts, 6s. each. Single plates, 1s. 6d. each.

Pathology. Examination Cards. Arranged as questions and answers for self-examination. By A. T. ScHoField, M.D., M.R.C.S. Complete in two sets of cards, price $9 \mathrm{~d}$. net per set. Mr. Jonathan Hutchinson, F.R.C.8., writes : "It is an invaluable means of self-tuition."

Peritonitis. Localised Peritonitis: its Etiology, Diagnosis, and Treatment. By JoHn Wallace, M.D., Professor of Midwifery in the Victoria University. Illustrated. Price 1s. 
Pharmacopœia. A Vest-Pocket Epitome of the British Pharmacopœia. By Russell Coombe, M.A., F.R.C.S. Cloth, price 1s.

Pharmacopœia. The Pocket Pharmacopœia. A Précis of the British Pharmacopoeia, including the Therapeutical Action of the Drugs, their Natural Orders and Active Principles. By C. ARMAND SEMPLE, M.D., M.R.C.P. Second edition, with the Appendix of 1890 . Price $3 \mathrm{~s} .6 \mathrm{~d}$.

Pharmacopœia. Notes on the Pharmacopœial Preparations for Pharmaceutical Students. By HANDSEL GRIFFiths ; revised by A. S. GubB, M.D. Paris, L.R.C.P., M.R.C.S., D.P.H. Price 3s. 6d.

Pharmacy. Latin Grammar of Pharmacy, for the use of Students, with an Essay on Latin Prescriptions. By JosEPH INCE, A.K.C.L., formerly Examiner and Member of Council, Pharmaceutical Society. Sixth edition. Price 5s.

Pharmacy. Aids to Pharmacy. By C. E. Armand Semple, M.B. Cantab., M.R.C.P. Lond. Cloth, price 2s. 6d.; paper, 2s.

Pharmacy. Practical Pharmacy for Medical Students. By A. CAMPBELl Stark, Demonstrator on Materia Medica and Pharmacy at St. George's Hospital. Price 3s. 6d., or interleaved for note-taking, 4 s. $6 \mathrm{~d}$.

Pharmacy. Doses and Strengths of the British Pharmacopœia. By the Principals of the Middlesex College of Chemistry. Price 6d.

Phimosis. Its Causes, Symptoms, and Treatment; with a description of the ancient rite of circumcision. By L. H. ORMSBY, M.D., F.R.C.S.I., Lecturer on Clinical and Operative Surgery at, and Surgeon to, the Children's Hospital, Dublin. Price 1s.

Physics. A Manual of Physics. Being an Introduction to the Study of Physical Science designed for University Students. By W. PeddIE, D.Sc., F.R.S.E., Lecturer on Physics in the University of Edinburgh. (University Series of Manuals.) 7s. 6d.

"Altogether worthy of praise. . . . We have no hesitation in giving it high commenda. tion, feeling well satisfied that it meets a decided want."-Nature.

"Dr. Peddie's manual is deserving of the highest praise."-Lancet.

"Written with a precision of statement and clearness of exposition which does the writer infinite credit."-Dundee Advertiser.

"A very handy work of reference."-University Correspondent.

"The work must be pronounced highly satisfactory, creditable to author and publishers, and calculated to be useful to the readers for whom it is intended."-Chemical Neros.

"The book deserves an extended circulation."-Science and Art.

Physiological Chemistry. Aids to Physiological Chemistry. By J. L. Thudichum, M.D., F.R.C.P. Lond., St. Thomas's Hospital. Cloth, price 2s. $6 \mathrm{~d}$. Sewn, 2s.

Physiological Factor in Diagnosis. By J. Milner Fothergill, M.D., M.R.C.P. Lond., Physician to the City of London Hospital for Diseases of the Chest. Second edition. Price 7s. 6d. 


\section{Baillière, Tindall, and Cox's Books.}

Physiology. A Manual of Physiology. By G. N. Stewart, M.A. D.Sc. University of Cambridge. (University Series of Manuals.)

[In the Press.

Physiology. The Physiologist in the Household. By J. MrLner ForhergiLl, M.D., M.R.C.P. Part I._Adolescence. Price 1s.

Physiology. Aids to Physiology. By B. Thompson Lowne, F.R.C.S., Arris and Gale Lecturer, and Examiner in Physiology, Royal College of Surgeons of England. Fourth thousand, illustrated. In two parts, $2 \mathrm{~s}$. each, or in one vol., cloth, $4 \mathrm{~s}$. $6 \mathrm{~d}$.

" $\mathrm{As}$ 'aids' and not substitutes, they will prove of real valus to students." - MIedical Press. "Certainly one of the best of the now popular 'Aid Series." "-Students" Journal.

Plant Analysis. (See Chemistry.)

Polypus in the Nose and other Affections of the Nasal Cavity; their successful treatment. By J. L. W. Thudichum, M.D.

F.R.C.P. Lond. Seventh edition, enlarged. Price 2s. 6 d.

Population. On the Evils, Moral and Physical, likely to follow, if practices, intended to act as checks to population, be not strongly discouraged and condemned. By C. H. F. Routh, M.D., F.R.C.P. Second thousand. Price 1s.

Posology. Posological Tables: a Classified Chart, showing at a glance the Dose of every Officinal Substance and Preparation. By Handsel GrifftThs, Ph.D., L.R.C.P. Fifth edition, revised by PETER W. SQuire, F.L.S., F.C.S. Price 1s. ; or mounted on linen, rollers, and varnished, 3s. $6 \mathrm{~d}$.

Pregnancy. (See Obstetrics.)

Prescriptions. The Student's Pocket Prescriber. By H. AUbrey Husband, M.B., F.R.C.S.E. Price 1s. cloth.

Psychological Medicine in John Hunter's Time and the Progress it has made. By Fletcher Beach, M.B., F.R.C.P. Price $1 \mathrm{~s}$.

Psycho-Therapeutics. (See Hypnotism.)

Public Health. Aids to Sanitary Science, for the Use of Candidates for Public Health Qualifications. By F. J. Allan, M.D., Dipl. Public Health, Camb., Assistant Professor of Hygiene, Coliege of State Medicine. Price 4s. 6d. cloth.

"A really admirable synopsis of what it is most necessary for a candidate to know."Glasgoro Medical Journal.

"The information contained is correct, well expressed and well arranged."-Public Health.

"The work has been well done. . . . Will be found a serviceable and reliable aid."Bdinburgh Medical Journal.

Public Health. The Practical Guide to the Public Health Acts and Correlated Acts for Officers of Health and Inspectors of Nuisances. By Thos. Whiteside Hime, B.A., M.B. Second edition, enlarged. Price

Public Health. Aids to Public Health. By J. L. Thudichum, M.D., F.R.C.P. Lond. Price 1s. 6d. cloth; 1s. paper. 
Public Health. Guide to Sanitary Science Examinations. By Herbert Jones, D.P.H. Cantab. Price 2s. 6d.

Pulse. How to feel the Pulse and what to Feel in it. Practical Hints for Beginners. By William Ewart, M.D., F.R.C.P. Lond., Physician to St. George's Hospital. With a glossary and twelve illustrations. Price 3s. 6d.

Pulse. The Sphygmograph : its History and uso as an aid to Diagnosis. By R. E. Dudgeon, M.D. Price 2s. 6 d.

Rabies. (See Hydrophobia.)

Respiration. Keep your Mouth Shut; a Popular Treatise on Mouth-breathing. By Fred. A. A. SMith, M.D., C.M. Glas. Price 2s. 6d.

Rheumatism. Its Treatment by Electric Massage, etc., in connection with the Wiesbaden Thermal Waters. By CARL Mordhorst, M.D. Kiel. Price 1 s.

Rupture of the Perineum. Its Causes Prevention and Treatment. By Michael Joseph Molony, M.R.C.P., L.R.C.S. Price 2s. cloth, 1s. 6d. paper.

Salt. History of Salt, with Observations on its Medicinal and Dietetic Properties. By Evan Marlett Boddy, F.R.C.S., F.S.S., L.R.C.P. Price 2s. 6 d.

Sewage. The Sewage Question: Reports upon the Principal sewage Farms and Works of the Kingdom, with Notes and Chemical Analyses. By the late Dr. Letheby. Price 4s. 6d.

Skin Diseases of Infancy and Early Life. By C. M. CAMPBELL, M.D., C.M. Edin. Price 5s.

Skin. A Synopsis of Diseases of the Skin and Hair. By R. Glasgow Patteson, M.B., Surgeon to St. Vincent's Hospital. Price 1s.

Skin. Dermic Memoranda : An Introduction to the Study of Skin Disease, with Special Reference to the Exanthemata. By William Gemmel, M.B., Glasgow Fever Hospital. Price 3s. net.

Skin. Some Diseases of the Skin produced by Derangements of the Nervous System. By T. STRETCH Dowse, M.D., F.R.C.P.E. Price 2s.

Stomach. The Surgical Diseases and Injuries of the Stomach and Intestines. By F. Bowreman JesseTt, F.R.C.S., Surgeon to the Cancer Hospital. Numerous engravings. Price 7s. 6d. 


\section{Baillière, Tindall, and Cox's Books.}

Stricture. Stricture of the Urethra: its Diagnosis and Treatment. By E. Distin MadDick, F.R.C.S. Edin., late Surgeon R.N. 4s.

Surgery. The Rules of Aseptic and Antiseptic Surgery. By A. G. Gerster, M.D., Professor of Surgery at the New York Polyclinics. With 248 engravings and 3 coloured plates. Price 15s.

Surgery. The Science and Practice of Surgery, a Complete Textbook. By F. J. GANT, F.R.C.S., Senior Surgeon Royal Free Hospital. Third edition, nearly 1,100 engravings. 2 vols., price 36 s. Surgery. The Student's Surgery : a Multum in Parvo. By F. J. GANT, F.R.C.S. 850 pp., illustrated. Price 10s. 6 d.

Surgery. Operative Surgery on the Cadaver. By JASPER J. Garmany, A.M., M.D., F.R.C.S. Price 8s. 6d.

Surgery. Aids to Surgery. By GEorge Brown, M.R.C.S. 2 parts, price 1 s. 6 d. cloth, and 1s. sewn, each; or in 1 vol., 2 s. $6 \mathrm{~d}$.

Surgery. The Text-hook of Operative Surgery. With 88 beautifully engraved steel plates, after BERNARD and HUETTE. Text by Arthur Trehern Norton, F.R.C.S., Surgeon to, and Lecturer on Surgery at, St. Mary's Hospital. Second edition, half calf, plain, $25 \mathrm{~s}$.; hand-coloured, 50 s.

Surgery. Clinical Lectures on Recent Surgery. By ARTHUR Trehern Norton, F.R.C.S. Price 3s.

Surgery. The Anatomy of Surgery. By John McLachlan, M.B., M.K.C.S. With 74 illustrations. Two vols., price $18 \mathrm{~s}$.

Surgery. The Surgery of the Knee-Joint, and the Responsibility placed on the Physician and General Practitioner by the Modern Process of Surgery. By C. B. KeETley, F.R.C.S., Senior Surgeon to the West London Hospital, and Surgeon to its Orthopædic Department. Cloth, price 1s. 6d.

Surgery. Brain Surgery. By M. Allen Starr, M.D. With 59 illustrations. Price 10s. 6 d.

Surgery, Minor-and Bandaging. Questions and Answers for Self-examination. By A. T. Schofield, M.D. Price 9d. net.

Surgical Pathology. Handbook of Surgical Pathology. By W. J. WalshaM, M.B., F.R.C.S., and D'Arcy Power, M.B., F.R.C.S. Second edition. Price 9s.

"An embodiment of the most modern pathological teaching."-The Lancet.

Surgical Anatomy. (See Surgery.)

Surgical Treatment. Notes on Surgical Treatment and Minor Operations. Designed especially for House Surgeons and Students. By T. F. HoPGOod, L.R.C.P., M.R.C.S. Surgeon to the Sunderland Infirmary. Price 2s. 6d. 


\section{Baillière, Tindall, and Cox's Books.}

Syphilis. Syphilis of the Brain and Spinal Cord, showing the part which this agent plays in the production of Paralysis, Epilepsy, Insanity, Headache, Neuralgia, Hysteria, and other Mental and Nervous Derangements. By T. STRETCH Dowse, M.D., F.R.C.P. Ed. Second edition, illustrated. Price 5s.

Syphilis. The Nature and Treatment of Syphilis, and the other socalled Contagious Diseases. By C. R. DRYSDALE, M.D., M.R.C.P. Lond., F.R.C.S. Eng. Fifth edition. Price 5s.

Temperature Charts for Recording the Range of Temperature, Pulse, Respiration, History, Progress, and Treatment of Cases. By E. W. Moore, M.D., M.R.C.P. Price 1d. each, 9d. per dozen ; or mounted, similar to a blotting-pad, 50, 3s. 6d.; 100, 7s.

Theories of Life. The Protoplasmic Theory of Life. By JoHN Drysdale, M.D., F.R.M.S. Price 5s.

Theories of Life. How to Prolong Life. Showing the Diel and Agents best adapted for a lengthened prolongation of existence. By C. W. De Lacy Evans, M.R.C.S. Second edition. Price 5s.

"A good account of the changes which occur with the advance of age."-Lancet.

Therapeutics. Modern Therapeutics. Medical and Surgical. By Geo. H. Napheys, A.M., M.D. Ninth edition. Revised and enlarged by Drs. Allen Smith and Aubrey Davis.

Vol. I.-General Medicine, and Diseases of Children. Price, half morocco, $£ 110$ s.

Vol. II.-General Surgery, Gynæcology, and Obstetrics. Price, half morocco, $£ 110$ s.

Therapeutics. The Therapeutics of the Respiratory Passages. By Prosser James, M.D., Lecturer on Materia Medica and Therapeutics at the London Hospital. Price 10s. 6 d.

"Dr. Prosser James has produced a scholarly treatise." - New York Medical Record.

Therapeutics. Aids to Rational Therapeutics, for the guidance of Practitioners and Senior Students. By J. Milner Fothergill, M.D. Second edition. Price 2s. 6d. cloth; 2s. paper wrapper.

Throat. Movable Atlas of the Throat, and the Mechanism of Voice, Speech and Taste. By Prof. Witkowski. (See Anatomy.)

Throat. Diseases of the Throat and Nose. A Practical Guide to Diagnosis and Treatment. With 220 typical illustrations in chromolithography and numerous wood engravings. By LenNox Browne, F.R.C.S. Edin., Senior Surgeon to the Central London Throat and Ear Hospital. New edition in preparation.

"One of the completest treatises on diseases of the throat in any language."-British Medical Journal.

"The best text-hook in the English lanquage."-Edinburgh Medical Journal. 


\section{Baillière, Tindall, and Cox's Books.}

Throat. Affections of the Throat and Larynx. By ARTHUR Trehern Norton, F.R.C.S., Surgeon to St. Mary's Hospital.

Second edition, illustrated. Price $6 \mathrm{~s}$.

"Short, simple, and thoroughly practical instruction."-Medical Press.

Throat. Tonsillitis in Adolescents. By C. HAIG-Brown, M.D.,

C.M., Medical Officer to the Charterhouse. Price 3s.

Transfusion. On Transfusion of Blood and Saline Fluids. By

C. Egerton Jennings, F.R.C.S. Third edition, with Preface by Sir Spencer Wells, Bart. Price 4s. 6d.

Tuberculosis. (See Consumption.)

Ulcers. Ulcers and their Treatment. For the use of Dressers. By W. S. Crawford, B.A. Cantab., F.R.C.S. Edinb. Price 1s.; cloth, 1s. 6 d.

Urinary Diseases. Diseases of the Bladder, Prostate Gland, and Urethra. By F. J. GANT, F.R.C.S., Senior Surgeon to the Royal Free Hospital. Fifth edition, enlarged. Price 12s. 6 d.

"The work throughout bears evidence of having been written by a thoroughly practical and experienced surgeon."-Lancet.

Urinary Surgery. Urinary Surgery of the present day contrasted with that of twelve years ago. By F. SwINFord EDwards, F.R.C.S. Price 1s.

Urine. The Urine in Health and Disease, and Urinary Analysis. By D. CAMPBELL BLACK, M.D., Professor of Physiology in Anderson's College, Glasgow. Price 7s. 6d.

Urine. The Urine; a Guide to its Practical Examination. By J. Tyson, M.D., Professor of Morbid Anatomy in the University, and President of the Pathological Society of Philadelphia. Seventh edition, with numerous illustrations. Price 7s. 6d.

"We think it the most practically useful guide we have on the subject."-Medical Record.

Vichy. Vichy and its Therapeutical Resources. By Prosser JAMes, M.D., M.R.C.P. Lond. Price 2s. 6 d.

Voice. The Philosophy of Voice. Showing the right and wrong Action of the Breath and Vocal Cords in Speech and Song. By Charles Lunn. Eighth edition. Price 5s.

Voice. Artistic Voice in Speech and Song. Dedicated to Mr. Sims Reeves and Mr. Santley. By the same Author. 1s.

Voice. The Voice Musically and Medically Considered. By C. Armand Semple, M.B. Cantab., M.R.C.P. Lond., Physician to the Royal Society of Musicians. Part I. Musical, price 1s. ; Part II., Medical, price 2s. ; or in one vol., cloth, 3s. 6d.

Whooping-Cough. Its Pathology and Treatment. Fothergillian Prize Essay. By Thos. M. Dolan, M.D., F.R.C.S.E. Price 3s.6d.

Zoology and Comparative Anatomy, Aids to. By MaJor GreENwOoD, M.D., Honours. Price 2s. 6d., and 2s. 


\section{THE STUDENTS' AIDS SERIES.}

Specially designed to assist Students in committing to memory and grouping the subjects upon which they are to be examined.

Aids to Analysis of Food and Drugs. By H. Aubrey Husband, M.B., F.R.C.S. 1s. 6d. cloth; 1s. paper.

Aids to Anatomy. By GEORge Brown, M.R.C.S., and P. MACLeOD YEARSLEY, F.R.C.S. 2s. 6d. cloth; 2s. paper.

Aids to Surgical Anatomy. By Eugene S. Yonge, M.B. Price 2s. 6d. cloth; 2s. paper.

Aids to Biology. Part I.-Elementary Tissues and Elementary Organisms (Protoza and Protophyta). By JOSEPH.W. WilliaMs. 2s. 6 d. cloth ; 2 s. paper wrapper.

Aids to Botany. By C. E. Armand Semple, B.A., M.B. Cantab., M.R.C.P. Lond., late Senior Examiner in Arts at Apothecaries' Hall. Third thousand. 2s. 6d. cloth; 2s. paper.

Aids to Chemistry. By the same Author.

Part I.-Inorganic: Non-Metallic Substances. 2s. 6d. cloth ; 2s. paper wrapper.

Part II.-Inorganic: The Metals. 2s. 6d. and 2s.

Part III.-Organic. Price, 2s. 6d. and 2s.

Part IV.-Tablets of Chemical Analysis. 1s. 6d. and 1s.

Aids to Practical Chemistry. Especially arranged for the Analysis of Substances containing a Single Base and Acid Radicle. By T. HuRd Gordon. 2s. 6d. cloth; 2s. paper.

Aids to the Diagnosis and Treatment of Diseases of Children. By JoHN McCAw, M.D., L.R.C.P. Price 3s. 6d. cloth; 3s. paper.

Aids to Dental Surgery. By Arthur S. Underwood, M.B., M.R.C.S., Lecturer on Dental Surgery at the Dental Hospital of London. 2s. 6d. cloth; 2s. paper.

Aids to Dental Histology. By the same Author.

Illustrated. 2s. 6d. cloth; 2s. paper.

Aids to Diagnosis. Part I.-Semeiological. By J. Milner FotherGILL, M.D., M.R.C.P. Lond. 1s. 6d. cloth ; 1s. paper.

Part II.-Physical. By J. C. Thorowgood, M.D., F.R.C.P. Lond. 1s. 6d. and 1s.

Part III.--What to Ask the Patient. By J. MILner Fothergill, M.D., M.R.C.P. Lond. 1s. 6 d. and 1s. The three in one vol., 3s. 6 d.

"A mine of valuable information."-Edinburgh Medical Journal. 


\section{Baillière, Tindall, and Cox's Books.}

Aids to Examinations. Being Questions and Answers on Materia Medica, Medicine, Midwifery, Pathology, etc. By D. WALSH, M.B., C.M., L.R.C.P. New edition. 2s. 6d. and 2s.

Aids to Forensic Medicine and Toxicology. By WM. MURrelL, M.D., F.R.C.P. Lond., Physician to Westminster Hospital. New edition. Price 2s. 6d. cloth; 2s. paper wrapper.

Aids to Gynæcology. By Alfred GuBB, M.D. Paris, D.P.H., Obstetric Assistant and Gold Medallist, Westminster Hospital. New edition. Cloth, 2s. 6d.; paper wrapper, 2s.

Aids to Materia Medica and Therapeutics. By C. E. Armand SEMPLE, B.A., M.B. Cantab., M.R.C.P. Lond.

Part I.-The Non-Metallic and Metallic Elements, Alcoholic and Ethereal Preparations. 2s. 6d. cloth; and 2s. paper.

Part II.-Vegetable and Animal Substances, 2s. 6d. and 2s.

Part III.-Classification of Remedies, 1s. 6d. and 1s.

Part IV.-New Remedies. 2s. 6d. and 2s.

Part V.-Tablets of Materia Medica. Price 1s. 6d. and 1s.

Aids to Medicine. By the same Author.

Part I.-General Diseases. Lungs, Heart, and Liver. Cloth, 2s. 6 d. ; paper, 2 s.

Part II.-The Urine, Kidneys, etc. 2s. 6d. and $2 \mathrm{~s}$.

Part III.-The Brain and Nervous System. 2s. 6d. and 2s.

Part IV.-The Fevers, Skin Diseases, etc. 2s. 6d. and 2s.

Aids to Obstetrics. By Samuel Nall, B.A., M.B. Cantab., M.R.C.P. Lond., late House Physician and Resident Obstetric Assistant, St. Bartholomew's Hospital. Twelfth thousand. Cloth, 2s. 6d. ; paper, 2s.

Aids to Ophthalmic Medicine and Surgery. By Jonathan Hutchinson, jun., F.R.C.S. Cloth, 2s. 6d.; paper, 2s.

Aids to Otology. By W. R. H. Stewart, F.R.C.S. Ed., Aural Surgeon to the Great Northern Hospital, etc. Price 2s. 6d. cloth ; 2s. paper wrapper.

Aids to Pathology. By Gilbert A. Bannatyne, M.D.

General-Cloth, 1s. 6d. ; paper wrapper, 1s.

Special-Cloth, 2s. 6d.; paper, 2s.

Aids to Pharmacy. By C. E. Armand Semple, B.A., M.B. Cantab., M.R.C.P. London. Cloth, 2s. 6d. ; paper, 2s.

Aids to Physiology. By B. Thompson Lowne, F.R.C.S., Examiner in Physiology, Royal College of Surgeons. Fourth thousand. In two parts, price $2 \mathrm{~s}$. each; or in one vol., cloth, $4 \mathrm{~s} .6 \mathrm{~d}$. "Certainly one of the best of the now popular Aids Series."-Students" Journal. 
Aids to Practical Physiology. By J. Brindley JAMes, M.R.C.S. Cloth, 1s. 6d. ; paper, 1s.

Aids to Physiological Chemistry. By J. L. Thudichum, M.D., F.R.C.P. Lond., formerly Lecturer on Physiological Chemistry, St. Thomas's Hospital. Cloth, 2s. 6d. ; paper, 2s.

Aids to Psychological Medicine. By Edwin Goodall, M.D. Lond.

[In preparation.

Aids to Public Health. By J. L. Thudichum, M.D., F.R.C.P. Lond. New edition. 1s. 6d. cloth; 1s. paper wrapper.

Aids to Sanitary Science for the Use of Candidates for Public Health Qualifications. By F. J. AlLAN, M.U., Assistant Professor of Hygiene, Coll. State Medicine. $236 \mathrm{pp}$. Cloth, 4s. 6d.; or in two parts, paper, 2s. each.

Aids to Surgery. In two parts. By George Brown, M.R.C.S. 1s. 6 d. cloth, and 1s. paper, each ; or in one vol., cloth, 2 s. $6 \mathrm{~d}$.

Aids to Rational Therapeutics. By J. Milner Fothergill, M.D., M.R.C.P. Lond. 2s. 6d. cloth ; 2s. paper.

Replies to Questions in Therapeutics. By BRINDLEY JAMES, M.R.C.S. Is. 6d. cloth; 1s. paper.

Aids to Zoology. By MAJOR Greenwood, M.D. Honours in Zoology, University of London. 2s. 6d. cloth; 2s. paper.

Aids to Mathematics of Hygiene. By R. Bruce Ferguson, M.A., M.B. Price 2s. 6d. ; 2 s.

\section{Aids to Analytical Geometry.}

The Straight Line and Circle. By A. Le Sueur, B.A. Cantab. Second edition. Price 2s.

The Conic Sections, with solutions of questions set at the London and other University Examinations. By GEORGE HEPPEL, M.A., St. John's College, Cambridge, Member of London Mathematical Society. Price 2s.

** A Catalogue of Standard French Works on Medicine, Surgery, and the Allied Sciences will be sent post free on application. 


\section{Baillière, Tindall, and Cox's Books.}

WORKS

ON

\section{VETERINARY MEDICINE AND SURGERY.}

Banham. Tables of Veterinary Posology and Therapeutics. With Weights, Measures, etc. By Professor George A. Banham, F.R.C.V.S. Price 2s. 6d.

Beacock. Prize Essay on the Breeding, Rearing, and Fattening of Cattle and Sheep, and proper treatment of Cows at time of Calving. By Joseph Beacock. Price 3d.

Courtenay. The Practice of Veterinary Medicine and Surgery. By E. Courtenay. Price 10s. 6 d.

"Written in a clear and concise style: will form a welcome addition to the library of the horse-owner, and those who take an interest in domesticated animals generally." -Mark Lane Bxpress.

Dhakmarvala. Demonstrations in the Modes of Handling and Examining the Horse. By NowroJi Dorabji Dhakmarvala, C.B.V.C., Lecturer on Anatomy and Physiology at the Government Veterinary College, Bombay. Price $4 \mathrm{~s}$.

Fleming. A Text-Book of Veterinary Obstetrics, including the diseases and accidents incidental to pregnancy, parturition and early age in the Domesticated Animals. By George Fleming, C.B., LL.D., F.R.C.V.S., F.R.G.S., late Principal of the Army Veterinary Department. Profusely illustrated. Second edition in preparation.

"Has filled up a void in a more satisfactory and complete way than any other member of his profession could have done."-The Field.

"No man who makes any pretensions to veterinary science or stock breeding can dispense with this work."-Live Stock Journal.

Parasites and Parasitic Diseases of the Domesticated Animals. A Treatise by L. G. Neumans, Professor at the National Veterinary School of Toulouse. Translated and Edited by Geo. Fleming, C.B., LL.D., F.R.C.V.S., with 365 illustrations. Price 25s.

"The value and importance of Neumann's Treatise cannot be over-estimated ; it is certainly the most scientific, interesting, and useful work that has graced veterinary literature for some years." - The Veterinary Journal.

"We do not hesitate to say that this is a work which all pathologists ought to possess : and the practitioner ... will not fail to add to his reputation if he has this book on his shelves." - The Lancet.

"This is one of the most useful of the many works with which Dr. Fleming has enriched English Veterinary literature. Although intended as a text-book for the veterinary student and practitioner, the translation has rendered it 80 readable that every intelligent farmer may derive a fund of useful information from its copiously illustrated pages."-Mark Lane Express.

"Cannot fail to be of immense value to both the veterinary profession and to British stock. breeders."-Bell's Weekly Messenger.

A Text-Book of Operative Veterinary Surgery. Part I. price 10s. 6d. Part II. in the Press. 


\section{Baillière, Tindall, and Cox's Books.}

Fleming. The Contagious Diseases of Animals : their.influence on the wealth and health of the nation. Price $6 \mathrm{~d}$.

- Animal Plagues; their History from the Earliest Times, Nature, and Prevention. Vol. I., to 1800 . Price 15s. - Vol. II., from A.D. 1800 to 1844 . Price 12s.

- On Roaring in Horses (Laryngismus Paralyticus). Its History, Pathology, and Treatment. With coloured plate and woodcuts. Price 6 s.

- Tuberculosis from a Sanitary and Pathological point of view. Price $1 \mathrm{~s}$.

- Human and Animal Variolæ. A Study of Comparative Pathology. Price 1s.

Practical Horse Shoeing. With 37 illustrations. 2s.

The Influence of Heredity and Contagion on the Propagation of Tuberculosis. By G. Fleming, F.R.C.V.S., Herr A. Lydtin, and M. Van Hertsen. Price 6s.

Gresswell. A Manual of the Theory and Practice of Equine Medicine. By J. Brodie Gresswell, F.R.C.V.S., and Albert GresswELL, M.R.C.S. Eng. Second edition, enlarged. Price 10s. 6 d.

\section{BY THE SAME AUTHORS.}

Equine Hospital Prescriber. Second edition. Price 2s. 6d.

Bovine Prescriber. Second Edition. Price 2s. 6d.

Veterinary Pharmacopoia. Materia Medica and Therapeutics. Price 10s. 6d.

Diseases and Disorders of the Horse. A Treatise on Equine Medicine and Surgery. Price 5s.

Hill. Principles and Practice of Bovine Medicine and Surgery, with woodcuts and coloured plates. By J. Woodroffe HiLL, F.R.C.V.S. New edition.

In preparation.

The Management and Diseases of the Dog. By J. W. HiLL, F.R.C.V.S. Third edition. Illustrated. Price $7 \mathrm{~s} .6 \mathrm{~d}$.

Hoare. Manual of Veterinary Therapeutics. By E. WALLIS HOARE, F.R.C.V.S. Price 10s. 6 d.

Lambert. The Germ Theory of Disease, Concisely and Simply Explained. By Colonel James LamberT, F.R.C.V.S., Army Veterinary Department. Price 1s.

Liautard. Manual of Operative Veterinary Surgery. By A. Liautard, M.D., F.R.C.V.S. 600 illustrations. Price 30s. Animal Castration. Price 7s. 6d.

Lameness of Horses and Diseases of the Locomotor Apparatus. Price 10s. 6d.

Lupton. Horses: Sound and Unsound, with the Law relating to Sales and Warranty. By James Irvine Lupton, F.R.C.V.S. Price 5s. 
Miller-Tellor. The Diseases of Live Stock, and their most efficient remedies. A Popular Guide for the Treatment of Horses, Cattle, Cows, Sheep, Swine, Fowls, Dogs, etc. By Wm. B. E. Mrluer, D.V.S., President of U.S. Veterinary Association, Willis P. Hazard, A. Liautard, M.D., F.R.C.V.S., and Lloyd V. TELlor, M.D. New edition. Price 10s. 6d.

McBride. Anatomical Outlines of the Horse. By J. A. McBride, Ph.D., M.R.C.V.S. Third edition. Illustrated. Price 8s. 6d.

Meyrick. Stable Management and the Prevention of Diseases among Horses in India. By J. J. MEYrICK, C.B., F.R.C.V.S., Superintendent of Horse Breeding for the Punjab. Price 2s. $6 \mathrm{~d}$.

Poyser. The Stable Management of Troop Horses in India. "The Collinsian" Prize Essay. By Major R. Poyser, À.V.D., F.R.C.V.S. Price 2s.

Reynolds. The Breeding, Rearing, and Management of Draught Horses. By Richard ReYnolds, M.R.C.V.S. Price 3s. 6d.

Robertson. A Handbook of the Practice of Equine Medicine. By Wy. Robertson, F.R.C.V.S., late Principal of the Royal Veterinary College, London. Second edition. Price 25s.

Smith. A Manual of Veterinary Hygiene. By Captain Frederick SmitH, A.V.D., M.R.C.V.S., Professor in the Army Veterinary School, Aldershot. Second edition. Price 10s. 6d.

"The work is a very solid one, and it is a pleasure to recommend it."-Army and Navy Mag.

"It should be on the bookshelf of every horseman, horsekeeper, and veterinary surgeon." - United Service Gazette.

Price

A Manual of Veterinary Physiology. By the same Author.

Second edition, enlarged, with additions and illustrations, in the Press.

"The work will commend itself to those for whom it was written by its conciseness and the able manner in which the important facts are dealt with and arranged."-Luncel.

"We offer our hearty congratulation to Captain Smith for his welcome contribution to our scanty professional literature."-Veterinary Journal.

"A valuable addition to the too small list of good veterinary text-books."-Journal of Comparative Pathology (MacFadyean).

"We do not remember to have met with a scientific book which is more readable; and it supplies one of the greatest wants in our literature."-Veterinary Record.

"This work ought to delight the heart of the veterinary student."-Nature.

Veterinary Diagrams in Tabular Form. With coloured and plain engravings. Size of sheet $28 \frac{1}{2}$ by 22 inches.

No. 1. - The External Form and Elementary Anatomy of the Horse. Price 3s. 6d., or mounted on roller ana varnished, 6s. $6 \mathrm{~d}$.

No. 2.-The Age of Domestic Animals. Price 2s. 6d., or mounted, $5 \mathrm{~s} .6 \mathrm{~d}$.

No. 3. - The Unsoundnesses and Defects of the Horse. Price 2 s. 6 d., or mounted, 5 s. 6 d.

No. 4. - The Shoeing of the Horse, Mule and Ox. Price 2 s. 6 d., or mounted, $5 \mathrm{~s}$. $6 \mathrm{~d}$.

No. 5.-The Elementary Anatomy, Points and Butcher's Joints of the Ox. Price 3s. 6d., or mounted, 6s. 6d.

Price per set of Five, 12s. ; or mounted, $27 \mathrm{~s}$. 
NOW READY. Price £6 nett.

\section{A NEW AND GREATLY IMPROVED EDITION}

or

\section{WHITE'S PHYSIOLOGICAL MANIKIN.}

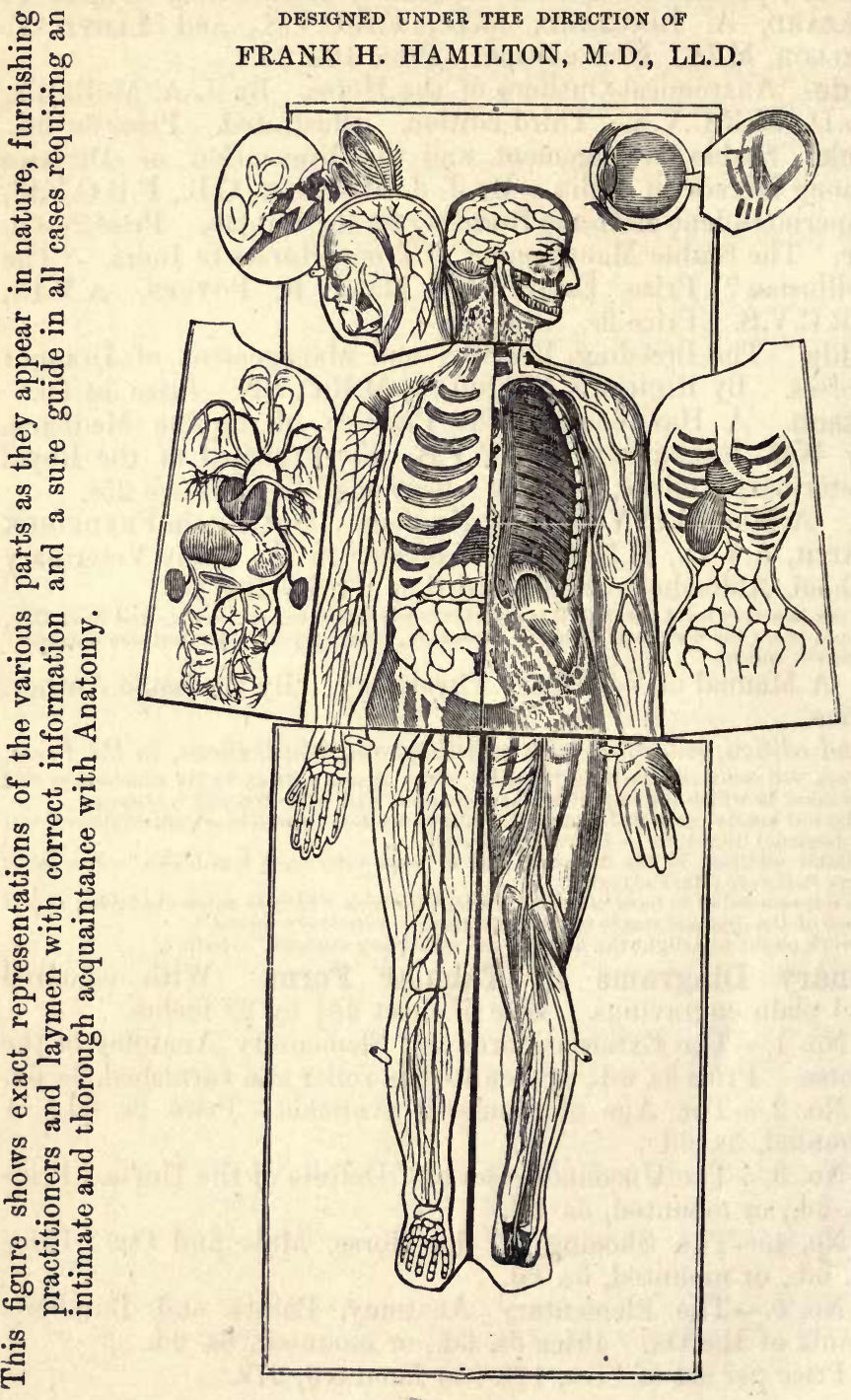

E

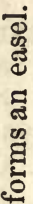

ฮีँ

这这

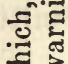

1.

๑ొ สี

ฮึ

ช్

है

홍

量

ณ

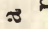

:

क्षे

용

웅

골

동

ช

(⿻)

三

.29 

RETURN TO the circulation desk of any University of California Library

or to the

NORTHERN REGIONAL LIBRARY FACILITY

Bldg. 400, Richmond Field Station

University of California

Richmond, CA 94804-4698

ALL BOOKS MAY BE RECALLED AFTER 7 DAYS

- 2-month loans may be renewed by calling (510) 642-6753

- 1-year loans may be recharged by bringing books to NRLF

- Renewals and recharges may be made 4 days prior to due date.

DUE AS STAMPED BELOW

\section{AUG 091996}



